\title{
Label-Free Measurements of Amyloid Formation by Suspended Microchannel Resonators
}

\author{
Dissertation \\ zur Erlangung des mathematisch-naturwissenschaftlichen Doktorgrades \\ "Doctor rerum naturalium" \\ der Georg-August-Universität Göttingen \\ im Promotionsprogramm ProPhys \\ der Georg-August University School of Science (GAUSS)
}

vorgelegt von

Yu Wang

aus Shanxi, V. R. China

Göttingen, 2013 
$\underline{\text { Betreuungsausschuss }}$

Dr. Thomas P. Burg, Biologische Mikro- and Nanotechnologie, Max-Planck-Institut für Biophysikalische Chemie

Prof. Dr. Christoph F. Schmidt, Molekulare und Zelluläre Biophysik, Universität Göttingen - Drittes Physikalisches Institut - Biophysik

Mitglieder der Prüfungskommission

Referent: Prof. Dr. Christoph F. Schmidt, Molekulare und Zelluläre Biophysik, Universität Göttingen - Drittes Physikalisches Institut - Biophysik

Korreferent: Dr. Thomas P. Burg, Biologische Mikro- and Nanotechnologie, Max-Planck-Institut für Biophysikalische Chemie

$\underline{\text { Weitere Mitglieder der Prüfungskommission: }}$

Prof. Dr. Markus Zweckstetter, Proteinstrukturbestimmung Mittels NMR, MaxPlanck-Institut für Biophysikalische Chemie

Prof. Dr. Sarah Köster, Nanoscale Imaging of Cellular Dynamics, Universität Göttingen - Institut für Röntgenphysik

Prof. Dr. Tiago Fleming Outeiro, Neurodegeneration und Neurorestaurationsforschung, Universitätsmedizin Göttingen

Prof. Dr. Peter Jomo Walla, Biomolekulare Spektroskopie und Einzelmoleküldetektion, Max-Planck-Institut für Biophysikalische Chemie

Tag der mündlichen Prüfung: 


\section{Abstract}

Amyloid deposition in tissues is a hallmark of many severe diseases including Alzheimer's and Parkinson's disease. The formation of amyloid fibrils involves several intermediate steps, and revealing the kinetics of each step is of great importance for a deeper understanding of the underlying mechanisms. However, conventional colorimetric or fluorescent assays, such as Thioflavin T, are not well suited for precise measurements of aggregation kinetics, as these dyes are only sensitive to specific states of the aggregation and their binding may interfere with the process. Biosensor techniques are superior in this regard since the measurement is label-free. However, established methods such as Surface Plasmon Resonance (SPR) and Quarz Crystal Microbalance (QCM) have several limitations, including large sample consumption, low sensitivity, and complex data analysis. This thesis presents, for the first time, the label-free kinetic measurement of amyloid formation by mass using Suspended Microchannel Resonators (SMR). SMR devices achieve low sample consumption at high mass resolution and provide a direct readout of the buoyant mass of aggregates.

As a prerequisite for quantitative measurements of amyloid elongation kinetics by SMR, a new method for the reliable immobilization of seed fibrils inside the device was first established. The method allowed the reproducible deposition of ensembles of insulin amyloid fibrils inside the $3 \mu \mathrm{m}$ tall detector channels without clogging. To this end, a robust vapor-based method for the deposition of aminosilane layers in closed microfluidic systems has been developed, and a novel quantitative fluorescence imaging method was introduced to optimize the density, quality and stability of these layers in situ.

In the surface-based SMR measurements, the reproducible seed fibril immobilization has been confirmed by having consistent surface fibril density of $734 \pm 89$ molecules $/ \mu \mathrm{m}^{2}$. After prevention of non-specific protein adsorption and validation of the stability, the fibril elongation rates and the absolute rate constants can be readily determined. For $1 \mathrm{mg} / \mathrm{mL}$ monomer concentration, the results show that the binding of a monomer to a fibril occurs every $4.9 \pm 0.5 \mathrm{~s}$, corresponding to the rate constant of $(1.2 \pm 0.1) \times 10^{3} \mathrm{M}^{-1} \mathrm{~s}^{-1}$ at $37^{\circ} \mathrm{C}$. Further on-rate 
measurements depending on monomer concentration and temperature provide insights into the mechanism and thermodynamics of the process. A two-step "docklock" elongation model fits well with the measurements. The determined enthalpy and the entropic contribution of the activation are in agreement with literature data and indicate in general an enthalpically unfavorable but entropically favorable process.

In the last part of the thesis, effects of shear flow and additives such as metal ion salts, natural products and small organic compounds, have been studied by measuring the fibril elongation rates. In contrast, dissociation experiments using the surface-based SMR technique have been applied to monitor the degradation of the immobilized fibrils. Both approaches are demonstrated to be promising platforms for screening potential inhibitors and therapeutic drugs for amyloid-related diseases. 


\section{Acknowledgements}

This thesis would not have been possible without the help and support of many people to whom I would like to express my sincere gratitude.

Firstly, I would like to deeply acknowledge the great support of my supervisor Dr. Thomas P. Burg, who opened the door for me to the fascinating world of microfluidics. I am extremely grateful to him for the opportunity he gave me to work on this exciting project, for always being available to answer my questions, and for his patience, supportive guidance and constructive discussions throughout the study. His passion and enthusiasm for exploring the fundamental of every scientific question have been a continuous source of encouragement for me and will continue to inspire me in future.

I also would like to thank Prof. Christoph F. Schmidt for accepting me as a PhD student at the Faculty of Biophysics at the University of Göttingen, for guiding me through the program and for his helpful advice during committee meetings. Furthermore, I would like to thank Prof. Markus Zweckstetter, Prof. Dr. Sarah Köster, Prof. Dr. Tiago Fleming Outeiro and Prof. Dr. Peter Jomo Walla for agreeing to be members of my $\mathrm{PhD}$ thesis committee. I am very grateful to Prof. Markus Zweckstetter for his help and for constructive advice relating to the amyloid fibril study as well as for providing samples and chemicals from his group.

I am greatly indebted to all former and current colleagues in our research group for the very pleasant and supportive working environment. I would like to thank Dr. Rachel Lowe for constructive discussions and for her support and encouragement in writing my first paper and for proof-reading this thesis. I would like to thank Dr. Yara Mejia for her valuable contribution to my first paper and I would also to acknowledge the constructive discussions everyone was involved in during group seminars. I would like to thank Mario Modena. He, with his strong background in physical engineering, provided great support with regard to instrumentation. I also wish to thank Nikunj Dudani for his outstanding work doing regeneration studies 
of transparent microchannels.

Furthermore, I would like to thank Dr. Iwan Schaap and his student Mitja Platen for assisting me to perform AFM experiments in their laboratory. Also, I would like to thank the staff who work in different facilities in the Max-Planck-Institute for Biophysical Chemistry. I would like to thank Dr. Dietmar Riedel and Gudrun Heim in the facility of electron microscopy for the EM analysis of my biological samples. I would like to thank Rainer Schürkötter and the staff in the workshop for precise manufacturing numerous small manifold parts for me. I would like to thank the facilities of IT \& Electronics Service and MediaService for their continuous support throughout the work.

I wish to deeply thank all my friends for their support and precious friendship over the years. Additionally, I would like to thank Dr. Han Sun for this $\mathrm{LTT}_{\mathrm{E} X-}$ template. I am also very grateful to Dr. Kaomei Guan-Schmidt and Jean-Philippe Demers for proof-reading the thesis.

Finally, I would like to express my sincere gratitude to my family - my husband, my son, my parents, my grandparents and all my family members for the unconditional love, understanding, trust and immeasurable support throughout my life. With you and for you, I had the ability and energy to be here and achieve this. 


\section{Contents}

Abstract $\quad$ V

$\begin{array}{lll}\text { Acknowledgements } & \text { VII }\end{array}$

$\begin{array}{ll}\text { Contents } & \text { IX }\end{array}$

$\begin{array}{ll}\text { List of Abbreviations } & \text { XIII }\end{array}$

$\begin{array}{lll}\text { Figures } & \text { XVII }\end{array}$

$\begin{array}{ll}\text { Tables } & \text { XVIII }\end{array}$

1 Introduction 1

1.1 Protein Aggregation and Amyloid Fibrils . . . . . . . . . . . . . . . . 1

1.1.1 Structure, Characteristics and Properties . . . . . . . . . . 3

1.1.2 Kinetics, Mechanisms and Energy Landscape . . . . . . . . . 5

1.1.3 Diagnosis and Inhibition of Neurodegenerative Diseases . . . . 10

1.2 Detection Techniques . . . . . . . . . . . . . . . . . . . . . . . . . . . . . . . . . . 13

1.3 Motivation . . . . . . . . . . . . . . . . . . 18

2 Theory 23

2.1 SMR . . . . . . . . . . . . . . . . . 23

2.1.1 Suspended Microchannel Resonators (SMR) . . . . . . . . . 24

2.2 Protein Immobilization . . . . . . . . . . . . . . . . . 30

2.2.1 Covalent Coupling . . . . . . . . . . . . . 31

2.2.2 Physisorption . . . . . . . . . . . . . . . . 35

2.2 .3 Bioaffinity Interaction . . . . . . . . . . . . . 37

2.2.4 Combination of Immobilization Techniques . . . . . . . . . . . 37

2.3 Surface Passivation . . . . . . . . . . . . . . . . . . . . 41

3 Materials and Methods $\quad 43$

3.1 Chemicals and Materials . . . . . . . . . . . . . . . . 43

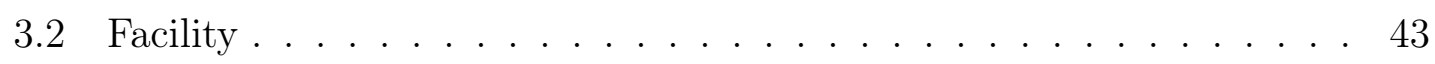


3.3 Sample Preparation . . . . . . . . . . . . . . . . . . . . . . . . 46

3.3.1 Fabrication of SMR Devices . . . . . . . . . . . . 46

3.3.2 Fabrication of Transparent Microchannels . . . . . . . . . 46

3.3 .3 Preparation of Insulin Fibrils _. . . . . . . . . . . . . 46

3.3 .4 Preparation of $\alpha$ S Fibrils . . . . . . . . . . . . . . . 48

3.3.5 Surface Functionalization with Silane Coupling Agents . . . . 48

3.3.6 Protein Immobilization and Passivation . . . . . . . . . . . . 49

3.3.6.1 Covalent Coupling of Insulin Fibrils . . . . . . . . . . 49

3.3.6.2 Electrostatic Adsorption of $\alpha$ Synuclein . . . . . . . 51

3.3.7 Microchannel Surface Regeneration . . . . . . . . . . . . . 52

3.4 Biophysical Detection Techniques _ . . . . . . . . . . . . . 52

3.4.1 Fluorescence Quantification of Amine Groups . . . . . . . 52

3.4.1.1 Fluorescence Labeling . . . . . . . . . . . . . 52

3.4.1.2 Fluorescence Imaging and Intensity Measurements . 53

3.4.1.3 In situ Stability Measurements . . . . . . . . . . . 54

3.4.2 Fluorescence Imaging of Immobilized Proteins . . . . . . . . . 55

3.4.3 SMR Kinetic Measurements . . . . . . . . . . . . . . 55

3.4.4 Fluorospectrometer Measurements . . . . . . . . . . . 57

3.4.5 UV-VIS Spectrometer Measurements . . . . . . . . . . . 58

3.4.6 X-ray Photoelectron Spectroscopy Analysis (XPS) . . . . . . 58

3.4 .7 Electron Microscopy . . . . . . . . . . . . . . . . . . 59

3.4 .8 Atomic Force Microscopy . . . . . . . . . . . . . . . . . . . 59

4 SMR Surface Modification and Characterization by Fluorescence Microscopy 61

4.1 Introduction . . . . . . . . . . . . . . . . 61

4.2 Electrostatic Immobilization . . . . . . . . . . . . . . . 63

4.3 Covalent Immobilization . . . . . . . . . . . . . . . . 66

4.3.1 In-Channel Surface Functionalization . . . . . . . . . . . 66

4.3.1.1 Vapor-Based Surface Functionalization with Aminosilanes . . . . . . . . . . . . . . 66

4.3.1.2 Surface Quantification of Aminosilane Functionalization .................. 66

4.3.1.3 Quantification of Vapor-Deposited APTMS and APDMMS Layers . . . . . . . . . . . . . . 72

4.3.1.4 In situ Stability Monitoring of Aminosilane Functionalization . . . . . . . . . . . . . 73

4.3.1.5 Protein Binding Capacity of Amine-Functionalized Surfaces Using Glutaraldehyde Coupling . . . . . . . 77 
4.3.1.6 Surface Functionalization with Carboxymethyl Dextrans . . . . . . . . . . . . . 79

4.3.2 Immobilization of Insulin Fibrils . . . . . . . . . . . . . . 81

5 Kinetic Measurements of Amyloid Fibril Growth using SMR $\quad 87$

5.1 Introduction . . . . . . . . . . . . . . . . . . . 87

5.1.1 Sensitivity of Surface-Based SMR Measurements . . . . . . . . 88

5.1.2 Comparison of Reproducibility and Stability Using Electrostatic and Covalent Immobilization Techniques . . . . . . . . . 89

5.2 Elongation Kinetics . . . . . . . . . . . . . . . . . . . . . . 94

5.2.1 Surface Density of Immobilized Insulin Seed Fibrils . . . . . . 94

5.2.2 Determination of Absolute Rates of Insulin Fibril Elongation by SMR . . . . . . . . . . . . . . . . . . . . 95

5.3 Environmental Effects on Kinetics . . . . . . . . . . . . . . . . 98

5.3.1 Insulin Elongation Kinetics as a Function of Temperature and Monomer Concentration . . . . . . . . . . . . . . . 99

5.3.2 Shear Effect on Insulin Elongation Kinetics . . . . . . . . . . . 104

5.3.3 Influence of Additives on Elongation Kinetics . . . . . . . . . 107

5.3.3.1 Kinetics with Additional Salt and Metal Ions . . . . 107

5.3.3.2 Kinetics with Additional Natural Products . . . . . . 108

5.3.3.3 Kinetics with Small Aromatic Organic Compounds . 109

5.4 Fibril Dissociation . . . . . . . . . . . . . . 111

6 Conclusions

$\begin{array}{lr}\text { Bibliography } & 119\end{array}$

$\begin{array}{lr}\text { Appendix } & 147\end{array}$

$\begin{array}{ll}\text { Lebenslauf } & 151\end{array}$

$\begin{array}{lc}\text { Publications } & 153\end{array}$ 


\section{List of Abbreviations}

$\begin{array}{ll}\text { AA } & \text { Amino acids } \\ \text { AF555-NHS- } & \text { Alexa Fluor }{ }^{\circledR} \text { 555 carboxylic acid, succinimidyl ester } \\ \text { ester } & \\ \text { AFM } & \text { Atomic Force Microscopy } \\ \alpha \text { S } & \alpha \text {-Synuclein } \\ \text { APDMMS } & \text { 3-Aminopropyl dimethylmethoxysilane } \\ \text { APTES } & \text { 3-Aminopropyl triethyoxysilane } \\ \text { APTMS } & \text { 3-Aminopropyl trimethoxylsilane } \\ \text { BMH } & \text { Bis(maleimido)hexane } \\ \text { BSA } & \text { Bovine serum albumin } \\ \text { CD } & \text { Circular Dichroism Spectroscopy } \\ \text { CDI } & \text { Carbodiimid } \\ \text { CM-dextran } & \text { Carboxymethyl-dextran } \\ \text { CMP } & \text { Chemical Mechanical Polishing } \\ \text { DSS } & \text { Disuccinimidyl suberate } \\ \text { DTT } & \text { Dithiothreitol } \\ \text { DRIE } & \text { Deep Reactive Ion Etching } \\ \text { EDC } & \text { N'-(3-dimethylaminopropyl)-3-ethyl carbodiimide hydro- } \\ & \text { chloride } \\ \text { EM } & \text { Electron Microscopy } \\ \text { EMCS } & \text { N-( } 6 \text {-maleimidocaproyl)succinimide } \\ \text { EPPTMS } & \text { 3-(2,3-epoxypropoxy)-propyl)-trimethoxysilane } \\ \text { HEPES } & \text { 4-(2-Hydroxyethyl)-1-piperazineethanesulfonic acid } \\ \text { FEP } & \text { Fluorinated ethylene propylene } \\ \text { GA } & \text { Glutaraldehyde } \\ \text { GFP } & \text { Green Fluorescence Proteins } \\ \text { GOPTES } & \text { 3-Glycidoxypropyl triethoxysilane } \\ \text { GOPTMS } & \text { I-Glycidoxypropyl trimethoxysilane } \\ \text { ICPTES } & \end{array}$


LPCVD Low-Pressure Chemical Vapor Deposition

MDS

Mercapto-methyldimethylethoxysilane

MTS

3-Mercaptopropyltrimethoxysilane

NDA

Naphthalene-2,3-dicarboxaldehyde

NHS

N-hydroxysuccinimide

NMR

Nuclear Magnetic Resonance

OWS

Wavelength Interrogated Optical Sensors

PBS

Sodium phosphate buffer

PEG

Polyethyleneglycol

PLGA

Poly-L-glutamic acid

PLL

Poly-L-Lysine

$\mathrm{QCM}$

Quarz Crystal Microbalance

QLS

Quasielastic Light Scattering

RIE

Reactive Ion Etching

SEM

Scanning Electron Microscopy

SMR

Suspended Microchannel Resonators

SPDP

N-succinimidyl-3-(2-pyridyldithio) propionate

SPR

Surface Plasmon Resonance

S-SMCC

Sulfo-succinimidyl-4-(p-maleimidophenyl)butyrate

S-SMPB

Sulfo-succinimidyl-4-(N-maleidomethyl)-cyclohexane-1-

carboxylate

Sulfo-NHS-LC- $\quad$ EZ-link ${ }^{\mathbb{B}}$ sulfosuccinimidyl 6-(biotinamido) hexanoate

biotin

TCEP

Tris(2-carboxyethyl)phosphine

TEM

Transmission Electron Microscopy

TIRFM

Total Internal Reflection Fluorescence Microscopy

TLM

Thermal Lens Microscopy

TMS-EDTA N-(Trimethoxysilylpropyl)ethylene-diamine triacetic acid, trisodium salt

TNB 5-Thio-2-nitrobenzoic acid

XPS

X-ray Photoelectron Spectroscopy 


\section{List of Figures}

1.1 Amyloid formation from diverse proteins . . . . . . . . . . . . . . 3

1.2 Amyloid $\beta$-Strand and the X-ray diffraction pattern . . . . . . . . . . 4

1.3 Nucleation-dependent polymerization model . . . . . . . . . . . . 6

1.4 Formation pathways of lysozyme amyloid. . . . . . . . . . . . . 7

1.5 A model of dock-lock mechanism based on SPR measurement . . . . 8

1.6 Energy landscape of protein folding and aggregation . . . . . . . . . . 9

1.7 Strategies to inhibit amyloid formation . . . . . . . . . . . . . . . 11

1.8 Working principles of TIRFM, AFM, QCM and SPR to study amyloid elongation . . . . . . . . . . . . . . . . . . 18

1.9 Working principle of SMR detection for amyloid elongation. . . . . . 19

2.1 Schematics of SMR devices . . . . . . . . . . . . . . . . . . 25

2.2 Two detection modes of SMR measurements . . . . . . . . . . . . 26

2.3 A double clamped beam to illustrate the mass sensitivity of an SMR device . . . . . . . . . . . . . . . . . . 27

2.4 Schematic drawing of protein immobilization with different binding geometries . . . . . . . . . . . . . . . . 31

2.5 Protein immobilization concepts via covalent coupling, phsisorption or bioaffinity interaction . . . . . . . . . . . . . . 31

2.6 Covalent immobilization of proteins to silicon and glass surfaces with different functional groups . . . . . . . . . . . . . . . . . 39

2.7 Mechanisms of covalent coupling of alkoxysilanes with surface hydroxyl groups . . . . . . . . . . . . . . . . . . . . . . . . . . 4 40

3.1 Fabrication and packaging process of nanochannels . . . . . . . . 47

3.2 Reaction schematic for covalent labeling of microchannel surfaces with NHS-based molecules . . . . . . . . . . . . . . . . . . . 49

3.3 Covalent protein immobilization using Glutaraldehyde . . . . . . . . . 51

3.4 Fluorogenic reaction using NDA to label amine groups on the surfaces 54

3.5 Experimental setup for the surface-based SMR measurements . . . . 56

4.1 Microfluidic devices for fluorescence microscopy detection . . . . . . . 62

4.2 Electron micrographs of $\alpha \mathrm{S}$ monomers and fibrils . . . . . . . . . . 64 
4.3 Fluorescence images showing $\alpha$ S-fibril immobilization on microchannel surfaces . . . . . . . . . . . . . . . . . . 6 65

4.4 ThT binding to amyloid fibrils . . . . . . . . . . . . . . 66

4.5 Surface modification with APTMS . . . . . . . . . . . . 67

4.6 Fluorescence detection of functional surfaces labeled with fluorophors 68

4.7 Linear correlation between fluorescence and surface coverage . . . . . 70

4.8 Comparison of surface coverage of amine groups using APTMS and APDMMS ....................... 73

4.9 XPS survey spectra of modified silicon substrates stability in solutions. 74

4.10 Stability measurements of APTMS modified surface . . . . . . . . . . 75

4.11 Regeneration of amine functional groups on surfaces . . . . . . . . . . 76

4.12 Schematic showing possible monomeric and polymeric structures of glutaraldehyde. . . . . . . . . . . . . . . . . . 78

4.13 Fluorescence images of immobilized GFP . . . . . . . . . . . . . . . 79

4.14 Stability test of adsorbed GFP on microchannel surfaces . . . . . . 80

4.15 Schematics for immobilization of GFP proteins to the carboxymethyldextran hydrogels on microchannel surfaces . . . . . . . . . . . 81

4.16 Fluorescence images of immobilized GFP proteins in APTMSmodified microchannels using different CM-dextran coupling methods. 82

4.17 Insulin structure with two polypeptide chains. . . . . . . . . . . . 82

4.18 Electron micrographs of insulin monomers and fibrils . . . . . . . . . 84

4.19 Fluorescence image of immobilized insulin fibrils inside microchannels 84

5.1 Calibration of the SMR device . . . . . . . . . . . . . . . 89

5.2 SMR measurements of binding of $\alpha \mathrm{S}$ monomers and fibrils to PLLcoated surfaces . . . . . . . . . . . . . . . . . . . . . 91

5.3 Comparison of reproducibility of fibril elongation measurements by SMR using different immobilization techniques . . . . . . . . . . . . 93

5.4 Stability test of covalently immobilized insulin fibrils . . . . . . . . . 94

5.5 Immobilization of insulin seed fibrils on SMR microchannel surfaces. . 95

5.6 AFM images of APTMS-modified Si-substrates . . . . . . . . . . 96

5.7 Seed fibril growth with $1 \mathrm{mg} / \mathrm{mL}$ monomer solution detected by SMR 97

5.8 SMR measurements of seed fibril elongation . . . . . . . . . . . . . . 98

5.9 Solubility test of insulin monomers at various $\mathrm{pH}$ values detected by

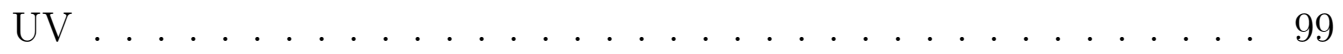

5.10 Kinetic measurements of seed fibrils by SMR at various concentrations and temperature . . . . . . . . . . . . . . . 101

5.11 Schematic representation of the dock-lock mechanism based on SMR detection . . . . . . . . . . . . . . . . . . . 101 
5.12 Arrhenius plot for insulin elongation processes . . . . . . . . . . 103

5.13 Correlation of transit time and pressure drop in SMR microchannels . 105

5.14 Test of elongation rates with additives of metal ions $\left(\mathrm{NaCl}, \mathrm{ZnCl}_{2}\right.$,

$\mathrm{MgCl}_{2}, \mathrm{CaCl}_{2}$ ) . . . . . . . . . . . . . . 109

5.15 Test of elongation rates with additives of ectoine and trehalose . . . 110

5.16 Test of elongation rates with additives of PcTS and methylene blue . 111

5.17 Dissociation monitoring of insulin fibrils . . . . . . . . . . . . 113 


\section{List of Tables}

1.1 Amyloid-related diseases and precursors . . . . . . . . . . . . . 2

1.2 Inhibitors reported for neurodegenerative diseases. . . . . . . . . . . . 12

1.3 Techniques applied for amyloid formation. . . . . . . . . . . . . . 15

2.1 Surface functionalization of silicon/glass-based microfluidic devices for protein immobilization. . . . . . . . . . . . . . . 33

3.1 Chemicals for the experiments. . . . . . . . . . . . . . . . 44

3.2 Important facilities used in the experiment. . . . . . . . . . . . . . 45

4.1 XPS validation of amine functionalization . . . . . . . . . . 75

5.1 Fitting parameters for the dock-lock mechanism . . . . . . . . . . . 102

5.2 Thermodynamic parameters for amyloid-related proteins. . . . . . . . 104

6.1 Fitting parameters for time-dependent fluorescence detection ${ }^{[1]}$. . . . 148

6.2 Standard statistical errors for fitting parameters ${ }^{[1]} \ldots$. . . . . . . . . . 149 


\section{Chapter 1}

\section{Introduction}

Amyloid formation is the hallmark of over 40 human diseases and arises from abnormal protein aggregation [65]. Novel techniques are urgently required to comprehensively understand the kinetics, mechanisms and energy landscape of the process. This chapter focuses first on the biological background (the significance, properties, mechanism hypothesis and energy aspect) of protein aggregation and amyloid fibrils in Section 1.1. In Section 1.2, detection techniques, in particular the surface-based methods to study amyloid fibril formation, are reviewed, and the significance of developing novel techniques is discussed to address current technical limitations. At the end of this chapter (Section 1.3), the motivation and aim of this thesis are presented.

\subsection{Protein Aggregation and Amyloid Fibrils}

Protein aggregation is a ubiquitous process of molecular self-assembly in biology [267]. Abnormal protein misfolding may lead to amyloid formation. Tissue deposits of such highly fibrous structured protein aggregates have been reported to be a pathological feature of a large number of human and animal diseases as shown in Table 1.1 [23,65,86,102,103,137,139, 176,211,220,233,239, 245]. These include two of the most common neurodegenerative disorders in the aging population - Alzheimer's and Parkinson's disease, and other diseases caused by local or systemic amyloidosis such as localized amyloidosis at the place of repeated insulin injection. The prevalence of such diseases is still steadily increasing and is expected to double by the middle of the century [27,229].

Amyloid fibrils have been recognized as the pathologic signs since the early 1850s, and major advances in understanding amyloid formation as the key feature 
Table 1.1: Amyloid fibril related human and animal diseases and their precursors [102, $205,232,239,264,276$

\begin{tabular}{lll}
\hline \hline & Disease & Precursor \\
\hline Neurodegenerative & Alzheimer's disease(AD) & A $\beta(1-42)$, Tau \\
diseases & Parkinson's disease (PD) & $\alpha$-synuclein \\
& Prion diseases (e.g. TSE) & Prion \\
& Huntington's disease (HD) & Huntingtin \\
& Serpinopathies & Neuroserpin \\
& Progressive supranuclear palsy & Tau \\
& (PSP) & Heat shock proteins \\
Systemic & Spinocerebellar ataxias (SCA) & Tau, Ataxins \\
amyloidosis & Diabetes type II & Amylin \\
& Haemodialysis related A & $\beta$-2 Microglobulin \\
Localized & Cystic fibrosis & CFTR protein \\
amyloidosis & Injection Amyloidosis & Insulin \\
& Aging pituitary & Prolactin \\
Animal amyloidosis & Conea & Lactoferrin \\
& Age-related disease (Dog) & Apolipoprotein AI \\
& Plasmocytoma (Cat, Horse) & Immunoglobulin \\
\hline \hline
\end{tabular}

of many diseases have been achieved in the last 40-50 years, [95, 136,233]. However, the complicated processes of amyloid formation are still poorly understood so far. Diverse proteins of different size, function and sequence polymerize into unbranched fibrils with similar ultrastructural properties and appearance (Figure 1.1) $[23,195,245]$.

Recent studies show that amyloid fibrils can be obtained in vitro from proteins that are exposed to extreme conditions, e.g. low $\mathrm{pH}$, chemical or heat denaturation $[4,23,24,41,65,87]$. This leads to the conclusion that amyloid fibrils are possibly a common state of proteins and their formation limits the production and use of proteins in a wide range of pharmaceutical and biotechnological applications. Conversely, experiments to study amyloid formation can be carried out in vitro and may lead to more comprehensive understanding of the process. In the following sections, previous studies and hypothesis on amyloid structure and formation mechanism will be reviewed and the reported development of therapies for neurodegenerative diseases are summarized. 


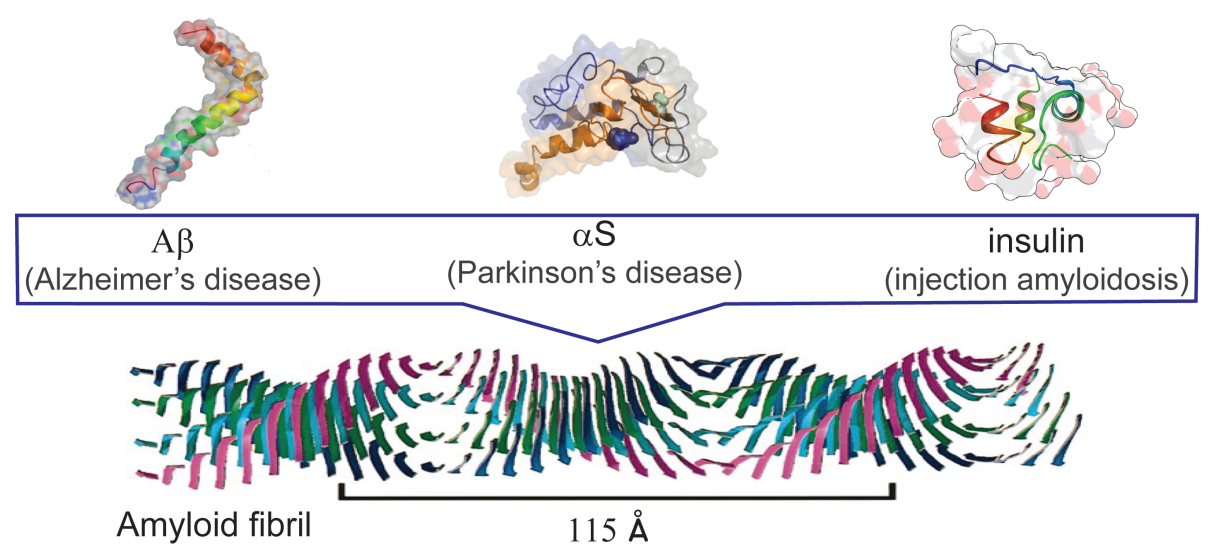

Figure 1.1: Amyloid fibril formation from diverse proteins. Images of proteins and fibrils are adapted from Protein Data Bank (PDB) and the reference [245].

\subsubsection{Structure, Characteristics and Properties}

The structural understanding of amyloids has advanced with available technologies. Divry and Florkin in the 1920s first reported the tinctorial property of amyloid fibrils by showing the apple green birefringence of the fibrils when stained with Congo Red dyes and observed under polarized light [195]. Because the Congo Red staining assay has later been proved to show false positive response in some cases $[131,155]$, the fluorescent property with Thioflavin $\mathrm{T}$ (ThT) assay has been widely applied to indicate the presence of amyloid formation in vitro (Section 1.2).

The ultrastructure of amyloid fibrils was first defined crystallographically with X-ray diffraction studies in the late 1960 s $[38,70,87,245]$. The X-ray pattern shows that one of the principle features of amyloids is pleated cross- $\beta$ sheets with ca. $4.7 \AA$ inter-chain spacing and ca. $10-11 \AA$ inter-strand spacing, as shown in Figure 1.2. The $\beta$-sheet strands are aligned perpendicular to the long axis of the fibrils. Circular dichroism spectroscopy (CD), cryo-electron microscopy [120] and nuclear magnetic resonance (NMR) [78, 213, 243] also confirm the existence of the $\beta$-sheet structure in mature amyloid fibrils and protofibrils. As depicted in Figure 1.2, $\beta$-strands of LVEALYL peptide have been suggested to be the main contributor to the spine formation of insulin fibrils [116]. The pair structure shows the packaged atomic structure as a result of steric zipper interaction. The steric zipper interface is typical for amyloid formation, since similar structures have been reported with several other amyloid fibrils [102,183,243]. Most $\beta$-sheet structures of amyloid fibrils have a right-handed twist of $0-30^{\circ}$ between neighbouring $\beta$-strands. The twisting around a common helical axis accounts for the repeating unit of ca. $115 \AA$ (1.1) [245]. It has been demonstrated that this helical structure enables 
formation of hydrogen bonding between the $\beta$-strands and thereby enhances the rigidity and stability of amyloids [184]. Some studies have shown that amyloid fibrils are even highly resistant to proteases [193,228]. Electron micrographs show that all amyloid fibrils consist of individual protofibrils of 40-100 $\AA$ in width and indeterminate in length, which may aggregate laterally to mature fibrils of up to $300 \AA$ in width [70,76,233, 256].

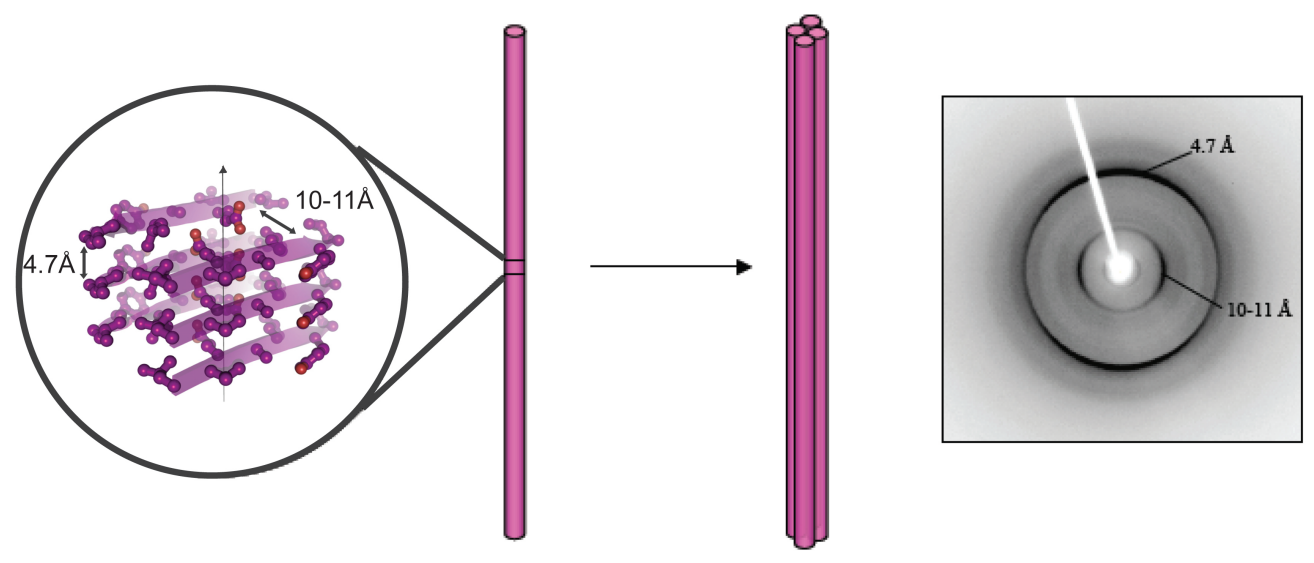

Figure 1.2: Schematics of $\beta$-strand pair formed by LVEALYL peptide, the association of protofibrils to mature fibrils and a classical X-ray diffraction pattern of cross- $\beta$ spine with 4.7-4.8 $\AA$ meridional and $10-11 \AA$ equatorial reflections. The view of the peptide $\beta$-strand pair as a result of steric zipper interaction is shown perpendicular to the fibril axis. The figure is adapted and partially modified from the references [168] and [116].

In order to elucidate basic properties of amyloid fibrils such as compactness, fluctuation, cavities and hydration, Akasaka et al. performed studies using a density meter and an ultrasonic velocity meter combined with CD on a solution of amyloid fibrils of hen lysozyme [3]. They concluded that the transition from a monomer into a protofibril can result in a large increase in partial specific volume of ca. $570 \mathrm{~mL} / \mathrm{mol}$ and in partial specific adiabatic compressibility coefficient of $8.83 \times 10^{-12} \mathrm{~cm}^{2} / \mathrm{dyn}^{-1}$. The high voluminous and compressible properties imply a cavity-rich, fluctuating nature of the amyloid protofibrils, which is in line with the above described $\beta$-strand spacing of the fibrils.

Mechanical properties of individual amyloid fibrils have also been studied with insulin fibrils as a model system using atomic force microscopy (AFM) [137, 235]. The strength of the fibrils has been characterized to be $0.6 \pm 0.4 \mathrm{GPa}$, comparable to that of steel $(0.6-1.8 \mathrm{GPa})$; and the mechanical stiffness has been measured to be $3.3 \pm 0.4 \mathrm{GPa}$ by AFM, comparable to that of silk (1-10 GPa). Interestingly, it has been discovered in nature that many insect silks, such as chrysopa fibres and spider silk, give a fibrous, cross-beta diagram in their natural state [87, 137,225,235]. 


\subsubsection{Kinetics, Mechanisms and Energy Landscape}

It has been widely accepted that amyloid fibril formation consists of many intermediate steps, yet the underlying mechanisms are still poorly understood. Comprehensive kinetic studies can provide opportunities to establish mechanistic models for the amyloid formation process. However, precise evaluation of the growth rates is challenging in bulk solution due to the complexity of many intermediate states involved and due to limitations of current detection techniques. In general, it is very challenging to establish models or rate equations to represent the nonlinear complex nature of the amyloid formation process. Progress, however, has been made in the development of detection techniques over the last 30-40 years.

Eaton, Ferrone, Hofrichter and coworkers introduced in the 1980s that heterogenous nucleation and filament fragmentation do occur during the hemoglobin S aggregation. Kinetic measurements using the fluorometric ThT method in solution also suggest a nucleation-dependent polymerization as depicted in Figure $1.3[136,239]$. In the suggested description, there is a slow lag phase during the transition from monomers to structured aggregates (nulei). This is a critical rate-limiting step for amyloid formation [195]. Once the nuclei are formed, they elongate rapidly to form larger protofibrils and fibrils. The lag phase can be eliminated by addition of pre-formed nuclei or short fibrils to protofibrils. This process is known as the seeding effect $[54,66,134]$. Furthermore, amyloid protofibrils can assemble into mature fibrils by fibril-fibril association.

Based on kinetic studies, a number of models for amyloid formation have been proposed, as reviewed by Zerovnik et al. and Kelly et al. [127,276]. Some popular models are listed below.

Template assisted (TA) model. In a rapid pre-equilibrium step, a soluble peptide in a random coil loosely binds to a pre-assembled nucleus. Then a rate determining structural change occurs to stabilize the peptide to fibril end.

Nucleated polymerization (NP) model. The rate-limiting step is the formation of a nucleus from an equilibrium including monomers that are or are not able to assemble. Once a nucleus is established, rapid assembly occurs by adding assembly competent monomers to the nucleus. 


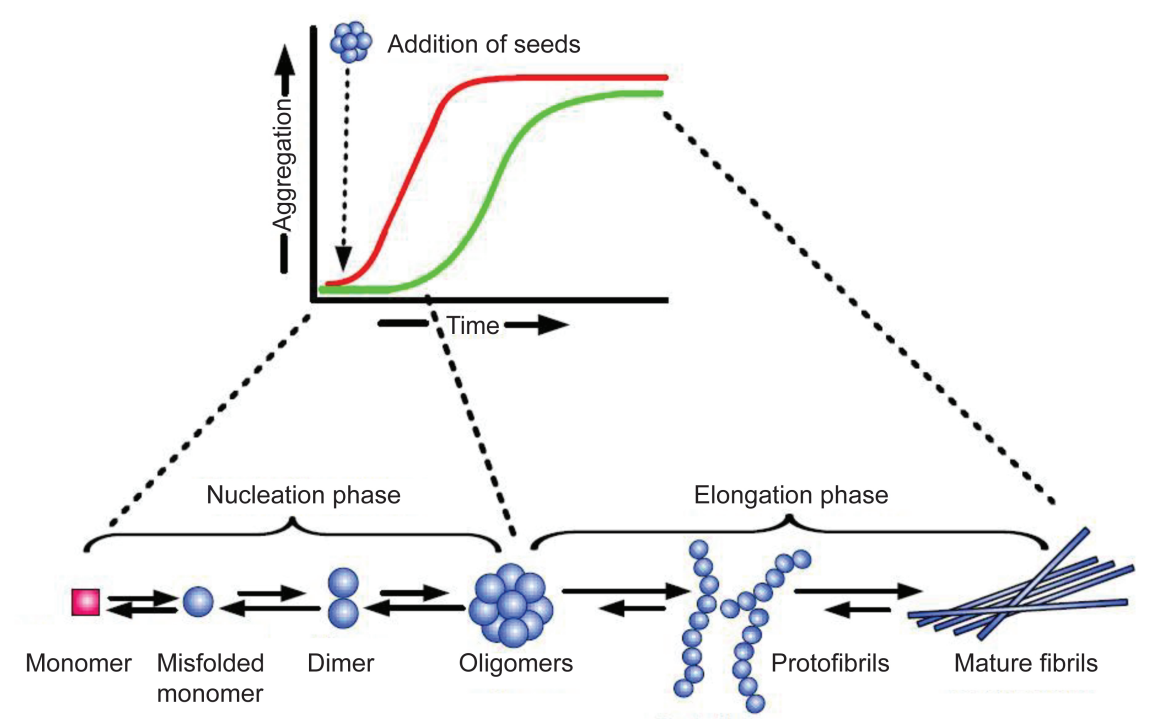

Figure 1.3: Nucleation-dependent polymerization model of amyloid aggregation, taken from Kumar et al. [143].

Monomer-derived conversion (MDC) model. In the rate-determining step, a monomer with a conformation in fibril-like state can associate with a monomer in the native state to form a dimer in fibril-analog state. After the dimer dissociation, both monomers in fibril-analog state rapidly bind to the ends of growing fibrils.

Nucleated conformational conversion (NCC) model. Monomers first accumulate to oligomers without conformational conversion. Association of the oligomers into nuclei with conformational conversion then takes place as the rate-determining step.

Off-pathway folding (OFF) model. In a parallel process to the on-pathway, by which structured oligomers are formed, irreversible amorphous intermediates and their domain-swapped dimers can be partially formed. Protofibril formation then takes place by end-to-end elongation of the intermediates. The protofibrils undergo further lateral and end-to-end association to form fibils.

It is difficult to judge which of the models best describes the general process of amyloid formation. It is also possible that several mechanisms fit various specific cases. Experimental evidence has also been reported to show diverse pathways in individual amyloid formation processes with different proteins $[45,66,86,90,96,118,127]$. A more comprehensive schematic including the possible pathways has been illustrated by Jahn and coworkers in Figure 1.4. Here, structured oligomeric nuclei can be formed either via partially unfolding of intermediates or via partial structuring of native or disordered aggregates. In 
addition, different amyloid-forming proteins sometimes show diversity with regard to oligomer formation following diverse possible mechanisms including domain swapping (ds), edge-edge-association (ee), strand association (sa) or $\beta$-strand stacking (bs) [66, 102], but they all convert into fibril seeds with the regular cross- $\beta$ secondary structure. Finally, similar as presented in Figure 1.3, the transition from oligomeric species to protofibrils and to further mature fibrils takes place through lateral association accompanied by some degree of structural reorganization [98]. In equilibrium, pathways such as monomer-dependent primary nucleation, filament fragmentation as secondary nucleation and secondary nucleation pathways are suggested to be responsible for the increase in the number of the nulei $[53,143]$.
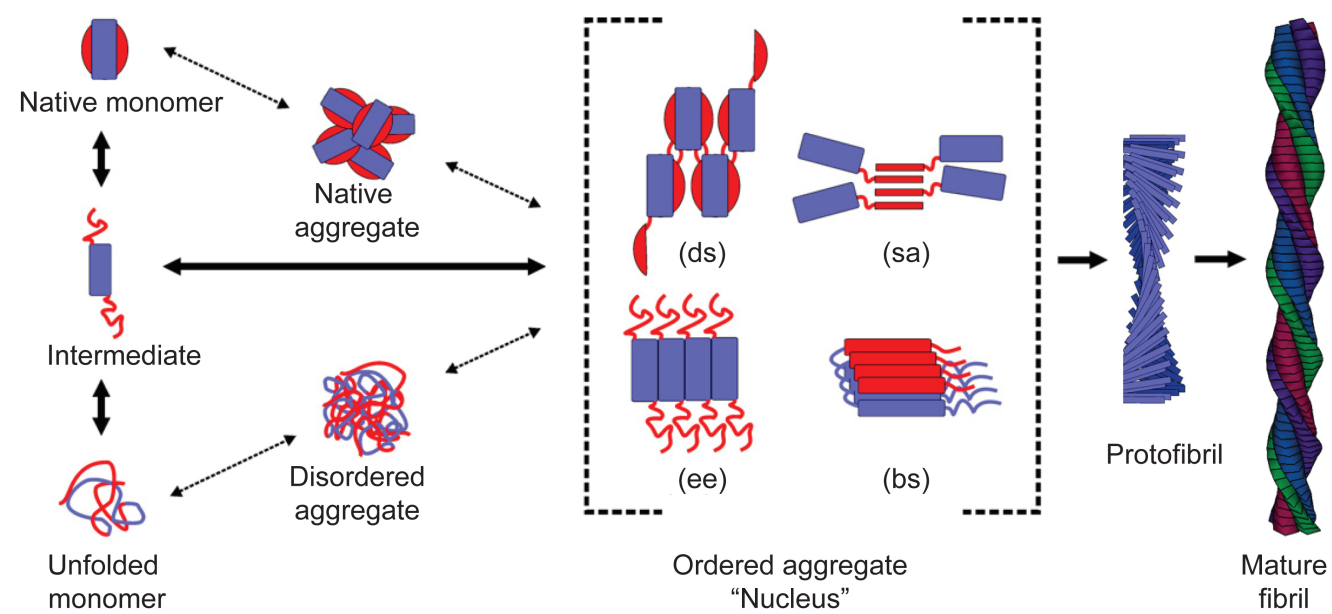

Figure 1.4: Amyloid fibril pathways in the case of lysozyme formation. Modified images adapted from Jahn et al. [90,118].

So far, the most significant achievement in kinetic measurements of the amyloid formation has been made in elongation processes, by which the sizes of existing aggregates increase rapidly. Early kinetic studies revealed a first-order dependence on the monomer and fibril concentrations, suggesting a bimolecular mechanism of growth by monomer attaching to the ends of fibrils. Recent kinetic measurements indicate a dock-lock-mechanism to be a general elongation process of amyloid fibrils $[39,74,92,138,169]$. The dock-step is described as attachment of monomers to fibril ends, while the lock-step is described as irreversible conversion of monomers to fibril ends due to conformational rearrangement. Despite the agreement with the dock-lock-mechanism as a general model for amyloid elongation, differences exist in the detailed steps based on the experimental data measured with individual methods. Esler et al. and Cannon et al. both measured the elongation of $\mathrm{A} \beta$ fibrils $[39,74]$. Using radioactive labeling of $\mathrm{A} \beta$ fibrils immobilized in 96 well plates, the measurements by Esler [74] suggested a two-step reaction model as depicted 
in Figure 1.5. The model includes a reversible dock process with dissociation of ca. 10 minutes half-life and an irreversible lock phase with dissociation of ca. 1000 minutes. In contrast, Cannon and coworkers found that a two-step model cannot sufficiently fit their kinetic data obtained by surface plasmon resonance (SPR) [39]. Instead, they proposed the results for a three-step model, by which an additional maturation step was introduced after the attachment step (dock) and the conformational rearrangement step (lock). Again, it is hard to judge which of both models best describes the actual process.

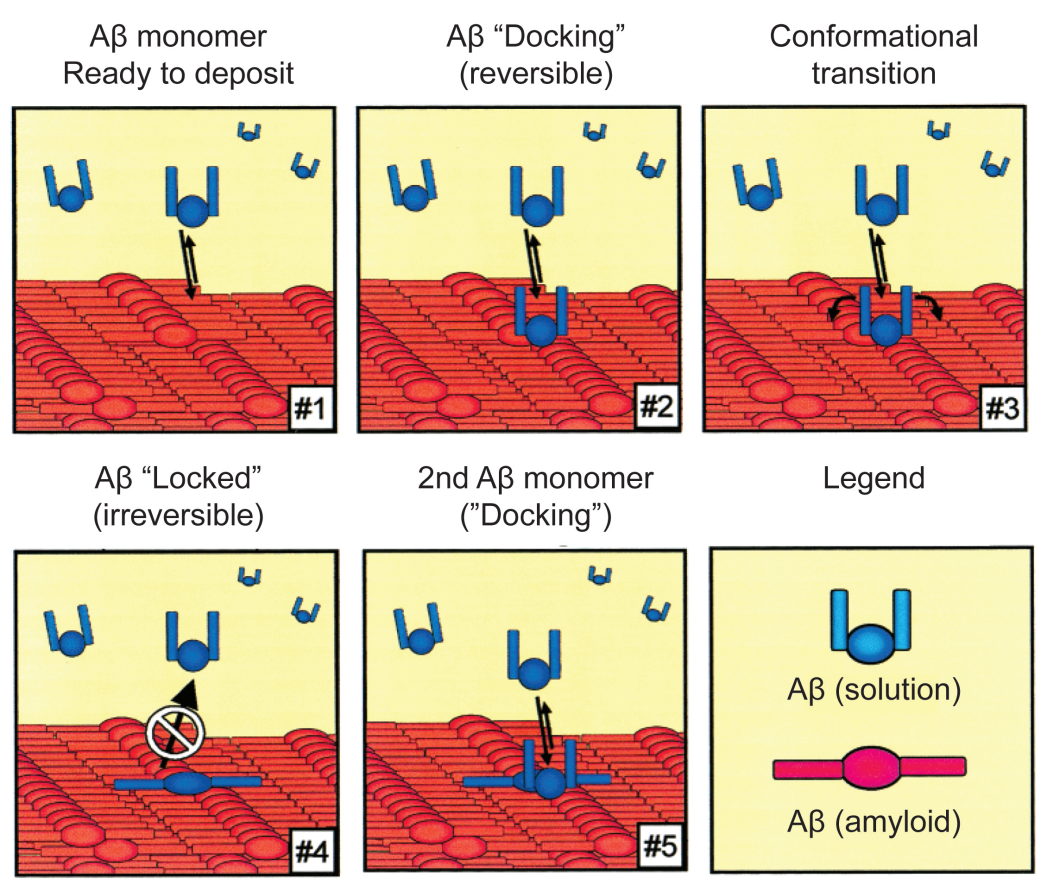

Figure 1.5: A model of dock-lock mechanism representing amyloid elongation in biosensor-based systems, taken from Esler et al. [74].

The dissociation processes lead to aggregate degradation or fragmentation, and therefore play significant role in molecular recycling $[41,53]$. In general, fibril dissociation is not usually observed, since the process is much slower than the aggregation growth that actually occurs. Due to the potential significance of amyloid dissociation for drug development, measurements of dissociation kinetics under different conditions were conducted as part of this thesis.

Amyloid formation has recently been interpreted as a consequence of changes in free-energy $[14,31,45,52,118,169,172]$, as graphically depicted in Figure $1.6[104,252]$. The $\mathrm{y}$-axis is a measure of the free energy and the $\mathrm{x}$-axis is a coordinate of various molecule conformations that successively exist during amyloid formation. The funnel-shaped energy landscape indicates the preferred 
conformations under given conditions by following the "down-hill" path. Generally, the nucleus formation from native monomers to oligomers is thermodynamically unfavourable. Native and metastable non-native intermediates are possibly present during the folding process due to the ruggedness of the landscape. Compared to the unfolded state, the partially folded states or misfolded states are both thermodynamically and kinetically more readily accessible and may therefore represent a key precursor to protein aggregation. Therefore, they often tend to aggregate due to the formed hydrophobic forces when hydrophobic amino acid residues and regions of unstructured polypeptide backbones are exposed. Driven by intermolecular interactions, amorphous aggregates that lack long-range order form predominantly. Alternatively, aggregation can lead to the formation of ordered, fibrillar oligomers as seeds. The subsequent elongation is highly favourable and proceeds rapidly to the fibril structure.

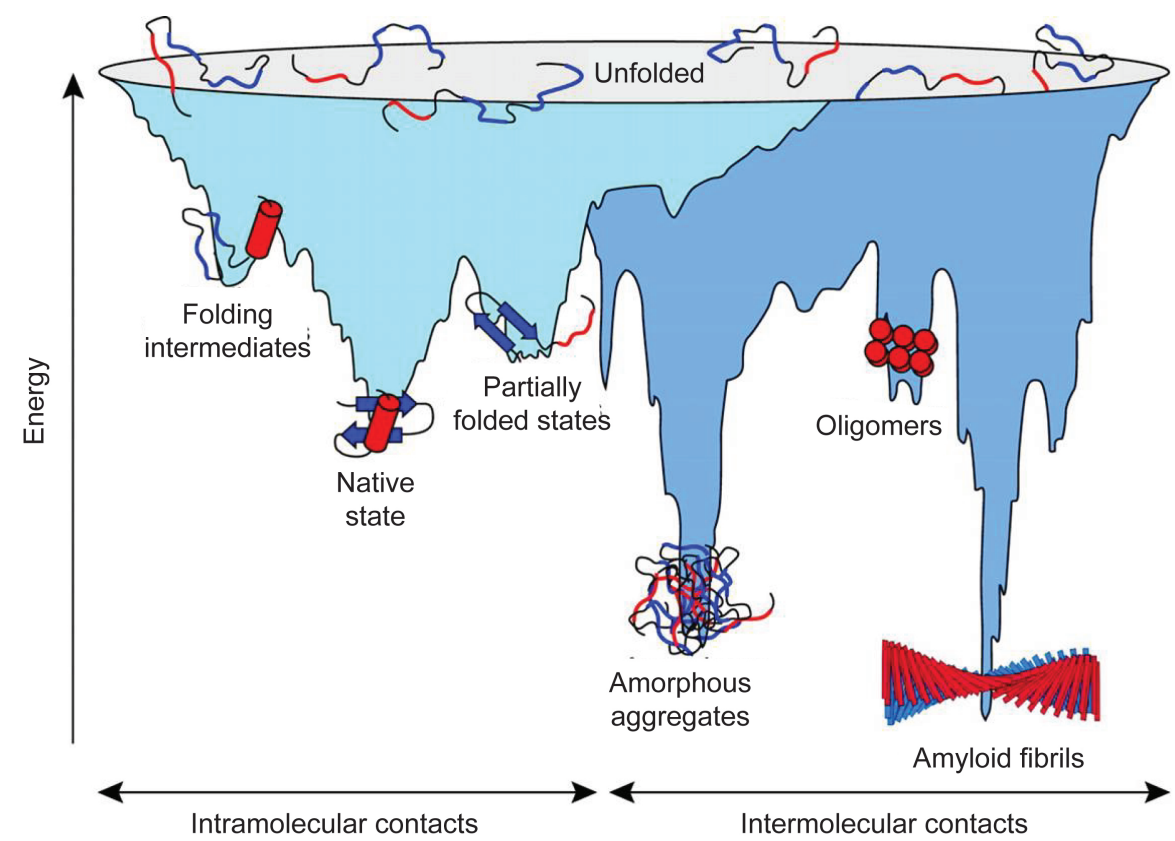

Figure 1.6: Schematics of energy landscape of protein folding and aggregation, taken from Vabulas et al. [252]. The light blue surface shows the possible conformations following the down-hill path due to the intramolecular contacts, and the dark blue surface shows the energy-favored states due to intermolecular contacts.

There is evidence that most of the protein mutations and denaturation associated with amyloid formation can result in a higher equilibrium population of the non-native unfolded and partially unfolded states. The cause could be a decrease in the conformational stability of the native fold [45]. In the presence of chaperons, the monomers can be more populated and stabilized as a result 
of the increase in free energy of activation from the native state to the unfolded state $[30,52,104]$.

\subsubsection{Diagnosis and Inhibition of Neurodegenerative Diseases}

Early diagnosis of neurodegenerative diseases and other amyloid-related diseases are of great importance. Until 1988, the only practical diagnosis of amyloidosis made use of the Congo Red staining assay. Later on, the use of radiolabelled serum amyloid P component (SAP) was developed into a routine tool in clinical practice as a specific, quantitative in vivo tracer for amyloid deposits, as SAP has been suggested to exist in amyloid deposits and undergoes specific calcium-dependent binding to amyloid fibrils [206]. The ThT assay has subsequently been introduced as diagnostic method for the diseases. In the more recent past, conformation-sensitive antibodies started to be used for diagnosis of neurodegenerative diseases [101].

Current clinical treatment of amyloidosis comprises maintenance of organ function, dialysis or transplantation and control of responsible conditions for production of fibril precursors [206]. Elimination of the supply of precursor proteins leads to regression of amyloid deposits. Based on the understanding of the amyloid formation mechanisms, new approaches under development are being directed towards achieving one of the following goals as illustrated in Figure 1.7 [52,102]:

Stabilization of the native folded structure. The native monomers can be bound with proteins or small molecules to prevent unfolding and aggregation.

\section{Inhibition or interference of steps related to conformational change.}

This strategy is to introduce binding molecules to individual steps to inhibit nucleation and elongation processes or to direct the paths towards non-amyloid products. The steps including formation of amyloid structured oligomers, protofibrils and also mature fibrils are involved here.

\section{Clearance or degradation of neurotoxic species or amyloid fibrils.}

Recently, structured oligomeric nuclei have been considered to be the primary cytotoxic species for neurodegenerative diseases [57, 223, 243], but whether amyloid fibril deposition itself may be a protective process is still under debate. Nonetheless, it is noteworthy that, during the therapy development, care needs to be taken not only to the effects of mature amyloid fibrils but also to that of the protofibrils and the neurotoxic oligomers. 


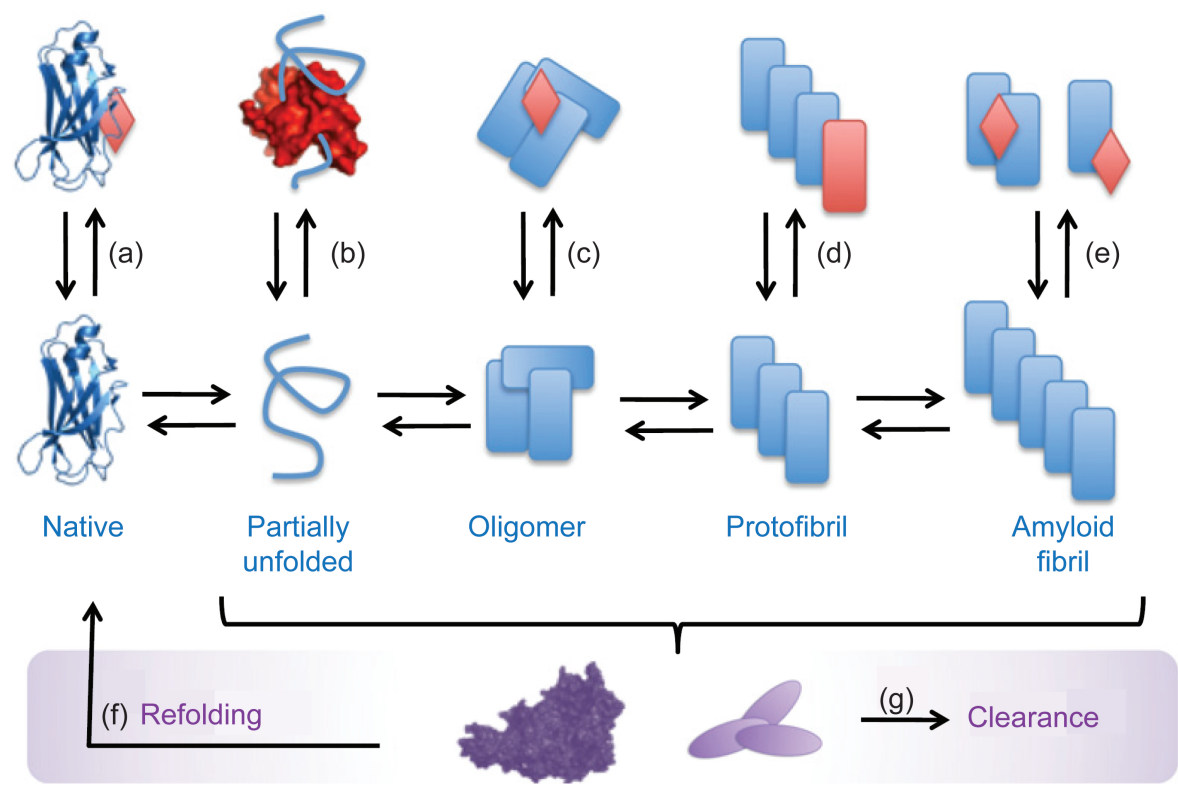

Figure 1.7: Schematic illustration of strategies to inhibit amyloid formation, taken from Hard et al. [102]. (a)-(e) Possible mechanisms by ligand binding leading to non-amyloid species. (a) Stabilization of monomers in native states. (b) Sequestering of monomeric peptide. (c) Off-pathway to non-toxic oligomers. (d) Off-pathway to terminate fibril elongation. (e) Disassembly of amyloid fibrils. (f) Refolding backwards to native monomer state. (g) Clearance of amyloid-related species.

Substantial effort has been given to find or develop drugs that accomplish the tasks above. Many species that inhibit the in vitro amyloid formation have been explored and tested using diverse biophysical techniques, although further evaluation of the candidates for therapeutic purposes is necessary. Some of the important candidates for which great therapeutic potential has been reported are summarized and listed in Table 1.2. They are categorized by six inhibition strategies:

Small aromatic molecules. Currently, many small aromatic molecules are suggested to be potential candidates for inhibition of amyloid formation. This class of molecules was shown to inhibit the amyloid fibril growth, possibly because their binding can stabilize the protein or in some cases alter the morphology of amyloid fibrils [158].

Small peptides or proteins. Short peptides, proteins and antibodies have been reported to achieve efficient inhibition of amyloid formation by specifically binding to a particular conformation of intermediates or protofibrils [86,101]. However, serious side effects have been shown to take place [2].

Chaperones. Chaperones have been proposed to stabilize misfolded proteins by 
Table 1.2: Inhibitors reported for neurodegenerative diseases.

\begin{tabular}{lll}
\hline \hline Category & Inhibitor & Protein \\
\hline Small aromatic molecules & Benzene, 1-naphthol, & Insulin \\
& Epigallocatechin gallate [158, 279] & \\
& Congo Red, ThT [32] & $\mathrm{A} \beta$ \\
& Metalloporphyrins [202,231] & Insulin, A $\beta, \tau$ \\
& Naphthalene-derivates [158] & Insulin \\
& A julolidine aldehyde [59] & $\mathrm{A} \beta$ \\
& Methylene blue [198] & $\mathrm{A} \beta, \tau$ \\
& PcTS [5, 217] & $\mathrm{A} \beta, \alpha \mathrm{S}, \tau$ \\
Small peptides/proteins & B10AP [101] & $\mathrm{A} \beta$ \\
& ZA $\beta 3$ Affibody [102] & $\mathrm{A} \beta$ \\
Chaperones/Proteases & $\beta$-/ $\gamma$-secretase [2,52,103] & $\mathrm{A} \beta$ \\
& Clusterin, SAP [268] & $\mathrm{A} \beta, \alpha \mathrm{S}$ \\
& sHsp $\alpha \mathrm{B}-$ Crystallin [138, 260] & $\alpha \mathrm{S}$, Insulin \\
& Chemical chaperone TMAO [138] & Insulin \\
Metal ions & Cu ${ }^{2+}$, Zn ${ }^{2+}[103,192]$ & $\mathrm{A} \beta$, Glucagon \\
Natural products & Ectoine, Betaine, & Insulin, Hutingtin \\
& Trahalose, Citrulline [9] & \\
Carbohydrates & Heparin [89] & Insulin \\
& Dextrose, Emdex [124] & Insulin \\
\hline \hline
\end{tabular}

binding to them followed by guiding their degradation. However, care must be taken here since chaperones can in some cases also promote amyloid formation [268].

Metal ions. Metal ions have been suggested to prevent amyloid formation by coordinating to the native monomers, and therefore serve as co-factors in modulating the amyloid formation. The strategy has been used for $\mathrm{A} \beta$ fibril inhibition and has now been reached the clinical trial stage [102].

Natural products. Some stress-induced molecules, such as ectoine, trahalose, betaine and citrulline, are accumulated in many microorganisms for surviving against external stress. These natural products show their ability to prevent abnormal protein folding at high concentrations (ca. $500 \mathrm{mM}$ ) due to increased protein stability [9].

Carbohydrates. Some carbohydrates have been reported to suppress protein aggregation into fibrils. Heparin, for example, has been proposed to bind to the positive domain of insulin as a strong polyanion and thereby to inhibit the 
electrostatic interaction within proteins [89].

\subsection{Detection Techniques for Amyloid Formation}

Techniques to detect and analyse amyloid formation are important for gaining insight into the amyloid characteristics, mechanistic and thermodynamic information introduced in Section 1.1.1 and Section 1.1.2. The most widely applied in vitro and in vivo technologies $[6,14,27,30,138,189,205,259]$ are summarized in Table 1.3. Only in vitro technologies are described in detail as follows.

\section{Techniques to reveal structure}

X-ray crystallography is used to determine amyloid structures of various states. The limitation is that proteins need to be crystallized prior to the detection [205]. Nuclear magnetic resonance spectroscopy (NMR) $[41,111,210,217]$ is a powerful spectroscopic technique to probe structures of species in protein aggregation pathways. Furthermore, NMR relaxation can be used to study dynamics of the aggregation events. In contrast to X-ray crystallography, crystallization is not necessary using this method. The conformational changes accompanying protein aggregation can also be detected using circular dichroism spectroscopy (CD) $[8,123,128]$, infrared spectroscopy (IR) and Raman spectroscopy [8, 14, 261] by revealing the protein secondary structures. In mass spectrometry, molecules are fragmented as molecular ions based on the mass-to-charge ratios [148,215]. Both mass spectrometry and NMR spectroscopy can measure dynamics of conformational fluctuations in proteins by conducting hydrogen-deuterium exchange experiments. The kinetic measurements using the mass spectrometry, CD, IR and Raman spectroscopy allow relative quantification, but cannot measure absolute rate constants of conformational changes.

\section{Techniques to detect morphology}

Separation methods including electrophoresis [212] and size exclusion chromatography (SEC) [230] are able to measure the size and mass distribution of molecules during amyloid formation. A significant limitation of these methods lies in the equilibrium disturbance during the separation, causing uncertainty and complexity in data analysis. The problem arises also in molecular-weight-dependent sedimentation and analytical ultracentrifugation (AUC) [205], which measures the 
change of mass distribution in amyloid formation processes.

In contrast, light scattering [146,230] or dynamic light scattering (DLS) [214] are more straightforward spectroscopic methods to observe the size distribution of the amyloid aggregates in different states. These label-free methods measure the hydrodynamic radius from $1 \mathrm{~nm}$ up to $4 \mu \mathrm{m}$ by detecting the fluctuations of the scattered light intensity. The fluctuations provide a measure of the molecular diffusion coefficient, which depends on the size and shape of the molecules. The main drawback of the detection system is sensitivity to large aggregates or impurities [27,230]. Furthermore, fibril-fibril interactions in solution may also lead to overestimation of the fibril size by slowing down diffusion. Finally, data analysis based on the autocorrelation function and interpretation can be quite complex and challenging with polydisperse samples.

The size distribution and molecular morphology can also be detected using ultrastructural methods with high resolution (subnanometer to nanomter scale). Electron microscopy (EM) has often been applied to long, unbranched fibrils. However, the method cannot be readily applied to monitor in situ amyloid growth, as heavy metal staining and drying of the samples is required prior to the detection $[43,205]$.

The 3D-structure (topography) of amyloid aggregates can be directly visualized in the ambient environment by atomic force microscopy (AFM) [1, 130, 171, 272]. The specimens are placed on an even surface and scanned with a cantilever with a nanometer radius-of-curvature-sized tip under piezoelectrical control. AFM detection provides height information with subnanometer resolution by recording the cantilever deflections in an optical lever system. Recently, time-lapse AFM studies have been carried out to study aggregate growth, and the method will be presented in the following Section 1.2. Compared to TEM, AFM has limitations in lateral resolution. 
Table 1.3: Techniques applied for amyloid formation.

\begin{tabular}{lll}
\hline \hline Method & Characteristic & Comment \\
\hline In vitro assays & & \\
\hline X-ray crystallography & Structure & Crystallization required; Limitation of size inhomogeneity. \\
CD spectroscopy & Secondary structure & Label-free; Relative quantification; Large sample amount. \\
NMR spectroscopy & Structure \& dynamics & Label-free. \\
Mass spectrometry & Structure \& Kinetics & Relative quantification. \\
IR and Raman spectroscopy & Structure & Relative quantification. \\
TEM microscopy & Size \& Morphology & Quantitative analysis challenging; Sample dried and at- \\
& & tached to a solid support. \\
AFM microscopy & Size \& Morphology \& Kinetics & Label-free; Low throughput; Sample attached to a solid \\
& & support. \\
SEC & Size \& Shape & Lengthy experiment; Equilibrium disturbance. \\
AUC & Molecular weight & Large sample amount. \\
Light scattering & Size & Label-free; Low sensitivity to aggregation forms. \\
DLS & Size \& Kinetics & Label-free; Labor intensive; Low sensitivity to aggregation \\
& & forms. \\
Electrophoresis & Size \& Shape \& Charge & Solubility required; Equilibrium disturbance. \\
In situ ThT assay, TIRFM & Fluorescence \& Kinetics & Interaction of ThT with aggregates. \\
Ex situ ThT assay & Fluorescence \& Kinetics & More labor-intensive; Artifacts disturbance. \\
SPR & Mass \& Kinetics & Label-free biosensor; Surface-bound detection. \\
QCM & Mass \& Kinetics & Label-free biosensor; Surface-bound detection. \\
\hline In vivo assays & & \\
\hline MTT assay & Cell death & Lengthy experiments \\
Immunohistochemistry & Aggregate amount \& Location & Specific antibodies required. \\
ELISA assay & Aggregate levels & Specific antibodies required. \\
Genetic screening & Pathological pathways & Lengthy experiment. \\
\hline \hline
\end{tabular}




\section{Techniques to monitor kinetics}

Many techniques to measure kinetics of amyloid growth are performed in bulk solutions. Traditional methods rely on staining with dyes such as Congo Red, ANS or ThT. Binding of such dyes to the amyloid fibrils changes their spectral properties and can thereby be detected with optical spectroscopic methods $[11,20,129,178,219]$. Currently, the fluorescence-based ThT assay is widely applied as a gold standard to measure the kinetics of amyloid formation. Figure 1.3 shows a typical time course of amyloid growth detected by ThT. However, these dyes are insensitive to oligomers and other early aggregation species. Furthermore, dyes are very likely to interfere with the aggregation process. For example, Congo Red has already been suggested to inhibit in vivo amyloid formation $[155,276]$ in certain environments. ThT has also been reported to interfere with the amyloid formation during binding with the fibrils and the measured signals can be biased [32,115, 192]. Further problems include indeterminate binding stoichiometry and the insensitivity to oligomeric intermediates. In general, quantitative kinetic measurements in solution are challenging due to the inaccuracy of the exact concentrations of the species [20,265].

Recently, much effort has consequently been directed towards developing quantitative surface-based detection techniques, which allow real-time monitoring of fibril growth in the elongation phase. The most significant methods reported to detect the process are in situ AFM, TIRFM, QCM and SPR. Schematics of the working principles and the characteristic growth curves are summarized and depicted in Figure 1.8. In all four approaches, seed fibrils are immobilized on the surface of the detection systems prior to the exposure to the monomer solutions.

In situ AFM. AFM experiments in liquid enable time-lapse measurements of biological samples in the aqueous environment. The specimens are fixed here on the substrate surface and the cantilever tip maps the height of the surface using tapping mode. The scan speed is several seconds or minutes per frame. The approach has been recently reported to monitor the in situ growth of amylin protofibrils, as shown in Figure 1.8 (b), and fibrils formed with specific heptapeptides [1,97].

TIRFM. Total internal reflection microscopy uses an evanescent wave, generated by the total internal reflection of the incident light at the solid-liquid-interface, to selectively illuminate and excite fluorophores of the specimens in a restricted region adjacent to the interface. The evanescent electromagnetic field decays 
exponentially from the interface and penetrates to a depth of a few hundred nanometers into the medium. TIRFM has been applied to monitor amyloid elongation combined with ThT-staining assay. As shown in Figure 1.8 (a), Ban et al. observed a unidirectional length increase of $\beta 2-\mathrm{m}$ and $\mathrm{A} \beta$ seed fibrils lying in parallel with the slide glass surface, since the evanescent field formed by the total internal reflection of laser light penetrates to a depth of $150 \mathrm{~nm}$ [11,12].

QCM. QCM monitors the resonance frequency of surface shear waves on the sensor $[31,138,265]$. The rate of the frequency shift is proportional to the rate of mass change on the quartz crystal surface. In addition to the frequency measurements, the dissipation is often measured to quantify the damping in the system, as applied in the QCM-D detection system. The chamber volume above the sensor is $5-50 \mu \mathrm{L}$ and the minimum volume required for one sample injection is $30-150 \mu \mathrm{L}$. The mass sensitivity in liquid can be $0.5-2 \mathrm{ng} / \mathrm{cm}^{2}$. Typical flow rates are in the range of 50-200 $\mu \mathrm{L} /$ minutes. Knowles et al. determined the elongation rates of bovine insulin fibrils by measuring the increase in hydrodynamic mass bound to the quartz crystal, as shown in Figure 1.8 (c).

SPR. In SPR measurements, a polarized incident light beam excites the oscillation of electrons at the interface of metal and an aqueous solution [265]. When the frequency of photons of the incident light matches the natural frequency of the surface electrons, resonance takes place. At a specific incident angle, the intensity of the reflected light is reduced due to the energy transfer from the incident wave to surface plasmons. The resonance condition of SPR measurements is sensitive to the materials adsorbed onto the chip surface. The SPR signal, expressed in resonance units, is a measure of refractive index and thus of mass concentration within ca. $300 \mathrm{~nm}$ of a sensor chip surface. This implies that the approach can detect the growth of the immobilized amyloid fibrils less than $300 \mathrm{~nm}$ from the surface. The SPR signal increases when the immobilized fibrils (e.g. A $\beta$ in Figure 1.8 (d)) grow in the presence of monomer solutions and decreases when the fibrils degrade $[39,105]$.

Among these methods, in situ AFM [12,21,113] monitors the growth by length directly and TIRFM monitors the growth by length using ThT-assay. Moreover, biosensor-based methods, QCM [30, 31, 138, 196] and SPR [39, 105, 241] have been developed and applied as label-free techniques, that are sensitive to mass change on the biosensor surfaces as a consequence of the growth or degradation of the immobilized surface fibrils. These label-free biosensor methods have several advantages over conventional in-solution assays and have therefore gained increasing interest for the last several years [265]. First, small differences in elongation rates 
due to subtle variations in the reaction conditions can be resolved quantitatively in real-time. Second, the system allows probing the growth of a defined ensemble of fibrils repeatedly under various conditions simply by replacing the monomer solutions. Finally, both continuous flow cell systems keep concentrations constant over extended reaction times. Additionally, these platforms also allow the influence of disparate conditions and additives on the kinetics of amyloid formation to be studied quantitatively. So far, biosensor-based detection systems are still under development. However, both QCM and SPR are limited in terms of their potential for further miniaturization.

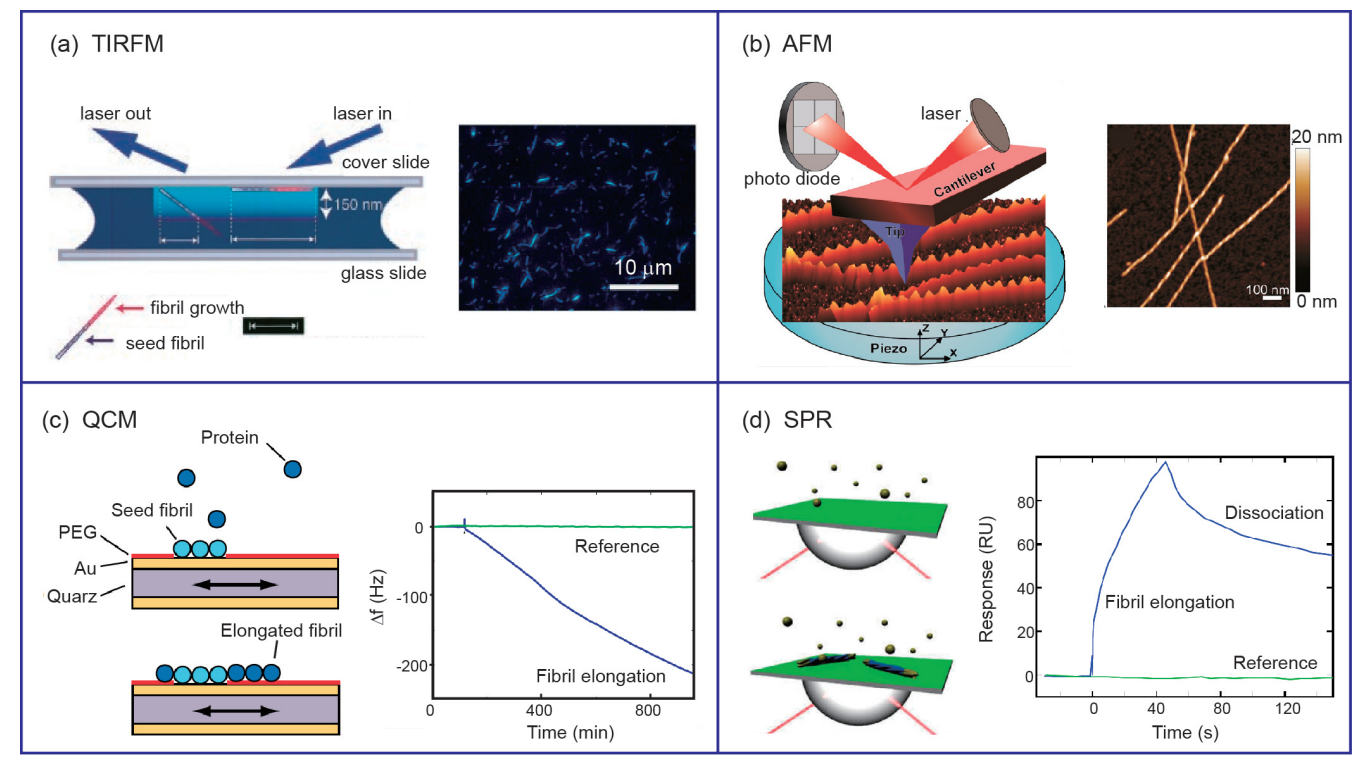

Figure 1.8: Working principles and the characteristic signals of the most significant surface-based techniques [(a) TIRFM (adapted from Ref. [11] and [12]), (b) AFM (adapted from Ref. [1]), (c) QCM (adapted from Ref. [138]) and (d) SPR (adapted from Ref. [265]] to study real-time amyloid fibril growth.

\subsection{Motivation and Aim of the Thesis}

The primary aim of this study is to develop a new surface-based approach with Suspended Microchannel Resonators (SMR) for precise online kinetic measurements of amyloid formation.

The SMR method measures the resonance frequency of a micromechanical resonator, that encloses a microchannel of $3 \times 8 \mu \mathrm{m}$ (Figure 1.9), and the frequency shift is correlated to changes of the resonator mass. The accuracy of the label-free approach relies on the high sensitivity of the resonance frequency of the SMR 
system to mass [34]. More details of the SMR technique and its characteristics are presented in the Theory chapter (Section 2.1.1).
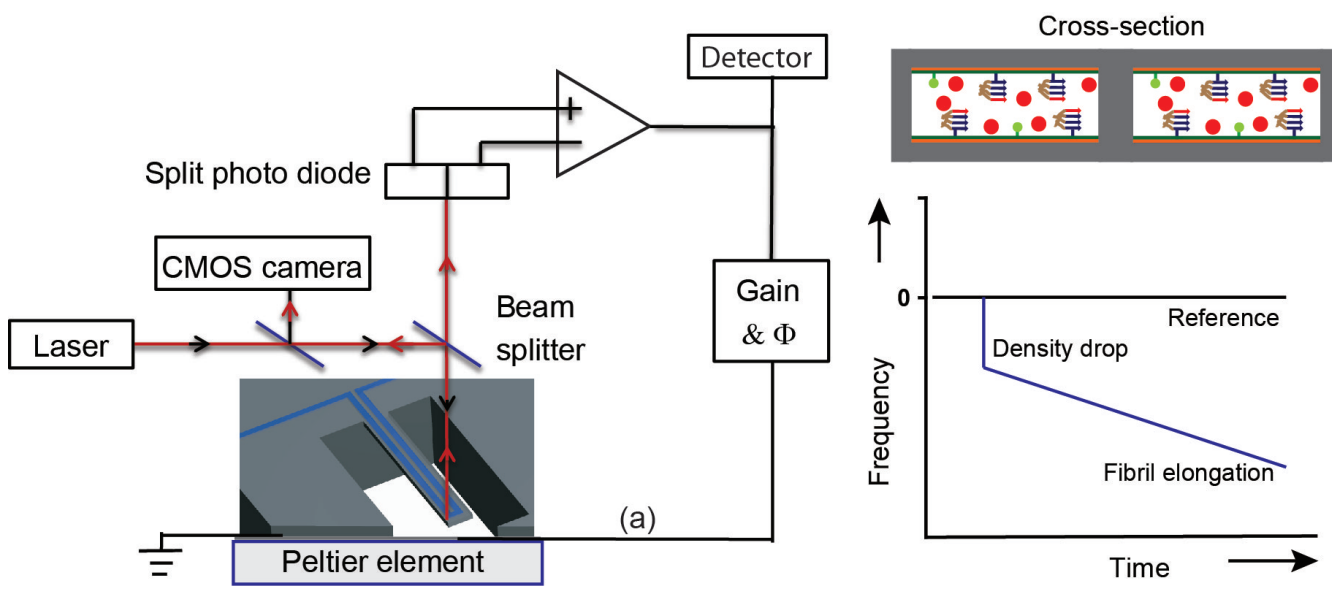

Figure 1.9: Working principle (adapted from Ref. [34] and [33]) and illustrated characteristic signals of amyloid growth detected by SMR (presented in the Kinetic Measurements chapter).

Although the resolution of the promising microfluidic transducer system is as high as $10^{-15} \mathrm{~g}$ in solution, the mass change induced by single monomeric proteins can still not be detected. The buoyant mass of insulin (51 AA), $\alpha \mathrm{S}$ (140 AA) and $\mathrm{A} \beta$ (42 AA) are all in the zeptogram range, which is over six orders of magnitude below the mass resolution of the SMR devices used in this work. In order to reveal kinetic and thermodynamic parameters governing the elongation phase of amyloid growth, the strategy has to be directed to the growth monitoring of a quantity of preformed seed fibrils that are immobilized on the inner walls of the microchannels. The fibril growth rates of the surface seed fibrils are then measured when monomer solutions flow through the microchannels.

The surface-based SMR method is presented here to be an alternative approach to the most currently reported biosensor techniques, but achieves additionally further miniaturization of the devices with higher mass resolution.

Compared to the conventional methods discussed in Section 1.2, the SMR approach has the following advantages:

- This label-free method is sensitive to all states of the aggregation and avoids the risk of interfering with the aggregation reaction.

- SMR enables in situ and real-time quantitative measurements of kinetics with high accuracy. 
- The surface-bound detection system allows successive measurements on a defined ensemble of molecules.

- The elongation detection of the immobilized fibrils is insensitive to the distance from the surface.

- The microfluidic system allows changing reaction conditions quickly and reliably by replacing the solutions in microchannels. This provides the practical benefit of high sample throughput.

- The detection with the miniaturized devices can be performed within minutes with sample consumption as low as several tens of microliters.

Compared to SPR and QCM with sensor dimensions in the millimeter range, the surface-based SMR approach with the resonator microchannel of ca. $5.6 \mathrm{pL}$ allows measurements with low sample consumption over a large time scale from seconds to hours. Furthermore, in the surface-based SMR measurements, mass resolution approaches $0.01 \mathrm{ng} / \mathrm{cm}^{2}$ and is still being optimized. In contrast, the mass resolution is ca. $0.5-2 \mathrm{ng} / \mathrm{cm}^{2}$ in QCM measurements and ca. $0.05 \mathrm{ng} / \mathrm{cm}^{2}$ in SPR measurements.

Reliable immobilization of seed fibrils onto the inner walls of SMR microchannels is among the most important prerequisites for the measurement. However, this task is challenging in such microchannels of micrometer dimensions. First, contaminations need to be strictly eliminated, since they can easily clog the microchannels. Thereafter, the inaccessibility of the surfaces increases the difficulty of performing multi-step protein immobilization inside the microchannels. Finally, evaluation methods for the microchannel functionalization and immobilization need to be developed, since the microchannels of SMR devices in this work are neither accessible to surface analytical tools nor visible.

The scientific objectives of the dissertation comprise three parts as follows:

\section{Development of methods for surface functionalization and protein immobilization inside microfluidic devices}

- Development of quantitative methods to evaluate the surface functionalization and protein immobilization.

- Functionalization of microchannel surface inside the SMR devices. 
- Stable and reproducible protein immobilization in SMR devices to allow further kinetic measurements.

- Blocking of non-specific protein binding.

Preparation and characterization of defined seed fibril ensembles for quantitative measurements

- Establishment of defined ensembles of seed fibrils with a model protein system.

- Evaluation of homogeneity of seed fibril size.

- In situ evaluation of surface density of immobilized seed fibrils inside SMR devices.

\section{Kinetic measurement and modeling of amyloid elongation using SMR}

- Kinetic monitoring of the elongation process of the model protein.

- Interpretation of mechanism and thermodynamics of the elongation process based on data analysis of kinetic measurements.

- Evaluation of influence of environmental factors (temperature, concentration, shear stress, salt concentration etc.) on amyloid elongation kinetics.

- Exploration of potential inhibition or degradation candidates for amyloid elongation based on kinetic measurements. 


\section{Chapter 2}

\section{Theory}

In the first part of this chapter (Section 2.1.1), the concept of the SMR devices is presented to introduce the principle, detection modes and characteristics of the microfluidic transducer. Thereafter, representative surface modification techniques are reviewed in the Section 2.2 for protein immobilization and surface passivation inside silicon/glass-based microfluidic devices.

\subsection{Microfluidic Devices}

Microfluidics is a field of miniaturized systems that make use of channels with micrometer dimensions to manipulate small amounts (less than several microliters) of fluids [254,266]. Over the last decade, large varieties of analytical and diagnostic microfluidic devices have been constantly developed in life science laboratories as they provide many advantages in terms of low sample consumption, liquid handling precision and throughput $[16,22,50,64,71,262]$. An important benefit of using such devices is that fluid manipulation with small volume (a few picoliters to microliters) can lead to measurements with fundamentally higher precision [242].

A variety of methods exist to fabricate microfluidic devices. The choice of the method is determined by factors such as cost, speed, feature size and material $[77,270]$. In recent years, polymer-based microfluidic devices fabricated by soft lithography have widely emerged due to their low cost, rapid and prototypefriendly fabrication, ease of use and disposability. Polydimethylsiloxane (PDMS) and poly(methyl methacrylate) (PMMA) are currently the two most widely used polymers. However, traditional silicon- or glass-based microfluidic devices, which originated and developed from the semiconductor industry, benefit from mature high-resolution microfabrication techniques and will continue to be utilized in 
many applications. Moreover, glass and silicon are well-known as chemically inert materials. The initial prototypes of the SMR devices were packaged using PDMS microfluidics, but the shortcomings of air permeability, compliant characteristics and limited chemical resistance make robust vacuum packaging of the SMR devices challenging for optimal performance [35]. Silicon-based microfluidic devices are therefore more applicable for this purpose. Furthermore, besides the capability for batch-fabrication, these silicon-based devices are chemically inert and have been demonstrated to have a shelf life of several years.

\subsubsection{Suspended Microchannel Resonators (SMR)}

The SMR devices are mass-sensitive microelectromechanical systems (MEMS) and were first developed to weigh single nanoparticles, single cells and adsorbed layers of biomolecules in fluid $[34,93,153]$. With a variety of geometries as shown in Figure 2.1 (b and c) [33,263], the SMR devices have been integrated with different techniques such as fluorescence microscopy [236], a pre-concentration element [62] and surface charge measurement [61]. The method has been implemented for many applications. To date, the capabilities of these devices for biological and physical detection have been used to study cell growth [29, 94, 236, 263], cell density [99], cancer biomarkers [255], dispersion or agglutination of microspheres [49,61,93,203], and characteristics of binary mixture systems [151,152]. In the current study, the SMR devices are employed in combination with surface immobilization techniques to explore the kinetics, mechanisms and thermodynamics of amyloid growth.

\section{Principles of SMR}

An SMR device comprises a vacuum-packaged free-standing resonator, which encloses a $3 \mu \mathrm{m}$ high and $8 \mu \mathrm{m}$ wide microfluidic channel to allow fluid flow, as shown in Figure 2.1(a). The resonator is driven into mechanical resonance using a positive feedback loop (Figure 1.9 in Section 1.2). An optical lever detection system is applied to measure the displacement signal of a reflected laser beam from the tip of the resonator. In the oscillation circuit, the same signal is amplified, phase shifted into a positive feedback and connected back to the biased drive electrode.

The resonance frequency shifts when the total mass of the SMR resonator changes, and this shift is recorded with high accuracy by measuring the difference between the SMR signal and a manually adjusted reference signal. When damping 
(a)

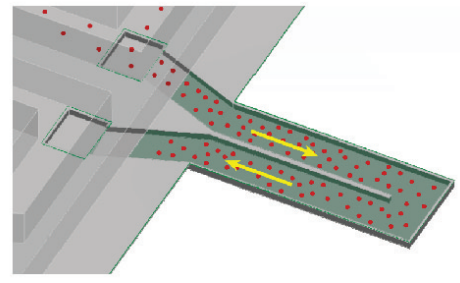

Side-view of SMR

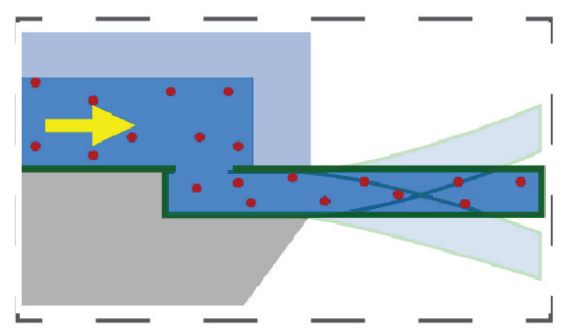

Buffer (low pressure) Buffer (high pressure)

(b)

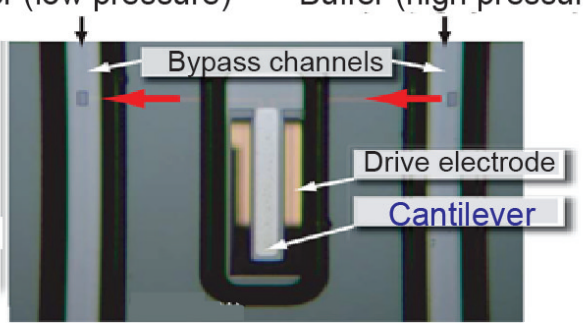

(c)

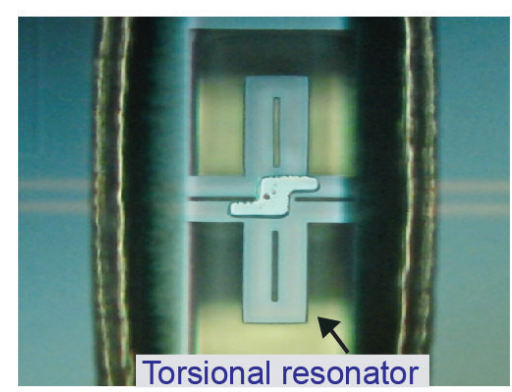

Figure 2.1: Schematics of SMR devices. (a) Working principle of SMR detection. Topview of (b) cantilever-shaped and (c) torsional resonators.

is low, the frequency $f_{0}$ of the resonator with effective mass $m^{*}$ is given by:

$$
f_{0}=\frac{1}{2 \pi} \sqrt{\frac{k}{m^{*}}}
$$

With the embedded microchannel inside resonator, fluid can fill the device and contributes to the total effective mass $m^{*}$ in addition to the resonator itself. A mass change $\Delta \mathrm{m}$ in the fluid or at the solid-liquid-interface results in a new resonance frequency $f$, as described by Equation 2.2:

$$
f=\frac{1}{2 \pi} \sqrt{\frac{k}{m^{*}+\alpha \Delta m}}
$$

where $\alpha$ is a geometry-related parameter. The value of $\alpha$ depends on the geometric localization of the added mass $\Delta m, \alpha \approx 1$ when a particle in transit is located at the tip of a cantilever-shaped microchannel, and $\alpha \approx 0.24$ when the mass inside the resonator channel increases uniformly [33]. When molecules are adsorbed to the microchannel walls, the total mass is composed of the fixed mass of the resonator, the fluid inside the channel and the adsorbed molecules at the solid-liquid-interface. Here, the mass of the molecules refers to the buoyant mass $m_{\text {buoyant }}$, which depends on the density $\rho_{\text {molecule }}$ : 


$$
m_{\text {buoyant }}=m_{\text {dry }} *\left(1-\frac{\rho_{\mathrm{H}_{2} \mathrm{O}}}{\rho_{\text {molecule }}}\right)
$$

where $\rho_{\mathrm{H}_{2} \mathrm{O}}$ and $m_{d r y}$ are the density of water and the molecule mass in air. As the density of proteins (monomers and fibrils) is greater than that of water, the frequency decreases with the accumulation of molecules at the solid-liquid-interface.

\section{Detection modes}

Depending on various applications of the SMR devices, two types of detection modes can be used, as listed below:

Flow-through mode as shown in Figure 2.2 (a). Applying this mode, the devices can be used to measure the mass of particles that are suspended in the fluid. When particles flow through the resonator, peak signals can be obtained with peak heights directly proportional to the mass of the particles. Also, the resultant frequency shift depends on the mass and position of the particles [34].

Surface-based SMR measurements as shown in Figure 2.2 (b). Channel surfaces are modified and functionalized to enable molecule adsorption at the solid-liquid interface. As the ratio of surface area to volume of the SMR device is large $\left(10^{4} \mathrm{~cm}^{-1}\right)$, a large quantity of molecules can be adsorbed to the inner walls of the channels. The total mass change of the adsorbed molecules can thus be measured as a result of frequency shift.

(a) Flow-through mode

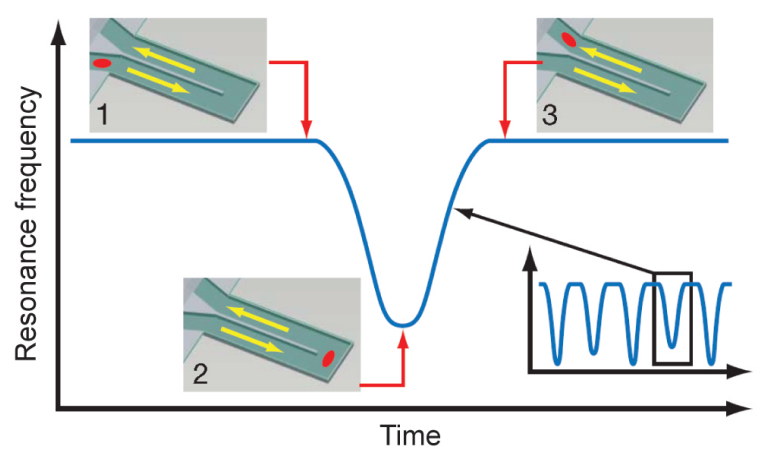

(b) Surface-binding assay

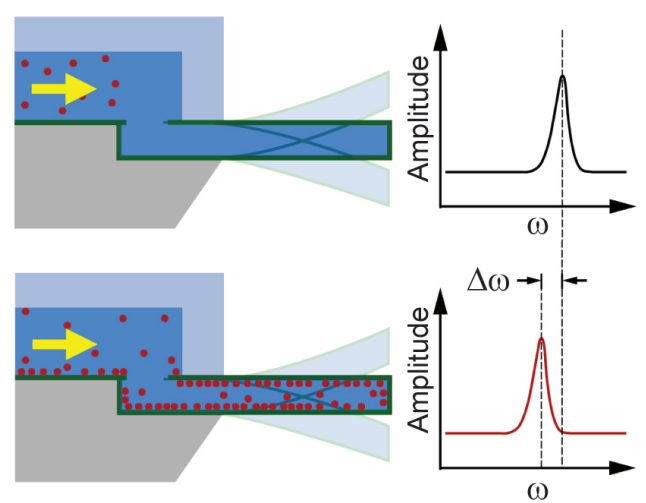

Figure 2.2: Two detection modes of SMR measurements: (a) flow-through mode and (b) surface-based SMR measurements [34,36].

In this thesis, surface-based SMR measurements are performed to measure the precise kinetics of the amyloid elongation process. The reliability and stability of 
the detection system will be presented in Section 5.2.

\section{Sensitivity}

In surface-based SMR measurements, the total mass change due to a large number of adsorbed molecules is detected. This approach is advantageous due to the fact that the mass change can be monitored over an extended time interval until the signal is significantly above the noise floor. Furthermore, by applying proper methods to immobilize molecules and passivate the surfaces, the total mass increase of bound molecules at the solid-liquid interface is measured and false response arising from nonspecific molecule adsorption can be efficiently reduced [7]. As such, the sensitivity requirement for the surface-based SMR measurements is less stringent compared to the flow-through mode, by which the mass of single molecules would need to be resolved [33].

The mass sensitivity (mass per unit area) of the surface-based SMR measurements is relevant for the kinetic measurements. With constant channel cross-section, the sensitivity is independent of the exact geometry and vibrational mode of the channel $[33,36]$. Therefore, the mass sensitivity can be estimated by referring to a double clamped beam with geometric parameters illustrated in Figure 2.3.

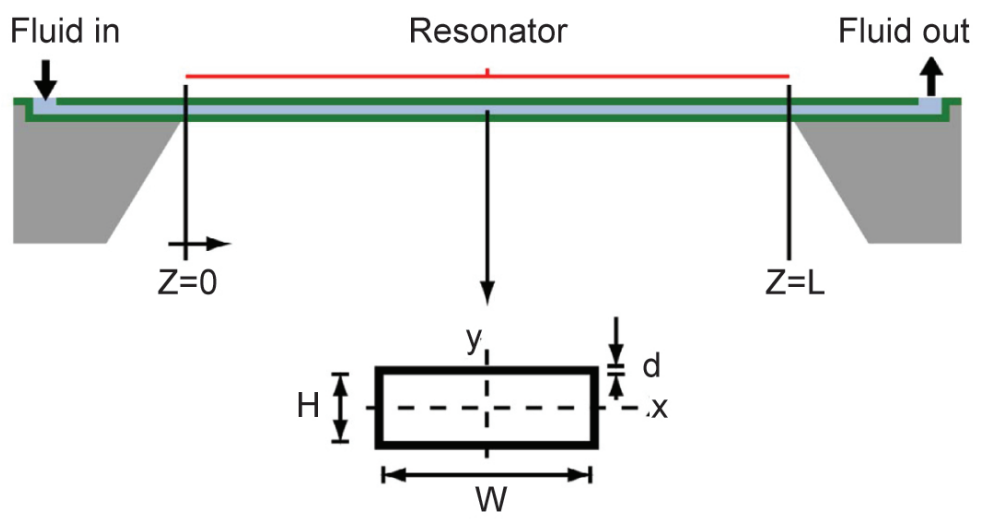

Figure 2.3: A double clamped beam to illustrate the mass sensitivity of an SMR device, taken from reference [33] and [36].

To obtain the sensitivity in surface-binding assays, the resonance frequency $\omega_{0}$ $\left(\omega_{0}=2 \pi f_{0}\right)$ can also be expressed by Equation 2.4 based on the Rayleigh-Ritz principle, 


$$
\omega_{0}^{2}=\frac{U}{\int_{0}^{L} \frac{1}{2} m_{A}(z) \cdot u(z)^{2} d z}
$$

where $U$ is the potential energy of the displacement field $u(z)$ and $m_{A}(z)$ is the mass per unit length, expressed as:

$$
m_{A}(z)=\int \rho(x, y, z) d x d y
$$

Uniform molecule adsorption on the channel walls is assumed to only change the maximum kinetic energy by added mass but not influence the maximum potential energy of the resonator. A small mass increase $\Delta m_{A}$, giving rise to a frequency shift $\Delta \omega$, can be approximated as follows:

$$
\frac{\Delta \omega}{\omega_{0}} \approx-\frac{1}{2} \frac{\int_{0}^{L} u(z)^{2} \Delta m_{A}(z) d z}{\int_{0}^{L} u(z)^{2} m_{A}(z) d z} \approx-\frac{1}{2} \frac{\Delta m_{A}}{m_{A}}
$$

Equation 2.6 can be further rewritten into equation 2.7 with defined mass per unit length of the solid-liquid boundary, $\frac{m_{A}}{s}$, in equation 2.8 :

$$
\frac{\Delta \omega}{\omega_{0}} \approx-\frac{1}{2} \frac{\Delta m_{A}}{s}\left(\frac{m_{A}}{s}\right)^{-1}
$$

and

$$
s=2(H+W)
$$

where $\mathrm{H}$ and $\mathrm{W}$ are the height and width of the channel.

Equation 2.7 shows the relevance of the parameter of $\frac{m_{A}}{s}$ for the mass sensitivity. A decrease in $\frac{m_{A}}{s}$ will result in enhanced sensitivity to surface adsorbed mass.

In Equation 2.6, the ratio $\frac{\Delta \omega}{\omega_{0}}$ only depends on the ratio of $\frac{\Delta m_{A}}{m_{A}}$. The mass sensitivity can thus be optimized by reducing the resonator mass and by maximizing molecules adsorbed to the channel surface. In the surface-based measurements, the frequency resolution of the high quality resonator employed here is approximately $0.01 \mathrm{ppm}$ in a $1 \mathrm{~Hz}$ bandwidth. With the known SMR channel dimensions (height $\mathrm{H}=3 \mu \mathrm{m}$ and width $\mathrm{W}=8 \mu \mathrm{m}$ ), the wall thickness (top and bottom: $\mathrm{d}=2 \mu \mathrm{m}$; outside: $\mathrm{d}_{\text {out }}=6 \mu \mathrm{m}$; between the channels: $\left.\mathrm{d}_{i n}=2 \mu \mathrm{m}\right)$ and the silicon density $\left(\rho_{S i}=2.336 \mathrm{~g} / \mathrm{cm}^{3}\right)$, the minimal detectable surface density $\sigma$ of molecules adsorbed to the surface can be predicted to be ca. $0.004 \mathrm{ng} / \mathrm{cm}^{2}$ using Equation 2.9. 


$$
\frac{\Delta m_{A}}{m_{A}} \approx \frac{\sigma}{\frac{1}{2} \rho_{S i} \frac{\left(W+d_{\text {out }}+d_{\text {in }}\right)(H+2 d)}{(W+H)}+\frac{1}{2} \rho_{H_{2} O} \frac{W H}{(W+H)}}
$$

The sensitivity of the SMR can also be measured by calibrating the device using fluids of different density. Density sensitivity, $S_{\rho}$, can be obtained by linear regression using Equation 2.10, which is valid for small density changes $\Delta \rho$ :

$$
\frac{\Delta \omega}{\omega_{0}}=-S_{\rho} \cdot \Delta \rho
$$

The frequency shift per adsorbed mass, $S_{\text {Surface, }}$ can then be estimated using Equation 2.11,

$$
S_{\text {Surface }}=S_{\rho} \cdot\left(\frac{H \cdot W}{L}\right)^{-1}
$$

The mass resolution can thus be determined as the product of the frequency resolution and the reciprocal of $S_{\text {Surface }}$. The mass resolution of the surface-based SMR measurements has been reported to be $0.01 \mathrm{ng} / \mathrm{cm}^{2}$ [34] and can be further optimized by reducing the resonator mass and increasing the adsorbed molecule density. Compared to other mass-sensing methods such as QCM and SPR, the mass resolution of the SMR devices has been improved by at least five times. Further shrinking of the SMR channel size to sub-micrometer dimension may yield even higher sensitivity.

\section{Other characteristics of SMRs}

Characteristics of the SMR devices, such as quality factor and drift of the detection, are important parameters which influence the reliability of surface-based measurements. In the current thesis, long-term stability and a high quality factor are important for kinetic measurements of amyloid fibril growth. As presented in previous studies, relevant characteristics have also been assessed [33,34,37]:

Quality factor (Q-factor). High Q-factors (ca. 15,000) can be achieved [33,34, $263]$ with dry resonator channels of the SMR devices. Compared to conventional biosensors using micromechanical resonators [16, 26, 81,83, 204,278] that are immersed in the fluid, both sensitivity and Q-factor of fluid-filled SMRs are significantly elevated by diminishing high damping and friction drag caused by the viscosity of fluids. Non-monotonic energy dissipation has been experimentally observed and modeled in terms of viscosity and compressibility of the 
fluid. For specific geometries, no difference in quality factor can be observed between an air-filled and a water-filled resonator [36].

Drift of the detection. Temperature fluctuations change the resonance frequency, since temperature can affect the stiffness and stress of the resonator and the density of the fluid. It has been found that the resonance frequency decreases as temperature increases [33]. Therefore, it is important to stabilize the temperature during the measurements for more accurate detection [152].

\subsection{Protein Immobilization Techniques inside Mi- crofluidic Devices}

In microfluidic channels, the large surface-area-to-volume ratio $\left(10^{4} \mathrm{~cm}^{-1}\right)$ makes surface-based detection with a large number of immobilized molecules on the surface a powerful biosensing technique. In these measurements, high quality of the protein immobilization is a prerequisite for good detection performance and high accuracy $[10,13,47,84,125,141,154,160,186,208,216,227]$. Key performance parameters to evaluate the immobilization include surface coverage/density, longterm stability and reproducibility. Immobilization methods vary largely with the targeted surfaces, protein characteristics and aimed application.

Surface functionalization, the generation of reactive functional groups on the surface, is the critical step prior to the protein immobilization step. Functionalization leading to planar or three-dimensional (3D) protein immobilization can be conducted using different linkers, as shown in Figure 2.4. In contrast to planar immobilization with short-length crosslinkers (e.g. aminosilanes, epoxysilanes), polymeric hydrogels such as polyacrylamide gel (PA), dendrimers, polyethylene glycol gel (PEG) and dextran are used to form 3D linker matrices with a large number of protein binding sites [114, 133, 222, 253]. This provides a high protein capture capacity which can improve detection sensitivity compared to planar immobilization. Furthermore, hydrogels with long spacers can provide a hydrophilic environment which favors protein stability and allows proteins to retain their activity.

For many specialized biosensing methods such as SPR and QCM, proteins are immobilized on metal films deposited on glass or quartz surfaces. The corresponding immobilization techniques have been well developed and documented so far [108]. 


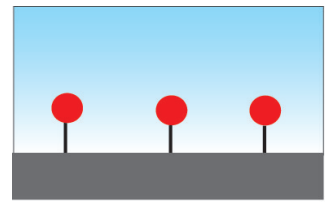

Planar structure

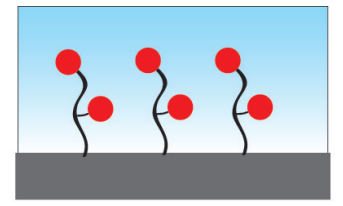

3D structure

(Single layer)

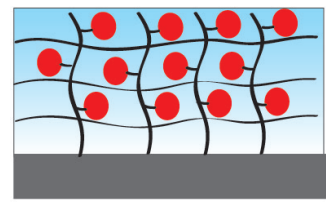

3D structure

(Multilayer matrix)

Figure 2.4: Schematic drawing of protein immobilization with different binding geometries such as planar structure and 3D structures.

For example, thiol-based chemistry is often applied for protein immobilization on noble metal surfaces including gold, silver and platinum [133]. As the SMR and other microfluidic devices used in this thesis do not make use of such surfaces, the related protein immobilization techniques will not be discussed here. In contrast, possible immobilization techniques will be reviewed in detail for silicon, glass and PDMS-polymer surfaces. All these surfaces share a similar surface chemistry, therefore the applied immobilization techniques are similar. Large varieties of immobilization methods are categorized into covalent coupling, physisorption, bioaffinity interaction and their combination as illustrated in Figure 2.5 [133].
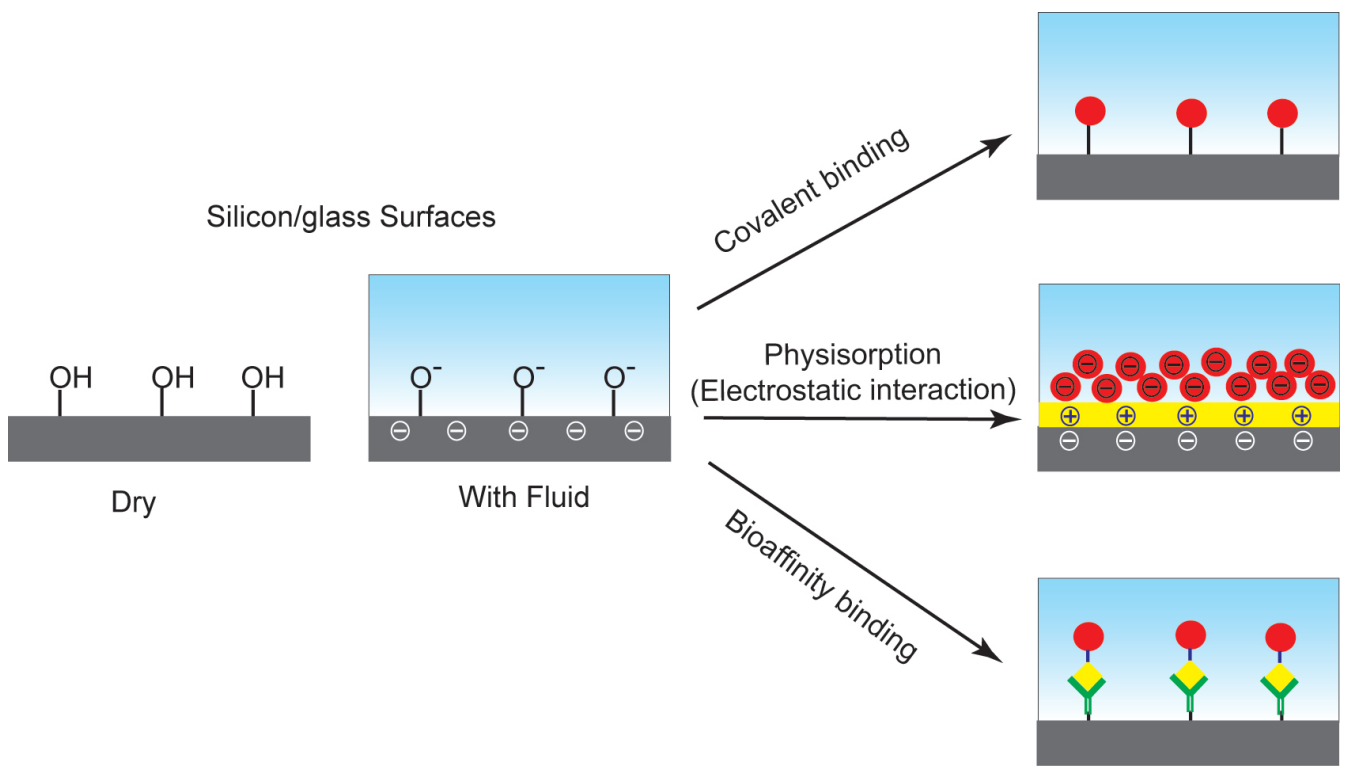

Figure 2.5: Schematic drawing of protein immobilization concepts via covalent coupling, phsisorption (e.g. electrostatic interaction) or bioaffinity interaction.

\subsubsection{Covalent Coupling}

Covalent coupling is frequently used to immobilize proteins with accessible functional groups of exposed amino acids. Covalent bonds are irreversible and in 
most cases stronger than other intermolecular interactions and bioaffinity interactions. Depending on the functional groups that are used to modify the surface or to link the targeted proteins, multiple surface functionalization steps are required prior to the protein immobilization step. Here, the conjugation chemistries which can be employed in silicon/glass-based microfluidic devices are reviewed [222]. Starting from the functionalization of the native solid surface, possible immobilization pathways are summarized in Figure 2.6 and Table 2.1.

Typically, microchannel surfaces with naturally or artificially grown silicon dioxide are initially functionalized using silane chemistry [91]. The applied organosilanes usually contain a functional group (e.g. amine, aldehyde, epoxy groups) at one end and a surface reactive group (e.g. alkoxy and chloro groups) at the other. In this study, aminosilanes (APTMS and APDMMS) with surface-reactive alkoxy groups were in use. Various protocols for aminosilane deposition have been reported on open-surface systems [108]:

Liquid-phase deposition. The liquid-phase deposition of amino alkoxysilanes has been accomplished in either organic solvents (e.g. toluene, acetone, hexane etc.) or aqueous solvents (e.g. water) [91, 108]. The suggested mechanism is presented in Figure 2.7. In both solvents, the alkoxy groups of the silanes, e.g. the methoxy groups of APTMS and APDMMS, initially undergo a fast hydrolysis to form silanols, followed by a condensation reaction with the surface hydroxyl groups as presented in Figure $2.7[60,91,173]$. The methods are convenient to use due to their simplicity and low setup cost. However, the quality of the formed layers are highly sensitive to the presence of water in the system. Polymerization of hydrolysed silane molecules can easily occur. In microfluidic systems, the liquid phase deposition is further limited by solvent compatibility with device materials.

Vapor-phase deposition. The vapor deposition method can be applied to surfaces by volatilization of organosilanes under heat or vacuum within a closed system $[91,108,209,224]$. The reaction mechanism is assumed to be similar to the aforementioned mechanism [69]. The approach has the advantage over liquidphase deposition that only a small amount of organosilane is used and that layers with higher homogeneity and quality can be routinely formed. The vapor-phase deposition of organosilanes is attractive for closed microfluidic systems, but its practical potential has not yet been fully explored. In this 
Table 2.1: Surface functionalization of silicon/glass-based microfluidic devices for protein immobilization.

\begin{tabular}{|c|c|c|c|}
\hline $\begin{array}{l}\text { Group } \\
\text { (on surface) }\end{array}$ & $\begin{array}{l}\text { Group } \\
\text { (to functionalize) }\end{array}$ & Step & Reagent \\
\hline \multirow[t]{5}{*}{ Hydroxyl } & Amine & $\mathrm{I}$ & $\begin{array}{l}\text { APTMS; APTES; APDMMS [40, } \\
69,108]\end{array}$ \\
\hline & Carboxylate & II & $\begin{array}{l}\text { Carboxyethylsilanetriol; } \\
\text { EDTA [108] }\end{array}$ \\
\hline & Epoxide & III & $\begin{array}{l}\text { GOPTMS; GOPTES; EPPTMS } \\
{[72,108,182,251,269]}\end{array}$ \\
\hline & Isocyanate & IV & ICPTES $[108,133]$ \\
\hline & Thiolate & $\mathrm{V}$ & MDS; MTS $[19,108]$ \\
\hline \multirow[t]{5}{*}{ Amine } & Disulfide & VI & $\begin{array}{l}\text { 2-Iminothiolane; SPDP }[85,108 \text {, } \\
\text { 190] }\end{array}$ \\
\hline & Aldehyde & VII & $\begin{array}{l}\text { Glutaraldehyde; Dextran } / \mathrm{NaIO}_{4} \\
{[108,122,133,269,274]}\end{array}$ \\
\hline & Maleimide & VIII & $\begin{array}{l}\text { S-SMCC; S-SMPB; BMH; EMCS } \\
{[85,133,222,269]}\end{array}$ \\
\hline & NHS-ester & IX & EDC/NHS $[108,133,269]$ \\
\hline & Carboxylate & $\mathrm{X}$ & $\begin{array}{l}\text { Glutaric anhydride; Citraconic an- } \\
\text { hydride; CM-dextran cis-Aconitic } \\
\text { anhydride }[85,108,246]\end{array}$ \\
\hline Carboxyl & NHS-ester & XI & NHS [108, 121,269] \\
\hline Epoxide & Carboxylate & XII & CM-dextran [72] \\
\hline Sulfhydryl & Disulfide & XIII & SPDP; TNB [108] \\
\hline
\end{tabular}

thesis, surface functionalization with aminosilanes using vapor-phase deposition is introduced and characterized in closed microfluidic devices.

Although many of the mentioned methods have been well established for open surface systems, they can still be generally improved. Moreover, the implementation of those methods for closed microfluidic devices is challenging due to the high risk of clogging and the susceptibility to cross-contamination of reagents. As such, protocols for reliable protein immobilization need to be tested and optimized for microfluidic systems, especially for the SMR devices.

Once the first layer of functional groups is introduced to the surfaces (Figure 2.6 and Table 2.1, (I)-(V)), protein immobilization is readily possible (Figure 2.6, (XVII) and (XIX)-(XXI)). Alternatively, subsequent functionalization can also 
modify the initially introduced functional groups and change the reactive groups with spacer arms or crosslinkers (Figure 2.6 and Table 2.1, (VI)-(XIII)). Proteins are then immobilized on the second functional layers of the surfaces (Figure 2.6, (XIV)-(XVI) and (XVIII)).

Using different functional groups, covalent coupling of proteins can be carried out by designing a site-specific reaction between functional groups of the proteins and the surfaces. The site-specific immobilization could allow reproducible immobilization and the binding sites can be designed in ways that do not affect the stability and functionality of the proteins. Depending on the commonly available protein functional groups located at N-, C-terminus or in the exposed side chains, the most commonly applied protein coupling techniques are classified into amine, carboxyl, thiol and hydroxyl couplings (Figure 2.6 and Table 2.1).

Amine coupling. The nucleophillic amine groups can react with surface epoxide groups, functionalized with e.g. GOPTMS, in a ring-opening process (XIX) $[108,133]$. Secondary amines are created under high $\mathrm{pH}$ conditions usually in the range of $\mathrm{pH}>9$. Alternatively, the interaction between protein amine groups and surface aldehyde groups leads to the formation of a labile Schiff's base that can be stabilized by reduction using sodium borohydride or sodium cyanoborohydride (XV). In this study, APTMS was used to introduce amino groups on the native glass/silicon surfaces (I) and glutaraldehyde was subsequently applied to generate surface aldehyde groups (VII) for amyloid seed fibril coupling. The amine groups of proteins can also react with functional isocyanate groups on the surfaces at alkaline $\mathrm{pH}$ values (XX). One drawback of the method is that unstable isocyanate compounds can be decomposed rapidly by moisture. Another common immobilization reaction is the protein amine coupling to NHS-ester activated surfaces (XVIII). The NHS-esters on the amine-modified surfaces can either be generated by applying bifunctional NHS-esters (IX) (e.g. DSS and S-SMCC) or by partially activating the carboxyl groups of the coupling agents (e.g. CM-dextran in this study) (X) with EDC/NHS , followed by another EDC/NHS-activation-step to enrich the surface carboxyl groups (XI). The reaction creates stable amide linkage rapidly under physiological pH conditions, but hydrolysis of the NHS-esters should be taken into account in practice. Amine coupling can be applied to link nearly all protein molecules with amine groups at their N-terminus. Some proteins also contain lysine residues in the side chains.

Carboxyl coupling. Functional carboxyl groups exist at the C-terminus of a pro- 
tein and in aspartic acid and glutamic acid residues which can be present at protein surfaces. The protein coupling to the amine-modified surfaces requires an additional step to activate the carboxyl groups due to their low nucleophilicity. CDI can be used to activate the carboxyl groups of proteins for further coupling (XVII). The optimal conditions include alkaline solutions in the range of pH 7-9. Alternatively, EDC/NHS can also efficiently activate the carboxyl groups of proteins. However, care must be taken when using these methods, as proteins also contain amine groups in themselves and a self cross-linking could occur in the solutions.

Thiol coupling. Reactive groups that are able to react with protein sulfhydryl groups are the second most common groups. As illustrated in Figure 2.6, direct formation of disulfide bonds takes place between sulfhydryl-containing proteins and surfaces (XXI). Alternatively, thiol-disulfide exchange reactions are used to couple sulfhydryl-containing proteins to surface disulfide groups (XIV). An important feature of this process is that the bonds are reversible using disulfide reducing agents such as DTT or TCEP, allowing regeneration of the surface functional groups. Alternatively, maleimides are favorable functional groups that are contained in many heterobifunctional crosslinking agents. The alkylation reaction of protein sulfhydryl groups with the double bond of surface maleimide groups undergoes efficiently at pH 6.5-7.5 (XVI). However, hydrolysis of the maleimide groups in form of a ring-opening reaction may occur before and even after the sulfhydryl coupling. Similar to protein amine coupling, reactive sulfhydryl can react with surface epoxy groups (XIX). The required $\mathrm{pH}$ condition is closer to the physiological range of 7.5-8.5 [108]. The reaction of sulfhydryl groups with NHS-esters does not yield stable conjugates and is therefore not applicable.

Hydroxyl coupling. Similar to the coupling of amine and sulfhydryl groups to epoxy-modified surfaces, hydroxyl groups of serine and threonine residues contained in proteins can react with the surface epoxy groups in a ring-opening process. The reaction with hydroxyls (XIX) requires high alkaline conditions (pH 11-12). Hydroxyl groups can also bind to isocyanate groups on the substrate surfaces $(\mathrm{XX})$.

\subsubsection{Physisorption}

Physisorption (or physical adsorption) is frequently employed in protein immobilization [132,221]. By physisorption, proteins adsorb to silicon dioxide surfaces via intermolecular forces such as electrostatic, hydrophobic, van der Waals or hydrogen 
bonding interactions. Compared to covalent coupling methods that usually require multistep operations and long incubation periods, physisorption can normally be performed quickly and conveniently, which makes it a preferred method for protein immobilization in early, proof-of-principle experiments. However, several drawbacks limit the application of the method: (1) physically adsorbed proteins are not as stable as proteins immobilized by covalent or bioaffinity binding; (2) the immobilized protein layer is randomly oriented; (3) it is challenging to generate reproducible physisorption since the intermolecular forces highly depend on environmental conditions such as $\mathrm{pH}$, ionic strength and temperature; (4) the density of adsorbed molecules is generally difficult to evaluate; and (5) the prevention of non-specific binding is difficult.

\section{Electrostatic Functionalization}

In protein chemistry, functional groups of protonated amine $\left(-\mathrm{N} H_{3}^{+}\right)$and quaternary ammonium cations $\left(-\mathrm{N} R_{3}^{+}\right)$contribute to the positive charge of proteins and functional groups of carboxylic acid $\left(-\mathrm{COO}^{-}\right)$and sulfide $\left(-S^{-}\right)$contribute to the negative charge. The overall net charge is related to the electrostatic interaction between the protein and a surface. The proteins used in this thesis ( $\alpha \mathrm{S}$, insulin) to study amyloid formation are negatively charged in physiological solutions, with $\mathrm{pI}$ values lower than $p H=7$. In contrast, silicon dioxide surfaces are negatively charged with deprotonated hydroxyl groups when the $\mathrm{pH}$ value of the solution is greater than $\mathrm{pH}$ 4. Poly-L-lysine (PLL) is frequently used to generate positive surface charge and to link the negatively charged proteins. In this study, PLL has been employed to immobilize $\alpha \mathrm{S}$ seed fibrils on the walls of the SMR channels. In case that positively charged proteins are desired to be immobilized, generation of an additional surface layer with negative charge, e.g. by use of polyacrylamide (PA) gels, is an alternative to direct binding of proteins to the surface. Compared to the direct interaction on the $\mathrm{SiO}_{2}$ surface, the interaction can be enhanced by generating more negative charge. The electrostatic interaction is generally stronger than other physisorption interactions.

\section{Functionalization Based on Intermolecular Forces}

The hydrophobic interaction, hydrogen bonding and Van-der-Waals interaction are common adhesion forces that can be used to functionalize solid surfaces by physisorption [133]. Protein immobilization using hydrophobic interactions has been frequently applied on many microfluidic devices made of PDMS, PMMA 
and other polymeric hydrophobic materials. The simplicity makes it a preferred immobilization method in early, proof-of-concept experiments.

\subsubsection{Bioaffinity Interaction}

The bioaffinity interactions have advantages of specificity and binding orientation. In addition, the bioaffinity interactions can be significantly stronger than electrostatic interaction and can sometimes be regenerated with chemical treatment, $\mathrm{pH}$ change or heat treatment [133]. Combined with either physisorption or covalent coupling techniques, bioaffinity reagents are mostly used as intermediate binding molecules between the surface and proteins $[108,156]$.

One of the most widely employed bioaffinity interaction candidates is avidinbiotin. Avidin or its derivatives (e.g. streptavidin, neutravidin or nitrividin) can be physisorbed or covalently bound to the surface. Regardless of $\mathrm{pH}$, temperature or denaturing agents, biotinylated proteins can bind to the avidin rapidly. The limitation of the method is that additional biotinylation is required for proteins.

Beside the avidin-biotin binding, several other protein immobilization methods using affinity interaction exist [222]: (1) a single-strand DNA (ssDNA) can be attached to the protein as a tag and can further bind to the pre-immobilized complementary DNA on the surfaces via specific hybridization; (2) protein A and protein $\mathrm{G}$, which are extracted from bacteria, can be used to bind specifically to antibodies ( $\operatorname{IgG}) ;(3)$ immobilized antibodies are able to capture the targeted antigen epitope and (4) polyhistidine tags of proteins interact specifically with metal ions $\left(\mathrm{Ni}^{2+}, \mathrm{Cu}^{2+}, \mathrm{Zn}^{2+}\right.$ etc. $)$.

\subsubsection{Combination of Immobilization Techniques}

Covalent coupling, physisorption and bioaffinity interactions can be combined to address the limitations of each method and to achieve a better control of protein immobilization. The most common combinations include physisorption-covalent coupling [273], covalent-bioaffinity immobilization [75, 149, 244], physisorptionbioaffinity immobilization $[34,68,161,226]$ and covalent-physisorption-bioaffinity immobilization $[67,234,275]$. In addition to all the common chemical agents that are used to perform the surface functionalization and protein immobilization, natural adhesives have recently been reported to be able to functionalize sur- 
faces $[150,162,185,257]$. Polydopamine that is secreted by mussels contains plentiful catechol and amine functional groups and forms strong covalent and hydrophobic interactions with virtually all types of inorganic and organic surfaces, including silicon oxide, polymers and even noble metals. Polydopamine-modified surfaces serve as a multifunctional platform for further protein coupling and interaction. In microfluidic systems, the evaluation criteria for an optimal combination are sufficient density, retained biological function of the immobilized molecules, low non-specific binding and stability in the presence of continuous flow conditions.

In this study, electrostatic and covalent immobilization techniques have also been combined to immobilize amyloid seed fibrils. PLL-grafted-carboxymethyl(CM)dextran binds to the $\mathrm{SiO}_{2}$-surfaces via the interaction of positively charged lysine residues with the negatively charged surfaces. Furthermore, the carboxyl groups can be activated using EDC/NHS solutions and the seed fibrils can thus be covalently bound to the dextran matrix on the surface. These immobilization techniques have been assessed with regards to stability and non-specific adsorption, and the results are presented in Section 5.1.2. 

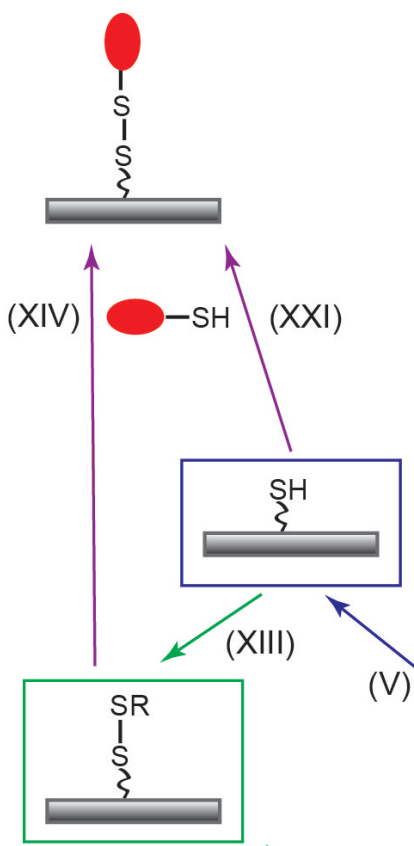

(V)
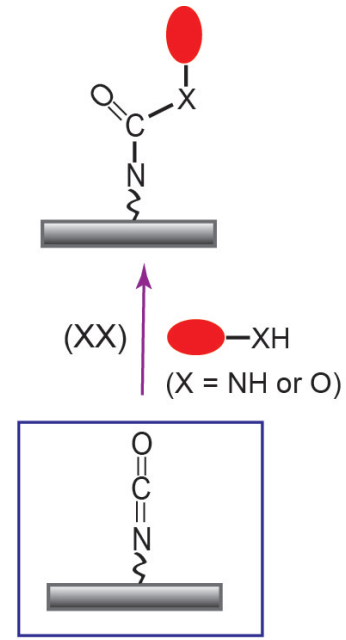

(IV)
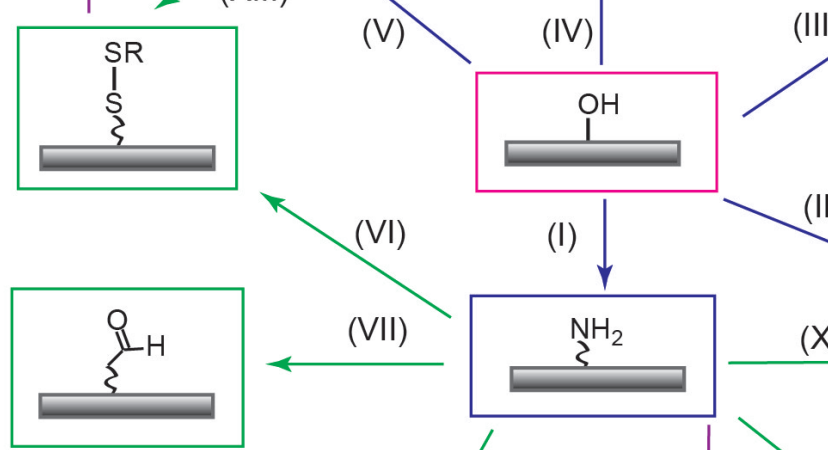

(VI)

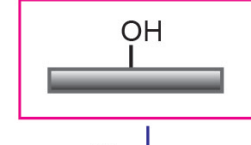

(I)

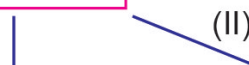

(II)
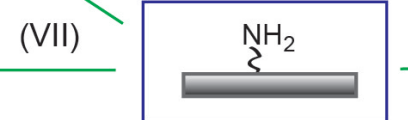

$(\mathrm{X})$

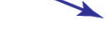

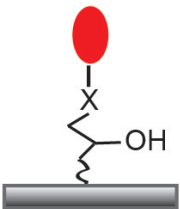

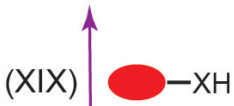

$(\mathrm{X}=\mathrm{NH}, \mathrm{O}$ or $\mathrm{S})$

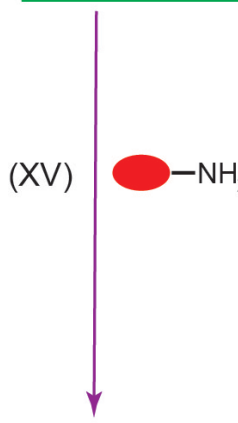

(VIII)
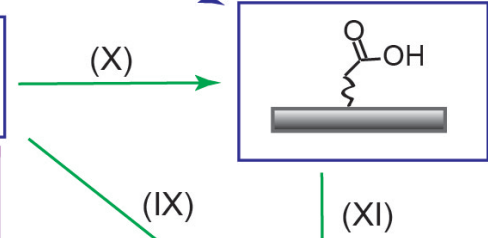

$(\mathrm{XI})$

(XII)
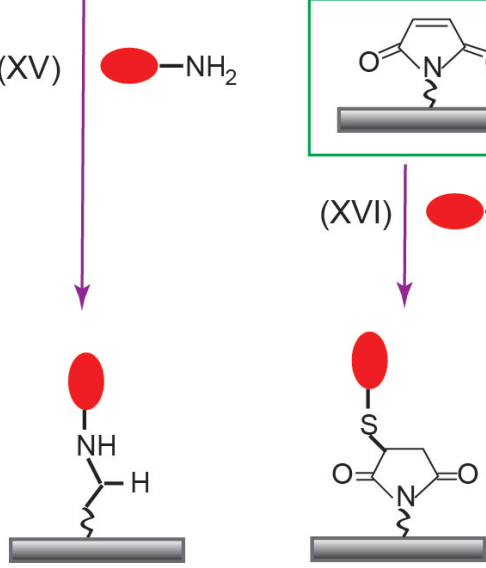

(XVII)
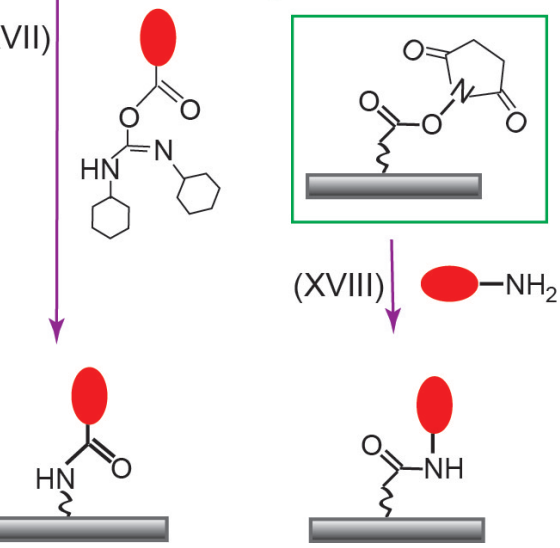

Figure 2.6: Covalent immobilization of proteins to silicon and glass surfaces with different functional groups. The reagents used for steps (I)-(XIII) are listed in Table 2.1. The sitespecific protein couplings with surface functional groups correspond respectively to steps (XIV)-(XXI). 


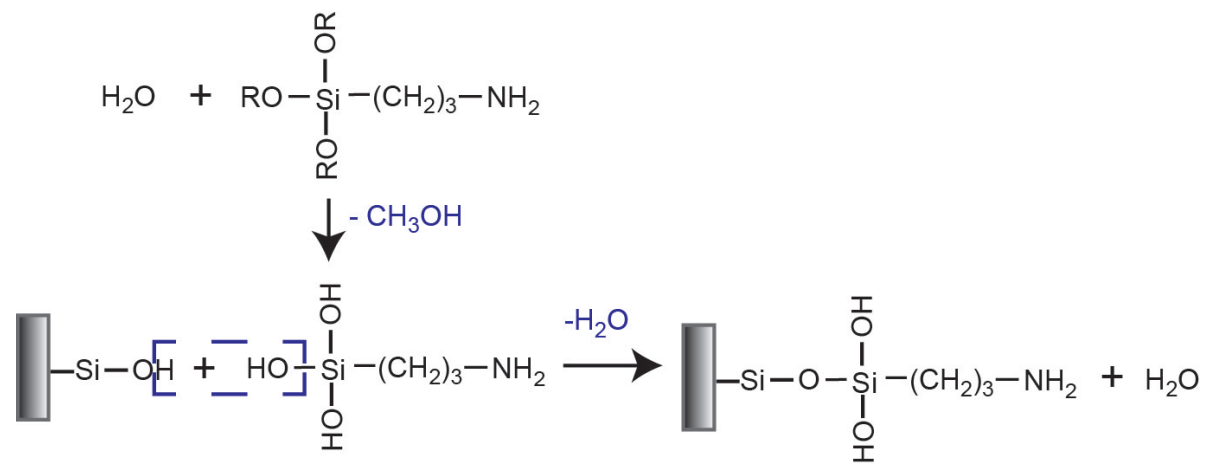

Figure 2.7: Mechanisms of covalent coupling of alkoxysilanes with surface hydroxyl groups. The RO- group here stands for any alkoxy group (in this study: the methoxy groups of APTMS and APDMMS). 


\subsection{Surface Passivation}

Surface passivation is necessary to block the remaining functional groups after protein immobilization and to prevent non-specific molecule adsorption. In surfacebased SMR measurements or other biosensor techniques, low non-specific protein adsorption contributes to low background signal. In this thesis, sufficient surface passivation of protein monomer molecules ensures the reliability and precision of amyloid kinetic measurements. As a subsequent step after surface functionalization and protein immobilization, the passivation can be carried out by physisorption or covalent binding.

\section{Physisorption}

Albumin-based agents, e.g. BSA, can bind to the glass/silicon surfaces as a result of hydrophobic interaction, preventing further adsorption of proteins to the surfaces. Electrostatically, hydrophillic dextran or PLL-grafted PEG agents are widely used to prevent non-specific binding of proteins. PLL-grafted-CM-dextran has been deposited on the SMR channel surfaces to suppress non-specific adsorption of $\alpha \mathrm{S}$ fibrils. Furthermore, the direct use of zwitterionic polymers, such as poly(carboxybetaine), has been reported to efficiently suppress the non-specific protein adsorption on hydrophobic surfaces (e.g. PDMS) while providing specific functional groups for protein immobilization [126]. More recently, lipid bilayer membranes have been developed to modify the inner walls of microfluidic channels since hydrophobic interaction and Van-der-Waals force enables lipid deposition on the surfaces. The lipid bilayers are able to provide better passivation than albumin-based agents [117,207].

\section{Covalent passivation}

To avoid proteins interfering with the subsequent reactions or application, passivation of the functional groups in excess after the specific protein immobilization is of great importance. The passivation molecule is usually a small compound containing a functional group able to couple with the surface functional group at one end and a group at the other end that does not interact with proteins. The passivation compounds are selected depending on the surface functional groups, and the reactions are similar to those of protein coupling with surface functional groups described in the covalent coupling section (2.2.1). 
Passivation of surface amine groups. Several acylation reagents, such as sulfoNHS acetate and acetic anhydride, have been reported to effectively passivate the primary amine groups [108].

Passivation of surface sulfhydryl groups. Partis et al. used maleimide reactions to form thioether linkages and specifically block the reactive sulfhydryl groups in pH 6.5-7.5 [201]. N-Ethyl maleimide is a permanent blocking agent. In contrast, reversible passivation can be performed using Ellman's Reagent or other disulfide agents to form disulfide bonds, which can be cleaved by addition of reducing agents such as DTT.

Passivation of surface aldehyde groups. Amine-containing molecules, such as ethanolamine, Tris and hydrazides, can be applied to block the reactive aldehyde groups on the surface by forming secondary amine linkages in reductive amination procedures.

Passivation of surface carboxylate groups. Similar to passivation of surface aldehyde groups, amine-containing molecules can also be applied to passivate the carboxylate groups. However, additional EDC molecules are required in the precedure to activate the carboxylate groups for the coupling. 


\section{Chapter 3}

\section{Materials and Methods}

\subsection{Chemicals and Materials}

All chemicals that were used for buffer preparation, cleaning, surface modification, surface characterization, and kinetic measurements (elongation rate determination, dissociation monitoring, inhibitor screening) are listed in Table 3.1.

\section{$3.2 \quad$ Facility}

Important facilities used in the current thesis for the sample and manifold preparation, fluorescence detection, fluid delivery and detection systems of the transparent microchannels and the SMR devices are listed in Table 3.2. 
Table 3.1: Chemicals for the experiments.

\begin{tabular}{|c|c|c|}
\hline Application & Chemicals & Company \\
\hline \multirow[t]{2}{*}{ Cleaning } & $\mathrm{H}_{2} \mathrm{SO}_{4}(98 \%)$, ethanol, $\mathrm{NaOH}(1 \mathrm{M})$ & Merck, Germany \\
\hline & $\mathrm{H}_{2} \mathrm{O}_{2}$ & Fisher Scientific, Germany \\
\hline \multirow[t]{2}{*}{ Buffer } & HEPES, PBS(10x conc.) & Carl Roth, Germany \\
\hline & Glycine & \\
\hline \multirow[t]{3}{*}{ Silanization } & APTMS, APDMMS & Sigma-Aldrich, Germany \\
\hline & AF555-NHS & Invitrogen, Germany \\
\hline & NHS-biotin & Pierce Biotechnology, USA \\
\hline \multirow[t]{9}{*}{ Immobilization } & PLL, Human insulin, PLGA, NaCN & Sigma-Aldrich, Germany \\
\hline & Glutaraldehyde, $\mathrm{NaCNBH}_{3}, \mathrm{NaN}_{3}, \mathrm{NaCl}$ & Carl Roth, Germany \\
\hline & EZ-link Sulfo-NHS-LC-biotin & Thermo Scientific, Germany \\
\hline & rAcGFP1 & Clontech, Germany \\
\hline & PLL-grafted-CM-dextran & SuSoS, Switzerland \\
\hline & CM-dextran (70 kDa) & TdB-Consultancy, Sweden \\
\hline & $\alpha \mathrm{S}$ & MPIBPC (Prof. Zweckstetter) \\
\hline & & Germany \\
\hline & NDA, AF555-NHS-ester & Invitrogen, Germany \\
\hline \multirow[t]{5}{*}{ Kinetics } & Ectoine, Trehalose & Carl Roth, Germany \\
\hline & $\mathrm{CaCl}_{2}, \mathrm{MgCl}_{2}$ & $\mathrm{ZnCl}_{2}$ Carl Roth, Germany \\
\hline & Methylene blue & Roth, Germany \\
\hline & PcTS & MPIBPC (Prof. Zweckstetter) \\
\hline & Microbead NIST $(1.5 \mu \mathrm{m})$ & Polysciences, USA \\
\hline
\end{tabular}


Table 3.2: Important facilities used in the experiment.

\begin{tabular}{|c|c|}
\hline Equipment & Company \\
\hline TKA MicroPure Ultra Pure Water System & $\begin{array}{l}\text { Thermo Fisher Scientific, Ger- } \\
\text { many }\end{array}$ \\
\hline \multicolumn{2}{|l|}{ NanoDrop 3300 florospectrometer } \\
\hline NanoDrop 2000c UV-VIS spectrometer & \\
\hline FEMTO Surface Plasma Cleaner & Diener Electronic, Germany \\
\hline Inverted Axio Observer D1m Microscope & Zeiss, Germany \\
\hline \multicolumn{2}{|l|}{ Filter set 44 and $75 \mathrm{HE}$} \\
\hline \multicolumn{2}{|l|}{ Objective $5 \mathrm{x}, 10 \mathrm{x}, 20 \mathrm{x}$, etc. } \\
\hline ULTRASONIC cleaner & VWR, Germany \\
\hline Centrifuge 5430R & Eppendorf, Germany \\
\hline Universal oven & Mommert, Germany \\
\hline VERSALASER & Universal Laser System, USA \\
\hline LabRoutine PH-meter & $\begin{array}{l}\text { METTLER TOLEDO, Switzer- } \\
\text { land }\end{array}$ \\
\hline ABT 1205 DM balance & KERN, Germany \\
\hline Incubator & AQUA $^{\mathbb{B}}$ LYTIC \\
\hline Pressure regulator and manometer & $\begin{array}{l}\text { RV Regler \&Verfahrenstechnik, } \\
\text { Germany }\end{array}$ \\
\hline Electrical pressure regulators and valves & SMC Pneumatik, Germany \\
\hline Autosampler & Agilent, USA \\
\hline $300 \mathrm{MHz}$ Synthesized function generator & Stanford Research Systems, USA \\
\hline Tektronix TDS 2004B Oszilloscope & Farnell, Germany \\
\hline TED 200c Temperature controller & Thorlabs \\
\hline \multicolumn{2}{|l|}{ Other optical components } \\
\hline Laboratory DC power supply GPS-2303 & GWINSTEK, USA \\
\hline NI-DAQ \& Labview & National Instrument, USA \\
\hline CCD Camara & Andor Technology, Ireland \\
\hline Bode analyzer & Omicron LAB, USA \\
\hline SMZ-168 Stereo Zoom Microscope & Motic, Germany \\
\hline
\end{tabular}




\subsection{Sample Preparation}

\subsubsection{Fabrication of SMR Devices}

The applied SMR devices, torsional microresonators with embedded $3 \times 8 \mu \mathrm{m}$ microchannels, were fabricated on six inch silicon wafers at Innovative Micro Tech-nology (Santa Barbara, CA) and obtained from the laboratory of Prof. Scott Manalis (MIT, Cambridge MA) [34]. The fabrication process was described in Reference [34]. The fluid interconnects to the SMR chip were made using a Teflon manifold.

\subsubsection{Fabrication of Transparent Microchannels}

Transparent microfluidic devices with microchannels that are similar in design to SMR microchannels were fabricated by H. Feindt using conventional microfabrication techniques at the Center of Advanced European Studies and Research (caesar, Bonn, Germany). Devices with 75 x $66 \mu \mathrm{m}$ microchannels were fabricated on four inch silicon wafers using lithographic patterning, deep reactive-ion etching (DRIE) on both sides and anodic bonding to a plain borofloat wafer. Devices with $0.7 \times 2 \mu \mathrm{m}$ nanochannels were fabricated similarly by first patterning the shallow channels followed by patterning the deep bypass channels. The fabrication and packaging process of such nanochannel devices is depicted in Figure 3.1.

\subsubsection{Preparation of Insulin Fibrils}

\section{Preparation of monomer solutions}

Inuslin monomers were freshly dissolved in $1 \mathrm{~mL}$ of either HEPES $(10 \mathrm{mM})$ or glycine buffer $(50 \mathrm{mM})$ at $\mathrm{pH} 2$ with concentrations ranging from $1-6 \mathrm{mg} / \mathrm{mL}$ for further fibril formation. For concentration-dependent elongation measurements, the insulin monomers were prepared with concentrations of $0.06,0.1,0.2,0.4,0.6,0.8,1$, 2,4 , and $6 \mathrm{mg} / \mathrm{mL}$. All the concentrations were determined by UV-VIS spectroscopy at $280 \mathrm{~nm}$ absorption wavelength. The monomer solutions were centrifuged at $30000 \mathrm{~g}$ for 30 minutes at $4^{\circ} \mathrm{C}$ and only the supernatant of the solutions was carefully removed with pipettes for further use. In the concentration-dependency measurements, the monomer insulin solutions were kept at $4^{\circ} \mathrm{C}$ until they were used for the real-time measurements. 

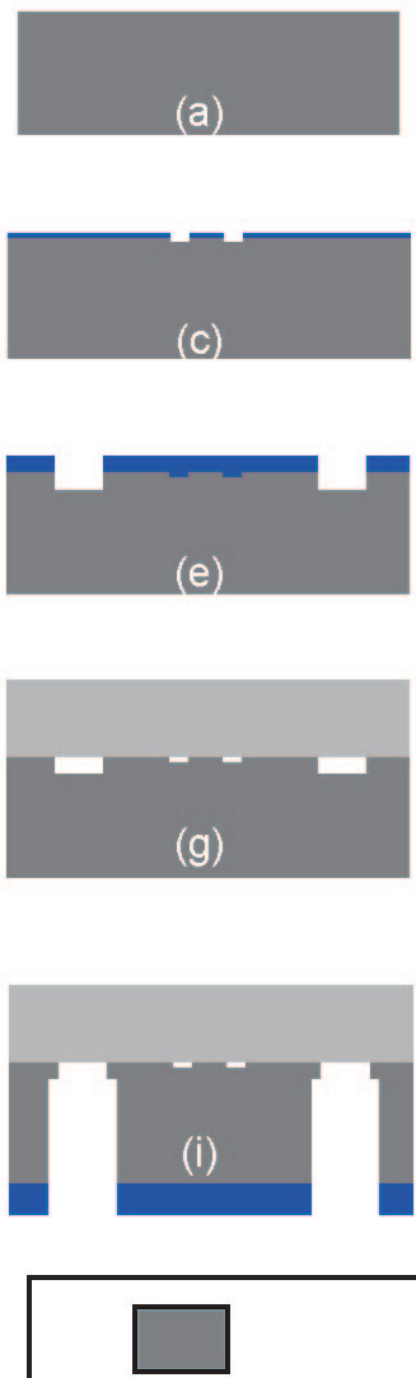

Si
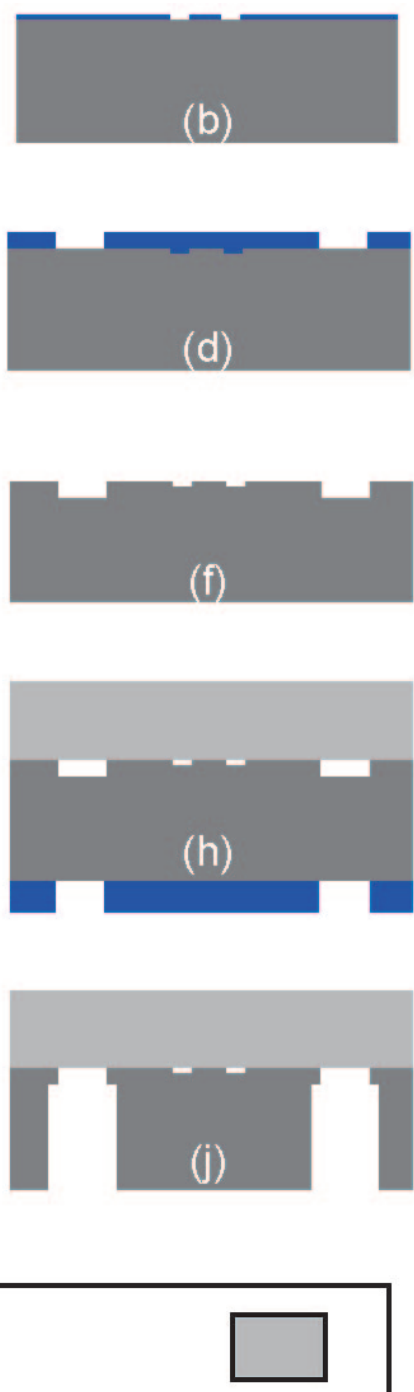

Glass

Figure 3.1: Fabrication and packaging process of transparent nanochannels (700 nm x $2 \mu \mathrm{m}$ ): (a) 4" silicon wafer (300 $\mu \mathrm{m}$ thick, $<100>$ orientation); (b) Patterning nanochannels using photolithography; (c) RIE; (d) Patterning deep bypass channels using photolithography; (e) DRIE; (f) Resist strip and cleaning with piranha solution; (g) Anodic bonding with borofloat glass; (h) Patterning inlet and outlet holes using photolithography on back side; (i) DRIE; (j) Dry resist strip using oxygen plasma.

\section{Preparation of long insulin fibrils}

The fresh monomer solutions were incubated at $37^{\circ} \mathrm{C}$ in $1.5 \mathrm{~mL}$ borosilicate glass vials with rubber-lined closures. To standardize the measurements, $6 \mathrm{mg} / \mathrm{mL}$ monomer solutions were used to start long fibril formation. The solutions were stirred with micro stirring bars $(3 \times 7 \mathrm{~mm})$. At two hour intervals, the vials were gently shaken to distribute fibrils evenly in the vials before withdrawing $3 \mu \mathrm{L}$ aliquots to measure the fluorescence by binding with ThT. The fibril formation was complete 
when the fluorescence intensity did not increase.

The long fibrils were also prepared by seeding short fibril fragments with the same starting concentrations in monomer solutions. The solutions were incubated similarly and the fibril formation ended within 2-4 hours. The solutions were stored at room temperature.

\section{Preparation of short insulin fibrils}

Short insulin fibrils were an important basis as seeds for the quantitative kinetic measurements. To obtain short insulin fibrils of uniform size, the solutions with long insulin fibrils (starting concentration of $6 \mathrm{mg} / \mathrm{mL}$ ) in HEPES buffer (10 mM, pH 2.0) were diluted by a factor of six and ultrasonicated for $2.5 \mathrm{~h}$ with 15 minutes intervals at $4^{\circ} \mathrm{C}$. The ultrasonicated samples were quickly transferred into $0.2 \mu \mathrm{m}$ Millipore-filter vials and filtered at $12000 \mathrm{~g}$ at room temperature.

\subsubsection{Preparation of $\alpha \mathrm{S}$ Fibrils}

The samples of $w t-\alpha \mathrm{S}$ fibrils and monomers were obtained from the Department of NMR-based Structural Biology of the Max Planck Institute of Biophysical Chemistry, Göttingen, Germany. The stock $\alpha \mathrm{S}$ fibril solutions (starting concentration of $20 \mu \mathrm{M}$, ca. $0.3 \mathrm{mg} / \mathrm{mL}$ ) were kept at room temperature. Prior to use, the sample solutions were treated with ultrasonication for 10 minutes at room temperature.

\subsubsection{Surface Functionalization with Silane Coupling Agents}

\section{Vapor phase deposition of aminosilanes}

Microfluidic channels were silanized using 3-aminopropyl trimethoxysilane (APTMS) by vapor phase deposition as shown in Figure 3.2. Firstly, the microchannel devices were treated with atmospheric plasma at a pressure of $0.4-0.6$ mbar for 10 minutes to clean the external surfaces. APTMS $(3 \mu \mathrm{l})$ was then transferred into $10 \mathrm{~mL}$ vials in an argon filled glove bag to avoid trace amount of water. The capped glass vials were then incubated at $40-80^{\circ} \mathrm{C}$ for $6-16 \mathrm{~h}$. To maintain an unsilanized reference channel, one channel was protected with Kapton ${ }^{\circledR}$ tape. All tape was removed prior to rinsing with ethanol. Afterwards, the devices were removed from the glass vials and connected to a pressure controlled fluidic manifold. All channels were 
then rinsed thoroughly with ethanol and dried with nitrogen gas.

(a) Silanization

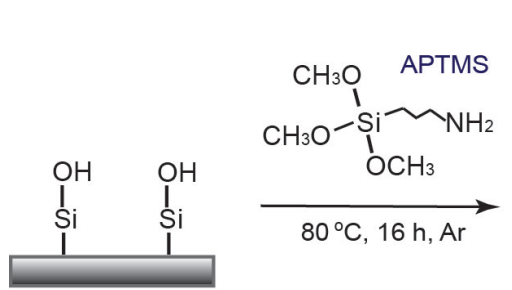

(b) Labeling

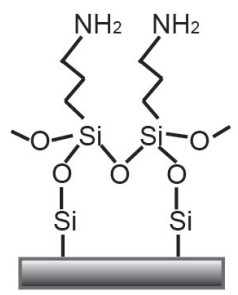

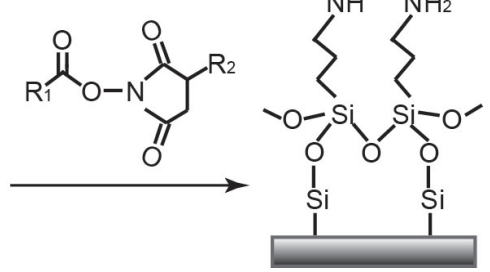

Figure 3.2: Reaction schematic for covalent attachment of NHS-based molecules to silicon and glass substrates. (a) Vapor phase deposition of APTMS; (b) Labeling the reactive amine functional groups with AF-NHS-ester $\left(R_{1}=\right.$ Alexa Flour $\left.{ }^{\circledR} 555 ; R_{2}=\mathrm{H}\right)$ and $/$ or sulfo-NHS-biotin $\left(R_{1}=\right.$ LC-biotin; $\left.R_{2}=-S O_{3}^{-}\right)$.

Vapor phase silanization was also performed on borofloat glass slides (ca. $9 \times 12 \mathrm{~mm})$. The slides were initially cleaned in piranha solution for a minimum of 30 minutes. The slides were then thoroughly rinsed with pure water, dried under nitrogen gas, and plasma treated for 10 minutes. To maintain an area of the slides unsilanized, one half on both sides of the slide was covered with Kapton ${ }^{\circledR}$ tape.

\section{Liquid phase deposition of aminosilane}

To perform the liquid deposition, APTMS was dissolved in toluene at a concentration of $5 \%$ and also in ethanol with a concentration of $2 \%(\mathrm{v} / \mathrm{v})$.

Using toluene as the solvent, the solution was transferred into the transparent microchannels using chemically resistant FEP tubing. The surfaces were incubated with the APTMS toluene solution for $20 \mathrm{~h}$ at room temperature and then rinsed with toluene, isopropanol, and water for at least 10 minutes respectively.

The APTMS in ethanol was introduced to the microchannel surfaces, incubated for $2 \mathrm{~h}$ at room temperature and then rinsed with ethanol and water.

\subsubsection{Protein Immobilization and Passivation}

\subsubsection{Covalent Coupling of Insulin Fibrils}

Short insulin fibril samples obtained as described in Section 3.3.3 were immobilized as illustrated in Figure 3.3 following the same procedure in transparent microchannels and SMR devices. The flow direction inside the small microchannels was controlled 
by changing the pressure differences at inlet and outlet of both connected bypass channels. The immobilization process is detailed as follows:

(I) Functionalization with amine groups. The in-channel functionalization was done by vapor phase deposition of APTMS as described in Section 3.3.5. The microchannels were then rinsed with ethanol and subsequently with water. In addition, the microfluidic devices were heated and maintained at $37^{\circ} \mathrm{C}$ for further coupling and immobilization.

(II) Functionalization with aldehyde groups. To introduce reactive aldehyde groups, glutaraldehyde solution ( $50 \%$ in water) was diluted by a factor of five times and centrifuged at $30000 \mathrm{~g}$ for 15 minutes at $4^{\circ} \mathrm{C}$ to reduce the content of large glutaraldehyde aggregates in solution. The supernatant was carefully removed and introduced into the bypass channels and the small microchannels for 5 minutes followed by replacing with water. The reducing agent, $\mathrm{NaCNBH}_{3}$, was dissolved in water at a concentration of $10 \mathrm{mg} / \mathrm{mL}$ and introduced into all the channels for 10 minutes to stabilize the immobilized aldehyde groups on the surfaces.

(III) Immobilization of insulin seed fibrils. The solutions inside all microchannels were replaced with HEPES buffer (10 mM, pH 2.0). The solutions with short fibrils obtained as described in Section 3.3.3 were introduced to one of both bypass channels and the small microchannel and reacted with the functionalized surfaces for 15 minutes while the other bypass channel was filled with the HEPES buffer. The insulin seeds were then replaced with HEPES buffer, and all the channels were changed back to water followed by stabilizing the proteins on the surface with $10 \mathrm{mg} / \mathrm{mL} \mathrm{NaCNBH}_{3}$ solution for 20 minutes.

(IV) Passivation of the reactive surfaces. The flow direction was changed from the bypass channel without immobilized seed fibrils (the left channel in Figure 3.3) to the one with immobilized seeds (the right channel). The glycine buffer (50 mM, pH 2.0) was used to passivate the remaining aldehyde groups in the left bypass channel and the small microchannel for 10 minutes, followed by $\mathrm{NaCNBH}_{3}$-reduction for 20 minutes and then filled with water.

(V) Insulin elongation measurements. The channels were all filled with the buffer solutions and inuslin monomer solutions were then introduced to the small microchannel from the passivated site.

(VI) Surface cleaning and regeneration. To reuse the same microfluidic devices, pirahna solutions were applied for 10 minutes to clean all the channel 
surfaces followed by rinsing with water. The native surfaces with hydroxyl groups were thereby regenerated and ready again for surface functionalization with aminosilanes.

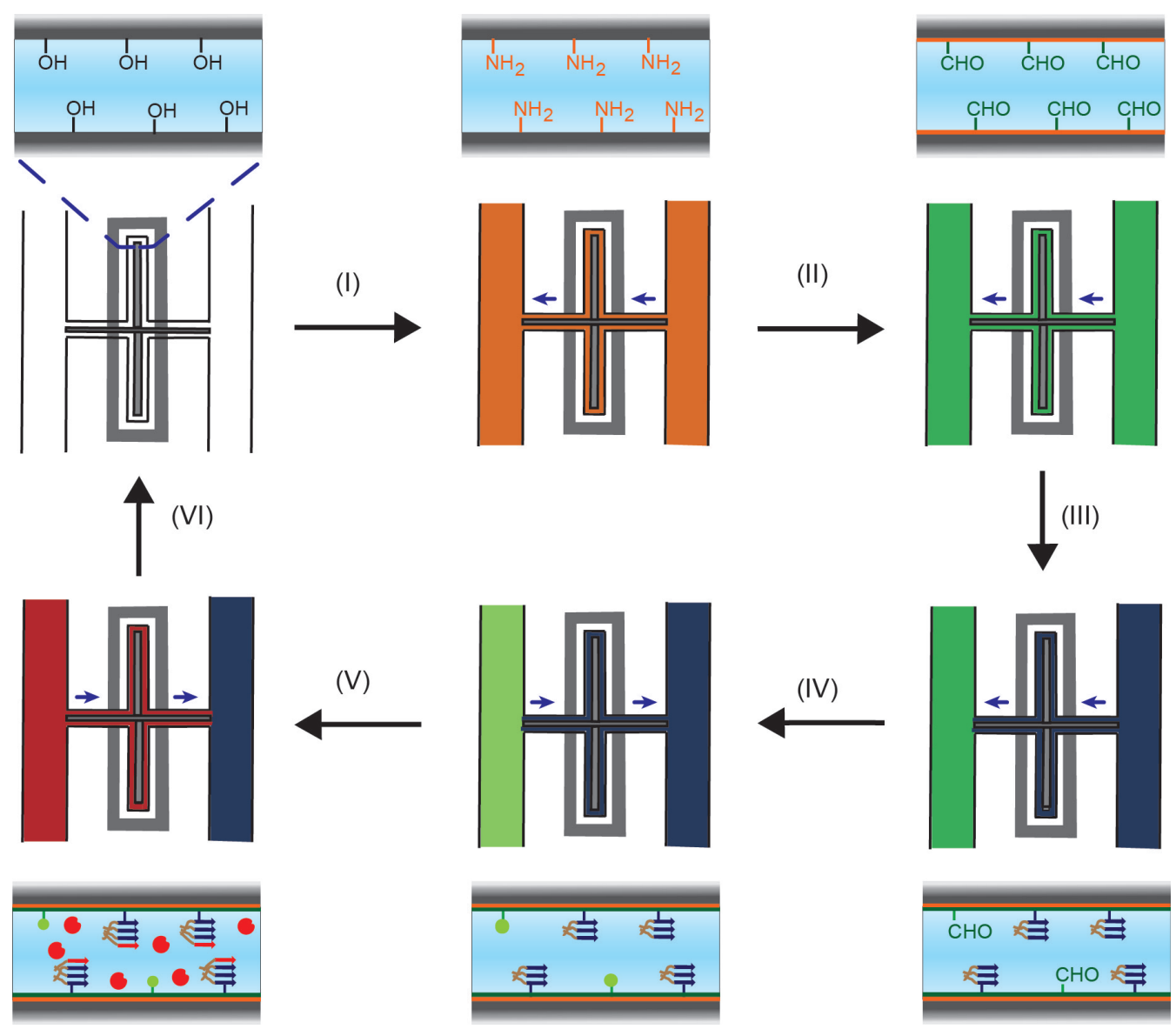

Figure 3.3: The covalent immobilization of seed insulin fibrils for elongation detection via (I) functionalization with amine groups; (II) functionalization with aldehyde groups; (III) immobilization with insulin seed fibrils; (IV) passivation of the reactive surfaces; (V) insulin elongation measurements and (VI) surface cleaning and regeneration.

\subsubsection{Electrostatic Adsorption of $\alpha$ Synuclein}

Poly-L-lysine and PLL-grafted-CM-dextran were used to immobilize $\alpha \mathrm{S}$ seed fibrils in the early elongation experiments. $\alpha \mathrm{S}$ fibrils were ultrasonicated for 10 minutes at room temperature to obtain short seed fibrils in fragments.

\section{Fibril immobilization using PLL}

Initially, the microchannels were filled with water. PLL solutions $(0.1 \mathrm{mg} / \mathrm{mL}$, 70-150 kDa) were introduced to the microchannel surfaces for 10 minutes and 
replaced with water and subsequently HEPES buffer at $\mathrm{pH}$ 6.5. Seed $\alpha \mathrm{S}$ fibrils were injected into one of the two bypass channels and the small microchannel for 15 minutes. After rinsing with buffer solution, monomer solutions with various concentrations were introduced to start fibril elongation.

\section{Fibril immobilization using PLL-grafted-CM-dextran}

$\alpha \mathrm{S}$ seed fibrils were also immobilized using PLL-grafted-CM-dextran, which was dissolved in water at a concentration of $1 \mathrm{mg} / \mathrm{mL}$ and mixed with an aqueous solution of $0.8 \mathrm{M}$ EDC and $0.2 \mathrm{M} \mathrm{NHS}$ at the volume ratio of $1: 1$. The mixture was injected into the microchannels for 5-10 minutes to generate functional carboxyl groups on the surface and then replaced by water and HEPES buffer (50 mM, $100 \mathrm{mM} \mathrm{NaCl}, \mathrm{pH}$ 6.5). The surface functional groups were then further activated with the aqueous EDC/NHS-solution for one minute, followed by the injection of $\alpha \mathrm{S}$ fibril solutions for 15 minutes.

\subsubsection{Microchannel Surface Regeneration}

The surfaces of inner walls inside the transparent microchannels and SMR devices were cleaned and regenerated with piranha solution (2:1 sulfuric acid/hydrogen peroxide).

Alternatively, alkaline $\mathrm{NaOH}$ solutions $(0.1 \mathrm{M})$ were also used to regenerate the native surfaces with hydroxyl groups for one hour. This method was applied in transparent microchannels. In SMR devices, the $\mathrm{NaOH}$-cleaning was inappropriate since $\mathrm{NaOH}$ can thin the silicon walls of the suspended microchannel, enhancing the risk of leaking into the vacuum cavity surrounding the resonator.

\subsection{Biophysical Detection Techniques}

\subsubsection{Fluorescence Quantification of Amine Groups}

\subsubsection{Fluorescence Labeling}

\section{Labeling with fluorescent NHS-esters}

Fluorescence labeling of amine modified surfaces was performed using AF555-NHS alone ( 5 and $10 \mu \mathrm{M})$ or with a mixture of AF555-NHS-ester and sulfo-NHS-biotin as 
shown in Figure 3.2. Mixtures were prepared from the solutions of AF555-NHS-ester $(70-350 \mu \mathrm{M})$ and sulfo-NHS-biotin $(5-5.5 \mathrm{mM})$ in $10 \mathrm{mM}$ HEPES buffer at $\mathrm{pH} 8$. The total solution concentration of NHS esters was kept constant at $5 \mathrm{mM}$ for all the mixtures while varying the stoichiometry of AF555-NHS-ester and sulfo-NHS-biotin. The resulting AF555-NHS-ester concentration in each solution mixture ranged from $1.25 \mu \mathrm{M}$ to $40 \mu \mathrm{M}$ and the corresponding mole fraction was $0.025 \%$ to $0.8 \%$. All the solutions were freshly prepared to minimize the hydrolysis of the NHS esters. The silanized microfluidic channels were then labeled by continuously flowing 150 $\mu \mathrm{L}$ of NHS ester solution through the channel for 60 minutes at room teperature in a dark room. After labeling, the channels were rinsed with HEPES buffer and water before drying with nitrogen gas.

Functionalized glass slides were also labeled with the same concentrations of AF555-NHS-ester and sulfo-NHS-biotin. Slides were sandwiched betwen two glass coverslips with $30 \mu \mathrm{L}$ of solution covering each side and incubated at room temperature for 60 minutes. The slides were then rinsed with water and dried with nitrogen gas.

Based on the detection over a wide range of labeling concentrations, the standard mixture solution was set at $2.5 \mu \mathrm{M}$ AF555-NHS-ester (the mole fraction of $0.025 \%$ ) and $5 \mathrm{mM}$ sulfo-NHS-biotin for direct quantification and real-time monitoring.

\section{Labeling with fluorogenic reagents}

Non-fluorescent NDA was dissolved in methanol at a concentration of $1 \mathrm{mM}$. For the detection of surface amine groups inside microchannels, $200 \mu \mathrm{L}$ NDA solution was mixed with $200 \mu \mathrm{L}$ sodium borate buffer $(10 \mathrm{mM}, \mathrm{pH} 8.0)$ and $50 \mu \mathrm{L}$ soldium cyanide solution $(10 \mathrm{mM})$ prior to injection. The mixture was introduced to the channel surfaces for 10 minutes to generate fluorescent products $\left(\lambda_{\text {excitation }}=350 \mathrm{~nm}\right.$, $\lambda_{\text {emission }}=410-451 \mathrm{~nm}$ ) by reaction with amine groups as shown in Figure 3.4. Fluorescence intensities were observed under the fluorescence microscope. For the detection of amine groups in solutions, $50 \mu \mathrm{L}$ of samples were added into the mixture and the aliquot was measured with a Nanodrop 3300 fluorospectormeter.

\subsubsection{Fluorescence Imaging and Intensity Measurements}

All fluorescence experiments were conducted in microfluidic channels under buffer flow unless otherwise specified. The device was mounted onto the inverted micro- 


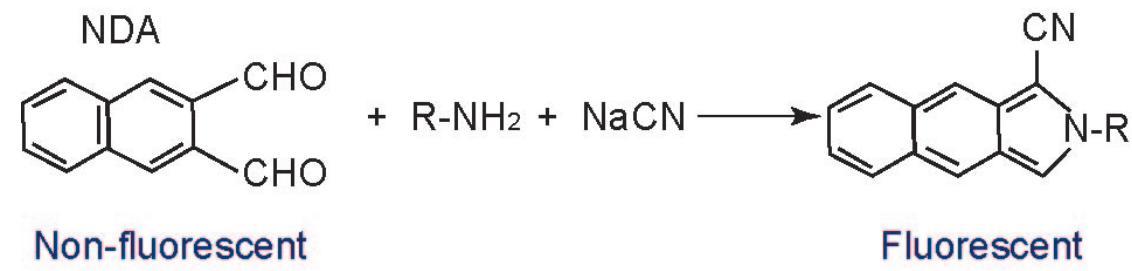

Figure 3.4: Schematic of fluorogenic reaction using NDA to label amine groups on the surfaces of the microchannels or in solution.

scope with the 10x, 0.2 NA objective and fluorescence images were captured with the CCD camera. AF555 dye was excited at $537 \pm 25 \mathrm{~nm}$ and the emission was observed at $610 \pm 96 \mathrm{~nm}$. Controlled fluid flow was achieved by pressurizing the headspace of sealed autosampler vials. Fluorescence images were acquired with an exposure time of $1 \mathrm{~s}$. Fluorescence intensity of each channel was analyzed with Image J software. Each experimental condition was performed in triplicate.

Non-specific adsorption of AF555-NHS-ester was controlled by simultaneously monitoring the fluorescence in reference channels. The intensity from the reference channel was used as background and substracted from fluorescence measurements of the other channels in the same image.

Fluorescence intensity measurements were calibrated using known AF555-NHSester concentrations in a microfluidic channel of defined depth. All molecules within the channel were assumed to contribute equally to the fluorescence signal due to the large depth of field of the 10x microscope objective used.

\subsubsection{In situ Stability Measurements}

Microfluidic channels were silanized with APTMS as described earlier and labeled with the standard mixture of $2.5 \mu \mathrm{M}$ AF555-NHS-ester and $5 \mathrm{mM}$ sulfo-NHS-biotin. The samples were then exposed to various conditions including $0.1 \mathrm{M} \mathrm{NaOH}$ solution, water, and PBS buffer ( $\mathrm{pH}$ 7.4). Each solution was tested in a separate channel. Channels were exposed for six 10-minute intervals with each solution, and between each interval the solutions were changed to HEPES buffer for fluorescence imaging. All images were acquired at $1 \mathrm{~s}$ exposure time on the CCD. Each condition was tested for a total of 60 minutes. All experiments were done in triplicate, and the relative fluorescent intensities were normalized by the starting intensity. 


\subsubsection{Fluorescence Imaging of Immobilized Proteins}

In transparent microchannel systems, immobilized insulin fibrils, $\alpha \mathrm{S}$ fibrils and rAcGFP1 on the microchannel surfaces were detected by fluorescence microscopy.

- Immobilized insulin seed fibrils were exposed to ThT solutions $(100 \mu \mathrm{M}$ in $50 \mathrm{mM}$ glycine buffer at $\mathrm{pH}$ 8.0) for 10 minutes, rinsed with the glycine buffer and imaged with exposure time of $1 \mathrm{~s}$.

- Immobilized $\alpha \mathrm{S}$ fibrils were exposed to ThT solutions $(100 \mu \mathrm{M}$ in $50 \mathrm{mM}$ glycine buffer at $\mathrm{pH}$ 8.0) for 10 minutes, rinsed with the glycine buffer and imaged with exposure time of $1 \mathrm{~s}$.

- The rAcGFP1 proteins $\left(\lambda_{\text {excitation, } \max }=475 \mathrm{~nm}, \lambda_{\text {emission, } \max }=505 \mathrm{~nm}\right)$ that were covalently immobilized on the microchannel surfaces, were imaged with $1 \mathrm{~s}$ exposure time after the protein solutions were rinsed.

For the imaging of all the three proteins, the Zeiss filter set 44 $\left(\lambda_{\text {excitation }}=475 \pm 40 \mathrm{~nm}, \lambda_{\text {emission }}=530 \pm 50 \mathrm{~nm}\right)$ was used.

\subsubsection{SMR Kinetic Measurements}

Torsional SMR chips, as presented in Section 1.2, were used to measure the elongation kinetics of $\alpha \mathrm{S}$ and insulin fibrils. The setup comprises an SMR chip with a $3 \times 8 \mu \mathrm{m}$ microchannel resonator (Section 2.1.1), an oscillator circuit with optical lever detection system (Section 1.3), and a pressure-driven fluid delivery system, as shown in Figure 3.5.

\section{Setup of the SMR chip}

The SMR chip was assembled on a metal manifold and the in- and outlets of the chip were aligned with the FEP tubes using a stereo microscope. The sealing of the assembly was then tested. Initially, the natural frequency of the SMR device (in the range of $1.2-1.5 \mathrm{MHz}$ ) was determined under gas flow. After the oscillator circuit and the optical lever detection system (Figure 3.5) were adjusted, the microchannels were filled with water. The system was then ready for further experiments. 


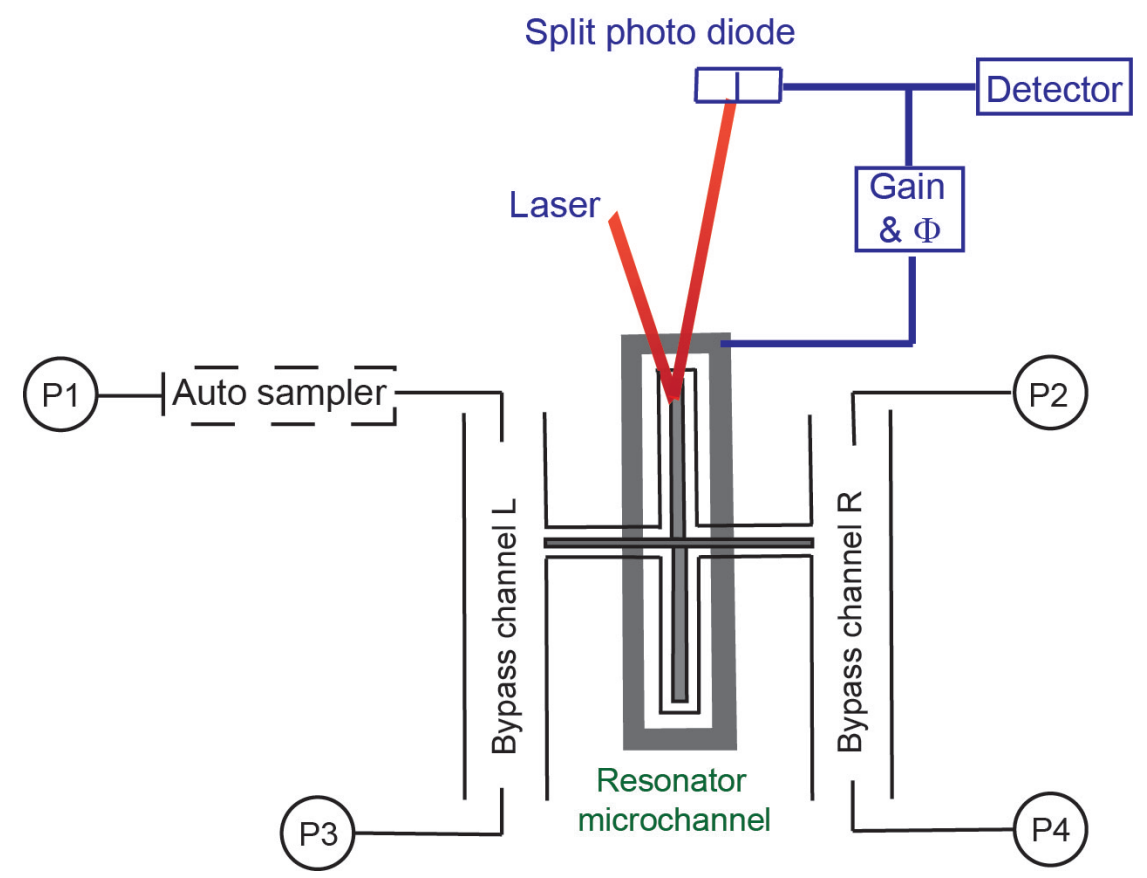

Figure 3.5: Experimental setup for the surface-based SMR measurements with an optical lever detection system and a pressure-controlled fluid delivery system.

\section{Fluid delivery}

Flanged FEP tubes were used to connect the in- and outlets of SMR chips with the running solutions, which were driven into the two large bypass channels under pressure P1-P4, as shown in Figure 3.5. The fluid flow inside the resonator microchannel was then directed by changing the pressure P1-P4. The seed fibrils were immobilized on the channel surface as described in Figure 3.3 of Section 3.3.6.1. Glycine buffer was running continuously in the bypass channel $\mathrm{R}$, while monomer solutions were injected into the bypass channel L. Before the injection, the fluid inside the microchannel was directed from channel $\mathrm{R}$ to channel L by setting P2 higher than P1. In the measurements, 10-30 $\mu \mathrm{L}$ monomer solutions were first injected into the bypass channel $\mathrm{L}$, the flow was then directed from channel $\mathrm{L}$ to channel $\mathrm{R}$ by applying P3 $<$ P1 but $(\mathrm{P} 1+\mathrm{P} 3)>\mathrm{P} 2$. Due to the small pressure drop along the bypass channel $\mathrm{L}$, the $10-30 \mu \mathrm{L}$ sample volume allowed kinetics measurements for hours with pulse-free flow through the resonator channel at rates on the scale of ca. $100 \mathrm{pL} \mathrm{s}^{-1}$. The monomer injection was conducted either directly from the pressurized vials or using an autosampler. In addition, the temperature of the SMR devices was regulated with a Peltier element. Depending on the composition of the monomer solutions, the following elongation kinetic measurements were carried out:

- Concentration-dependent elongation of $\alpha \mathrm{S}$ fibrils with monomer concentrations of $0.03,1$, and $0.3 \mathrm{mg} / \mathrm{mL}$; 
- Concentration-dependent elongation of insulin fibrils with monomer concentration ranging from 0.06 to $6 \mathrm{mg} / \mathrm{mL}$;

- Temperature-dependent elongation of insulin fibrils at temperatures between 20 and $42{ }^{\circ} \mathrm{C}$.

- Shear-effect on insulin fibril elongation at various flow rates. To evaluate the fluid velocities through the $144 \mu \mathrm{m}$ long resonator channel, microbeads were added into the running solutions to measure the transit time. A suspension of $1.5 \mu \mathrm{m}$ NIST Microbeads was prepared at a concentration of $0.1 \%$ in water and further diluted by 750 times. The suspension was ultrasonicated for 10 minutes to eliminate large aggregate particles and then mixed with $0.4 \mathrm{mg} / \mathrm{mL}$ monomer solutions. The transit time of the beads through the resonator microchannels was detected and analyzed using correlation analysis [177].

- Additive influence on insulin fibril elongation. Additives such as metal ions ( $\mathrm{NaCl}, \mathrm{ZnCl}_{2}, \mathrm{CaCl}_{2}$ and $\mathrm{MgCl}_{2}$ ), aromatic compounds (PcTS and Methylene blue) and natural products (ectoine and trehalose) were dissolved in the insulin monomer solutions $(0.4 \mathrm{mg} / \mathrm{mL})$ at different concentrations, and the kinetics were measured and compared with the kinetics obtained using monomer solutions without additives.

Furthermore, dissociation measurements were conducted in the same manner by injection of glycine buffer solutions under various conditions.

\subsubsection{Fluorospectrometer Measurements}

\section{Fluorescence measurements for AF555-NHS-ester}

The NanoDrop 3300 fluorospectrometer was used to measure the concentration of fluorescent AF555 molecules cleaved from borofloat glass slides. To remove bound fluorophores from the surface, the slides were incubated at room temperature in $60 \mu \mathrm{L}$ of $0.1 \mathrm{M} \mathrm{NaOH}$. In addition, the fluorophore concentrations were calibrated with known AF555-NHS-ester concentrations in $0.1 \mathrm{M} \mathrm{NaOH}$ solution. Fluorescence emission was measured using the white light-emitting diode (LED) excitation source (500-680 nm) of the NanoDrop with emission monitored at $565 \mathrm{~nm}$. 


\section{Fluorescence measurements for amyloid fibrils using ThT assay}

ThT was dissolved in glycine buffer $(50 \mathrm{mM}, 100 \mathrm{mM} \mathrm{NaCl}, \mathrm{pH}$ 8.0) with a stock concentration of $100 \mu \mathrm{M}$. In the fluorescence measurements, glycine buffer was used as the blank sample and $3 \mu \mathrm{L}$ of fibril samples were mixed with $27 \mu \mathrm{L}$ of ThT solution. Since ThT dye undergoes a characteristic $120 \mathrm{~nm}$ red shift of its excitation spectrum after binding specifically to amyloid fibrils [157], the fluorescence emission of the mixture solutions was measured using the blue light-emitting diode (LED) excitation source $(470 \pm 10 \mathrm{~nm})$ of the NanoDrop with emission monitored at $506 \mathrm{~nm}$. The position of the emission cursor was determined using the fluorescence profiler module of the NanoDrop 3300 programm.

\subsubsection{UV-VIS Spectrometer Measurements \\ Determination of AF555-NHS-ester concentrations}

Blank measurements were performed using buffer solutions. Then $3 \mu \mathrm{L}$ of AF555NHS-ester solutions were pipetted directly onto the pedestal and the absorption spectra were recorded using Microarray module of the NanoDrop 2000c software. By selecting AF555 dye, the spectrum cursors were placed at the wavelength of $650 \mathrm{~nm}$ and the concentrations were calculated automatically.

\section{Determination of protein concentration}

The blank measurements were done with buffer solutions and the pedestal surfaces were then cleaned. Then $3 \mu \mathrm{L}$ of protein (insulin or GFP) solutions were pipetted directly onto the pedestal and the absorption spectra were recorded with the Protein A280 module of the NanoDrop 2000c programm. The concentrations were calculated automatically using the general form of the Beer-Lambert equation.

\subsubsection{X-ray Photoelectron Spectroscopy Analysis (XPS)}

Surface characterization by XPS was performed by Sven Hartwig at the Fraunhofer Institute for Surface Engineering and Thin Films IST (Braunschweig). Open silicon substrates were analyzed using a PHI 5500 Multi-Technique X-ray photoelectron spectrometer system from Perkin-Elmer equipped with a Mg K X-ray source with energy of $1253.6 \mathrm{eV}$. The elemental composition of samples was obtained from 
survey spectra, and collected at pass energy of $187.85 \mathrm{eV}$.

\subsubsection{Electron Microscopy}

Electron microscopy (EM) was used for morphological characterization of insulin fibrils in the Facility of Electron Microscopy of the Max Planck Institute for Biophysical Chemistry.

The insulin fibril solutions produced with initial $1 \mathrm{mg} / \mathrm{mL}$ monomer solutions were firstly diluted by 50-100 times with either HEPES or glycine buffer to achieve optimal resolution. The samples were then deposited on Formvar-coated 200 mesh copper grids. The samples were washed with water and stained with freshly prepared $0.75 \%(\mathrm{w} / \mathrm{v})$ uranyl acetate and examined on a Phillip CIME 12 transmission electron microscope, operated at $80 \mathrm{kV}$. The images were recorded with a slow scan CCD camera.

\subsubsection{Atomic Force Microscopy}

Atomic Force Microscopy (AFM) detection was conducted to evaluate the density of immobilized insulin seed fibrils on silicon substrates. The measurements were conducted in the laboratory of Dr. Iwan Schaap (III. Physical Institute at University of Göttingen).

Short insulin seed fibrils prepared as described in Section 3.3.3 were immobilized on $3 \mathrm{~mm} \times 6 \mathrm{~mm}$ silicon substrates at $25^{\circ} \mathrm{C}$ following the protocols described in Section 3.3.5 and Section 3.3.6.1. The samples were then imaged with a Cervantes FullMode AFM System (Nanotec, Spain) using silicon cantilevers (Olympus OMCL-AC240TS, tip radius $7 \mathrm{~nm}$, Spring constant $1.8 \mathrm{~N} / \mathrm{m}$, resonant frequency ca. $70 \mathrm{KHz}$ ). Force curves were performed on the substrate to estimate the sensitivity $(68 \mathrm{~nm} / \mathrm{V})$. The images were acquired at room temperature using amplitude modulation by oscillating the cantilever at $65 \mathrm{kHz}$ with an amplitude of $3.5-7 \mathrm{~nm}$. 


\section{Chapter 4}

\section{SMR Surface Modification and Characterization by Fluorescence Microscopy}

\subsection{Introduction}

Robust protein immobilization on the microchannel surface is required for the accurate kinetic measurements of amyloid growth by the surface-based SMR approach. In this study, diverse immobilization techniques were evaluated: $\alpha \mathrm{S}$ fibrils were immobilized via electrostatic interaction using PLL; GFP and insulin fibrils were immobilized via multi-step covalent coupling techniques.

In some immobilization techniques, many sequential steps are involved, and it is difficult to validate the success of each of these steps directly inside the SMR devices due to several challenges: (1) the inner walls are inaccessible and not optically transparent; (2) the SMR measurements cannot resolve the deposition of small functional groups; (3) re-assembly of SMR devices to the detection set-up and the re-adjustment are required after certain steps such as vapor-phase deposition of functional groups; and (4) clogging cannot be easily recognized in the SMR detection system. Therefore, transparent microchannels were designed with similar dimensions to the SMR microchannels as shown in Figure 4.1. The microchannels were imaged using fluorescence microscopy to develop robust functionalization and immobilization methods. Although the original goal was to develop the protocols for SMR devices, the methods developed in this thesis also have their own significance for other widely applied transparent microchannel systems.

PLL was initially used to immobilize $\alpha \mathrm{S}$ fibrils due to the ease of use. In the 


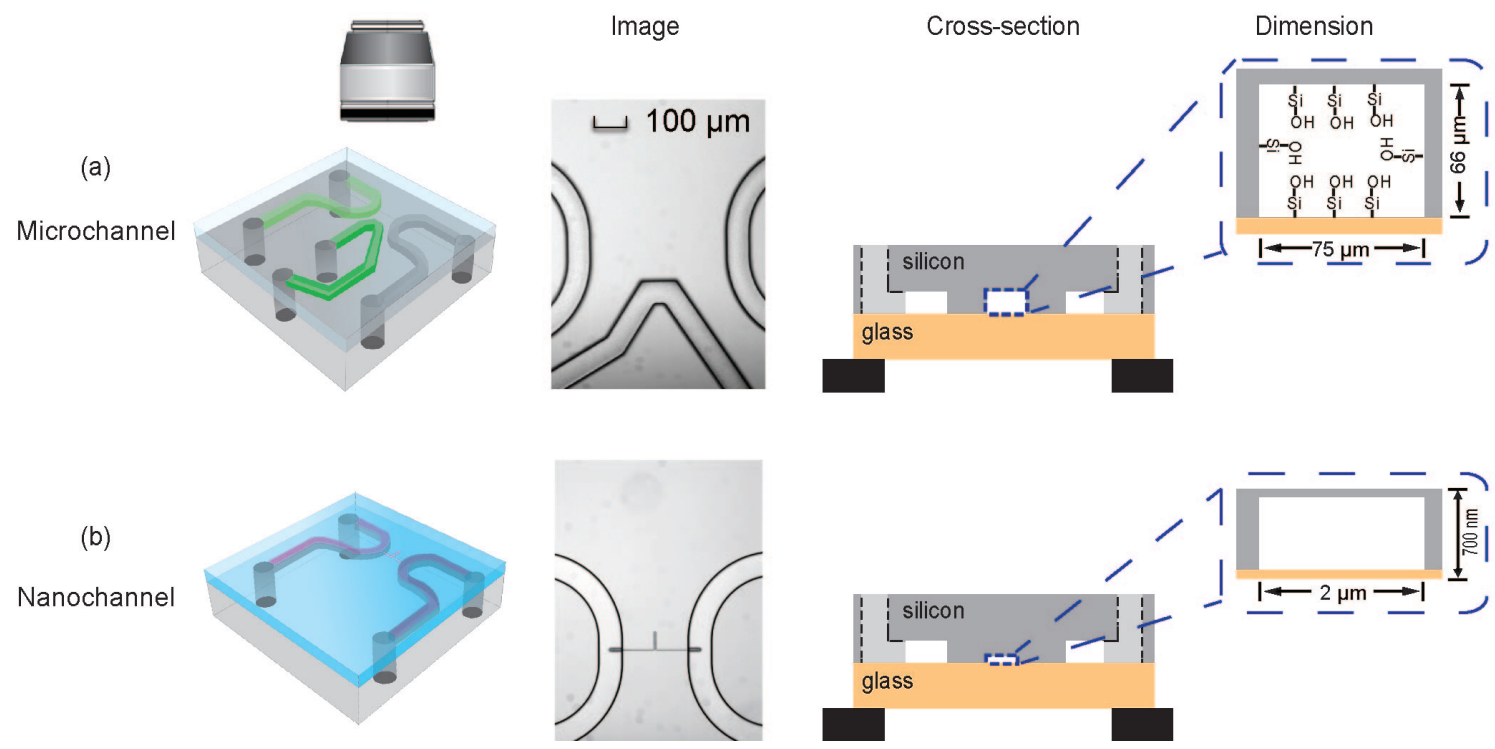

Figure 4.1: Micro- (a) and nanochannels (b) for fluorescence microscopy.

transparent microchannels, seed fibrils binds efficiently to the surfaces. However, the fluorescence detection in transparent microchannels did not allow further evaluation of non-specific binding and stability. Alternatively, covalent immobilization methods were also developed to achieve long-term stability. By comparing the quantities of differently immobilized proteins, reliable binding assays were selected and adapted to the measurements with SMR devices.

As presented in Section 2.2.1, multiple steps are often involved in the covalent immobilization of proteins on solid supports. In this study, tri- and monoalkoxysilanes were used to introduce reactive amine groups to the hydroxyl-containing surfaces, followed by further functionalization and immobilization of targeted proteins. The robustness of the initial surface functionalization step, amino-silane deposition, is very important for the success of the immobilization process. However, applying established deposition methods to the microfluidic channels is not trivial, partially due to the fact that the system is sensitive to the problems of clogging and contamination by dispersion. Moreover, there is a lack of techniques to characterize the functionalization quantitatively in closed microchannels because of several issues. First, the channel surfaces are inaccessible and the routine analysis techniques for open-surface systems, such as AFM, SEM, XPS, ellipsometry, contact angle measurement and labeling detection methods, cannot be readily applied $[28,46,58,79,173,180,199,248]$. Second, biosensor-based approaches (e.g. SPR, OWS, TLM and QCM) often require devices which have been specifically designed for the measurements, although they can quantitatively monitor the surface reactions in real-time $[55,145,147,163,175]$. Finally, the methods based on labeling techniques 
followed by cleavage, elution and detection steps are not practically applicable, since all the steps need to be compatible with the microfluidic systems and the collected eluent concentrations are much lower than the detection limit [194,248]. To address these issues, two goals in this thesis were set to the development of robust deposition methods and in-channel quantification methods.

Here a vapor-based deposition method is presented to allow robust in-channel surface functionalization with amine groups for batch production. Furthermore, the quality of functionalization, such as homogeneity, silane layer stability and reproducibility, is assessed in situ using a novel fluorescence-based quantification method. This novel imaging-based method measures directly the surface coverage of functional groups inside assembled microchannels over a wide dynamic range. Compared to the fluorescence detection that only qualitatively shows the presence of the functional groups, the novel approach is readily applied to optimize the protocols, to maximize the surface density of functional groups and to eliminate errors due to chip-to-chip surface variability. In addition, the measured surface density of functional groups represents directly the reactive groups that are accessible and available for further coupling.

Furthermore, subsequent functionalization using glutaraldehyde and carboxymethyl-dextran were conducted to immobilize proteins. Green Fluorescent Protein (GFP) was used to asses the binding capacities using different immobilization methods. It was concluded that protein immobilization with glutaraldehyde is a more promising method, which has been further applied to study kinetics of insulin fibril growth in SMR detection systems in the following chapter.

\subsection{Electrostatic Immobilization of $\alpha \mathrm{S}$ Using PLL}

Coating glass/silicon surfaces with PLL has been widely used as the most economic method to immobilize antibodies and DNA in microarray applications [48]. In addition, the ease of use of PLL solutions allows surfaces to be functionalized within one step with positive charge that can interact strongly with negatively charged molecules. In the transparent microchannels, PLL was used to electrostatically immobilize $\alpha$ S fibrils (pI 4.7) at $\mathrm{pH} 7$.

The fibrils in PBS buffer at $\mathrm{pH} 7$ were initially obtained as more than $2 \mu \mathrm{m}$ long fibrils (Figure 4.2 (b)) and ultrasonicated into short seed fibril of $146 \pm 74 \mathrm{~nm}$, 
as depicted in Figure 4.2 (c). After the seed fibrils were immobilized on the PLL-coated channel surfaces (Channel "L" of Figure 4.3 (a)), ThT molecules were injected into the microchannel that allowed imaging by fluorescence microscopy. ThT, with structure shown in Figure 4.4 (a), is a fluorogenic label for $\beta$-sheet-rich fibrils. When ThT was bound to the amyloid fibrils, the fluorescence intensity was enhanced dramatically with excitation and emission maxima at approximately 440 and $490 \mathrm{~nm}$ (Figure 4.4 (b)). The significantly higher intensity of channel "L" compared to the reference channel "R" in image (a) indicates that PLL can efficiently immobilize $\alpha$ S-fibrils to the inner walls of the microchannels.
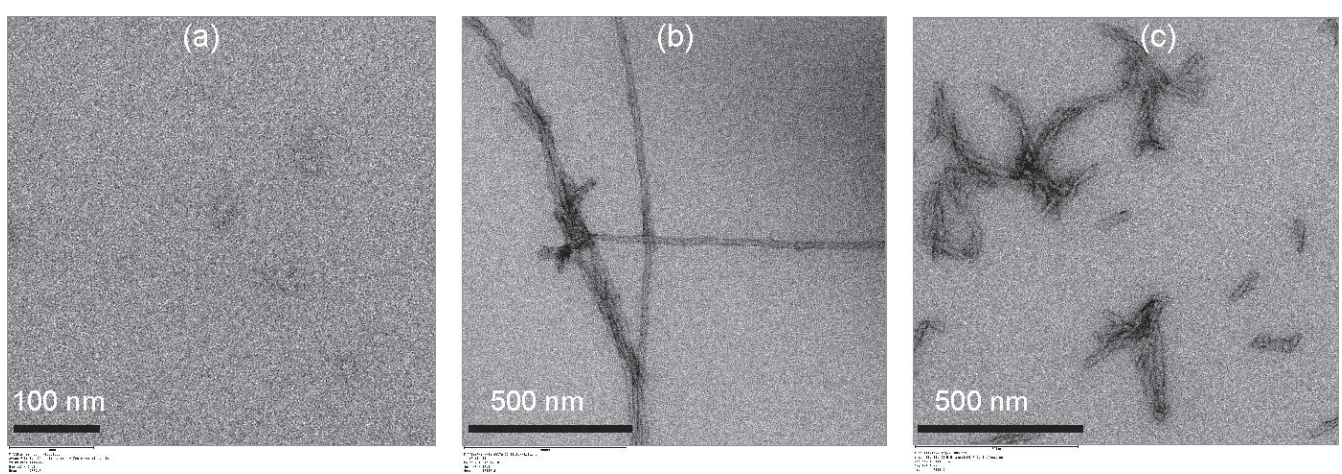

Figure 4.2: Electron micrographs of (a) $\alpha \mathrm{S}$ monomers, (b) fibrils and (c) sonicated fibrils.

However, the stability of the fibrils on the surfaces could not be further assessed, as any signal decrease could also be the result of the unknown photobleaching rate of the ThT-labeled fibrils. Furthermore, quantitative evaluation of binding efficiency (surface density of the immobilized fibrils) using PLL-modification was not possible by this ThT-based fluorescence detection. It was highly possible that the labeled ThT molecules could alter the fibril properties and thus further interfere with the aggregation process. Evidence for this is shown in Figure 4.3 (b). In the channel "L" of image (b-I), $\alpha \mathrm{S}$ fibrils in solution were introduced to the native surface of the microchannel and the subsequent ThT flow did not result in an intensity increase. This is in agreement with the fact that negatively charged $\alpha \mathrm{S}$ fibrils do not bind to the negatively charged $\mathrm{SiO}_{2}$-surface. However, when the $\alpha \mathrm{S}$ fibrils were firstly mixed with ThT in solution and the mixture was injected into the channel "L" of image (b-II), significant fluorescence signal was observed, indicating that the fibrils stained with ThT remained on the native channel surface. This observation evidently confirmed that the binding of ThT-molecules could promote stronger interaction between $\alpha \mathrm{S}$ and the native glass/silicon surface.

However, the binding mechanism of ThT to amyloid fibrils is poorly understood 
(a) PLL $+\alpha$ S fibril + ThT

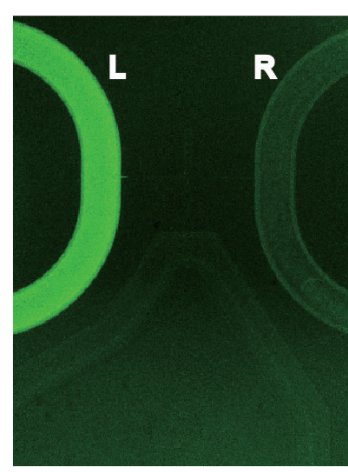

(b) (I) $\alpha \mathrm{S}$ fibril + ThT

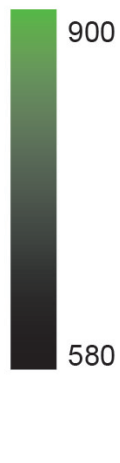

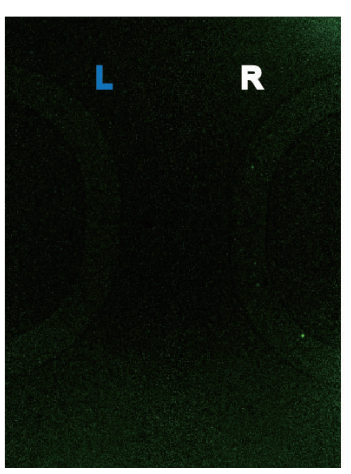

(II) $\alpha$ S fibril/ThT

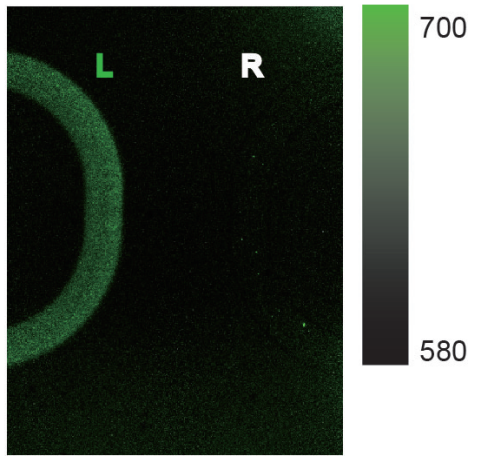

Figure 4.3: Fluorescence images showing $\alpha$ S-fibril immobilization on microchannel surfaces. All channel "R" are reference channels without surface functionalization, shortly exposed to ThT solutions and then filled with buffer solutions. The injection sequences of the solutions in channel "L" are respectively: (a) water - PLL - water - buffer - $\alpha \mathrm{S}$ fibrils - buffer - ThT - buffer; (b) buffer - $\alpha \mathrm{S}$ fibrils - buffer - ThT - buffer (I) and buffer - $\alpha \mathrm{S}$ fibrils with ThT - buffer (II).

and the unknown binding stoichiometry of ThT to the fibrils does not allow to correlate the revealed fluorescence intensity with the surface density of the immobilized fibrils. Several studies on the mechanism of ThT-fibril interaction have recently been reported. Among several hypotheses, the "channel" model of ThT-fibril interaction suggests that ThT molecules are aligned parallel to the long axis of the fibril and are likely located at the side-chain grooves of the $\beta$-sheet structure $[20,80,140]$. However, the hypothesis can not provide any further information to quantify the ThT-fibril binding stoichiometry.

PLL was further applied to bind proteins in the label-free SMR detection systems (Section 5.1.2), and the stability using this immobilization technique for kinetic measurements will be discussed in Section 5.1.2. 
(a)

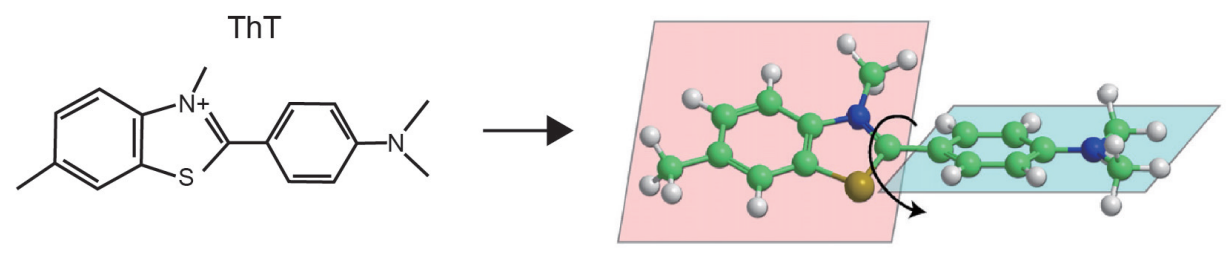

(b)

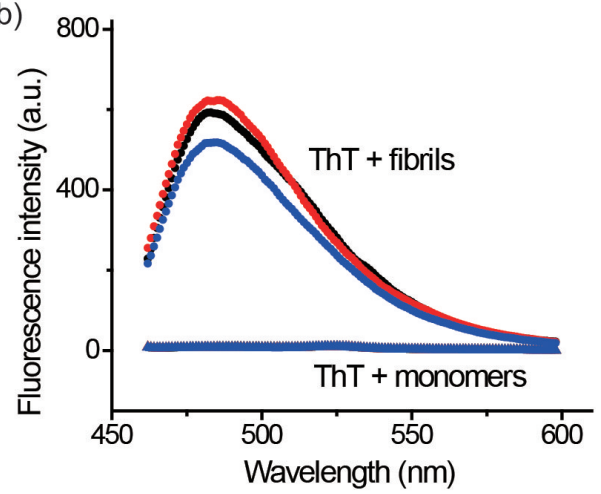

(c)

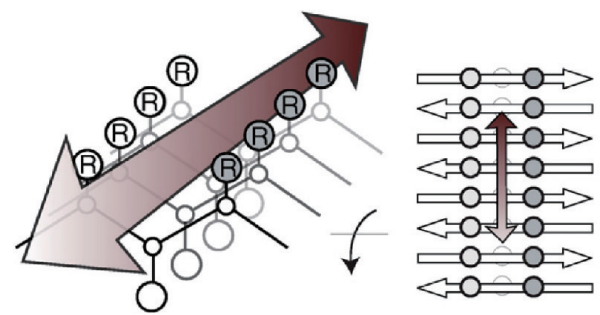

Figure 4.4: ThT binding to amyloid fibrils. (a) Schematic of ThT structure and the 3D representation adapted from Ref. [20]. (b) Plot showing fluorescence emission of ThT solution in the presence of $\alpha$ S fibrils and monomers. (c) Proposed "channel" model of ThT binding to fibril-like $\beta$-sheets in Ref. [20].

\subsection{Covalent Immobilization of Proteins}

\subsubsection{In-Channel Surface Functionalization}

\subsubsection{Vapor-Based Surface Functionalization with Aminosilanes}

Amine functional silanes are among the most common and versatile coupling agents for the functionalization of silicon and glass surfaces. Unfortunately, standard silanization protocols that are developed for open surface systems often yield poor reproducibility when translated to closed microfluidic systems. In this study, aminosilanes were initially deposited by liquid-phase deposition using toluene. Using fluorescence labeling with fluorogenic NDA molecules, the qualitative detection often showed that the microchannels were clogged by self-assembled aggregation of aminosilanes, as exemplified in Figure 4.5 (a). This problem was overcome by vapor-phase deposition, which consistently modified both the large bypass channels and the small $700 \mathrm{~nm}$ high nanochannels with a homogeneous silane layer. The process used for vapor-phase silanization is described in Section 3.3.5.

\subsubsection{Surface Quantification of Aminosilane Functionalization}

A fluorescence-based method is demonstrated here to quantify surface functional groups in closed micro- and nanofluidic channels in situ [258]. Fluorescence 
(a) Liquid phase deposition

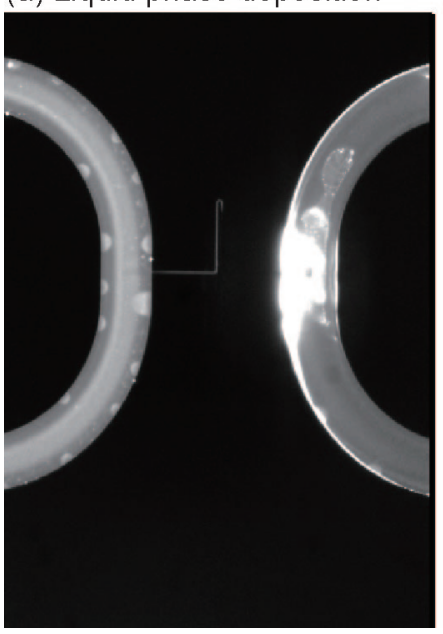

(b) Vapor phase deposition

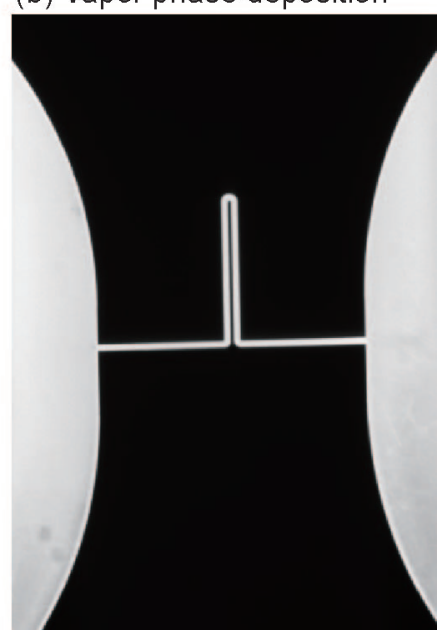

Figure 4.5: Surface modification with APTMS by liquid phase silanization (a) and vapor phase silanization (b). Image (b)was taken by applying the new quantitative fluorescence method.

microscopy is a widely applicable detection technique with the advantages of high sensitivity and ease of use $[58,106,112,122,199]$. However, quantitative interpretation of fluorescence from the channel surface is not trivial. Initially, the amine functionalized surface was labeled with a pure solution of fluorescent AF555-NHS-ester. As shown in Figure 4.6 (a \& c), the fluorescence intensity measured for labeling concentrations of 5 and $10 \mu \mathrm{M}$ showed no change, suggesting that surface saturation had been reached. Interestingly, under constant illumination it was observed that the fluorescence intensity rose for the first ca. $500 \mathrm{~s}$ before exhibiting a continual decrease. This behaviour might be attributed to the opposing effect of self-quenching and photobleaching.

Fluorescence self-quenching has been reported to occur in a variety of systems, including concentrated solutions and labeled proteins [44,79,271,277]. In microfluidic systems, self-quenching of closely packed fluorescent molecules poses an important challenge for accurate quantification of surface coverage. The potential to mitigate self-quenching was explored here by controlling the distance between immobilized fluorescent molecules. To achieve this, a non-fluorescent competitor, sulfo-NHS-biotin, was introduced into the AF555-NHS-ester solution to dilute the surface concentration of fluorescent molecules. Sulfo-NHS-ester was selected here not for its functionality of biotin, but for its similar size, good solubility, and expectedly similar reaction kinetics to AF555-NHS-ester.

The effect of limiting the fluorescent labeling density was clearly observed by comparing the brightness of the three channels in Figure 4.6 (c). The left channel was labeled using a solution of $5 \mu \mathrm{M}$ AF555-NHS-ester in a background of $5 \mathrm{mM}$ 

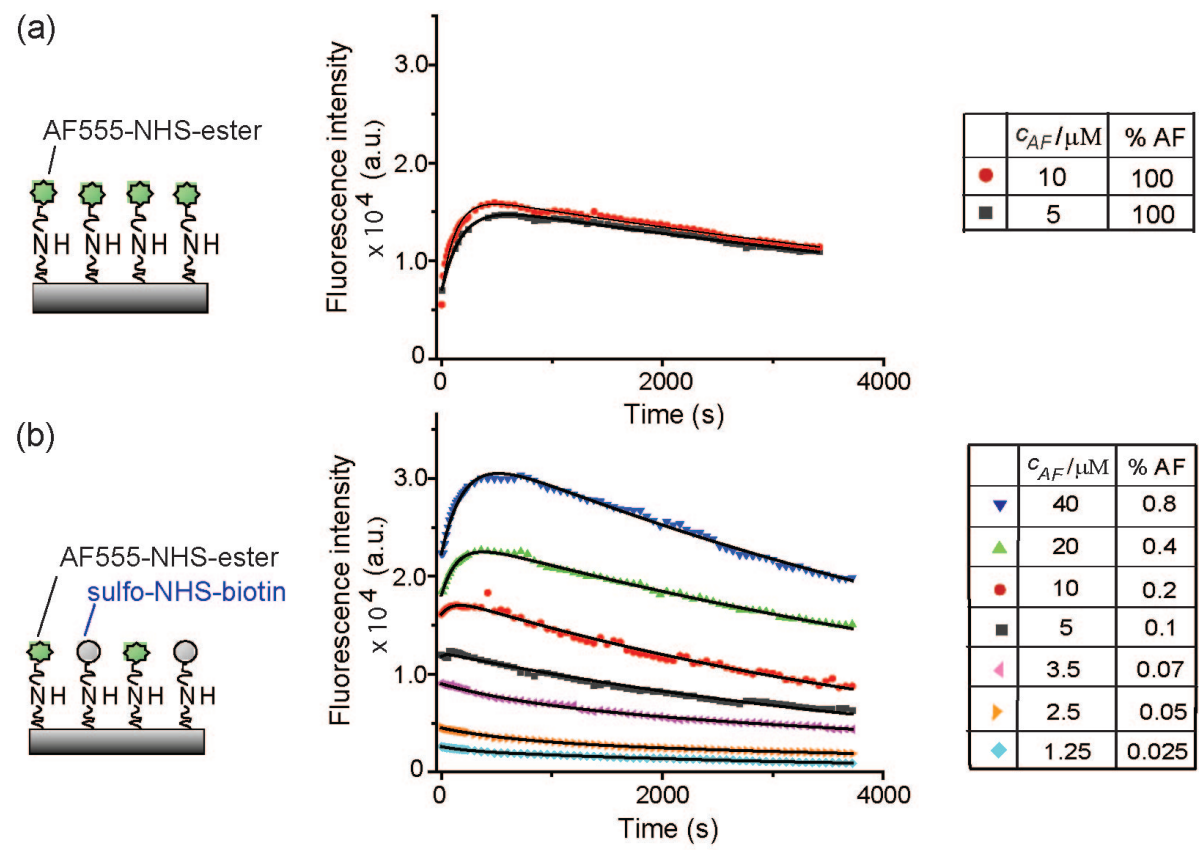

(c)
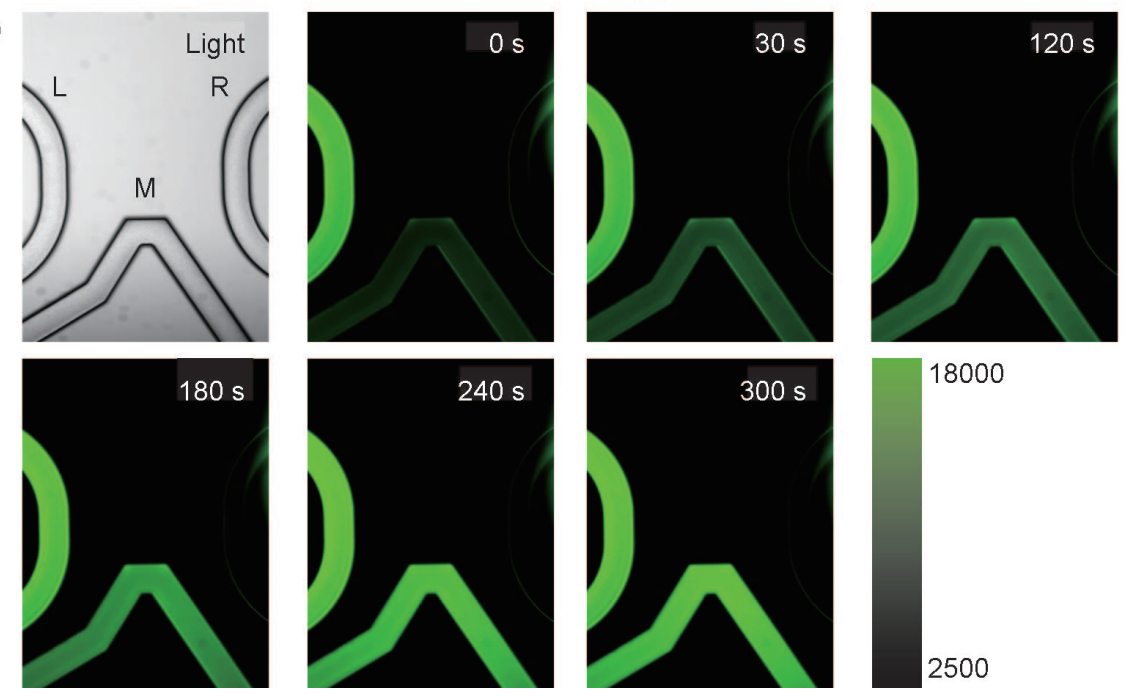

Figure 4.6: Fluorescence intensity profiles and concentration tables for microchannels immobilized with (a) AF555-NHS-ester alone and (b) mixtures of fluorescent AF555-NHSester and non-fluorescent sulfo-NHS-biotin. Solid lines represent the least squares fits according to Equation 4.1. (c) Bright field and fluorescence images at time 0-300 s of three microchannels silanized and labeld with AF555-NHS-ester $(5 \mu \mathrm{M})$ and sulfo-NHS-biotin $(5 \mathrm{mM})(\mathrm{L})$; silanized and labeled with AF555-NHS-ester $(5 \mu \mathrm{M})$ alone $(\mathrm{M})$; unsilanized and labeled with AF555-NHS-ester $(5 \mu \mathrm{M})$ alone (R).

sulfo-NHS-biotin, while both the middle channel and the unsilanized right reference channel were exposed to $5 \mu \mathrm{M}$ AF555-NHS-ester alone. Fluorescence in the unsilanized reference channel remained unchanged after incubation of AF555-NHS-ester alone, indicating that non-specific adsorption was below the detection limit. At the initial point of exposure, higher intensities were observed in the sparsely labeled left channel compared to the middle channel. This relationship then reversed after 
prolonged illumination, indicating that under heavy labeling conditions quenching was present.

Time course measurements of fluorescent intensity for various AF555-NHS-ester and sulfo-NHS-biotin concentration ratios are shown in Figure 4.6 (b). The total concentration of both species was maintained at $5 \mathrm{mM}$ to minimize potential differences in labeling efficiency. The fluorescence intensity decreased monotonically with illumination time at low concentration ratios of AF555-NHS-ester (0.025\%$0.1 \%$ ). However, for higher ratios $(\geq 0.2 \%)$, a similar trajectory to Figure 4.6 (a) was observed and this non-monotonic behaviour became more evident as the concentration of AF555-NHS-ester increased.

The observed non-monotonic trajectory of the fluorescence signals can be well fitted with Equation 4.1 (established by Dr. Y.X. Mejia in the research group), which stems from the interplay between photobleaching and self-quenching phenomena. The fitted lines are shown in Figure 4.6(a \& b).

$$
I=\left(A_{1} e^{-t / \tau_{p h 1}}+A_{2} e^{-t / \tau_{p h 2}}\right)\left(1-A_{3} e^{-t / \tau_{\text {dequench }}}\right)
$$

Here, the photobleaching, described in the first term with two decay half-life $\tau_{p h 1}$ and $\tau_{p h 2}$ and parameters of $A_{1}$ and $A_{2}$, is suggested to follow multi-exponential behavior in different environment, especially in the case of bound molecules $[18,144,237,247]$. In the second term, the fraction $\left(A_{3}\right)$ of quenched fluorophores that dequench with time constant $\tau_{\text {dequench }}$ describes the intensity increase. The fitting results are listed in Table 6.1 with fitting errors in Table 6.2 in the Appendix. It was concluded that fluorophors were not quenched $\left(A_{3}=0\right)$ at concentrations lower than $3.5 \mu \mathrm{M}$. For higher concentrations, $A_{3} \neq 0$, quenching was present. The photobleaching could be described as a single exponential decay with negligible $A_{2}$-values because the inclusion of $A_{2}$ in fits provided no improvement in the fitting quality.

The mechanisms behind self-quenching are in general complex and highly system-dependent [44]. Therefore, the full theoretical interpretation of the quenching and photobleaching dynamics of surface bound fluorophores remains an interesting problem and needs to be further studied not only in this system but also for other labeling-based systems.

Fluorescence intensity as a function of fluorophore concentration was calibrated using solutions of known AF555-NHS-ester concentrations inside the microchannels as shown in the inset of Figure 4.7(a). Using this calibration, the measured den-

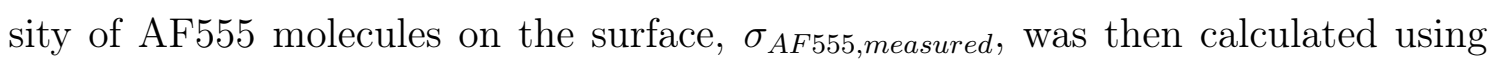


(a)

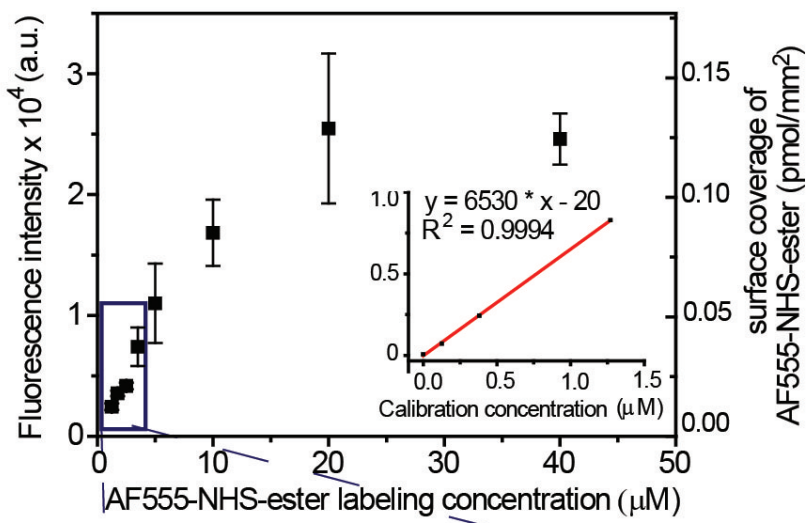

(b)

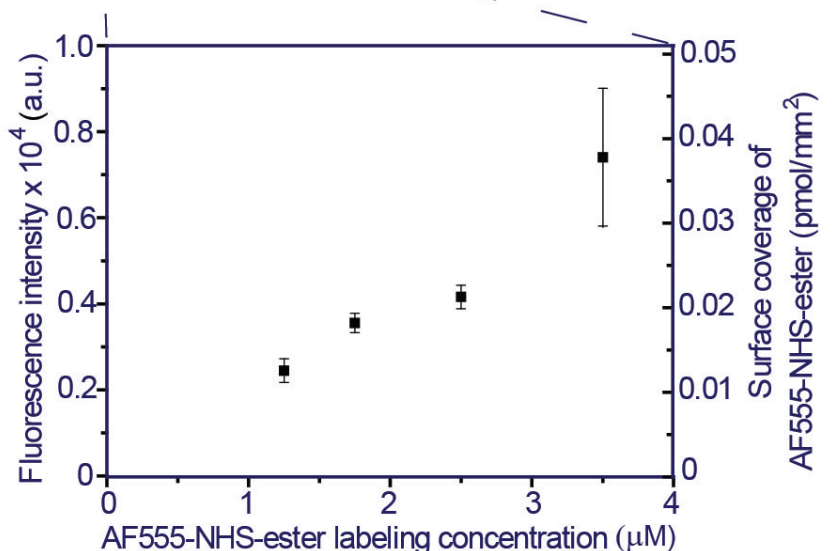

(c)

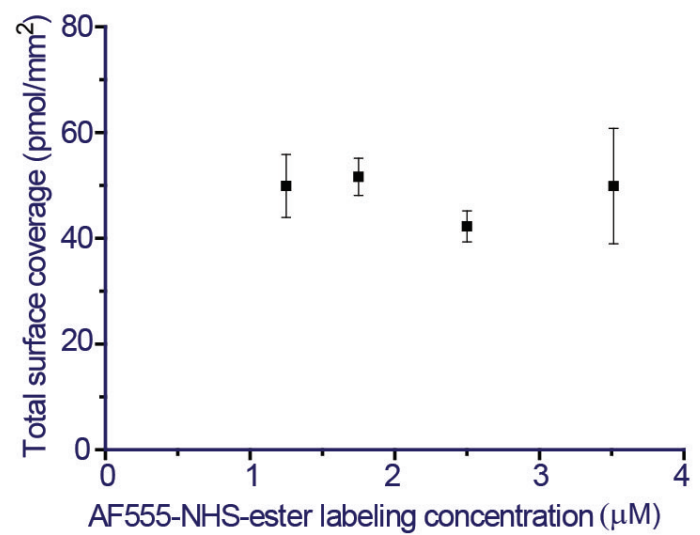

Figure 4.7: Fluorescence intensity and the corresponding surface coverage of different AF555-NHS-ester concentrations resulting from the competitive surface reaction. (a) Including higher fluorophore concentrations. (b) Only with fluorophore concentrations lower than $3.5 \mu \mathrm{M}$. (Inset) Calibration plot of fluorescence intensity versus concentration. (c) Calculated total surface coverage of the microchannels using Equation 4.2. Error bars indicate \pm 1 standard deviation calculated from triplicate experiments.

Equation 4.2:

$$
\sigma_{\text {total }, \text { measured }}=\frac{\sigma_{A F 555, \text { measured }} *\left(c_{A F 555}+c_{\text {biotin }}\right)}{c_{A F 555}}
$$

where $c_{A F 555}$ and $c_{\text {biotin }}$ are the labeling concentrations of AF555-NHS-ester and 
sulfo-NHS-biotin, respectively. This equation is based on the assumption that both species undergo the same chemical reaction kinetics and the coupling processes are reaction limited. First, both labeling species have the same NHS-ester coupling chemistry. The rate constant of the aminolysis reaction determined experimentally under similar $\mathrm{pH}$ and temperature conditions is reported to be $578 \pm 13 \mathrm{M}^{-1} \mathrm{~s}^{-1}$ $[17,51,250]$. As the rate determining step in this reaction is the nucleophilic attack of the non-protonated amino-groups, it is expected that this rate should be very similar for both labeling species. Second, the reaction rate of a diffusion-limited reaction is estimated to be on the order of $10^{9}-10^{10} \mathrm{M}^{-1} \mathrm{~s}^{-1}$, which is orders of magnitude faster than the NHS-ester to amine reaction rate [17]. In the microchannel systems of this study, the binding rate of the competing labeling species is thus not diffusionlimited but reaction-limited, eliminating any possible effect the relative size of the ligands could have.

As such, the amount of each bound species was well approximated by the relative concentrations of ligands in solution. However, in case that different coupling chemistries are applied for the two species, the stoichiometry on the surface will not only depend on the relative concentrations in solution, but also on the ratio of the binding rate constants.

$$
\frac{\sigma_{\text {dye }}}{\sigma_{\text {competitor }}}=\frac{k_{\text {dye }}}{k_{\text {competitor }}} * \frac{c_{\text {dye }}}{c_{\text {competitor }}}
$$

Here, $\sigma_{d y e}$ and $\sigma_{\text {competitor }}$ are the surface coverage of both products, $c_{\text {dye }}$ and $c_{\text {competitor }}$ are the initial concentrations in solution, and $k_{\text {dye }}$ and $k_{\text {competitor }}$ denote the binding rate constants to surface functional groups respectively. By knowing the ratio of rate constants and measuring surface coverage of fluorophore molecuels $\sigma_{d y e}$, the total surface coverage can be determined using Equation 4.4:

$$
\sigma_{\text {total }}=\sigma_{\text {dye }} *\left(1+\frac{k_{\text {competitor }}}{k_{\text {dye }}} * \frac{c_{\text {competitor }}}{c_{\text {dye }}}\right)
$$

In the absence of self-quenching at low fluorescence labeling concentrations, a linear correlation between fluorescence intensity and surface coverage of fluorophore was achieved, as shown in Figure 4.7(b). The small deviations from linearity are likely due to variations in concentration during preparation caused by hydrolysis. In contrast, the fluorescence intensity of surfaces labeled with mixture solutions that had larger fluorophore fractions deviated significantly from linearity as depicted in Figure $4.7(\mathrm{a})$. 


\subsubsection{Quantification of Vapor-Deposited APTMS and APDMMS Layers}

The optimized fluorescence labeling method was applied to quantify the surface coverages of aminosilane layers formed using APTMS and APDMMS.

By the APTMS deposition, an average total amine coverage inside the $3 \times 8 \mu \mathrm{m}$ channel was obtained to be $55 \pm 9 \mathrm{pmol} / \mathrm{mm}^{2}$ using Equation 4.2. This result was independent of the exact ratio of AF-NHS-ester and sulfo-NHS-biotin for mixtures in the range $c_{A F}=1.25-3.5 \mu M$ as shown in Figure 4.7 (c). Similar results were obtained when the experiment was conducted in nanofluidic channels with $700 \mathrm{~nm}$ height. Based on the detection over a wide range of labeling concentrations, a solution of $2.5 \mu \mathrm{M}$ AF555-NHS-ester (the mole fraction of $0.05 \%$ ) and $5 \mathrm{mM}$ sulfo-NHS-biotin was set as a standard mixture solution for direct quantification and real-time monitoring.

The measured surface coverage was consistent with the reported amine density $\left(3-150 \mathrm{pmol} / \mathrm{mm}^{2}\right)$ for an open silicon surface measured after cleavage of labeled molecules with UV/VIS detection [179,181]. In contrast, by labeling with fluorescent AF555-NHS-ester alone, initial intensities would only correspond to $0.12-0.14 \mathrm{pmol} / \mathrm{mm}^{2}$-values that were at least an order of magnitude less than the true surface coverage. This illustrates the importance of controlling the density of fluorophores for surface coverage quantification. To further corroborate the reliability of the quantification method, vapor phase APTMS silanization was also performed on open borofloat glass slides $\left(\sim 240 \mathrm{~mm}^{2}\right)$ and the surface coverage was quantified again by cleaving the labeled molecules and detecting with a fluorospectrometer. The total calculated surface coverage of $63 \pm 21 \mathrm{pmol} / \mathrm{mm}^{2}$ was consistent with our microscopy-based measurements of labeled surfaces inside the microchannels.

Assuming that the footprint of an APTMS molecule is around $1.9 \mathrm{~nm}^{2}$, a monolayer surface coverage would yield $0.87 \mathrm{pmol} / \mathrm{mm}^{2}$ [145]. Therefore, the aminosilane coating on the surface of the microchannel was probably multilayered, making some of the embedded amine groups inaccessible to surface labeling. As such, the surface coverage measured with the fluorescence labeling method represented the effective number of accessible reactive sites only. This could be quite advantageous since practical applications of aminosilane layers typically require the validation of binding capacity rather than surface coverage.

The fluorescence labeling method was further applied to quantify the surface 
coverage of aminosilane layers using APDMMS. Monoalkoxysilanes have been suggested to form a self-assembled monolayer on glass/silicon surfaces with the suggested advantage of better uniformity with shorter length of the interfacial region compared to multilayers [69]. The surface coverage was quantified with the proposed standard labeling condition identical to those used in the stability tests (a mixture of $2.5 \mu \mathrm{M}$ AF555-NHS-ester and $5 \mathrm{mM}$ sulfo-NHS-biotin). The result showed that APDMMS modified surface had $75 \%$ less accessible amine groups bound compared to the APTMS surface (Figure 4.8).

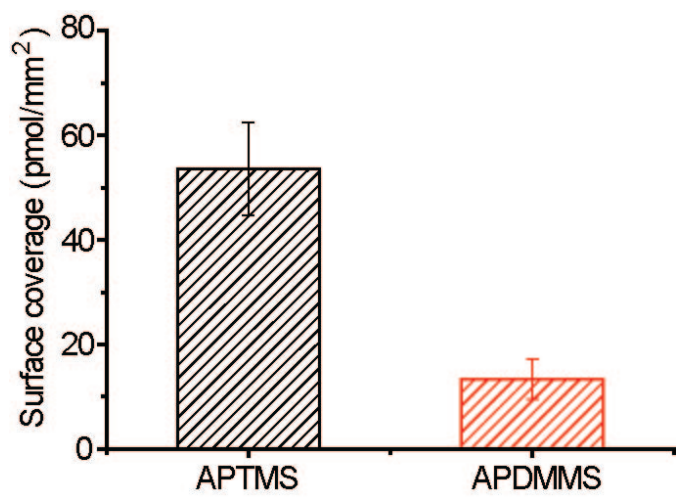

Figure 4.8: Plot showing the total surface coverage for microchannel surfaces modified with aminoalkoxysilanes APTMS and APDMMS. Error bars indicate \pm 1 standard deviation calculated from triplicate experiments.

\subsubsection{In situ Stability Monitoring of Aminosilane Functionalization}

The stability of amine functionalized microchannel surfaces under different solution conditions was monitored using real-time fluorescence imaging. The microchannels were labeled with the standard mixture of $2.5 \mu \mathrm{M}$ AF555-NHS-ester, $5 \mathrm{mM}$ sulfo-NHS-biotin and then continuously rinsed with the HEPES solution $(50 \mathrm{mM}$, $\mathrm{pH}$ 6.5). Figure 4.10(a) shows the fluorescence intensity change of labeled amine functionalized surfaces that were exposed to solutions of 1 x PBS ( $\mathrm{pH} 7.4$ ), pure water, and $0.1 \mathrm{M} \mathrm{NaOH}$ ( $\mathrm{pH} 13$ ) and then imaged by temporarily changing the solution in the channels to the HEPES solution. As AF555 is highly photostable, fluorescence loss as cause for the fluorescence signal reduction can be ruled out $[56,200]$. The fluorescence intensity remained stable for both PBS and water but decreased quickly in the presence of $0.1 \mathrm{M} \mathrm{NaOH}$ with a half-life of ca. 16 minutes.

To corroborate the results of the stability test, identically prepared open silicon surfaces were characterized with XPS analysis. Table 4.1 shows the relative chemical composition of the APTMS modified substrates after incubation in 


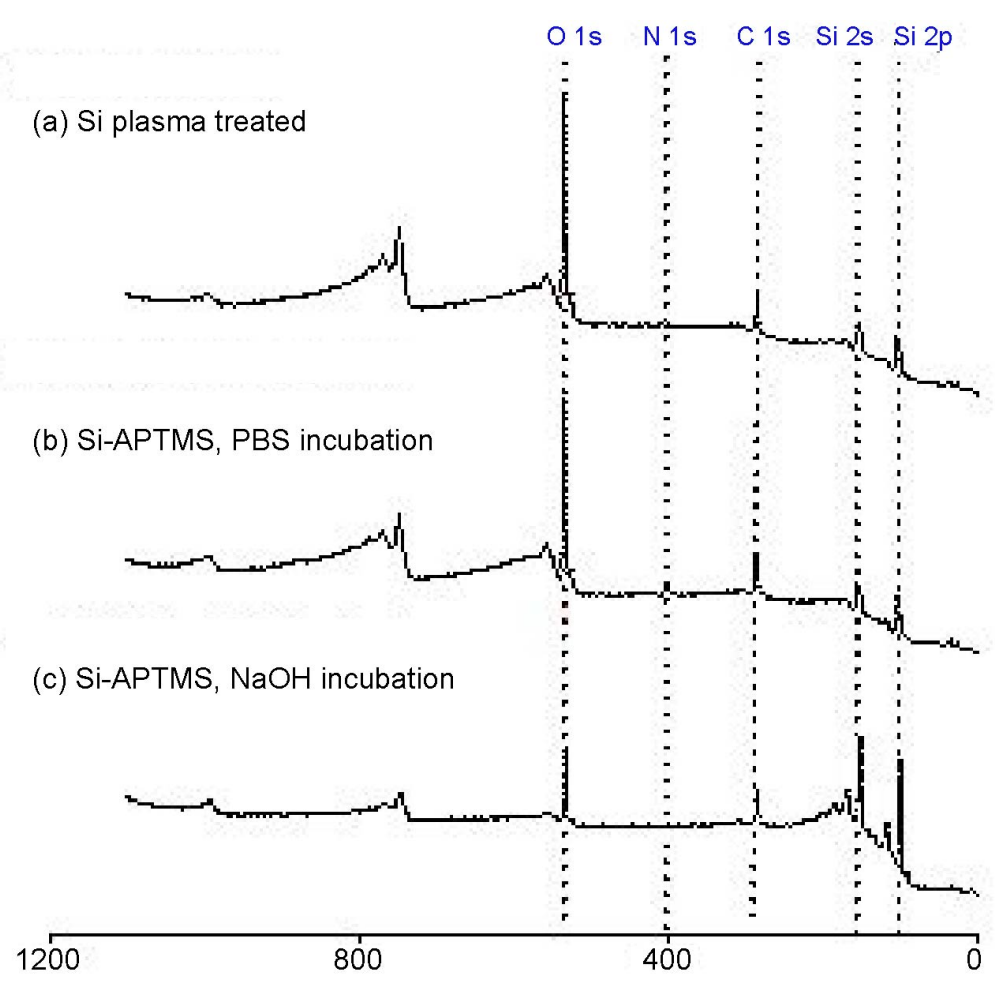

Figure 4.9: XPS survey spectra of modified silicon substrates stability in solutions.

different solutions and representative survey spectra are shown in Figure 4.9. Initially, plasma treated surfaces resulted in 50.5 at.\% oxygen from the oxide layer. At the same time, 24.5 at.\% silicon was present, along with 24.8 at.\% carbon and negligible nitrogen. Surfaces functionalized with APTMS and incubated one hour in PBS resulted in a significant increase in nitrogen to 1.5 at.\% with an uncertainty of \pm 0.2 at.\% (error estimated from background on clean silicon surfaces). In comparison, surfaces treated with $0.1 \mathrm{M} \mathrm{NaOH}$ solution for 60 minutes resulted in a substantial decrease in nitrogen content (0.1 at.\%). As elemental nitrogen was present only on the aminosilane deposited surfaces (Figure 3.2), the nitrogen composition was used as indicator for amine groups. The results verified the stability of the surface bound amine groups in PBS as well as their instability in the basic $\mathrm{NaOH}$ solution. Furthermore, these results also confirmed that the decrease in fluorescence intensity for surfaces exposed to $\mathrm{NaOH}$ in Figure 4.10 could be attributed to a degradation of the silane layer itself and not to the loss of fluorophore emission.

The stability tests further confirmed the significance of the labeling with the standard mixture for precise quantification. Figure 4.10(b) shows that the fluorescence intensity of the densely labeled surface with pure fluorophore solutions 

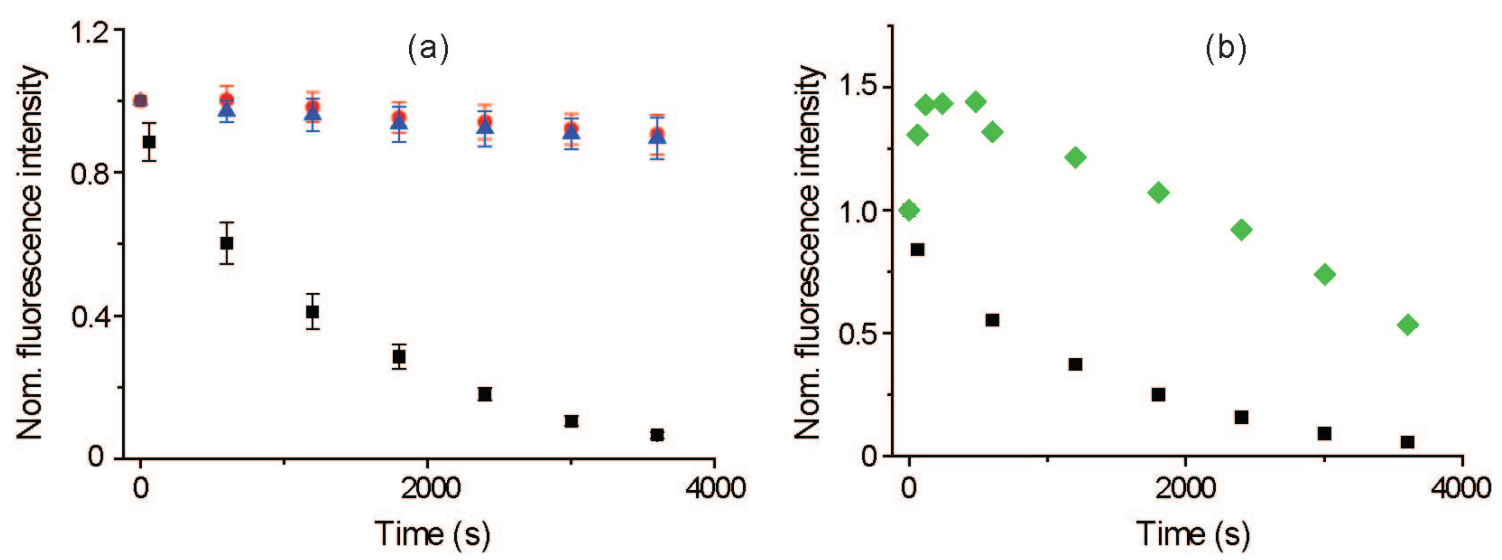

Figure 4.10: (a) Time-dependent stability measurements of APTMS modified surface rinsed with PBS buffer with pH 7.4 (red solid circle), pure water (blue solid triangle), and $0.1 \mathrm{M} \mathrm{NaOH}$ (black solid squre). Error bars indicate \pm 1 standard deviation calculated from triplicate experiments. (b)Fluorescence intensity change of APTMS modified surface labeled (black solid squre) with the standard mixture solution and (green solid diamond) with $5 \mu \mathrm{M}$ AF555-NHS-ester alone.

Table 4.1: XPS atomic concentrations of APTMS modified surfaces after incubation in different solutions. Atomic concentrations of nitrogen were used to indicate the presence of amine functional groups. The error in $\mathrm{N}($ at.\%) is estimated at 0.2 at.\% based on the background of non-functionalized, plasma treated silicon surfaces.

\begin{tabular}{ccccc}
\hline \hline Surface & $\mathrm{O}($ at.\%) & $\mathrm{N}($ at.\%) & $\mathrm{C}$ (at.\%) & $\mathrm{Si}$ (at.\%) \\
\hline Si, plasma treated & 50.5 & 0.2 & 24.8 & 24.5 \\
Si-APTMS, PBS incubation & 41.5 & 1.5 & 34.1 & 22.9 \\
Si-APTMS, NaOH incubation & 17.5 & 0.1 & 22.4 & 60.0 \\
\hline \hline
\end{tabular}

changed non-monotonically by exposure to $\mathrm{NaOH}$ solutions (green solid diamond). The initial increase in intensity could be attributed to the combination of a reduction in fluorophore number by $\mathrm{NaOH}$ attacking and an increase in dequenched molecule number. After 40 minutes of exposure to $\mathrm{NaOH}$, the intensity started to drop below the starting level, however, the precise quantification using the new method showed an over $80 \%$ fluorophore loss from the surfaces (black solid squares). As such, the new quantification method is demonstrated here to be reliable to asses the stability of functionalized surfaces. 


\section{Evaluation of Regeneration Methods}

Regeneration of functional groups on the surfaces allows to reuse the microchannel and the SMR devices many times. In this study, regeneration was done by the complete removal of the silane layer and restoration of hydroxyl groups. The fluorescence quantification method was applied to assess the success and efficiency of surface regeneration strategies for sequential experiments on the same microfluidic chip.

Plasma treatment, piranha and $0.1 \mathrm{~N} \mathrm{NaOH}$ were used respectively to regenerate the channel surfaces. Further functionalization resulted in regeneration of reactive amine groups that were quantified by fluorescence labeling with the standard mixture. The primany microchannels, which were packaged after production, were set as the reference. As shown in Figure 4.11, only about one third of the amine groups could be redeposited compared to the native surfaces, indicating that plasma treatment was not sufficient to remove all the deposited amine groups from previous functionalization. In contrast, the rinse with piranha and $0.1 \mathrm{~N} \mathrm{NaOH}$ solutions served to clean the surfaces of the used microchannels, and the amount of the regenerated functional amine groups were measured to be comparable to the reference. The surfaces cleaned with piranha solution were even redeposited with ca. $10 \mathrm{pmol} / \mathrm{mm}^{2}$ amine groups more than the reference surfaces, possibly due to the strong oxidation effect of the piranha solution. Although both of the reagents can be used alternatively for transparent microchannels, the use of piranha solution for SMR devices is more appropriate, since $\mathrm{NaOH}$ cleans the surfaces under an etching mechanism and can cause leaking problems of the suspended SMR resonators surrounded by vacuum.

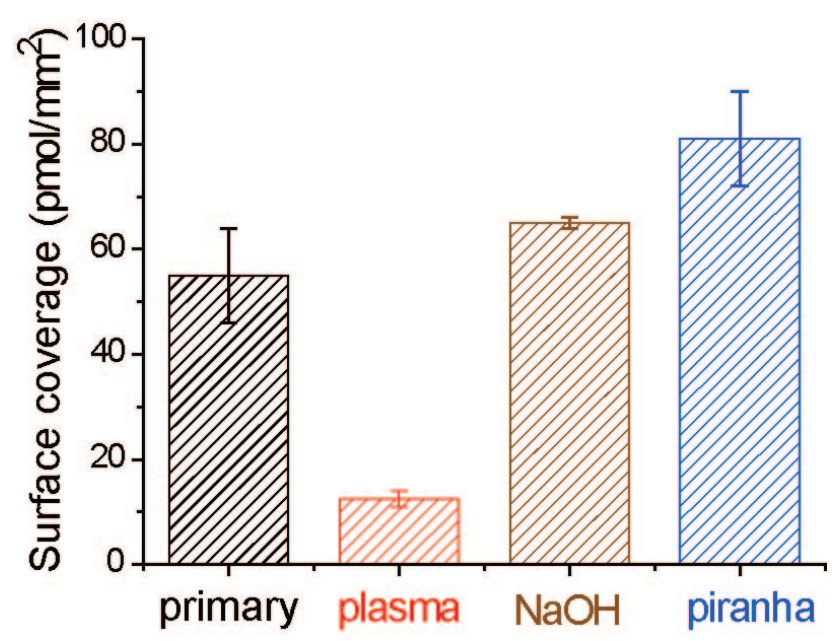

Figure 4.11: Regeneration of amine functionalized surfaces detected by fluorescence quantification. 


\subsubsection{Protein Binding Capacity of Amine-Functionalized Surfaces Using Glutaraldehyde Coupling}

GFP proteins (rAcGFP1) were used to evaluate the binding capacity of vapordeposited APTMS layers using glutaraldehyde coupling. Non-specific adsorption of GFP molecules to APTMS layers in the absense of a crosslinker was extremely high as shown in Figure 4.13 (I), indicating that the negatively charged GFP (pI 5.34 [166]) under physiological conditions could interact with the partially protonated amine groups on the channel surfaces. In contrast, the surface of a reference channel without aminosilane deposition, shown in Figure 4.13 (II), was prevented from non-specific binding by passivation with BSA.

Glutaraldehyde was chosen to functionalize the amine groups on the surfaces with aldehyde groups, as it has been reported to be one of the most efficient agents to generate thermally and chemically stable crosslinking [149, 174, 273]. However, care must be taken due to the high reactivity and the complexity in aqueous solution, because at least 13 possible polymeric forms under different conditions ( $\mathrm{pH}$, concentration, temperature, etc.) have been found in equilibrium, as partially shown in Figure 4.12 (I-VI) [174]. Different reaction mechanisms could govern the process of amine-aldehyde-reaction, as shown in Figure 4.12. In the SMR measurements, these large polymeric aggregates could easily block the microchannels during the injection and thereby impede further experiments. To address this problem, fresh glutaraldehyde solutions were centrifuged for at least 10 minutes at $4^{\circ} \mathrm{C}$ and only the supernatant containing maximal content of monomeric molecules was introduced to the microchannel surfaces.

The binding capacity of the GFP molecules to the APTMS-functionalized channel surfaces was quantified with fluorescence measurements in situ. The calibration showed the linear correlation between the fluorescence intensity and GFP concentrations in the microchannel (Figure 4.13 inset). Figure 4.13 (a, III) shows that GFP molecules bind efficiently to the glutaraldehyde-functionalized surfaces. The surface density of the immobilized GFP was calculated to be $1.2 \pm 0.5 \mathrm{pmol} / \mathrm{mm}^{2}$.

The stability of immobilized GFP molecules was tested by running buffer solutions inside the microchannels. The surfaces were exposed to the fluorescence illumination only while taking images with exposure time of one second. The plot of Figure 4.14 shows that ca. $90 \%$ of the initial intensity remained at the same level after 60 minutes rinsing. The $10 \%$ signal reduction might arise from the photo- 


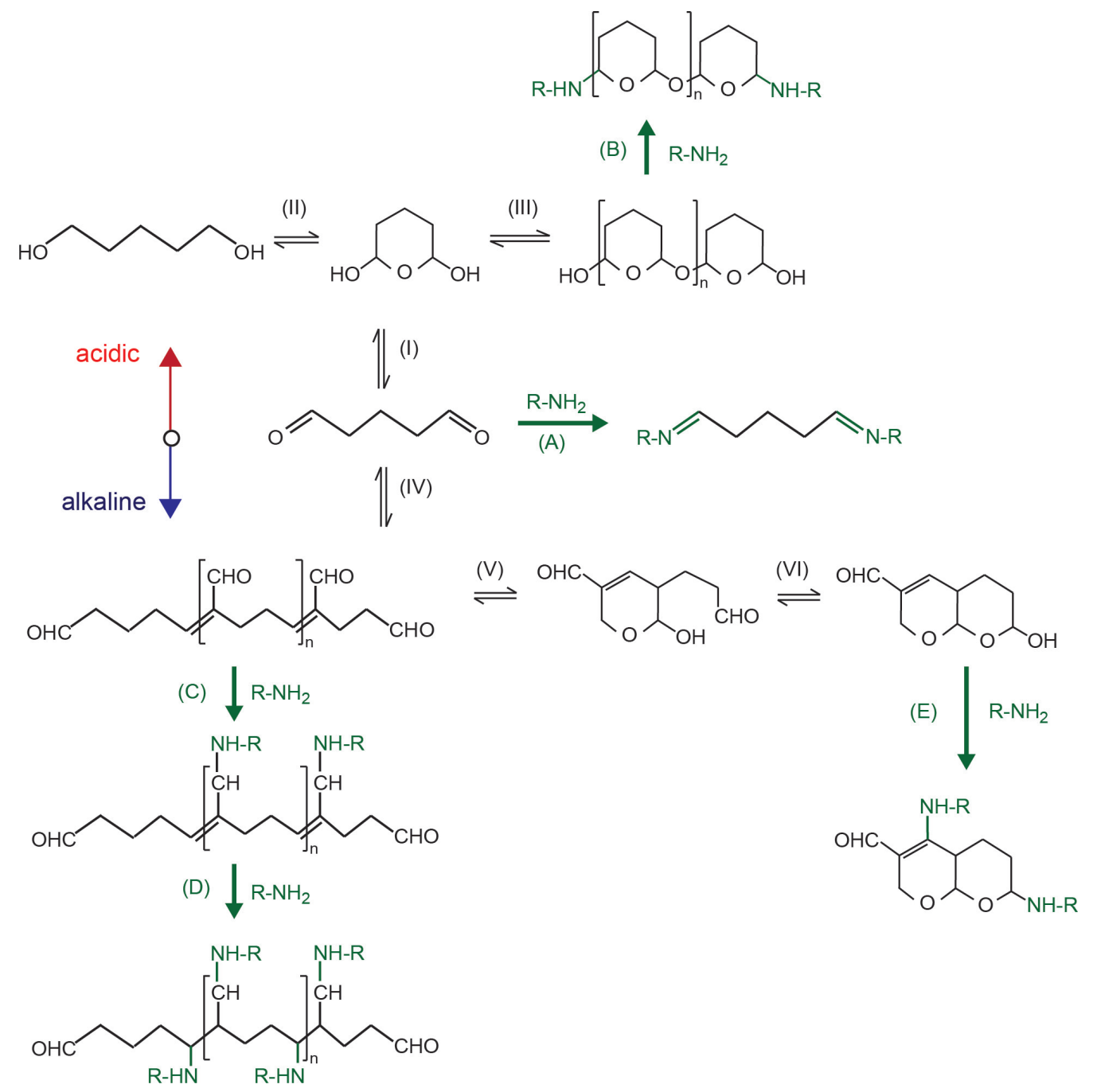

Figure 4.12: Schematic showing possible monomeric and polymeric structures (I-VI) of glutaraldehyde in aqueous solutions and the subsequent reaction mechanisms with amine groups of targeted proteins.

bleaching of the GFP molecules, since the signal after $16 \mathrm{~h}$ was kept at the same level with the signal detected at $1 \mathrm{~h}$. In comparison, $40 \%$ of the non-specifically adsorbed GFP molecules were rinsed off the surface within 5 minutes as shown in Figure 4.14 (black squares), clearly indicating the weak interaction between the GFP molecles and the surface amine groups. The results validated the stability of the immobilized GFP proteins by using glutaraldehyde as the coupling-agent. Based on this, the method can be reliably applied for immobilization of amyloid fibrils. 
(a)

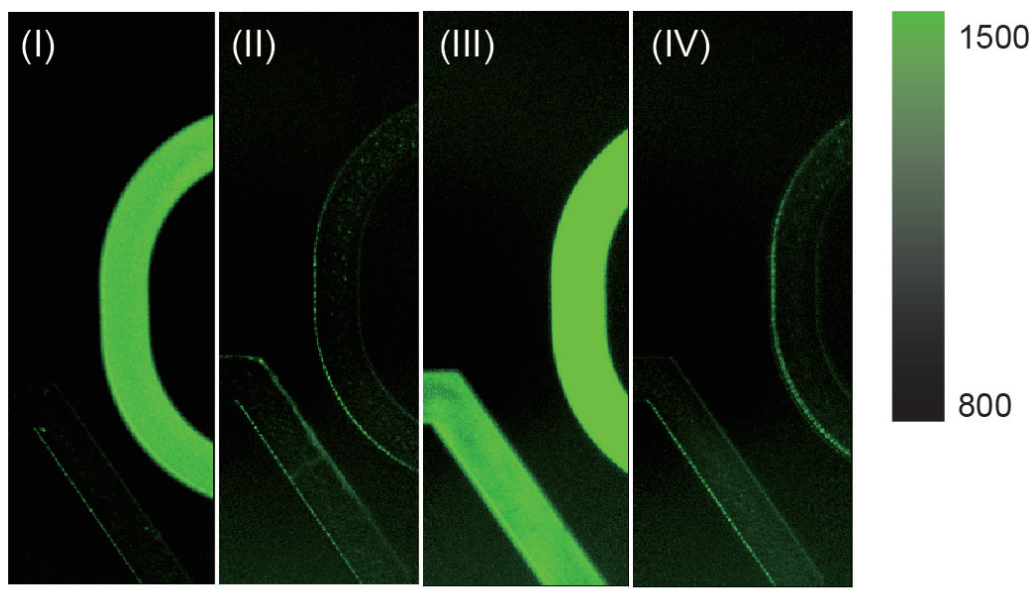

(b)

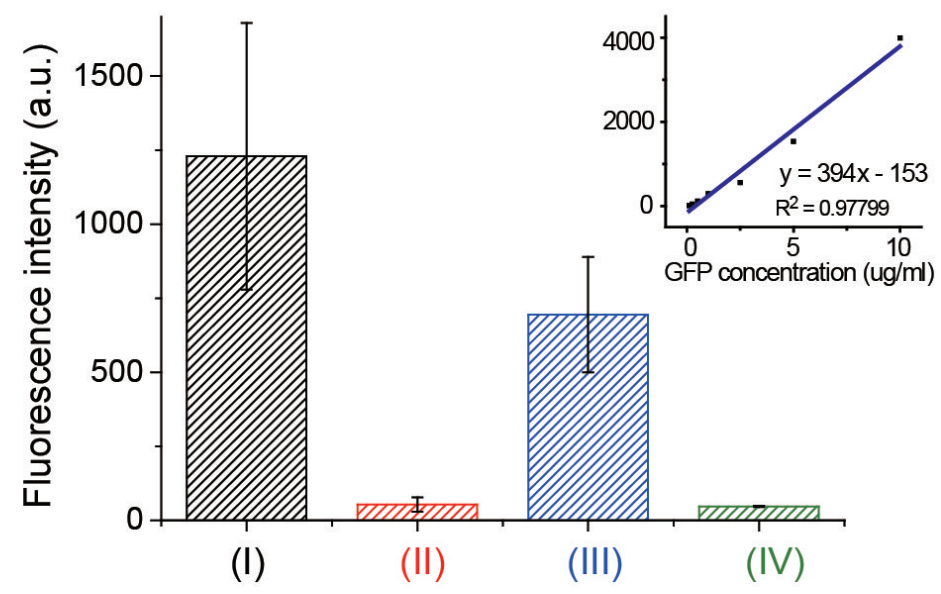

Figure 4.13: Fluorescence images (a) and intensity plot (b) of immobilized GFP on the APTMS-treated (I), BSA-passivated (II), APTMS and GA-treated (III), and APTMSGA-treated and ethanolamine-passivated (IV) surfaces. (Inset) Calibration of fluorescence intensity with various GFP concentrations.

\subsubsection{Surface Functionalization with Carboxymethyl Dextrans}

In this study, carboxymethyl(CM)-dextran was also used to further functionalize the APTMS-modified surfaces. CM-dextran of $150 \mathrm{kDa}$ molecular weight was deposited at $40 \mathrm{mg} / \mathrm{mL}$ in PBS buffer ( $\mathrm{pH}$ 8.0) in a mixture of EDC and NHS solutions. The initial motivation for using CM-dextran was to achieve higher capacity of immobilized proteins in the voluminous hydrogel matrix. As a biocompatible and hydrophillic polymer, dextran has been suggested to be a preferred linker for protein immobilization $[72,122,257,274]$. In addition, the anti-fouling material can also prevent non-specific binding. All these properties have been exploited to couple biomolecules in the field of Biacore chips and microarray systems [246]. Here, GFP was also used to assess the binding efficiency.

Two coupling protocols were tested on the CM-dextran modified surfaces, as 


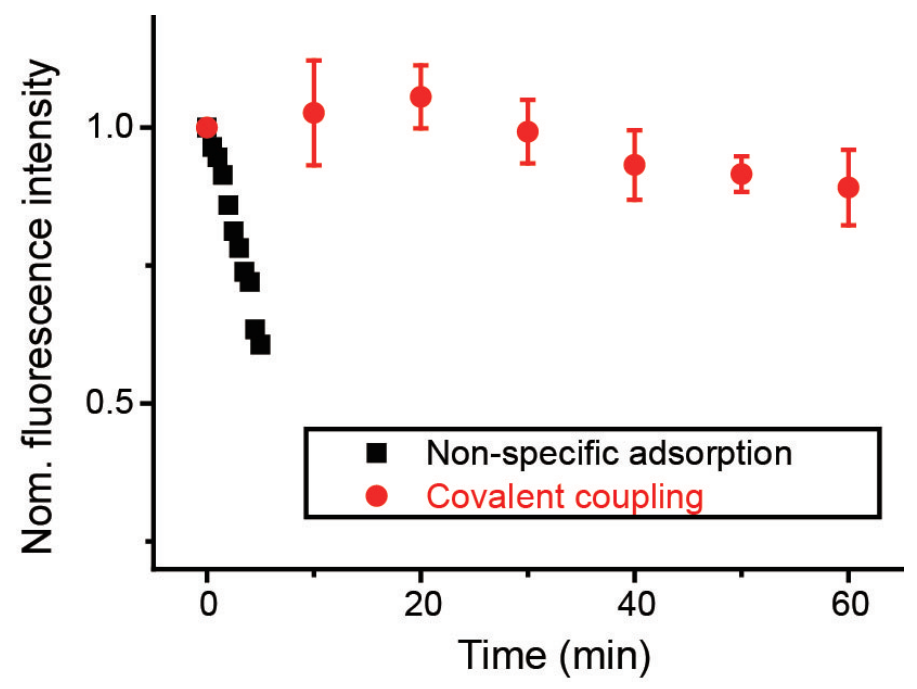

Figure 4.14: Plot showing the stability of non-specifically adsorbed GFP proteins (black squares) and covalently immobilized GFP proteins using glutaraldehyde (red dots) on the APTMS-modified surfaces.

shown in Figure 4.15:

- (I) CM-dextran that was commercially obtained was activated with EDC/NHS. Due to the voluminous structure of the matrix, the carboxyl groups only partially reacted with the surface amine groups. The remaining activated carboxyl groups were then used to link GFP molecules (protocol IA). Alternatively, sodium chloroacetate was used to further enrich the carboxyl groups of the dextran matrix by reacting with its hydroxyl groups (protocol I-B). The proteins were then coupled to the EDC/NHS-activated carboxyl groups in both protocols.

- (II) After the deposition of CM-dextran on the channel surfaces, sodium periodate was used to direct the oxidation of the cyclic dextran ring to dialdehyde groups, which could form Schiff's base with amine groups of the proteins. Further reduction using sodium cyanoborohydride stabilized the coupling.

The binding of GFP proteins to the CM-dextran surfaces was generally not as efficient as expected. As shown in Figure 4.16, the intensity of channel "L" following method (I-A) of image (I-A) was much lower than channel "R" adsorbed with GFP non-specifically and channel "L" of image (II) where GFP was coupled to the aldehyde groups generated using glutaraldehyde. The low signal indicates that not many carboxyl groups of CM-dextran were left for GFP coupling. In channel "M" of image (I-B), higher intensity was expected since the use of sodium chloroacetate with method (I-B) could theoretically generate more carboxyl groups. However, only comparable intensity to the untreated CM-dextran layer was measured in this 


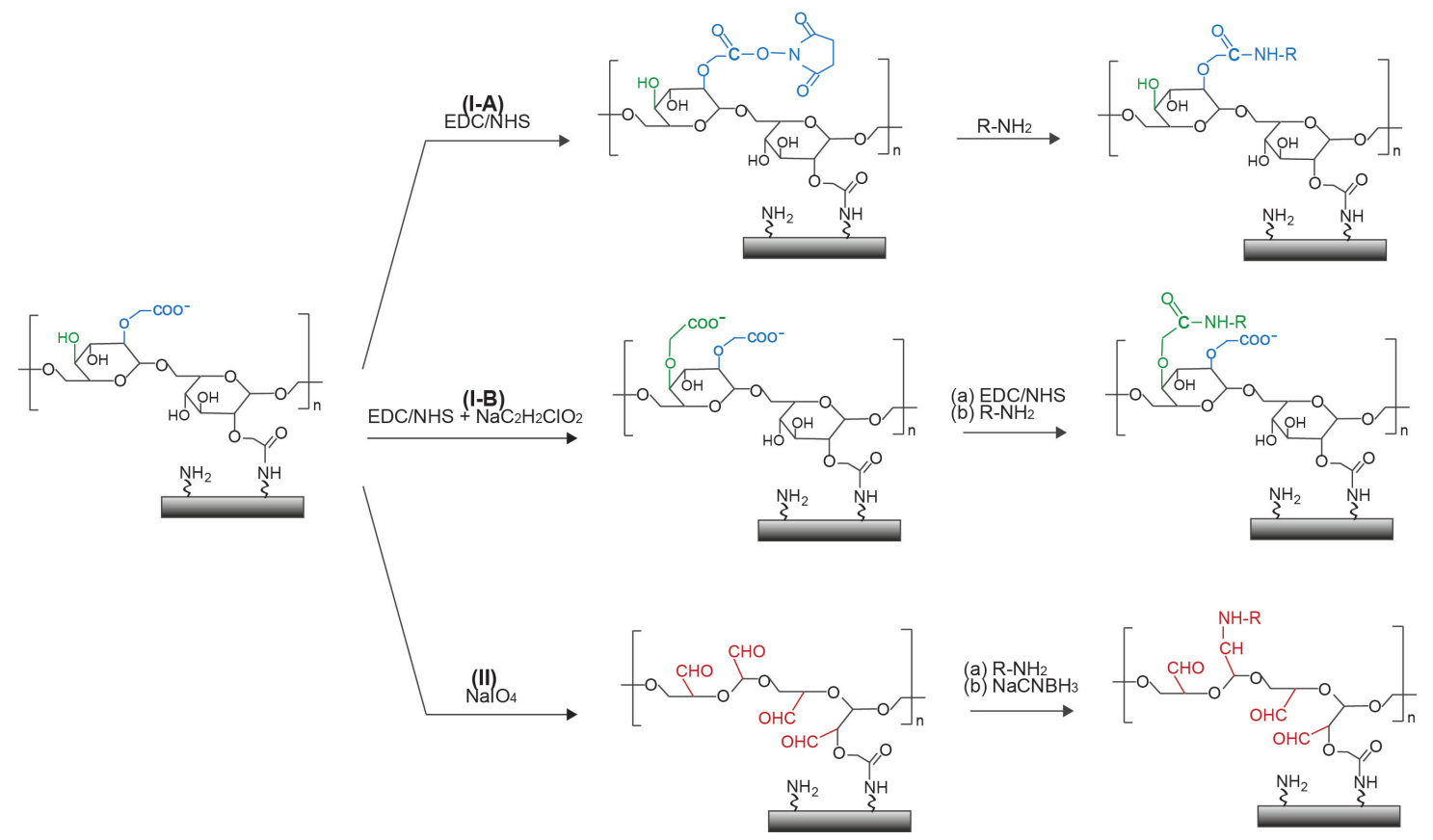

Figure 4.15: Schematics for immobilization of GFP proteins (presented as $\mathrm{R}-\mathrm{NH}_{2}$ ) to the carboxymethyl-dextran hydrogels on the microchannel surfaces.

channel. In channel "M" of the image (II), the low fluorescence intensity did not show efficient GFP binding to the CM-dextran modified surface that was oxidized by sodium periodate. The signal in channel " $\mathrm{M}$ " was at the background level when GFP was bound to aldehyde groups generated by using glutaraldehyde in the channel "L" of image (II). In general, despite the many advantages of protein immobilization on the biomimetic dextran-modified surfaces, more studies are required to develop a reliable and consistent way for higher binding capacity and efficiency.

\subsubsection{Immobilization of Insulin Fibrils}

So far, the development and the characterization of in-channel surface functionalization have been presented here for reliable protein immobilization. The techniques can now be applied to the primary objective of this study, the immobilization of amyloid fibrils. Since the last two decades, insulin aggregation has been intensively studied since the amyloid-like insulin fibrils have been found to be associated with diseases such as injection amyloidosis. Moreover, insulin fibrillation occurs frequently during insulin production and long-term storage $[25,63,116]$. Due to the simplicity of insulin monomers that contain two short polypeptide chains with one intramolecular and two intermolecular disulfide bonds, as shown in Figure 4.17, 
CM-dextran + EDC/NHS:
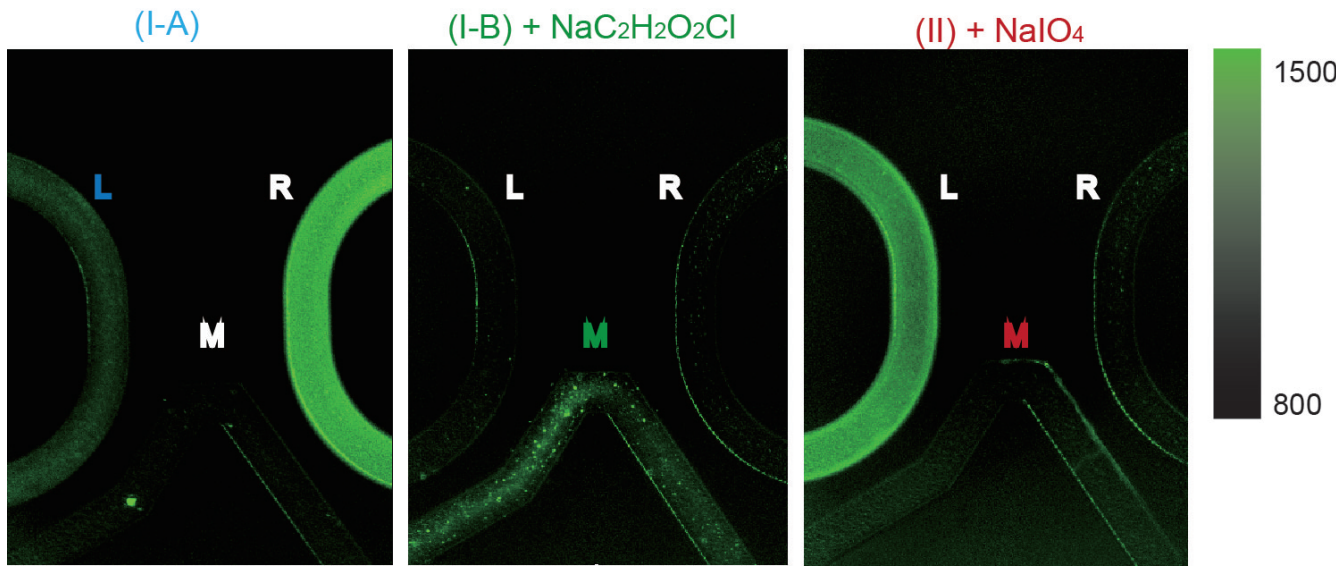

Figure 4.16: Fluorescence images of immobilized GFP proteins in APTMS-modified microchannels using CM-dextran alone (I-A, channel "L"), CM-dextran and sodium chloroacetate (I-B, channel "M"), glutaraldehyde (II, channel "L"), CM-dextran and sodium periodate (II, channel "M"), BSA as reference channels (I-A, channel "M"; I-B, channel "L" and "R"; II, channel "R"). The channel "R" in Image (I-A) shows non-specific adsorption of GFP on APTMS-modified channel surfaces.

many studies have been carried out and in vitro fibril formation is relatively easy to achieve and control. However, in microfluidic systems, optimal conditions need to be established to produce fibrils ideally with homogeneous size distribution. Furthermore, the biofunctional properties of the immobilized insulin molecules on the microchannel surface need to be retained.

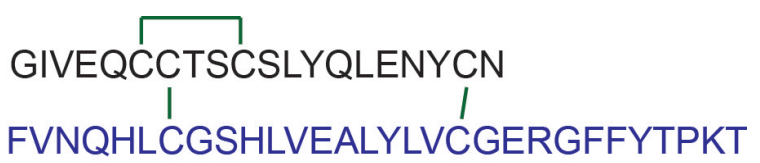

Figure 4.17: Insulin structure with two polypeptide chains.

\section{Size Distribution of Insulin Fibrils}

Preparation of insulin seed fibrils with uniform size distribution is of great importance for the kinetic measurements of the fibril growth, since the resultant average growth rates are representative for a defined ensemble of fibrils. Furthermore, the uniform seed fibril length can be used to estimate the number concentrations of the fibrils immobilized on the surface of the SMR microchannel (Section 5.2.1). Rate constants can thereby be accurately determined as the number of monomers binding to the fibril ends per time. 
In many protocols used to prepare insulin fibrils in vitro, monomers were dissolved in $\mathrm{HCl}$ solution $(10 \mathrm{mM})$ at $\mathrm{pH} 2$ and with high salt concentrations $[25,38,107,120]$ or glycine buffers at pH 2 [9]. Since glycine contains amine groups in its structure and will compete with insulin fibrils to react with the surface aldehyde groups, it cannot be used as the buffer solution for immobilization purposes. Initially, insulin aggregation was done in $25 \mathrm{mM} \mathrm{HCl}$ solution $(\mathrm{pH} 1.6$, $100 \mathrm{mM} \mathrm{NaCl}$ ) at $37^{\circ} \mathrm{C}$ for more than two days until the aggregation was complete as validated with the ThT assay. The solutions turned cloudy with fibrils, and electron microcopy showed that long fibrils clumped into large aggregates, as shown in Figure 4.18 (b). These large clumps could easily clog the microchannels and therefore are not applicable to the detection systems. This problem was solved by dissolving the insulin monomer in $10 \mathrm{mM}$ HEPES at $\mathrm{pH} \mathrm{2.0.} \mathrm{The} \mathrm{resultant} \mathrm{long}$ fibrils in Figure 4.18 (c) were dispersed in the solution which looked clear even after the aggregation was done. Furthermore, ultrasonication was used to break down the preformed fibrils into short fibrils, as the approach has been reported to produce monodispersed fibrils of minimum size [42]. In this thesis, preformed long fibrils were ultrasonicated for 150 minutes at $4^{\circ} \mathrm{C}$. The obtained short seed fibrils were then filtered with a $0.22 \mu \mathrm{m}$ membrane filter. $70.3 \pm 11.5 \%$ seed fibrils were retained in the filtrate for immobilization. Using EM (Figure 4.18), the average length of the obtained fibrils was evaluated to be $97.6 \pm 20.1 \mathrm{~nm}$ and the average width to be $12.3 \pm 0.8 \mathrm{~nm}$. The width value implies that a mature fibril consists of two protofibrils that are ca. 5-7 nm wide $[1,119]$. As the mass-per-length value of the fibrils with two protofibrils has been determined to be $2.85 \pm 0.35 \mathrm{kDa} / \AA[43,116]$, the average buoyant mass of a single short fibril was calculated to be $1.2 \pm 0.4 \mathrm{ag}$.

\section{Immobilized Insulin Fibrils}

The solutions of short insulin fibrils were immobilized to the microchannel surfaces following aminosilane and glutaraldehyde functionalization and stabilization. The immobilized fibrils were then labeled with ThT molecules to allow imaging by fluorescence microscopy. In the small $2 \times 2 \mu \mathrm{m}$ microchannel and the right bypass channel shown in Figure 4.19, the short insulin fibrils were observed to be homogeneously distributed on the surfaces. The comparable fluorescence intensities in both channels indicate that there was no difference in binding efficiency in both channels. The flow of the fibril solution was unobstructed, and the flow direction could be controlled without clogging. In the left bypass channel, the surface was passivated by coupling ethanolamine instead of insulin fibrils to the reactive aldehyde groups. After ThT solutions were filled in the channel followed by rinsing 

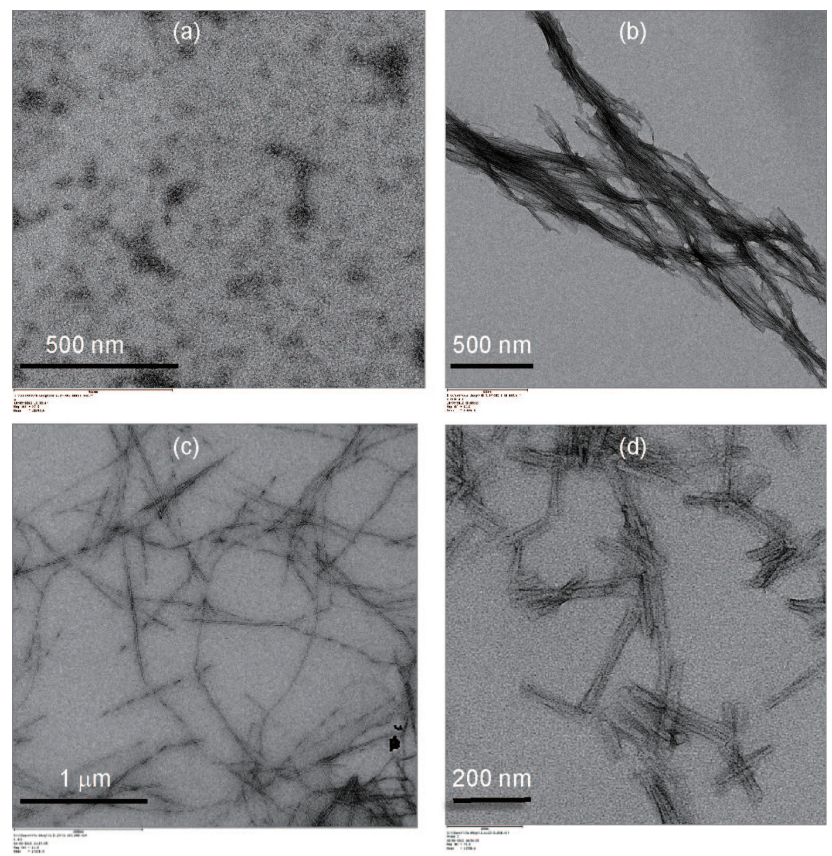

Figure 4.18: Electron micrographs of (a) insulin monomers, (b) insulin fibrils grown in $\mathrm{HCl}-\mathrm{NaCl}$ solution, (c) insulin fibrils grown in HEPES solution and (d) sonicated insulin fibrils.

with the buffer solution, the fluorescence intensity was kept at background level, indicating efficient prevention of the non-specific fibril adsorption.

(a)

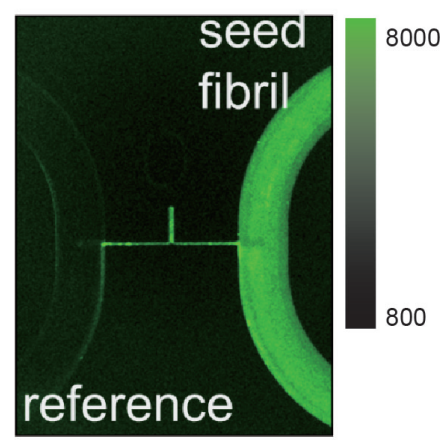

(b)

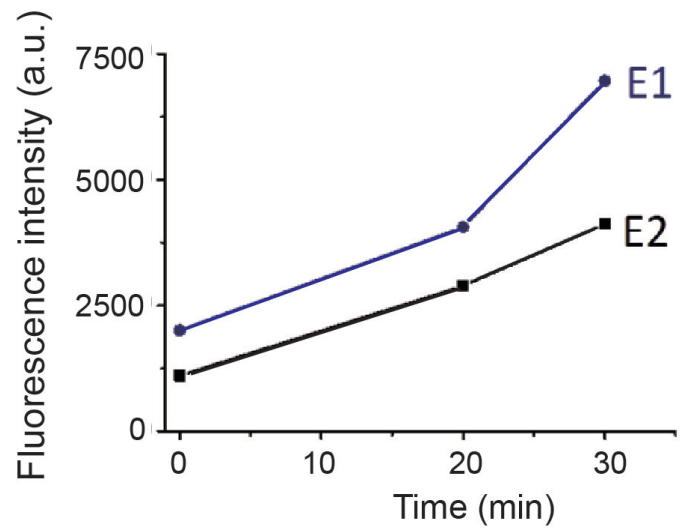

Figure 4.19: Fluorescence image of immobilized insulin seed fibrils inside the small microchannel and the right bypass channel (a). The left bypass channel modified with APTMS and glutaraldehyde was passivated from non-specific binding using ethanolamine. Plot (b) shows the intensity increase detected by labeling with ThT compound when the seed fibrils grow in the presence of monomers.

To be sure that the immobilization on the surface did not fundamentally change the structure of the fibrils and affect amyloid growth, monomer insulin solutions were introduced to the immobilized fibrils and the grown fibrils were labeled 
again with ThT to give a detectable fluorescence signal. As plotted in Figure 4.19 (b), both experiment replicas show that the immobilized fibrils were able to grow, indicating that the functionality of the fibrils was preserved. However, as discussed in Section 4.2, quantitative interpretation of the growth kinetics is impossible by fluorescence detection using ThT-binding assay due to the unknown binding stoichiometry and the risk of interfering with the aggregation process.

The covalent protein immobilization approach developed in the transparent microchannel systems using fluorescence microscopy can be readily applied to the SMR detection system. The stability and reproducibility tests of this approach is presented and discussed in the next chapter. 


\section{Chapter 5}

\section{Kinetic Measurements of Amyloid Fibril Growth using SMR}

\subsection{Introduction}

Having established protein immobilization techniques using transparent microchannels and fluorescence microscopy, the methods were applied to the SMR detection system for the precise kinetic measurements of amyloid growth. The study initially focused on the elongation process using the proteins $\alpha \mathrm{S}$, that is closely related to Parkinson's disease, and insulin that is relevant for diabetes patients. In this chapter, achievements with the novel surface-binding-based SMR approach in characterization of fibril immobilization density, precise measurements of elongation kinetics, studies on the underlying mechanisms, thermodynamics and effects of environmental factors, as well as screening for potential inhibitors are presented.

In the first two parts of this chapter, the feasibility and the reliability of the kinetic measurements were tested, making use of both electrostatic and covalent immobilization techniques. Nonspecific binding, stability of immobilized seed fibrils, and reproducibility were evaluated and compared using both immobilization methods. The most reliable method was selected and applied for further accurate quantitative measurements of elongation kinetics.

Subsequently, elongation kinetics were studied using insulin as a model system for the fibrillation process. Insulin has a very strong propensity to form fibrils and its amyloid deposits have been found in patients with type II diabetes after continuous insulin infusion and after repeated insulin injections. In addition, insulin aggregation is a serious problem in the protein production, storage and delivery processes [188]. It has been frequently reported that low $\mathrm{pH}$ of $1-3$ in the insulin 
purification steps and agitation during transportation can trigger aggregation. Due to the similarity of amyloid fibrils formed by different proteins, the results are of significance for many systems other than insulin.

As presented in the previous Section 1.1.2, there is significant experimental evidence demonstrating that the fibril elongation process is a bimolecular reaction $[39,105,138,146,197]$. The elongation rate $\frac{d N}{d t}$ can therefore be described by Equation 5.1 with the number $N$ of monomer molecules bound to the fibrils, the absolute rate constant $k$, the number of the fibrils immobilized on the surface $N_{\text {Fibril }}$ and monomer concentration in solution $c_{\text {Monomer }}$.

$$
\frac{d N}{d t}=k * N_{\text {Fibril }} * c_{\text {Monomer }}
$$

The SMR measurements allow determination of the elongation rates and of the absolute rate constants, which are fundamental characteristics of the process. As discussed in Section 1.2, the kinetics of amyloid growth is conventionally measured in solution and depends strongly on the presence of seed fibrils, concentration of monomers and whether the solutions are stirred [146]. Quantitative interpretation of the results is extremely challenging, as the concentrations of fibrils are unknown and the absolute growth rates therefore cannot be evaluated. In the surface-based SMR measurements, the precise determination of the kinetics at various concentrations and temperatures enables deeper understanding of the mechanisms and the thermodynamics of the explored elongation process.

Here, the surface-based SMR approach is also demonstrated to be a reliable label-free inhibitor screening method for amyloid growth. Importantly, the SMR measurements can also be used to screen for fibril dissociation by monitoring degradation of surface fibrils in the presence of chemical compounds or at various $\mathrm{pH}$ and temperature. Based on both applications, the SMR measurements could be of great therapeutic and preventive importance.

\subsubsection{Sensitivity of Surface-Based SMR Measurements}

The correlation between the subtle change in the resonator mass and the frequency shift was determined by measuring the frequency shifts of sodium chloride solutions at different concentrations $(2-20 \mathrm{mM})$. The density variation of the fluids arises from dissolving different amounts of $\mathrm{NaCl}$ in water and was estimated for different salt concentrations using a table of fluid density vs. NaCl concentration [159]. The 
slope of the linear fit in Figure 5.1 shows that a density change of $1 \mathrm{mg} / \mathrm{cm}^{3}$ resulted in a frequency shift of $76.11 \pm 1.19 \mathrm{~Hz}$. Based on Equation 2.11 in Section 2.1.1, the density sensitivity $S_{\rho}$ was determined to be $53979 \pm 844 \mathrm{ppm} /\left(\mathrm{g} \cdot \mathrm{cm}^{-3}\right)$ by using a torsional SMR containing a $3 \mu \mathrm{m}$ high and $8 \mu \mathrm{m}$ wide channel and having a resonance frequency of ca. $1.41 \mathrm{MHz}$. For surface-based measurements with this device, $1 \mathrm{~Hz}$ frequency shift was correlated to be a change in surface density of $1.43 \pm 0.02 \mathrm{ng} / \mathrm{cm}^{2}$ (Equation 2.11 in Section 2.1.1). This value was later used to estimate the density of amyloid seed fibrils immobilized on the channel surfaces.

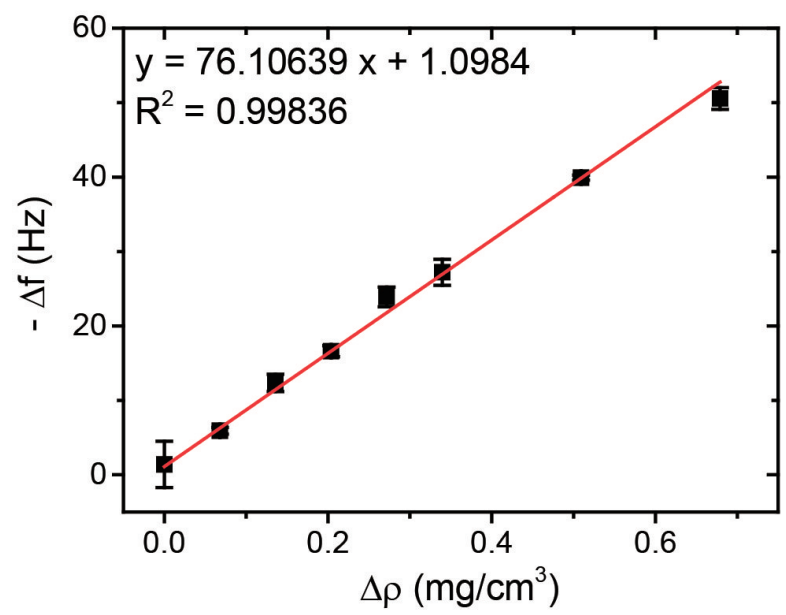

Figure 5.1: Calibration of the resonance frequency shift as a function of fluid density.

\subsubsection{Comparison of Reproducibility and Stability Using Electrostatic and Covalent Immobilization Techniques}

In this study, accurate determination of elongation rates requires stable seed fibril immobilization on the microchannel surface, providing known and consistent fibril densities throughout the measurements.

In the SMR measurements, the PLL-based electrostatic immobilization technique was initially applied and the growth of the adsorbed $\alpha$ S-fibrils on the microchannel surface was monitored when they were exposed to monomer solutions. A frequency decrease in a frequency-time-plot indicates a mass increase of the resonator as a result of molecules attaching to the microchannel surface. As shown in Figure 5.2(a), $146 \pm 74 \mathrm{~nm}$-long fragmented $\alpha \mathrm{S}$ seed fibrils, as imaged using EM (Figure 4.2 (c) in Section 4.2), were immobilized onto the PLL-coated surface by following a procedure similar to the one applied in the transparent 
microchannels. This resulted in a frequency decrease of ca. $25 \mathrm{~Hz}$, corresponding to a fibril surface density of $38.6 \pm 0.5 \mathrm{ng} / \mathrm{cm}^{2}$. The immobilized fibrils were then brought into contact with a running monomer solution $(20 \mu \mathrm{M}$ in HEPES buffer at $\mathrm{pH}$ 6.5) for 30 minutes. The frequency decreased continuously, indicating fibril growth in the presence of monomer molecules (Figure 5.2 (a) and (b, blue line)). This was also the first proof-of-principal that the surface-based SMR measurements could be applied to monitor amyloid elongation. By measuring the frequency difference under buffer before and after the injection of monomer solutions over time, the average growth rates can be estimated as total amount of mass increase per time.

A significant disadvantage of the PLL-based immobilization is the high level of non-specific binding of $\alpha \mathrm{S}$ monomers. This limits the application of the method for further accurate kinetic measurements. As plotted in Figure 5.2 (b, black line), a frequency decrease of $19 \mathrm{~Hz}$ clearly indicates that monomer molecules attach to the channel surfaces in the absence of seed fibrils. In comparison, 30 minutes flow of the monomer solution over the fibril surfaces resulted in a frequency decrease of $65 \mathrm{~Hz}$ (Figure 5.2, plot a and b with blue line). Although the frequency decrease due to fibril elongation is over three times larger than that resulting from non-specific adsorption, it is difficult to distinguish the proportion of the non-specific signal from the elongation signal. The results can therefore not be used to quantify the elongation on-rates.

Moreover, as the monomer concentration was kept constant by continuous flow, the fibril growth was expected to be at a constant rate according to the fibril elongation kinetics presented in Equation 5.1. However, a non-linear growth of the fibrils was observed here. The non-linear growth could arise from the combined effect of elongation and the continuous loss of the immobilized fibrils off the surface due to the unstable immobilization, although this instability was not recognized in the detection with the transparent microchannel system presented in Section 4.2.

To reduce the non-specific-binding of monomers onto the surface, PLL-graftedCM-dextran was deposited in the SMR measurements. The subsequent seed fibril immobilization was done by using EDC/NHS-activator as introduced in Section 2.2.1. Using this immobilization approach, non-specific binding of $\alpha \mathrm{S}$ monomers was efficiently suppressed due to the hydrophilicity of the dextran matrix as shown in Figure 5.2 (c, black line). A frequency shift of only $19 \mathrm{~Hz}$ after 30 minutes in the presence of $20 \mu \mathrm{M}$ monomer solution (c, blue line) showed that the polymeric dextran matrix did not increase the surface density of the seed fibrils for monomer 
(a)
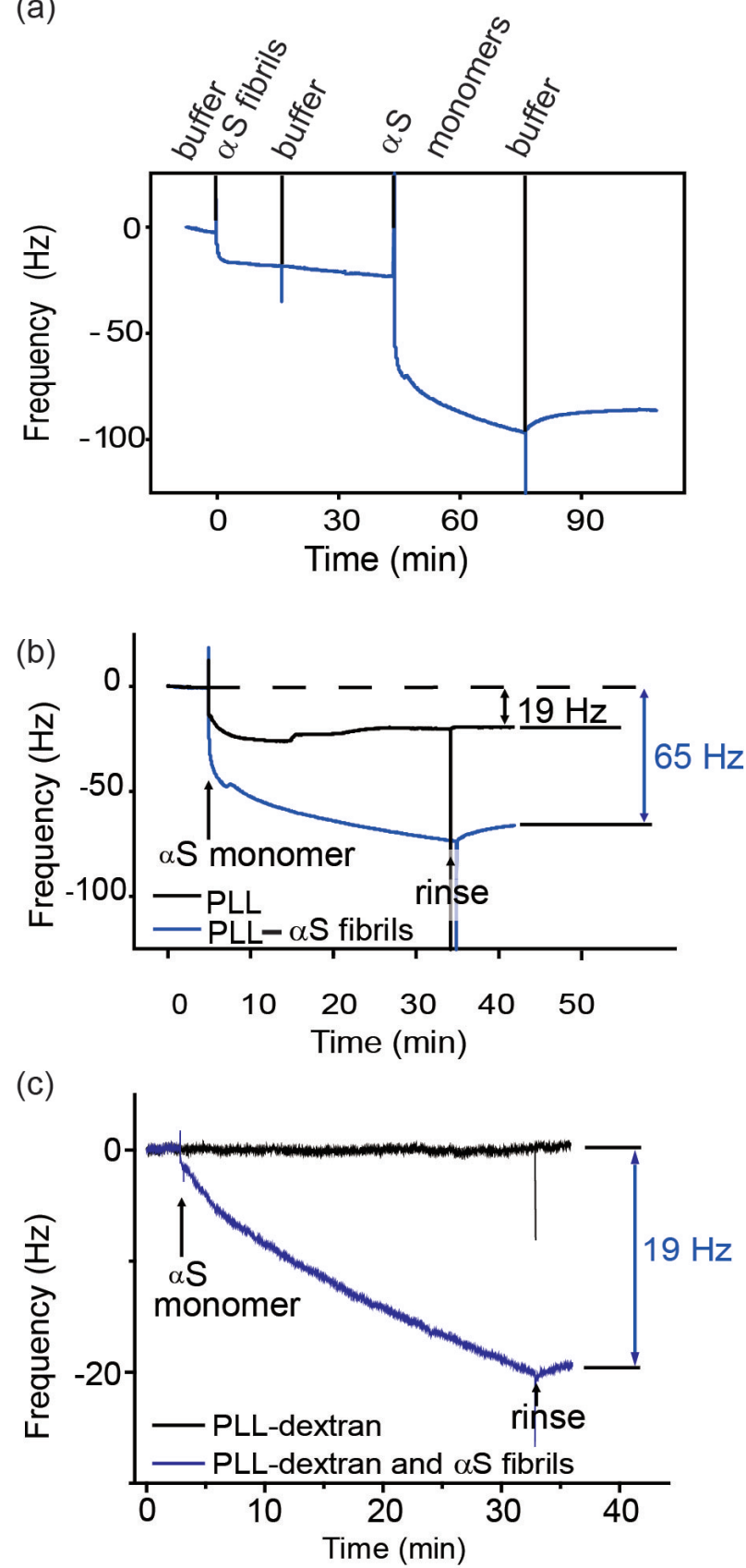

Figure 5.2: SMR measurements of binding of $\alpha \mathrm{S}$ monomers to PLL-coated surfaces or PLL-immobilized fibrils (a); non-specific monomer adsorption (b); and binding of $\alpha \mathrm{S}$ to the surface using PLL-grafted-CM-dextran modification and EDC/NHS activation (c).

addition. Similar to the elongation monitoring on PLL-coated surfaces, the non-linear fibril growth over the extended experiment time is again indicative for unstable fibril immobilization on the surfaces.

To confirm that the observed non-linear growth of the fibrils immobilized using PLL and PLL-grafted-CM-dextran was caused by the unstable immobilization, the 
sequence of elongation measurements with three $\alpha \mathrm{S}$ monomer concentrations $(0.3$, 1 and $30 \mathrm{mg} / \mathrm{mL}$ ) was repeated multiple times and the resulting frequency shifts were compared. As shown in Figure 5.3 (a), the reproducibility experiment of the PLL-linked fibril elongation shows an approximately $30 \%$ systematic reduction of the frequency signals from experiment sequence E1 to E4, indicating that fewer fibrils were available for the elongation compared to the previous measurement under the same condition.

A similar experiment was conducted with the $\alpha \mathrm{S}$ fibrils immobilized using PLL-grafted-CM-dextran. By repeating the concentration sequence, a continuous fibril loss from the surface was also revealed, as shown in Figure 5.3 (b). As such, it is concluded that the electrostatic immobilization technique is generally not sufficient for the purpose of accurate kinetic measurements.

In comparison to the electrostatic immobilization, the stability of insulin fibril immobilization was also tested using the established covalent immobilization techniques with the aminosilane APTMS and glutaraldehyde as coupling agents. Here, three insulin monomer solutions $(0.6,2$ and $6 \mathrm{mg} / \mathrm{mL})$ were injected into the resonator microchannel in sequence for 5 minutes respectively. By repeating the experiment set for two more times, the frequency decrease as a result of monomer binding to the immobilized fibrils was unchanged. This is clearly shown in Figure 5.3 as the time courses of the frequency signals overlapped with one another. As such, it can be concluded that the insulin seed fibrils were stably immobilized on the surfaces over the extended experiment time (ca. $2 \mathrm{~h}$ ).

The comparison of the three reproducibility tests using different protein immobilization techniques demonstrates that only covalently immobilized fibrils are stable on the surface under continuous flow conditions and can thus be reliably applied in the SMR measurements for the quantitative kinetic studies.

In addition to the reproducibility tests, the long-term stability was verified by monitoring the growth rates of the same ensemble of the immobilized fibrils with the monomer solutions under the same condition $\left(0.4 \mathrm{mg} / \mathrm{mL}, 37^{\circ} \mathrm{C}\right)$ for one week. The plotted results, presented in Figure 5.4, show comparable rates over time and corroborate the stable immobilization of the fibrils. The small variation was possibly due to the small concentration variation of the freshly prepared monomer solutions.

Since both reproducibility and stability tests confirmed the reliability of the 

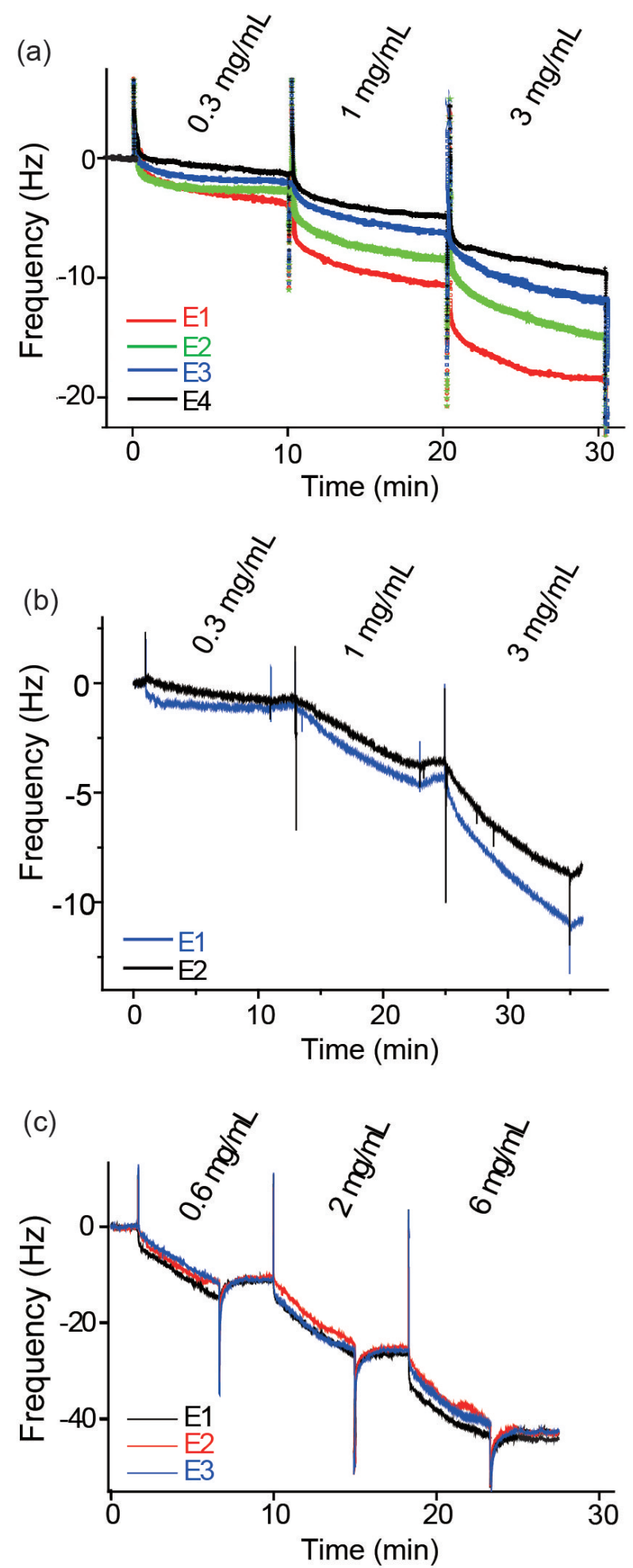

Figure 5.3: Reproducibility of the fibril elongation measurements by SMR using different immobilization techniques with PLL (a), PLL-grafted-CM-dextran (b) and aminosilane/glutaraldehyde (c).

SMR measurements, the detection system is demonstrated to be applicable for 


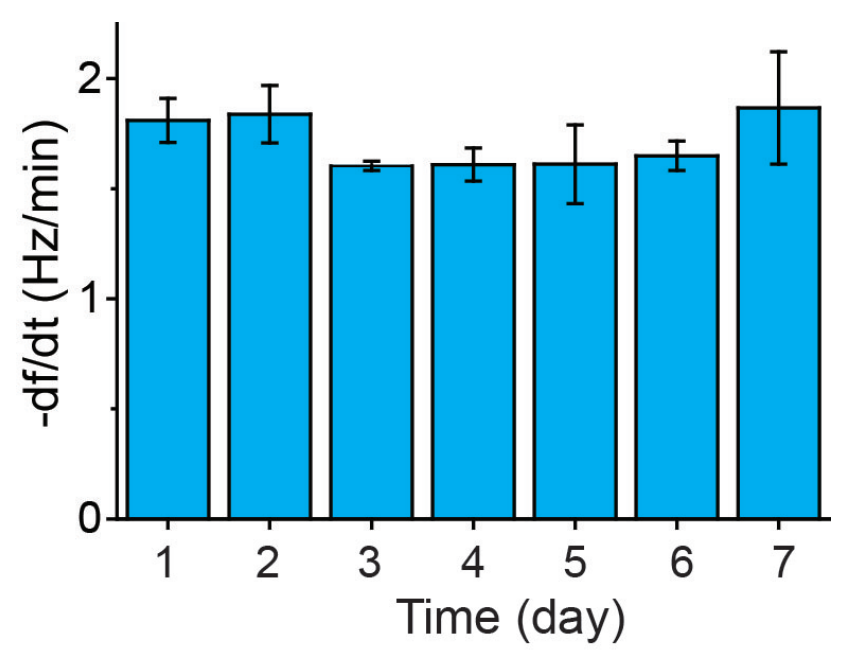

Figure 5.4: Stability test by measuring fibril growth with $0.4 \mathrm{mg} / \mathrm{mL}$ monomer solution at $37^{\circ} \mathrm{C}$ for one week.

quantitative measurements with many different conditions.

\subsection{Elongation Kinetics of Covalently Immobilized Insulin Fibrils}

\subsubsection{Surface Density of Immobilized Insulin Seed Fibrils}

Surface densities of covalently immobilized insulin seed fibrils were quantified in situ by measuring the frequency shift before and after the fibril solutions were introduced to the functionalized channel surface. The estimated quantities were the basis for determination of the elongation rates and the rate constants. In the SMR measurements, the immobilization steps that started with the aldehyde-containing surfaces were recorded, as shown in Figure 5.5. The fast solution change from water to the HEPES buffer ( $\mathrm{pH} 2.0)$ resulted in a sudden frequency decrease due to a density increase. The low $\mathrm{pH}$ running buffer was used here to retain the same $\mathrm{pH}$ environment of the seed fibrils and to avoid possible fibril precipitation by passing over the isoelectric point at $\mathrm{pH} 5.5$ [89]. Insulin seed fibrils were then introduced to the microchannel surface and the fibrils saturated the surface within 15 minutes. By comparing the signals with water before and after the seed fibril immobilization, a frequency decrease of $71.3 \pm 8.6 \mathrm{~Hz}$ corresponded to a fibril density of $734 \pm 89$ molecules $/ \mu m^{2}$. Here, the average mass of a single fibril was estimated to be $1.2 \pm 0.4$ ag (mass-per-length: $2.85 \pm 0.35 \mathrm{kDa} / \AA[116]$ ), 
characterized by EM in Section 4.3.2. The fibril-fibril distance was therefore determined as $37 \pm 13 \mathrm{~nm}$. The surface of the SMR chip was regenerated and the fibril immobilization was repeated at least four times. The error of regenerated surface densities of the insulin fibrils was ca. 10\%, indicating that the developed immobilization technique was highly reproducible.

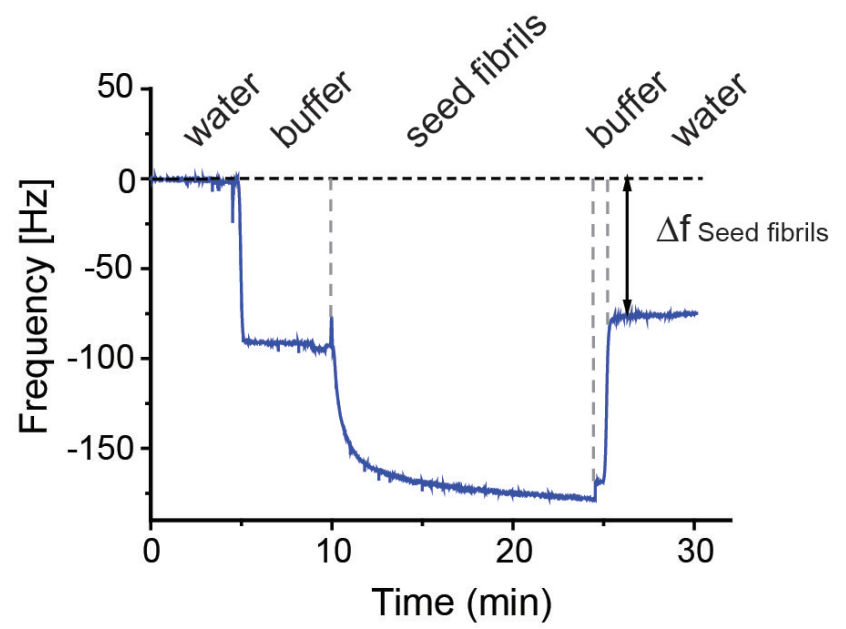

Figure 5.5: Immobilization of insulin seed fibrils on SMR microchannel surfaces.

To reconfirm the surface density of immobilized seed fibrils on the channel surfaces, open-surface samples were prepared with silicon substrates and detected by AFM. There were two differences in immobilizing the fibrils: first, the temperature was kept at $25^{\circ} \mathrm{C}$ instead of $37^{\circ} \mathrm{C}$; Secondly, open surfaces were incubated in solutions of fibrils and coupling agents with defined volume under stirring. However, the obtained surface densities should be comparable, as all the concentrations were sufficient to fully saturate the surface area of the substrates. Densely immobilized fibrils were shown in the AFM image (b) of Figure 5.6. An aminosilane-modified Si-substrate without fibril immobilization is shown for comparison in image (a). By counting the fibril peaks in the profile of image (c) along the drawn $2 \mu \mathrm{m}$-long line, the fibril-fibril distance was determined to be $46 \pm 7 \mathrm{~nm}$, which is consistent with the value obtained by SMR measurements.

\subsubsection{Determination of Absolute Rates of Insulin Fibril Elongation by SMR}

The elongation kinetics of covalently immobilized insulin seed fibrils were measured when the resonator microchannel was filled with a fresh monomer solution $(1 \mathrm{mg} / \mathrm{mL}$, glycine buffer, $\mathrm{pH} 2.0$ ) at $37^{\circ} \mathrm{C}$. The linear signal decrease in Figure 5.7 (red line) 
(a)

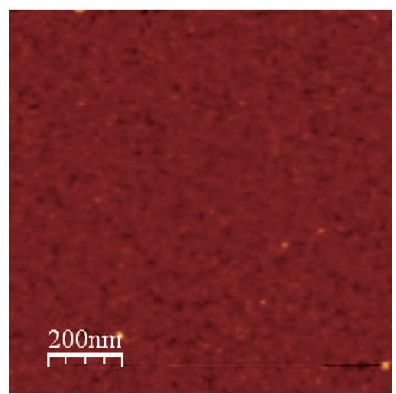

(b)

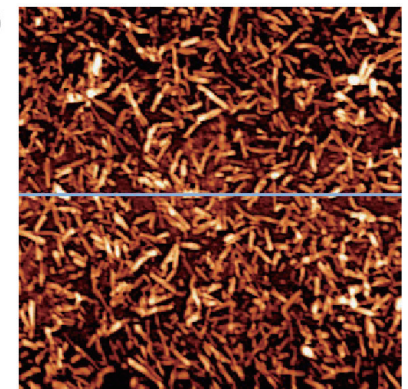

(c)

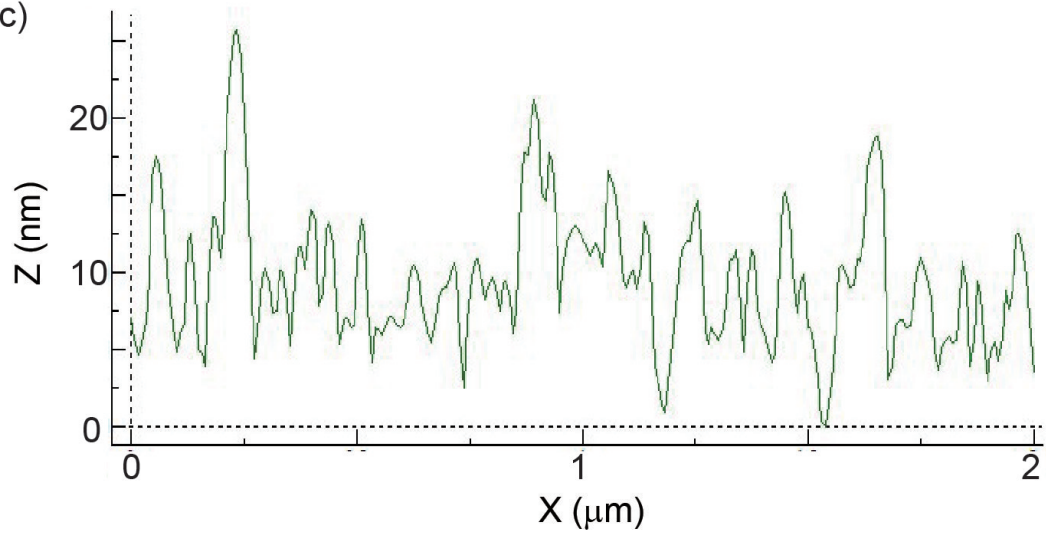

Figure 5.6: AFM images of APTMS-modified Si-substrate (a) and immobilized insulin seed fibrils on the Si-substrate surface (b). (c) Profile showing topography of fibrildeposited Si-substrate along the line drawn in (b).

over time indicates that the fibrils grow at a constant rate, as expected with the expression in Equation 5.1. Reference experiments were taken to test the non-specific adsorption of monomers to the passivated channel surfaces. The channel surfaces were first functionalized with aminosilane and glutaraldehyde, and glycine molecules instead of seed fibrils were then introduced to couple with the reactive aldehyde groups. As shown in Figure 5.7 (black line), the exposure of the passivated surface to monomer solutions for half an hour did not cause any significant frequency decrease, indicating the low non-specific monomer binding. The elongation rate can be directly obtained either from the slope of the frequency-time-plot or by evaluating the total frequency change over the measurement time. Under the given condition, the value of the frequency change rate $-\frac{d f}{d t}$ was measured to be $2.32 \pm 0.23 \mathrm{~Hz} / \mathrm{min}$. As the surface density of immobilized fibrils was determined to be $734 \pm 89$ molecules $/ \mu \mathrm{m}^{2}$ (as presented in previous Section 5.2.1), the change of the frequency corresponded to an averaged mass increase rate of $159.92 \pm 15.85 \mathrm{fg} / \mathrm{min}$. Normalized by the number of immobilized seed fibrils $N_{\text {Fibril }}$, this yielded an approximate elongation rate of $12.2 \pm 1.2$ monomer molecules per fibril per minute and a rate constant $k=(1.2 \pm 0.1) \times 10^{3} \mathrm{M}^{-1} \mathrm{~s}^{-1}$. This indicates that one monomer attaches and binds to a seed fibril every $4.9 \pm 0.5 \mathrm{~s}$ on average. 
Furthermore, each seed fibril was assumed to consist of two twisted protofibrils detected by EM (4.3.2) and each monomer contributes to a $0.48 \mathrm{~nm}$ length increase along the fibril axis. Therefore, the half-hour fibril growth resulted in a length increase of approximately $88 \mathrm{~nm}$ in total. The length increase was negligible compared to the $3 \mu \mathrm{m}$ by $8 \mu \mathrm{m}$ microchannel dimension, implying that the fibril growth did not affect the fluid flow in the channel. As shown in Figure 5.7, the unchanged slope clearly verified the independence of the growth rate on the fibril length. This independence is important for further quantitative measurements under different conditions, as the rate comparison can be performed with an identical ensemble of fibrils within minutes and the parameters that change the rates can be studied. In addition, the non-specific adsorption of monomer molecules to the functionalized surfaces could be efficiently prevented.

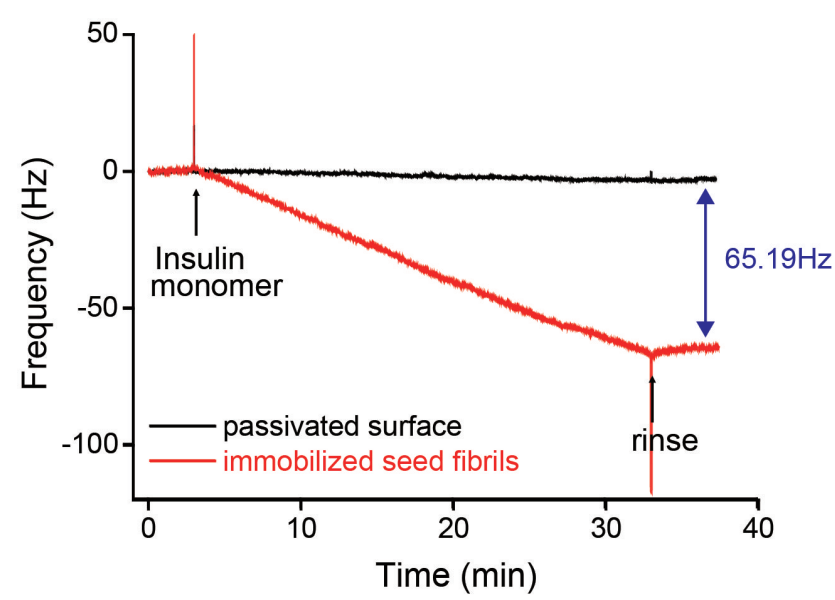

Figure 5.7: Plot showing seed fibril growth with $1 \mathrm{mg} / \mathrm{mL}$ monomer solution for 30 minutes and non-specific binding of monomers on passivated surfaces.

Knowles et al. measured the elongation rate of bovine insulin using QCM detection at room temperature [138]. At the same monomer concentration of $1 \mathrm{mg} / \mathrm{mL}$, the monomer binding time was reported to be $3.1 \pm 1.2 \mathrm{~s}$. The binding time is slightly shorter but within the same order of magnitude with the value obtained by SMR. The slightly faster elongation observed by QCM can be explained by the fact that bovine insulin is more prone to form fibrils than human insulin due to different amino acid residues in three positions [187]. Differences in temperature and buffer could further explain the rate difference.

Figure 5.8 shows that the measurements of the elongation rate constants took less than 5 minutes with three different monomer solutions, consuming ca. 10-30 $\mu \mathrm{L}$ sample volume. However, the equivalent flow-cell volume of the SMR devices was less than $10 \mathrm{pL}-3-6$ orders of magnitude less compared to that of conventional 
biosensors such as QCM and SPR. The low sample consumption was achieved by simultaneously pressurizing the in- and outlet of a bypass channel, slowing down the flow rate in the bypass channel, as presented in Section 3.4.3. Figure 5.8 shows successive measurements of elongation rates on the same fibril ensemble at three different concentrations with (b) and without (a) concentration-induced fluid density change subtracted. The plot (b) shows that the slope or the growth rate increased with the monomer concentration. More studies of the effect of environmental factors on fibril elongation kinetics are described in Section 5.3.1.

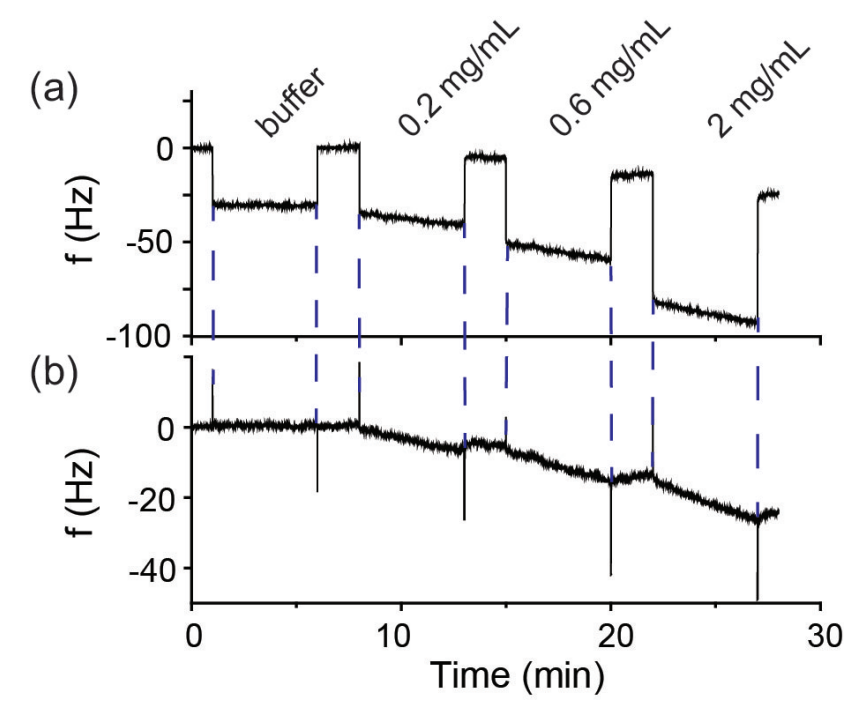

Figure 5.8: SMR measurements of seed fibril elongation with (a) and without (b) fluid density change.

\subsection{Effect of Solution Composition, Temperature and Flow on Insulin Fibril Elongation}

In the search for the fundamental mechanisms and the thermodynamic parameters of the amyloid fibril elongation process, effects of various environmental factors on the elongation rate constants were explored. The tested parameters were focused on the following categories:

- Solution composition. The kinetic dependence on monomer concentration was explored. Moreover, additives such as metal ions, natural products and small aromatic organic compounds were screened. Many of the additives have been suggested to be potential drug candidates to treat amyloid-related diseases as introduced in Section 1.1.3. 
- Temperature. Combined with the measurements with various monomer concentrations, mechanistic models were established and the thermodynamic parameters were measured.

- Flow. The effect of mechanical shear force in the microchannel system was studied.

It is noteworthy that all measurements here were conducted in solutions at $\mathrm{pH}$ 2.0 due to the reduced solubility of insulin monomers at higher $\mathrm{pH}$ values. As shown in Figure 5.9, $0.4 \mathrm{mg}$ insulin monomers were dissolved in $1 \mathrm{~mL}$ buffer solution at $\mathrm{pH}$ in the range of 2.0 to 7.0 . The solutions were filtered with $0.22 \mu \mathrm{m}$ membrane filters and the filtrate concentrations were determined by measuring the absorbance at $280 \mathrm{~nm}$. The plot shows that more than $60 \%$ monomer molecules were filtered out when the $\mathrm{pH}$ was above 4.5 and the monomer solubility decreased with higher $\mathrm{pH}$ values. Therefore, all the monomer solutions in the elongation experiments were set to $\mathrm{pH} 2.0$ in order to lower the risk of data analysis with uncontrolled concentration parameter and the eventually possible clogging problems in the SMR microchannel systems.

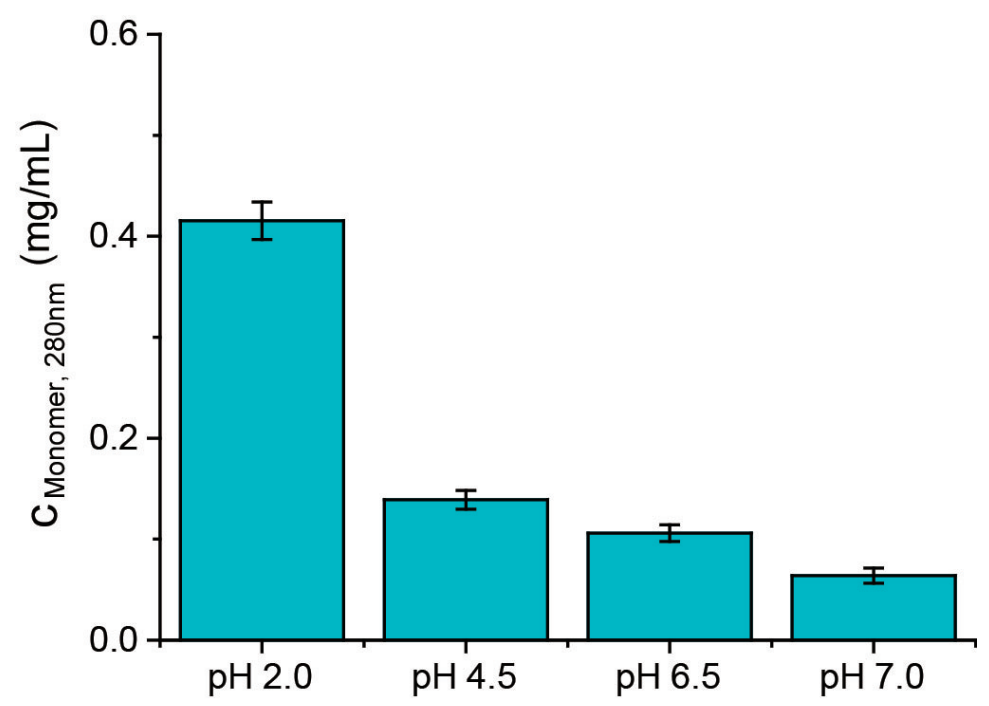

Figure 5.9: Solubility test of insulin monomers at various $\mathrm{pH}$ values detected by UV absorption detection at $280 \mathrm{~nm}$. Insulin was initially dissolved at the same concentration of $0.4 \mathrm{mg} / \mathrm{mL}$ in all buffer solutions at various $\mathrm{pH}$.

\subsubsection{Insulin Elongation Kinetics as a Function of Temperature and Monomer Concentration}

Having established the robustness of the method, the concentration-dependence of the growth rate was examined with monomer concentrations over three orders of 
magnitude $(0.06-6 \mathrm{mg} / \mathrm{mL})$ to probe the elongation process by which monomers were incorporated into growing fibrils. The elongation rates of all the solutions were also measured at different temperatures $\left(20-42^{\circ} \mathrm{C}\right)$, so that the characteristic thermodynamic parameters could be obtained. The measured changes in resonance frequency and the corresponding absolute elongation rates $\frac{d N}{d t}$ were summarized in Figure 5.10. As shown in plot (a), a rate increase with temperature was clearly observable. For example, the results showed a ca. 3.5 times increase in elongation rate as the temperature was changed from $25^{\circ} \mathrm{C}$ to physiological temperature of $37^{\circ} \mathrm{C}$. At a given temperature, the elongation rate increaseed non-linearly with the monomer concentration. The rate increased rapidly at low concentrations until the growth became slower at higher concentrations. This behaviour has also been reported for insulin and other amyloid systems using different detection techniques $[54,138,218]$. It was suggested that not the attachment of precursor proteins but a conformational rearrangement was the rate-limiting step at higher concentration.

A prerequisite for correct on-rates determination by the surface-based SMR measurements is that the elongation detection is not limited by analyte depletion in the microfluidic system $[33,88,240]$. First, the transit time of the monomer solution at the center of the $3 \mu \mathrm{m}$ high and ca. $144 \mu \mathrm{m}$ long microchannel was about $10 \mathrm{~ms}$. The corresponding Peclet number $P e=\frac{v_{c} \frac{H}{2}}{D}$ in the SMR microchannel was two orders of magnitude smaller than in a typical SPR or QCM flow cell (ca. $50 \mu \mathrm{m}$ high and ca. $50 \mathrm{~mm} / \mathrm{s}$ fast flow). This indicates a much faster diffusion from the center of the channel to the wall. Here, $v_{c}$ is the velocity at the center of the channel, $\mathrm{H}$ is the height of the channel and $D$ is the diffusion coefficient that is $1.0 \times 10^{-10} \mathrm{~m}^{2} / \mathrm{s}$ for insulin. Furthermore, it took $4.57 \mathrm{~s}$ to have a monomer molecule bound to a fibril. This long reaction time combined with the short diffusion time $(22.5 \mathrm{~ms})$ resulted in a Damkohler number $D a=0.005$ calculated by $D a=\frac{\text { time(diffusion) }}{\text { time(reaction) }}$. The Damkohler number was much smaller than 1, indicating that the detected binding process was reaction-limited. As such, the transition from fast rate increase at low concentrations to slow rate increase at high concentrations could only be explained with the reaction mechanism.

The concentration-dependent kinetic data were then fitted with a two-step "dock - lock" model, as illustrated in Figure 5.11:

$$
M+F \stackrel{k_{\text {dock }}}{\longrightarrow} F^{*} \stackrel{k_{\text {lock }}}{\longrightarrow} F
$$

Since no dissociation was observed during the measurements, dissociation rate 

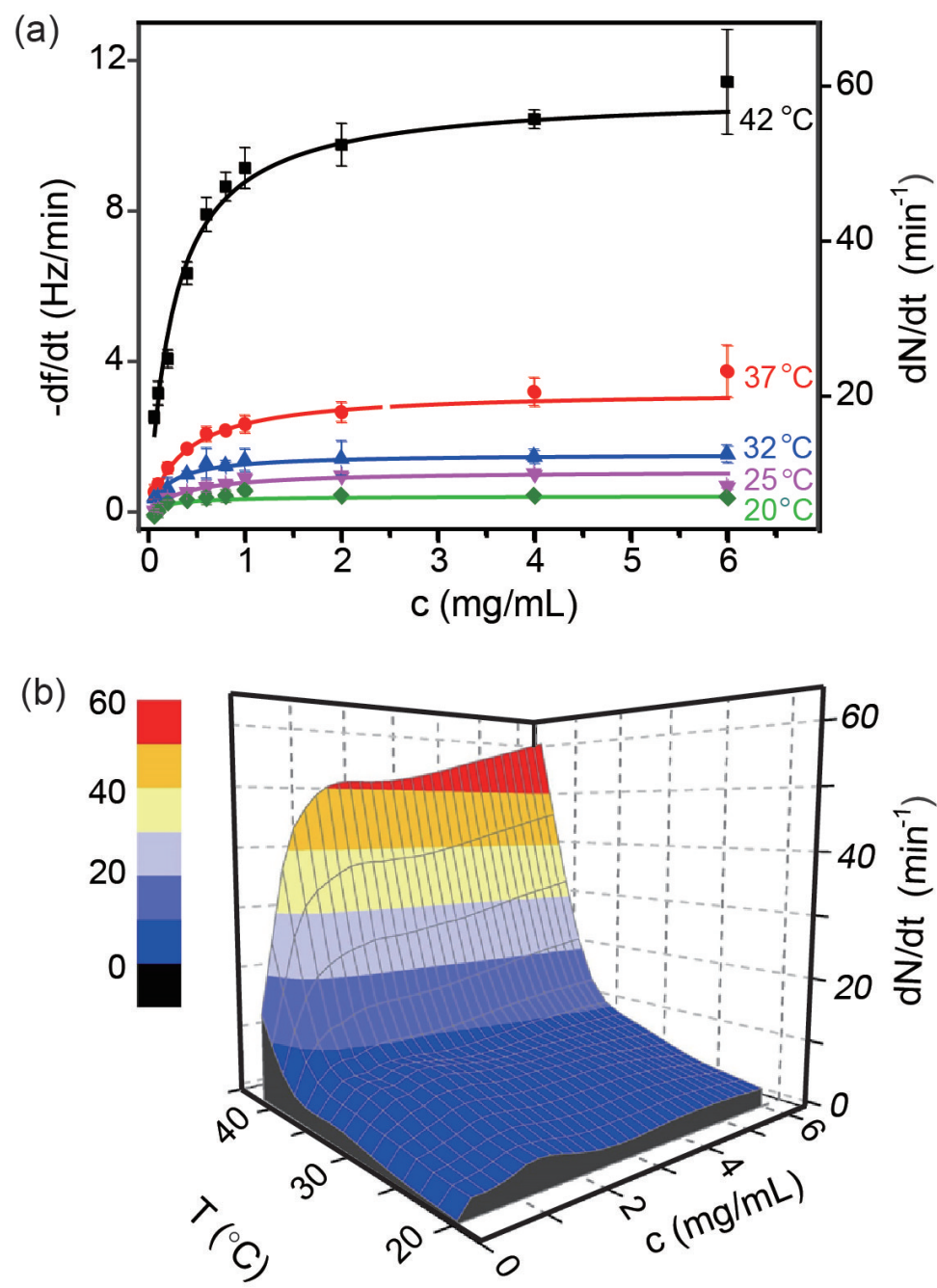

Figure 5.10: Kinetic measurements (a) and 3D-plot (b) of on-rates of seed fibrils at various concentrations and temperature.

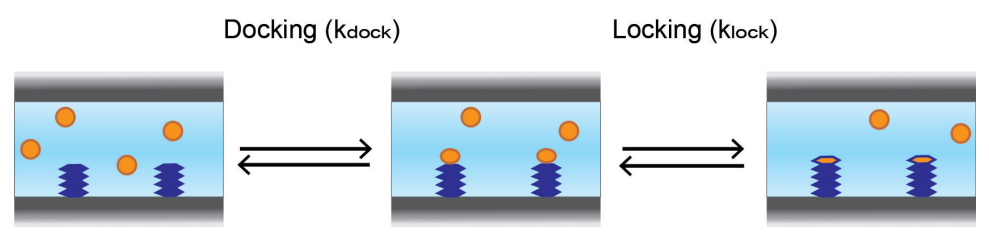

Figure 5.11: Schematic representation of a "dock-lock" model of insulin fibril elongation.

constants are assumed to be negligible for the fitting model. The fitting equation is expressed in Equation 5.2:

$$
y=\frac{d N}{d t}=\frac{k_{\text {lock }} * N_{\text {Fibril }} * c_{\text {Monomer }}}{c_{\text {Monomer }}+\frac{k_{\text {lock }}}{k_{\text {dock }}}}
$$

where $N_{\text {Fibril }}$ is the product of surface density of the fibrils $\sigma_{\text {Fibril }}$ and the surface 
Table 5.1: Fitting parameters for the two-step "dock-lock" elongation mechanism.

\begin{tabular}{ccc}
\hline \hline $\begin{array}{c}\mathrm{T} \\
{\left[{ }^{\circ} \mathrm{C}\right]}\end{array}$ & $\begin{array}{c}k_{\text {dock }} \\
{\left[\times 10^{3} M^{-1} s^{-1}\right]}\end{array}$ & $\begin{array}{c}k_{\text {lock }} \\
{\left[\times 10^{-2} s^{-1}\right]}\end{array}$ \\
\hline 20 & $0.92 \pm 0.46$ & $3.68 \pm 0.56$ \\
25 & $1.40 \pm 0.36$ & $9.49 \pm 1.21$ \\
32 & $3.60 \pm 0.27$ & $13.39 \pm 0.45$ \\
37 & $4.33 \pm 0.38$ & $28.01 \pm 0.56$ \\
42 & $21.12 \pm 0.24$ & $96.63 \pm 3.57$ \\
\hline \hline
\end{tabular}

area S. The surface density of the fibrils $\sigma_{\text {Fibril }}$ was $734 \pm 89$ molecules $/ \mu \mathrm{m}^{2}$ as presented in Section 5.2.1 and the surface area $S$ was $5216 \mu \mathrm{m}^{2}$ with the applied SMR chip. Similar to Equation 5.1 in Section 5.1, the parameters $N$ is the number of the monomer molecules bound to the fibrils, and $c_{\text {Monomer }}$ is the concentration of the monomer solution. In the microchannel, monomers attached to the fibril ends loosely in the dock-step with apparent rate constant $k_{d o c k}$, followed by the lock-step of conformational rearrangement with the rate constant of $k_{\text {lock }}$. As introduced in Section 1.1.2, the "dock-lock"-mechanism has been reported in previous studies using diverse detection techniques, but the number of steps involved in the process are still under debate. In this study, the two-step model can sufficiently describe the data and models of greater complexity with more fitting parameters are not necessary. The fitted rate constants for both dock and lock steps are listed in the Table 5.1.

Furthermore, the rate constants of the dock and lock steps were found to follow Arrhenius law, as expressed by equation 5.3, by plotting logarithms of the rate constants as a function of inverse temperature (Figure 5.12).

$$
k=A * \exp \left(-\frac{E_{A}}{R T}\right)
$$

The data points could be fitted well with a straight line. The activation energy $E_{A}$ was determined from the slope of the fitted line. This activation energy represents the enthalpic activation barrier $\Delta H^{\ddagger}$ in the transition state theory. The values of the activation energy were evaluated to be $27.8 \pm 8.4 \mathrm{kcal} / \mathrm{mol}$ for the dock step and $31.8 \pm 5.2 \mathrm{kcal} / \mathrm{mol}$ for the lock step. Interestingly, the activation energy of the lock step was only slightly higher than the energy of the dock step, indicating that the lock step was the rate-limiting step of the elongation process. Therefore, the 
activation enthalpy of the elongation process was $31.8 \pm 5.2 \mathrm{kcal} / \mathrm{mol}$. Interestingly, the revealed elongation enthalpy is equivalent to the bond energies of 4-15 hydrogen bonds, since enthalpies of hydrogen bonds are in the range of 1.9-6.9 kcal/mol [73]. Following Knowles et al. [138], the Gibbs free energy of activation $\left(\Delta G^{\ddagger}\right)$ can be determined by using equation 5.4 :

$$
\Delta G^{\ddagger}=-R * T * \ln \left(\frac{\frac{d N}{d t}}{\Gamma}\right)
$$

where $\mathrm{R}$ is the gas constant and $\Gamma$ is a kinetic prefactor, which can be calculated using equation 5.5 based on polymer theory on the basis of a simplified model for amyloid fibril growth [31, 138, 146].

$$
\Gamma=10^{3} * c_{\text {Monomer }} * N_{A} * r_{\text {reac }} * F * D
$$

Here, $N_{A}$ is the Avogadro number, $r_{\text {reac }}$ is the encounter volume on the order of $10^{-27} \mathrm{~m}^{3}$, D is the diffusion coefficient of insulin $\left(1.0 \times 10^{-10} \mathrm{~m}^{2} / \mathrm{s}\right)$, and $\mathrm{F}$ is the fibril number per $\mathrm{cm}^{2}$ on the microchannel surface. In the SMR measurements, the Gibbs free energy of activation was calculated to be $6.85 \pm 0.85 \mathrm{kcal} / \mathrm{mol}$ for elongation with $1 \mathrm{mg} / \mathrm{mL}$ monomer solutions at $25^{\circ} \mathrm{C}$. The entropic contribution of the activation $T \Delta S^{\ddagger}$ was determined to be $24.95 \pm 6.05 \mathrm{kcal} / \mathrm{mol}$ using $\Delta G^{\ddagger}=\Delta H^{\ddagger}-T \Delta S^{\ddagger}$. The large entropy of activation may arise from unfolding of proteins and release of bound water molecules.

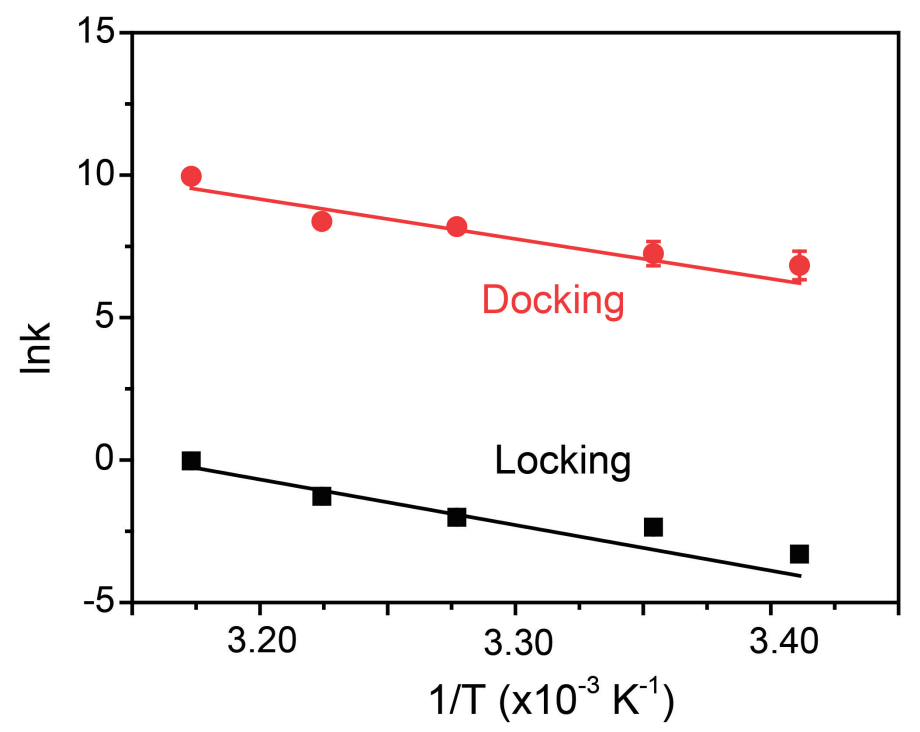

Figure 5.12: Arrhenius plot of logarithms of the rate constants vs. inverse temperature for the dock and lock steps of the elongation processes. 
Table 5.2: Thermodynamic parameters for amyloid-related proteins.

\begin{tabular}{ccccc}
\hline \hline Protein & Approach & $\begin{array}{c}\Delta H^{\ddagger} \text { or } E_{A} \\
{[\mathrm{kcal} / \mathrm{mol}]}\end{array}$ & $\begin{array}{c}T \Delta S^{\ddagger}\left(25^{\circ} \mathrm{C}\right) \\
{[\mathrm{kcal} / \mathrm{mol}]}\end{array}$ & $\begin{array}{c}\Delta G^{\ddagger} \\
{[\mathrm{kcal} / \mathrm{mol}]}\end{array}$ \\
\hline Human insulin & SMR & $31.8 \pm 5.2$ & $25.0 \pm 6.1$ & $6.9 \pm 0.9$ \\
\hline Human insulin [170] & QLS & $25 \pm 2$ & $14.9 \pm 0.6$ & $8 \pm 3$ \\
Bovine insulin [138] & QCM & $24.4 \pm 1.0$ & $16.8 \pm 2.0$ & $6.1 \pm 2$ \\
Human A $\beta[31]$ & QCM & $15.8 \pm 1.9$ & $14.4 \pm 3.2$ & $1.4 \pm 1.0$ \\
$\mathrm{~A} \beta[146]$ & QLS & $23.0 \pm 0.6$ & ca. 16 & ca. 7 \\
\hline \hline
\end{tabular}

The resultant thermodynamic parameters are generally comparable to the literature values reported for different amyloid-related proteins using different approaches, as listed in Table 5.2. Compared to the thermodynamic parameters for human insulin detected by QLS in solution [170], the similar values in SMR measurements suggest that the fibril elongation at the solid-liquid interface is similar to the corresponding process in solution.

\subsubsection{Shear Effect on Insulin Elongation Kinetics}

Vigorous agitation or shaking has been reported to have a dominant effect on the kinetics of amyloid fibril formation over other factors such as concentration and ionic strength [15,188,261]. Moreover, protein solutions are often exposed to shear stresses during bioprocessing steps including centrifugation, fractionation, pumping and filtration [15, 167]. Although much experimental effort has been given to understand the effects of shear flow on amyloid formation in solution, the uncontrolled shear stress under stirring and the heterogeneity of the resulting aggregation products make it challenging to interpret results of conventional experiments to this end $[109,238]$. In microfluidic systems, the small dimensions of the micrometer-sized channels provide laminar flow at very high linear fluid velocities with low Reynolds number and large shear stresses. Microfluidic platforms thus offer unique opportunities to quantitatively study shear stress effects on amyloid elongation kinetics.

In surface-binding-based SMR measurements of this study, the elongation rates of an ensemble of surface fibrils were compared at various shear rates by changing the pressure between the two microchannel ends.

In microfluidic systems, the shear stress $\tau$ of Newtonian fluids in laminar flow is proportional the dynamic viscosity of the fluid $\mu$ and to the velocity gradient in the 
(a)
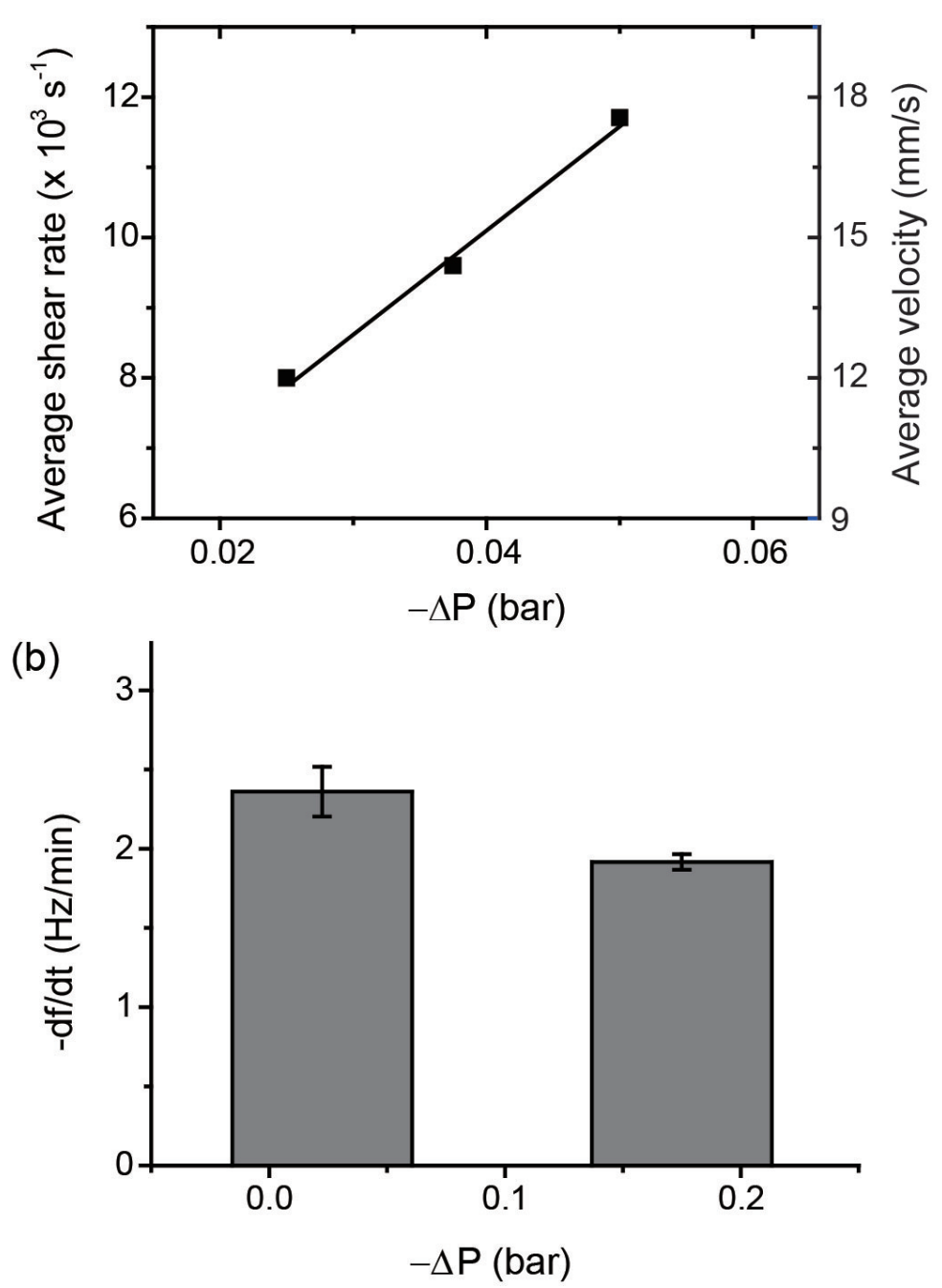

Figure 5.13: Linear correlation of reciprocal of transit time and the pressure drop between two microchannel ends (a) and measured frequency change rate for two different pressure drops using SMR (b).

direction perpendicular to the plane of shear, as expressed in equation 5.6.

$$
\tau=\mu \frac{\partial u}{\partial y}
$$

Here, $y$ denotes the distance perpendicular to the boundary surface and $u$ denotes the flow velocity. In rectangular microchannels, the average shear stress $\bar{\tau}$ over the perimeter of the channel and the Hagen-Poiseuille flow can be simply described using the equations 5.7 and 5.8 with channel dimensions (height $h$, width $w$ and length $l$ ), pressure drop $\Delta P$ and the average volumetric flow rate $Q[110,165]$.

$$
\bar{\tau}=-\frac{12 Q \mu}{h^{2} w}
$$




$$
Q=-\frac{h^{3} w \Delta P}{12 \mu l}
$$

Therefore, a linear correlation between the shear stress and the pressure drop is expected, as expressed in equation 5.9.

$$
\bar{\tau}=\frac{h}{l} * \Delta P
$$

The fluid flow inside the SMR microchannels was driven by applying pressure to the inlets and outlets. In the $3 \mu \mathrm{m}$ high and $8 \mu \mathrm{m}$ wide resonator micorchannel, the shear stress is thus theoretically in the range of $(0.94-9.4) \times 10^{-3}$ bar when applying a pressure drop of $0.025-0.25$ bar. In conventional microfluidic channels, the typical channel height is in the range of $5-100 \mu \mathrm{m}$ and the width is $10-100 \mu \mathrm{m}$. By applying 0.001-0.01 bar pressure drop [82,165], the shear rates are normally one or two orders of magnitude less than the shear rates in the SMR microchannels.

However, the shear stress or the flow velocity was difficult to predict accurately, as the actual pressure drop could deviate from the theoretical value. Clogging, partial clogging or minor obstruction of the channel could not be easily recognized from the data. To this end, polystyrene beads were added to the $1 \mathrm{mg} / \mathrm{mL}$ monomer solutions to trace the flow velocity by measuring the transit time. In the ca. $144 \mu \mathrm{m}$ long resonator channel, the average flow velocity $u_{\text {average }}$ of the parabolic velocity profiles was then determined using $u_{\text {average }}=\frac{L}{t_{\text {transit }}}$ with the transit time $t_{\text {transit }}$. The average flow velocity is linearly proportional to the applied pressure drop.

The plot (a) of Figure 5.13 verifies that the flow velocity in the experiment correlated linearly with the pressure drop as expected. However, the evaluation was only done with pressure drop in the range of $0.025-0.25$ bar. At higher pressure drops, the transit time could not be resolved, since the beads passed too fast through the resonator microchannel. However, the flow velocity could be roughly estimated using the established calibration plotted in Figure 5.13 (a).

Furthermore, the elongation rates were compared with different pressure drops. As shown in the plot (b) of Figure 5.13, the elongation rate with 0.02 bar pressure drop was comparable to the rate with 0.18 bar pressure drop, indicating that a 9 times increase in pressure drop did not result in a significant change in elongation rate. Previously, Hill et al. explored the shear stress effect on $\beta$-lactoglobulin fibril elongation using a Couette cell combined with ThT-fluorescence detection [109]. They observed a rate increase with shear rate ranging from 50 to $200 \mathrm{~s}^{-1}$. The shear rates in the SMR measurements were $(2-20) \times 10^{4} \mathrm{~s}^{-1}$ with transit time of 
ca. 1-10 ms, which were 2-3 orders of magnitude higher than the shear rates in the Couette cell experiments. It was suggested in some previous studies that a shear rate of ca. $10^{7} \mathrm{~s}^{-1}$ would be required to denature a small globular protein [15]. The shear rates in SMR measurements were below this range. However, it is likely that the shear stress effects on the elongation process have saturated in the SMR detection range. Although with current microchannel dimensions and the pressure regulation system the shear stress dependence of the amyloid elongation is below detection limit, microfluidic systems with adequately designed channel dimensions and pressure regulation in a lower range could be potential platforms for such measurements.

\subsubsection{Influence of Additives on Elongation Kinetics}

Specific chemical compounds can significantly affect the amyloid elongation process. Screening techniques to search for inhibitors to prevent fibril growth are therefore of importance for clinical applications. However, among the large variety of the possible inhibitors which are summarized in Section 1.1.3, the inhibition effects of many candidates remain under debate, since opposite conclusions have been drawn by using different detection techniques [192,279]. Here, selected candidates from three categories (metal ions, natural products and small aromatic organic compounds) were screened using SMR measurements. Additives were dissolved in the $0.4 \mathrm{mg} / \mathrm{mL}$ monomer solutions at concentrations spanning several orders of magnitude including the reported effective values. The elongation rates were measured at $37^{\circ} \mathrm{C}$ while successively introducing the monomer solutions with an increasing concentration of the additives. In addition, two reference measurements with the same monomer concentrations but without additives were carried out before and after the additive measurements. In this way, more insight into inhibition mechanisms can be given by knowing whether the inhibitors interact predominately with monomers or with fibrils.

\subsubsection{Kinetics with Additional Salt and Metal Ions}

Four metal ion salts $\left(\mathrm{NaCl}, \mathrm{ZnCl}_{2}, \mathrm{MgCl}_{2}, \mathrm{CaCl}_{2}\right)$ were selected here to explore effect on elongation kinetics. So far, their effect on the elongation kinetics have not been completely revealed and understood.

Sodium chloride $\mathrm{NaCl}$ is frequently used as a component in buffer solutions. Nielsen et al. studied the effect of ionic strength on insulin fibrillation kinetics using the ThT-fluorescence assay and showed a slight decrease in lag time and in the 
apparent rate constants with increased $\mathrm{NaCl}$ concentration up to $50 \mathrm{mM}$ [188].

$\mathrm{Zn}$ (II) ions have been reported to be able to inhibit insulin fibrillation at physiological $\mathrm{pH}$ values by forming stable and soluble $\mathrm{Zn}(\mathrm{II})$-insulin hexamer complexes. The inhibitory effect has been suggested to be very strong at $3.5 \mu \mathrm{M}$ at pH 7.3 and become weaker at pH 5.5 [191]. Tougu et al. demonstrated that $\mathrm{Zn}$ (II) inhibited the $\mathrm{A} \beta_{42}$ aggregation with $\mathrm{IC}_{50}$ value of $1.8 \mu \mathrm{M}$ [249].

Additionally, $\mathrm{MgCl}_{2}$ and $\mathrm{CaCl}_{2}$ were tested due to their physiological relevance. $\mathrm{Mg}$ (II) and $\mathrm{Ca}(\mathrm{II})$ are essential elements in the human body. While $\mathrm{Ca}(\mathrm{II})$ plays an important role in signal transduction pathways, neurotransmitter release etc., $\mathrm{Mg}$ (II) is a relevant cofactor of numerous enzymes and is involved in the treatment of many diseases including diabetes mellitus [100,164].

Figure 5.14 shows a summary of growth rates for different concentrations of all four additives. The rates were normalized by the initial reference rates. In general, no significant inhibition was observed with all four additives, indicating that all the metal ions did not stabilize the monomer and fibril structures and suppress the elongation process at the concentrations that were tested. Conversely, the additives accelerated the elongation rates at some of the concentrations. The concentration dependence of the growth rates of all the additives was similar. The rates increased initially, reached the maximum at concentrations in the range of $60-100 \mathrm{mM}$ and decreased at higher concentrations. $\mathrm{MgCl}_{2}$ and $\mathrm{CaCl}_{2}$ showed a larger acceleration effect than $\mathrm{ZnCl}_{2}$ and $\mathrm{NaCl}$. However, mechanisms underlying the acceleration effect of these additives have not been fully understood.

\subsubsection{Kinetics with Additional Natural Products}

Some microorganisms, e.g. hyperthermophiles, accumulate small stress molecules as a means of surviving at temperatures higher than $100^{\circ} \mathrm{C}$ [9]. This indicates that small stress molecules may be able to stabilize the native conformation of proteins. Arora et al. have reported that the small stress molecules ectoine, trehalose, citrulline and betaine as natural products strongly suppressed insulin amyloid formation at concentrations as high as $300 \mathrm{mM}$ [9]. In particular, ectoine was suggested to inhibit the amyloid elongation. However, the results of SMR kinetic measurements with ectoine and trehalose (30-300 $\mathrm{mM})$ showed that both additives did not change the elongation rates significantly. 


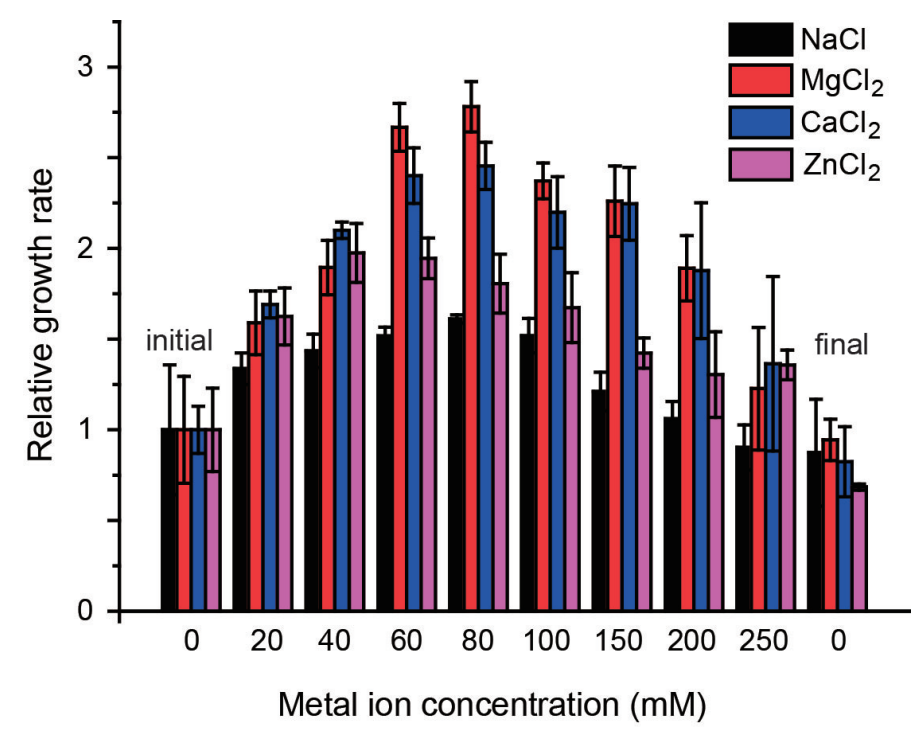

Figure 5.14: Test of elongation rates with additives of metal ions ( $\mathrm{NaCl}, \mathrm{ZnCl}_{2}, \mathrm{MgCl}_{2}$, $\mathrm{CaCl}_{2}$ ). The screening procedure started with the initial injection of the $0.4 \mathrm{mg} / \mathrm{ml}$ monomer solutions, followed by the injection of the monomer solutions with increased additive concentrations and the final injection of the monomer solution.

\subsubsection{Kinetics with Small Aromatic Organic Compounds}

Small aromatic compounds have been reported as inhibitors of fibril formation $[142,158]$. It has been suggested that the $\pi$-ring structure of the compounds could interact with amyloids by stacking with the side chains or by hydrophobic interaction. This could stabilize the fibrils or monomers and give rise to the antiaggregation potency by preventing the formation of complex $\beta$-sheet fibril structures.

Inhibition tests were carried out with two aromatic compounds: pthalocyanine tetrasulfonate (PcTS) and methylene blue.

PcTS has been recently reported to efficiently reduce the pathogenic amyloid deposits of tau protein using a combination of NMR and SAXS [5, 158]. The inhibition experiments by Akoury et al. were conducted with PcTS concentrations ranging from $200 \mathrm{pM}$ to $200 \mu \mathrm{M}$, and the $I C_{50}$ value was determined to be $1.25 \mu \mathrm{M}$. In the SMR measurements, PcTS was added to the monomer solution at concentrations ranging from 1 to $100 \mu \mathrm{M}$. As shown in Figure 5.16(a), a significant change in elongation rates was only observed at high concentrations of $100 \mu \mathrm{M}$. However, precipitation was also observed at this high concentration. At low concentrations that are at or below the $I C_{50}$, the rates were comparable to the reference solutions without PcTS addition. The unchanged elongation rates of $0.4 \mathrm{mg} / \mathrm{mL}$ monomer solutions before and after the injection of additive solutions (a, inset) indicate that the aromatic PcTS compound did not interact with the surface fibrils. 

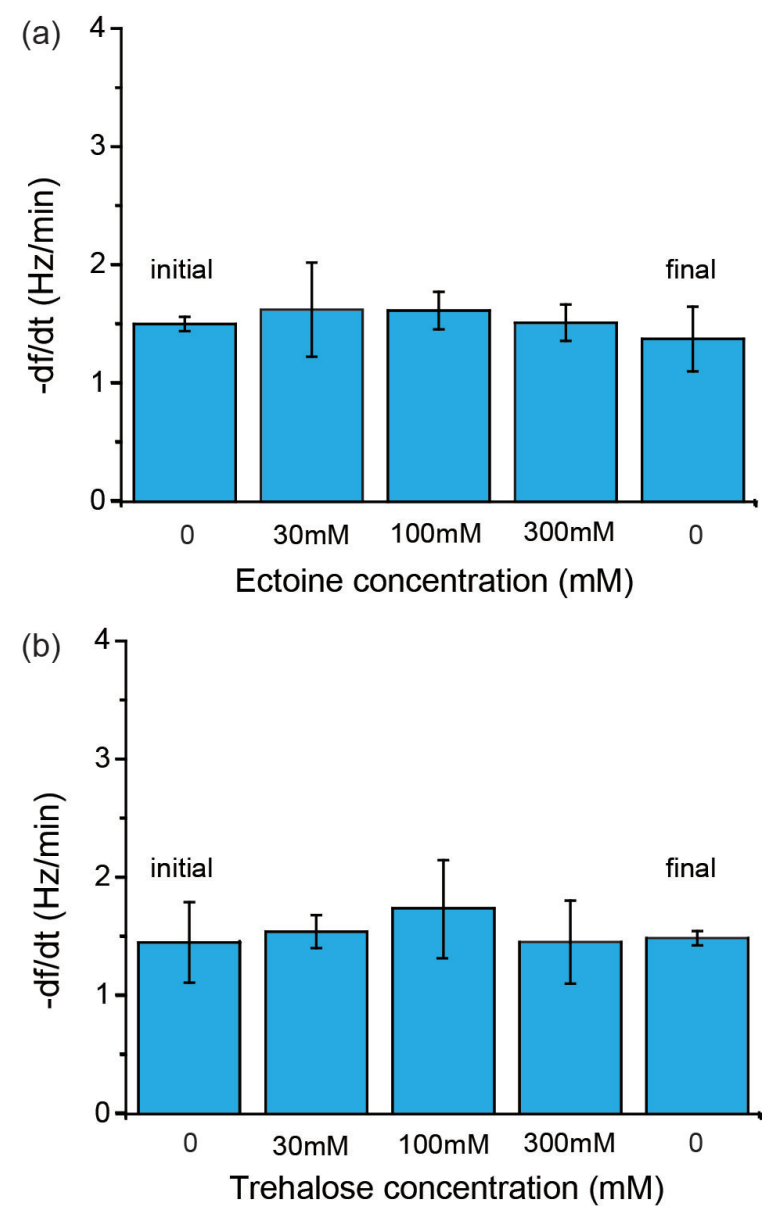

Figure 5.15: Test of elongation rates with additives of ectoine (a) and trehalose (b). The screening procedure started with the initial injection of the $0.4 \mathrm{mg} / \mathrm{ml}$ monomer solutions, followed by the injection of the monomer solutions with increased additive concentrations and the final injection of the monomer solution.

Furthermore, the application of methylene blue has been suggested to be able to attenuate amyloid plaques and slow down the progression of Alzheimer's disease [198]. Oz et al. showed in their experiments that the compound could inhibit $\mathrm{A} \beta_{42}$ aggregation with an $I C_{50}$ value of $2.3 \mu \mathrm{M}$. Here elongation rates were measured with addition of methylene blue to the monomer solutions at concentrations of 1.25-125 $\mu \mathrm{M}$. Figure 5.16 (b) shows that methylene blue had an inhibitory effect on the elongation process. Under the experimental conditions, maximum impact was found at low concentrations. Further tests need to be conducted to explore the inhibition at low concentrations in more detail. 

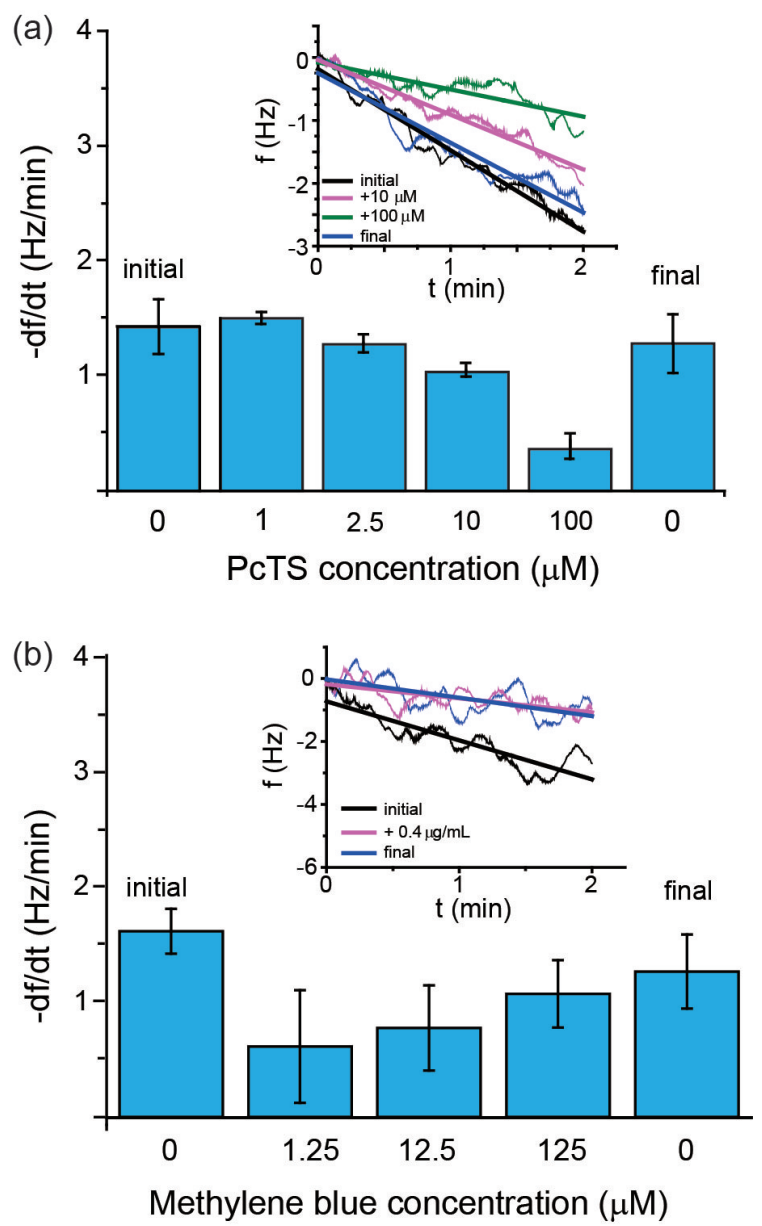

Figure 5.16: Test of elongation rates with additives of PcTS (a) and methylene blue (b). The screening procedure started with the initial injection of the $0.4 \mathrm{mg} / \mathrm{mL}$ monomer solutions, followed by the injection of the monomer solutions with increasing additive concentrations and the final injection of the monomer solution. Insets show the frequency monitoring of monomer solutions before (initial) and after (final) the injection of the solutions with the two additives.

\subsection{Dissociation Detection of Immobilized Insulin Fibrils}

In search of conditions and compounds that could break the stable fibrils into monomers or short fragments, dissociation experiments were performed by monitoring the rates of frequency change under various conditions. The tested parameters were temperature, $\mathrm{pH}$, and the same compounds that were used for additive tests in Section 5.3.3.

The insulin fibrils on the channel surface were exposed to running buffers at pH 2 and pH 7, respectively. Since fibrils were generally very stable on the surface 
at temperature higher than $37^{\circ} \mathrm{C}$ (data not shown), the dissociation was detected at temperatures ranging from $10-37^{\circ} \mathrm{C}$. After the temperature was changed, the frequency measurement started after a new equilibrium was reached, which took approximately 20 minutes. To exclude systematic errors due to the direction of temperature change, the rates were measured after cooling down and heating up the devices respectively. The temperature-dependence of fibril dissociation, as shown in Figure 5.17, indicates that the dissociation rates were in general small (within $0.6 \mathrm{~Hz} / \mathrm{min})$. The rates were not much different from the background signal, indicating that fibrils are relatively stable. However, it was also observable that the fibrils dissociated slightly faster with decreased temperature. This observation is in agreement with previous studies, which suggested that amyloid fibrils are highly sensitive to low temperature [135]. There was no significant difference in dissociation rates between $\mathrm{pH} 2$ and $\mathrm{pH} 7$, showing that $\mathrm{pH}$ values had a minor effect on fibril stability.

Furthermore, the dissociation measurements were performed by loading the buffer solutions with additives of metal ions ( $\mathrm{NaCl}, \mathrm{ZnCl}_{2}, \mathrm{MgCl}_{2}, \mathrm{CaCl}_{2}$ ), natural products (ectoine, trehalose) and small organic molecules (PcTS, methylene blue) at $\mathrm{pH} 2$ and $37^{\circ}$ Cinto the microchannel with fibrils on the surface. The reference signal was obtained by measuring the frequency shift when buffer without additives was in the channel. All measured dissociation rates were comparable to the reference value of ca. $-0.158 \pm 0.07 \mathrm{~Hz} / \mathrm{min}$, implying that the tested compounds were not able to degrade stable fibrils.

In conclusion, although ideal candidates to dissociate fibrils have not been found in the dissociation experiment, the surface-binding based SMR method is demonstrated to be a reliable platform for the screening of potential drugs to dissolve fibrils. 

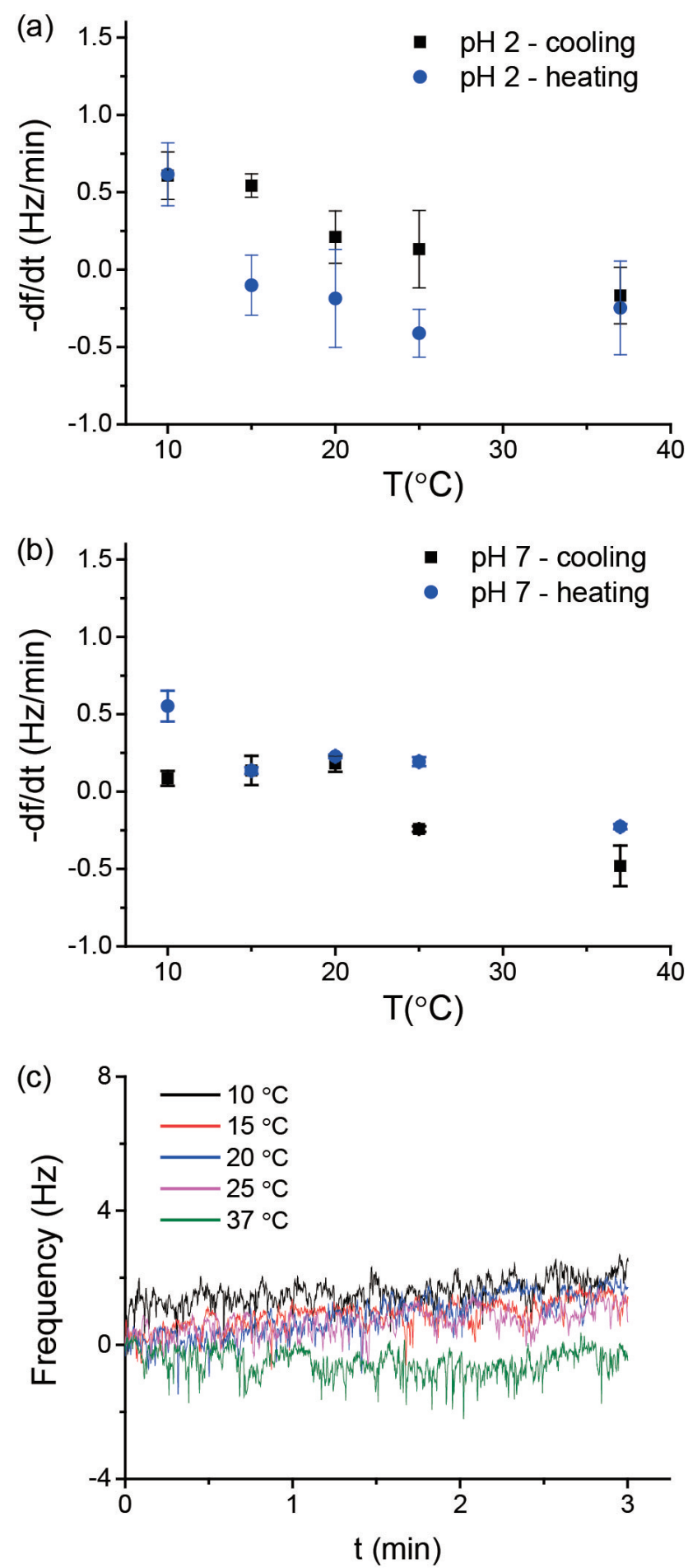

Figure 5.17: Dissociation monitoring of insulin fibrils at $\mathrm{pH} 2$ (a) and $\mathrm{pH} 7$ (b). Time courses of the dissociation monitoring at various temperatures with buffer solutions at $\mathrm{pH} 7$ (c). 


\section{Chapter 6}

\section{Conclusions}

This thesis demonstrates a novel microfluidic approach using SMR devices to quantitatively study the kinetics of amyloid growth by mass. Amyloid formation consists of many elementary steps including the formation of seeds by nucleation and subsequent elongation to mature insoluble fibrils. In the surface-based SMR measurements, short amyloid seed fibrils are immobilized on the microchannel surfaces, and the elongation rates as well as the rate constants are determined by monitoring the mass change of the fibril ensemble over time. Using insulin as a model, comprehensive experimental data sets of measured fibril elongation kinetics shed light on the thermodynamic parameters and the underlying mechanisms that govern this process. Compared to conventional dye-binding assays in bulk solutions, such as ThT-fluorescence, the label-free method is sensitive to all states of the aggregation, avoids the risk of interfering with the reaction, and enables accurate rate constant determination independent of the stoichiometry of the dye binding. Compared to current biosensor techniques, the microfluidic trasducer system achieves further miniaturization of the detection devices with higher mass resolution.

Reliable immobilization of seed fibrils on the surface of closed microchannels is the basis for accurate kinetic measurements. Since the surfaces of SMR devices employed in this study are not transparent, reliable immobilization techniques have been developed in transparent microchannel systems and are later transferred to the SMR. Electrostatic interaction using PLL and covalent coupling using aminosilane and glutaraldehyde have been compared and the bio-functionality of the seed fibrils has also been tested. Stability and kinetic reproducibility tests have revealed that only covalently immobilized fibrils are sufficiently stable inside the microchannel for quantitative elongation rate measurements. Furthermore, covalent coupling is superior to the electrostatic PLL-linking due to lower non-specific adsorption 
of proteins after passivating the reactive functional groups. Therefore, covalent coupling has been applied for all kinetic measurements in this thesis.

The robust covalent protein coupling on the microchannel surface consists of multiple steps, each of which is quantitatively validated:

First, vapor phase deposition of aminosilanes has been successfully developed to functionalize the surfaces of closed microchannel systems with reactive amine groups. The method overcomes numerous challenges associated with the microfluidic environment such as cross-contamination of reagents by dispersion and clogging. The generated amine functional layers are uniform, stable and can be regenerated reproducibly. The applied deposition method furthermore allows batch fabrication of microchannels with functional groups. All these features have been assessed using a novel method that allows quantitative fluorescence-based imaging of surface functional groups inside assembled micro- and nanochannels.

Difficulties in fluorescence quantification of surface functional groups have been recognized by observing the opposing effect of photobleaching and self-quenching due to the dense packing of fluorophors. The use of competitive labeling with stable fluorescent and non-fluorescent molecules eliminates the complexity of selfquenching and results in excellent linearity between the area density of functional groups and fluorescence intensity. Reactive amine groups with a surface density of $55 \pm 9 \mathrm{pmol} / \mathrm{mm}^{2}$ are reproducibly generated by vapor deposition using ATPMS for $16 \mathrm{~h}$ at $80^{\circ} \mathrm{C}$.

Subsequently, glutaraldehyde has been used to further functionalize surface amine groups with aldehyde groups, which couple to amine groups of proteins by Schiff's base formation, followed by the use of a reduction agent to stabilize the labile bonds. To characterize protein binding capacity, GFP moleucles are immobilized on the surface, revealing a packing density of $1.2 \pm 0.5 \mathrm{pmol} / \mathrm{mm}^{2}$.

Uniform seed fibrils of $97.6 \pm 20.1 \mathrm{~nm}$ as validated by EM are prepared and immobilized in the SMR. Based on the size of seeds measured by EM and the total mass of the immobilized layer, the surface density of immobilized seed fibrils has been determined to be $734 \pm 89$ molecules $/ \mu \mathrm{m}^{2}$ with fibril-fibril distance of $37 \pm 13 \mathrm{~nm}$. This has also been validated using AFM.

Upon completion of the development and characterization of the detection platform, the kinetics of insulin seed fibril formation have been systematically explored. In agreement with the theory that amyloid elongation is a bimolecular reaction, the SMR measurements show a continuous linear fibril growth at a rate 
that a monomer on average binds to a fibril every $4.9 \pm 0.5 \mathrm{~s}$, corresponding to the rate constant of $(1.2 \pm 0.1) \times 10^{3} \mathrm{M}^{-1} \mathrm{~s}^{-1}$, in a $1 \mathrm{mg} / \mathrm{mL}$ monomer solution at $37^{\circ} \mathrm{C}$. This result is comparable with reported literature values detected by QCM. To systematically study the impact of monomer concentration and temperature on the elongation kinetics of amyloid fibrils, a large number of elongation rates have been measured under various conditions. A temperature reduction from $37^{\circ} \mathrm{C}$ to $25^{\circ} \mathrm{C}$ results in a 3.5 times decrease in elongation rate, and the concentrationdependence of the elongation rates is non-linear, showing a rapid increase at low monomer concentrations and a slow increase at high concentrations. This non-linear behaviour has been modeled with a two-step "dock-lock"-mechanism, by which a conformational change of the lock-step is the rate-limiting step of the process. Thermodynamic parameters have also been obtained from the data, showing that fibril elongation is in general enhalpically unfavorable but entropically favorable. The detected enthalpy of activation from the SMR measurements, $31.8 \pm 5.2 \mathrm{kcal} / \mathrm{mol}$, is comparable with the reported values for insulin and other amyloid proteins measured by QCM and QLS, suggesting that this order of magnitude could be characteristic for the activation energy of amyloid elongation processes in general.

Finally, the surface-based SMR detection has been applied to explore the effects of environmental factors such as shear stress, metal ions, natural products and small organic compounds. Shear rate, generated by applying a pressure drop of 0.025-0.25 bar in this study, has been proved to have no measurable impact on the elongation rate, likely because the shear stress effects on the elongation process have saturated with high shear rates in SMR detection. By comparing the elongation rates with the reference monomer solution without additives on the identical fibril ensemble, it has been found that metal ion additives ( $\mathrm{NaCl}, \mathrm{MgCl}_{2}, \mathrm{CaCl}_{2}$ and $\mathrm{ZnCl}_{2}$ ) generally accelerate the elongation at concentrations in the range of $60-100 \mathrm{mM}$. Natural products (ectoine and trehalose) do not change the rates. PcTS inhibits the elongation at high concentration of $200 \mu \mathrm{M}$ while methylene blue slows down the elongations at low concentrations.

The mechanisms behind the effects of additives on the elongation kinetics have not been fully studied in this thesis and need to be addressed in further work. However, this approach has shown its capability and potential of searching for therapeutic drugs for amyloid-related diseases.

In addition, dissociation of the immobilized fibrils off the microchannel surface has been monitored under various conditions $(\mathrm{pH}$, temperature and addition of the compounds applied in the association tests). It has been found that $\mathrm{pH}$ and 
the selected additives do not efficiently degrade the stable fibrils. It has been observed that lowering temperature slightly increases the fibril dissociation rates, although these rates are not much different from the background signal. In general, the dissociation monitoring is suggested to be a promising in situ approach for screening of potential fibril degradation candidates.

Another important future goal is to study early aggregation events by surfacebased SMR measurements. In the current study, kinetic measurements with immobilized monomers instead of seed fibrils on the microchannel surface do not show significant signals of early nucleation processes, possibly due to the detection limit of the system or low binding capacity using the current immobilization techniques. This may be improved by a new generation of SMR devices with smaller microchannel dimensions and higher sensitivity. In addition, much effort will be given to optimize the immobilization techniques so that binding capacity of monomers can be maximized. 


\section{Bibliography}

[1] J. Adamcik and R. Mezzenga. Study of amyloid fibrils via atomic force microscopy. Current Opinion in Colloid $\mathscr{6}$ Interface Science, 17(6):369-376, 2012.

[2] A. Aguzzi and T. O'Connor. Protein aggregation diseases: pathogenicity and therapeutic perspectives. Nature Reviews Drug Discovery, 9(3):237-248, 2010.

[3] K. Akasaka, A. R. A. Latif, A. Nakamura, K. Matsuo, H. Tachibana, and K. Gekko. Amyloid protofibril is highly voluminous and compressible. Biochemistry, 46(37):10444-10450, 2007.

[4] C. Akkermans, A. J. Van der Goot, P. Venema, E. Van der Linden, and R. M. Boom. Properties of protein fibrils in whey protein isolate solutions: Microstructure, flow behaviour and gelation. International Dairy Journal, 18(10-11):1034-1042, 2008.

[5] E. Akoury, M. Gajda, M. Pickhard, J. Biernat, P. Soraya, C. Griesinger, E. Mandelkow, and M. Zweckstetter. Inhibition of tau filament formation by conformational modulation. Journal of the American Chemical Society, 135(7):2853-2862, 2013.

[6] D. Ami, A. Natalello, M. Lotti, and S. M. Doglia. Why and how protein aggregation has to be studied in vivo. Microbial Cell Factories, 12, 2013.

[7] J. L. Arlett and M. L. Roukes. Ultimate and practical limits of fluid-based mass detection with suspended microchannel resonators. Journal of Applied Physics, 108(8), 2010.

[8] C. H. Arnaud. Tools for amyloids. Chemical \& Engineering News, 87(24):1013, 2009.

[9] A. Arora, C. Ha, and C. B. Park. Inhibition of insulin amyloid formation by small stress molecules. Febs Letters, 564(1-2):121-125, 2004.

[10] Y. Asanomi, H. Yamaguchi, M. Miyazaki, and H. Maeda. Enzyme-Immobilized Microfluidic Process Reactors. Molecules, 16(7):6041-6059, 2011. 
[11] T Ban, D Hamada, K Hasegawa, H Naiki, and Y Goto. Direct observation of amyloid fibril growth monitored by thioflavin $\mathrm{T}$ fluorescence. Journal of Biological Chemistry, 278(19):16462-16465, 2003.

[12] T. Ban, M. Hoshino, S. Takahashi, D. Hamada, K. Hasegawa, H. Naiki, and Y. Goto. Direct observation of a beta amyloid fibril growth and inhibition. Journal of Molecular Biology, 344(3):757-767, 2004.

[13] A. Bange, H. B. Halsall, and W. R. Heineman. Microfluidic immunosensor systems. Biosensors \& Bioelectronics, 20(12):2488-2503, 2005.

[14] A. I. Bartlett and S. E. Radford. An expanding arsenal of experimental methods yields an explosion of insights into protein folding mechanisms. Nature Structural \&f Molecular Biology, 16(6):582-588, 2009.

[15] I. B. Bekard, P. Asimakis, J. Bertolini, and D. E. Dunstan. The effects of shear flow on protein structure and function. Biopolymers, 95(11):733-745, 2011.

[16] L. M. Bellan, D. Wu, and R. S. Langer. Current trends in nanobiosensor technology. Wiley Interdisciplinary Reviews-Nanomedicine and Nanobiotechnology, 3(3):229-246, 2011.

[17] O. G. Berg and P. H. Vonhippel. Diffusion-controlled macromolecular interactions. Annual Review of Biophysics and Biophysical Chemistry, 14:131-160, 1985 .

[18] T. Bernas, M. Zarebski, P. R. Cook, and J. W. Dobrucki. Minimizing photobleaching during confocal microscopy of fluorescent probes bound to chromatin: role of anoxia and photon flux (vol 215, pg 281, 2004). Journal of Microscopy-Oxford, 216:197-197, 2004.

[19] S. K. Bhatia, L. C. Shriverlake, K. J. Prior, J. H. Georger, J. M. Calvert, R. Bredehorst, and F. S. Ligler. Use of thiol-terminal silanes and heterobifunctional crosslinkers for immobilization of antibodies on silica surfaces. $A n$ alytical Biochemistry, 178(2):408-413, 1989.

[20] M. Biancalana and S. Koide. Molecular mechanism of thioflavin-t binding to amyloid fibrils. Biochimica Et Biophysica Acta-Proteins and Proteomics, 1804(7):1405-1412, 2010.

[21] H. K. L. Blackley, G. H. W. Sanders, M. C. Davies, C. J. Roberts, S. J. B. Tendler, and M. J. Wilkinson. In-situ atomic force microscopy study of betaamyloid fibrillization. Journal of Molecular Biology, 298(5):833-840, 2000. 
[22] N. Blow. Microfluidics: in search of a killer application. Nature Methods, 4(8):665-668, 2007.

[23] D. R. Booth, M. Sunde, V. Bellotti, C. V. Robinson, W. L. Hutchinson, P. E. Fraser, P. N. Hawkins, C. M. Dobson, S. E. Radford, C. C. F. Blake, and M. B. Pepys. Instability, unfolding and aggregation of human lysozyme variants underlying amyloid fibrillogenesis. Nature, 385(6619):787-793, 1997.

[24] E. M. Bradbury, L. Brown, A. R. Downie, A. Elliott, R. D. B. Fraser, W. E. Hanby, and T. R. R. Mcdonald. Cross-beta structure in polypeptides of low molecular weight. Journal of Molecular Biology, 2(5):276-\&, 1960.

[25] J. Brange, L. Andersen, E. D. Laursen, G. Meyn, and E. Rasmussen. Toward understanding insulin fibrillation. Journal of Pharmaceutical Sciences, 86(5):517-525, 1997.

[26] T Braun, V Barwich, MK Ghatkesar, AH Bredekamp, C Gerber, M Hegner, and HP Lang. Micromechanical mass sensors for biomolecular detection in a physiological environment. Physical Review E, 72(3, 1), SEP 2005.

[27] A. C. Brorsson, J. R. Kumita, I. MacLeod, B. Bolognesi, E. Speretta, L. M. Luheshi, T. P. J. Knowles, D. A. Lomas, C. M. Dobson, and D. C. Crowther. Methods and models in neurodegenerative and systemic protein aggregation diseases. Frontiers in Bioscience-Landmark, 15:373-396, 2010.

[28] H. Brunner, T. Vallant, U. Mayer, H. Hoffmann, B. Basnar, M. Vallant, and G. Friedbacher. Substrate effects on the formation of alkylsiloxane monolayers. Langmuir, 15(6):1899-1901, 1999.

[29] A. K. Bryan, A. Goranov, A. Amon, and S. R. Manalis. Measurement of mass, density, and volume during the cell cycle of yeast. Proceedings of the National Academy of Sciences of the United States of America, 107(3):999-1004, 2010.

[30] A. K. Buell, A. Dhulesia, M. F. Mossuto, N. Cremades, J. R. Kumita, M. Dumoulin, M. E. Welland, T. P. J. Knowles, X. Salvatella, and C. M. Dobson. Population of nonnative states of lysozyme variants drives amyloid fibril formation. Journal of the American Chemical Society, 133(20):7737-7743, 2011.

[31] A. K. Buell, A. Dhulesia, D. A. White, T. P. J. Knowles, C. M. Dobson, and M. E. Welland. Detailed analysis of the energy barriers for amyloid fibril growth. Angewandte Chemie-International Edition, 51(21):5247-5251, 2012. 
[32] A. K. Buell, C. M. Dobson, T. P. J. Knowles, and M. E. Welland. Interactions between amyloidophilic dyes and their relevance to studies of amyloid inhibitors. Biophysical Journal, 99(10):3492-3497, 2010.

[33] T. P. Burg. Suspended Microchannel Resonators for Biomolecular Detection. PhD thesis, Massachusetts Institute of Technology, 2005.

[34] T. P. Burg, M. Godin, S. M. Knudsen, W. Shen, G. Carlson, J. S. Foster, K. Babcock, and S. R. Manalis. Weighing of biomolecules, single cells and single nanoparticles in fluid. Nature, 446(7139):1066-1069, 2007.

[35] T. P. Burg and S. R. Manalis. Suspended microchannel resonators for biomolecular detection. Applied Physics Letters, 83(13):2698-2700, 2003.

[36] T. P. Burg, A. R. Mirza, N. Milovic, C. H. Tsau, G. A. Popescu, J. S. Foster, and S. R. Manalis. Vacuum-packaged suspended microchannel resonant mass sensor for biomolecular detection. Journal of Microelectromechanical Systems, 15(6):1466-1476, 2006.

[37] T. P. Burg, J. E. Sader, and S. R. Manalis. Nonmonotonic Energy Dissipation in Microfluidic Resonators. Physical Review Letters, 102:228103-228106, 2009.

[38] M. J. Burke and M. A. Rougvie. Cross-beta protein structures i. insulin fibrils. Biochemistry, 11(13):2435-2439, 1972.

[39] M. J. Cannon, A. D. Williams, R. Wetzel, and D. G. Myszka. Kinetic analysis of beta-amyloid fibril elongation. Analytical Biochemistry, 328(1):67-75, 2004.

[40] A. Carre, W. Birch, and V. Lacarriere. Glass substrates modified with organosilanes for DNA immobilization, volume 4 of Silanes and Other Coupling Agents. 2007.

[41] N. Carulla, G. L. Caddy, D. R. Hall, J. Zurdo, M. Gairi, M. Feliz, E. Giralt, C. V. Robinson, and C. M. Dobson. Molecular recycling within amyloid fibrils. Nature, 436(7050):554-558, 2005.

[42] E. Chatani, Y. H. Lee, H. Yagi, Y. Yoshimura, H. Naiki, and Y. Goto. Ultrasonication-dependent production and breakdown lead to minimum-sized amyloid fibrils. Proceedings of the National Academy of Sciences of the United States of America, 106(27):11119-11124, 2009.

[43] B. Chen, K. R. Thurber, F. Shewmaker, R. B. Wickner, and R. Tycko. Measurement of amyloid fibril mass-per-length by tilted-beam transmission electron microscopy. Proc Natl Acad Sci U S A, 106(34):14339-44, 2009. 
[44] R. F. Chen and J. R. Knutson. Mechanism of fluorescence concentration quenching of carboxyfluorescein in liposomes - energy-transfer to nonfluorescent dimers. Analytical Biochemistry, 172(1):61-77, 1988.

[45] F. Chiti and C. M. Dobson. Amyloid formation by globular proteins under native conditions. Nature Chemical Biology, 5(1):15-22, 2009.

[46] K. Chitre, Q. Yang, T. O. Salami, S. R. Oliver, and J. Cho. Microstructure and mechanical properties of ceramic/self-assembled monolayer bilayer coatings. Journal of Electronic Materials, 34(5):528-533, 2005.

[47] S. Choi, M. Goryll, L. Y. M. Sin, P. K. Wong, and J. Chae. Microfluidicbased biosensors toward point-of-care detection of nucleic acids and proteins. Microfluidics and Nanofluidics, 10(2):231-247, 2011.

[48] S. K. Chu, M. Hsu, W. C. Ku, C. Y. Tu, Y. T. Tseng, W. K. Lau, R. Y. Yan, J. T. Ma, and C. M. Tzeng. Synergistic effects of epoxy- and amine-silanes on microarray dna immobilization and hybridization. Biochemical Journal, 374:625-632, 2003.

[49] R. Chunara, M. Godin, S. M. Knudsen, and S. R. Manalis. Mass-based readout for agglutination assays. Applied Physics Letters, 91(19), 2007.

[50] J. Clayton. Go with the microflow. Nature Methods, 2(8):621-627, 2005.

[51] G. W. Cline and S. B. Hanna. Kinetics and mechanisms of the aminolysis of nhydroxysuccinimide esters in aqueous buffers. Journal of Organic Chemistry, 53(15):3583-3586, 1988.

[52] F. E. Cohen and J. W. Kelly. Therapeutic approaches to protein-misfolding diseases. Nature, 426(6968):905-909, 2003.

[53] S. I. A. Cohen, M. Vendruscolo, C. M. Dobson, and T. P. J. Knowles. From macroscopic measurements to microscopic mechanisms of protein aggregation. Journal of Molecular Biology, 421(2-3):160-171, 2012.

[54] S. R. Collins, A. Douglass, R. D. Vale, and J. S. Weissman. Mechanism of prion propagation: Amyloid growth occurs by monomer addition. Plos Biology, 2(10):1582-1590, 2004.

[55] K. Cottier, M. Wiki, G. Voirin, H. Gao, and R. E. Kunz. Label-free highly sensitive detection of (small) molecules by wavelength interrogation of integrated optical chips. Sensors and Actuators B-Chemical, 91(1-3):241-251, 2003. 
[56] W. G. Cox, M. P. Beaudet, C. Ying, S. Yue, X. Chen, V. L. Singer, J. M. Beechem, and R. P. Haugland. A new class of fluorescent dyes: Alexa fluor 555 and alexa fluor 647 dyes are spectrally identical to cy3 and cy5 dyes, yet produce much brighter dna probes. American Journal of Human Genetics, 69(4):337-337, 2001.

[57] N. Cremades, S. I. A. Cohen, E. Deas, A. Y. Abramov, A. Y. Chen, A. Orte, M. Sandal, R. W. Clarke, P. Dunne, F. A. Aprile, C. W. Bertoncini, N. W. Wood, T. P. J. Knowles, C. M. Dobson, and D. Klenerman. Direct observation of the interconversion of normal and toxic forms of alpha-synuclein. Cell, 149(5):1048-1059, 2012.

[58] F. Darain, P. Yager, K. L. Gan, and S. C. Tjin. On-chip detection of myoglobin based on fluorescence. Biosensors E Bioelectronics, 24(6):1744-1750, 2009.

[59] T. J. Davis, D. D. Soto-Ortega, J. A. Kotarek, F. J. Gonzalez-Velasquez, K. Sivakumar, L. Y. Wu, Q. Wang, and M. A. Moss. Comparative study of inhibition at multiple stages of amyloid-beta self-assembly provides mechanistic insight. Molecular Pharmacology, 76(2):405-413, 2009.

[60] G. Demirel, M. Cakmak, T. Caykara, and S. Ellialtioglu. Chemisorption of 3aminopropyltrimethoxysilane on si(001)-(2 x 2). Journal of Physical Chemistry C, 111(41):15020-15025, 2007.

[61] P. Dextras, T. P. Burg, and S. R. Manalis. Integrated measurement of the mass and surface charge of discrete microparticles using a suspended microchannel resonator. Analytical Chemistry, 81(11):4517-4523, 2009.

[62] P. Dextras, K. R. Payer, T. P. Burg, W. J. Shen, Y. C. Wang, J. Y. Han, and S. R. Manalis. Fabrication and characterization of an integrated microsystem for protein preconcentration and sensing. Journal of Microelectromechanical Systems, 20(1):221-230, 2011.

[63] F. E. Dische, C. Wernstedt, G. T. Westermark, P. Westermark, M. B. Pepys, J. A. Rennie, S. G. Gilbey, and P. J. Watkins. Insulin as an amyloid-fibril protein at sites of repeated insulin injections in a diabetic patient. Diabetologia, 31(3):158-161, 1988.

[64] P. S. Dittrich and A. Manz. Lab-on-a-chip: microfluidics in drug discovery. Nature Reviews Drug Discovery, 5(3):210-218, 2006.

[65] C. M. Dobson. Protein misfolding, evolution and disease. Trends in Biochemical Sciences, 24(9):329-332, 1999. 
[66] C. M. Dobson. Protein folding and misfolding. Nature, 426(6968):884-890, 2003.

[67] A Dodge, K Fluri, E Verpoorte, and NF de Rooij. Electrokinetically driven microfluidic chips with surface-modified chambers for heterogeneous immunoassays. Analytical Chemistry, 73(14):3400-3409, 2001.

[68] Y. Dong, K. S. Phillips, and Q. Cheng. Immunosensing of staphylococcus enterotoxin b (seb) in milk with pdms microfluidic systems using reinforced supported bilayer membranes (r-sbms). Lab on a Chip, 6(5):675-681, 2006.

[69] B. Dorvel, B. Reddy, I. Block, P. Mathias, S. E. Clare, B. Cunningham, D. E. Bergstrom, and R. Bashir. Vapor-phase deposition of monofunctional alkoxysilanes for sub-nanometer-level biointerfacing on silicon oxide surfaces. Advanced Functional Materials, 20(1):87-95, 2010.

[70] E. D. Eanes and G. G. Glenner. X-ray diffraction studies on amyloid filaments. J Histochem Cytochem, 16(11):673-7, 1968.

[71] J. El-Ali, P. K. Sorger, and K. F. Jensen. Cells on chips. Nature, 442(7101):403-411, 2006.

[72] G. Elender, M. Kuhner, and E. Sackmann. Functionalisation of si/sio2 and glass surfaces with ultrathin dextran films and deposition of lipid bilayers. Biosensors \&3 Bioelectronics, 11(6-7):565-577, 1996.

[73] J. Emsley. Very strong hydrogen-bonding. Chemical Society Reviews, 9(1):91$124,1980$.

[74] W. P. Esler, A. M. Felix, E. R. Stimson, M. J. Lachenmann, J. R. Ghilardi, Y. A. Lu, H. V. Vinters, P. W. Mantyh, J. P. Lee, and J. E. Maggio. Activation barriers to structural transition determine deposition rates of alzheimer's disease a beta amyloid. Journal of Structural Biology, 130(2-3):174-183, 2000.

[75] E. Eteshola and D. Leckband. Development and characterization of an elisa assay in pdms microfluidic channels. Sensors and Actuators B-Chemical, 72(2):129-133, 2001.

[76] M. Fandrich. Absolute correlation between lag time and growth rate in the spontaneous formation of several amyloid-like aggregates and fibrils. Journal of Molecular Biology, 365(5):1266-1270, 2007.

[77] GS Fiorini and DT Chiu. Disposable microfluidic devices: fabrication, function, and application. Biotechniques, 38(3):429-446, MAR 2005. 
[78] A. W. P. Fitzpatrick, G. T. Debelouchina, M. J. Bayro, D. K. Clare, M. A. Caporini, V. S. Bajaj, C. P. Jaroniec, L. C. Wang, V. Ladizhansky, S. A. Muller, C. E. MacPhee, C. A. Waudby, H. R. Mott, A. De Simone, T. P. J. Knowles, H. R. Saibil, M. Vendruscolo, E. V. Orlova, R. G. Griffin, and C. M. Dobson. Atomic structure and hierarchical assembly of a cross-beta amyloid fibril. Proceedings of the National Academy of Sciences of the United States of America, 110(14):5468-5473, 2013.

[79] S. Flink, F. C. J. M. van Veggel, and D. N. Reinhoudt. Functionalization of self-assembled monolayers on glass and oxidized silicon wafers by surface reactions. Journal of Physical Organic Chemistry, 14(7):407-415, 2001.

[80] V. Fodera, M. Groenning, V. Vetri, F. Librizzi, S. Spagnolo, C. Cornett, L. Olsen, M. van de Weert, and M. Leone. Thioflavin t hydroxylation at basic ph and its effect on amyloid fibril detection. Journal of Physical Chemistry B, 112(47):15174-15181, 2008.

[81] Juergen Fritz. Cantilever biosensors. Analyst, 133(7):855-863, 2008.

[82] M. J. Fuerstman, A. Lai, M. E. Thurlow, S. S. Shevkoplyas, H. A. Stone, and G. M. Whitesides. The pressure drop along rectangular microchannels containing bubbles. Lab on a Chip, 7(11):1479-1489, 2007.

[83] T. Fukuma, M. Kimura, K. Kobayashi, K. Matsushige, and H. Yamada. Development of low noise cantilever deflection sensor for multienvironment frequency-modulation atomic force microscopy. Review of Scientific Instruments, 76(5), 2005.

[84] D. P. Funeriu, J. Eppinger, L. Denizot, M. Miyake, and J. Miyake. Enzyme family-specific and activity-based screening of chemical libraries using enzyme microarrays. Nature Biotechnology, 23(5):622-627, 2005.

[85] V. Gauvreau, P. Chevallier, K. Vallieres, E. Petitclerc, R. C. Gaudreault, and G. Laroche. Engineering surfaces for bioconjugation: Developing strategies and quantifying the extent of the reactions. Bioconjugate Chemistry, 15(5):1146-1156, 2004.

[86] E. Gazit. Mechanisms of amyloid fibril self-assembly and inhibition. Febs Journal, 272(23):5971-5978, 2005.

[87] A. J. Geddes, K. D. Parker, E. D. Atkins, and E. Beighton. "cross-beta" conformation in proteins. Journal of Molecular Biology, 32(2):343-58, 1968. 
[88] T. Gervais and K. F. Jensen. Mass transport and surface reactions in microfluidic systems. Chemical Engineering Science., 61(4):1102-1121, 2006.

[89] K. Giger, R. P. Vanam, E. Seyrek, and P. L. Dubin. Suppression of insulin aggregation by heparin. Biomacromolecules, 9(9):2338-2344, 2008.

[90] J. T. Giurleo, X. L. He, and D. S. Talaga. beta-lactoglobulin assembles into amyloid through sequential aggregated intermediates. Journal of Molecular Biology, 381(5):1332-1348, 2008.

[91] N. R. Glass, R. Tjeung, P. Chan, L. Y. Yeo, and J. R. Friend. Organosilane deposition for microfluidic applications. Biomicrofluidics, 5(3), 2011.

[92] M. Gobbi, L. Colombo, M. Morbin, G. Mazzoleni, E. Accardo, M. Vanoni, E. Del Favero, L. Cantu, D. A. Kirschner, C. Manzoni, M. Beeg, P. Ceci, P. Ubezio, G. Forloni, F. Tagliavini, and M. Salmona. Gerstmann-strarusslerscheinker disease amyloid protein polymerizes according to the "dock-andlock" model. Journal of Biological Chemistry, 281(2):843-849, 2006.

[93] M. Godin, A. K. Bryan, T. P. Burg, K. Babcock, and S. R. Manalis. Measuring the mass, density, and size of particles and cells using a suspended microchannel resonator. Applied Physics Letters, 91(12), 2007.

[94] M. Godin, F. F. Delgado, S. M. Son, W. H. Grover, A. K. Bryan, A. Tzur, P. Jorgensen, K. Payer, A. D. Grossman, M. W. Kirschner, and S. R. Manalis. Using buoyant mass to measure the growth of single cells. Nature Methods, 7(5):387-U70, 2010.

[95] M. Goedert. Alpha-synuclein and neurodegenerative diseases. Nature Reviews Neuroscience, 2(7):492-501, 2001.

[96] C. Goldsbury, P. Frey, V. Olivieri, U. Aebi, and S. A. Muller. Multiple assembly pathways underlie amyloid-beta fibril polymorphisms. Journal of Molecular Biology, 352(2):282-98, 2005.

[97] C. Goldsbury, J. Kistler, U. Aebi, T. Arvinte, and G. J. S. Cooper. Watching amyloid fibrils grow by time-lapse atomic force microscopy. Journal of Molecular Biology, 285(1):33-39, 1999.

[98] J. D. Green, L. Kreplak, C. Goldsbury, X. L. Blatter, M. Stolz, G. S. Cooper, A. Seelig, J. Kist-Ler, and U. Aebi. Atomic force microscopy reveals defects within mica supported lipid bilayers induced by the amyloidogenic human amylin peptide. Journal of Molecular Biology, 342(3):877-887, 2004. 
[99] W. H. Grover, A. K. Bryan, M. Diez-Silva, S. Suresh, J. M. Higgins, and S. R. Manalis. Measuring single-cell density. Proc Natl Acad Sci U S A, 108(27):10992-6, 2011.

[100] T. E. Gunter, K. K. Gunter, S. S. Sheu, and C. E. Gavin. Mitochondrial calcium-transport - physiological and pathological relevance mitochondrial calcium-transport. American Journal OF Physiology, 267(2):C313-C339, 1994.

[101] G. Habicht, C. Haupt, R. P. Friedrich, P. Hortschansky, C. Sachse, J. Meinhardt, K. Wieligmann, G. P. Gellermann, M. Brodhun, J. Gotz, K. J. Halbhuber, C. Rocken, U. Horn, and M. Fandrich. Directed selection of a conformational antibody domain that prevents mature amyloid fibril formation by stabilizing a beta protofibrils. Proceedings of the National Academy of Sciences of the United States of America, 104(49):19232-19237, 2007.

[102] T. Hard and C. Lendel. Inhibition of amyloid formation. Journal of Molecular Biology, 421(4-5):441-465, 2012.

[103] J. Hardy and D. J. Selkoe. Medicine - the amyloid hypothesis of alzheimer's disease: Progress and problems on the road to therapeutics. Science, 297(5580):353-356, 2002.

[104] F. U. Hartl and M. Hayer-Hartl. Converging concepts of protein folding in vitro and in vivo. Nature Structural \&f Molecular Biology, 16(6):574-581, 2009.

[105] K. Hasegawa, K. Ono, M. Yamada, and H. Naiki. Kinetic modeling and determination of reaction constants of alzheimer's beta-amyloid fibril extension and dissociation using surface plasmon resonance. Biochemistry, 41(46):1348913498, 2002.

[106] M. Hashimoto, H. Kaji, M. E. Kemppinen, and M. Nishizawa. Localized immobilization of proteins onto microstructures within a preassembled microfluidic device. Sensors and Actuators B-Chemical, 128(2):545-551, 2008.

[107] C. L. Heldt, S. Q. Zhang, and G. Belfort. Asymmetric amyloid fibril elongation: A new perspective on a symmetric world. Proteins-Structure Function and Bioinformatics, 79(1):92-98, 2011.

[108] G. T. Hermanson. Bioconjugate techniques, 2nd edition. Bioconjugate Techniques, 2nd Edition, pages 1-1202, 2008. 
[109] E. K. Hill, B. Krebs, D. G. Goodall, G. J. Howlett, and D. E. Dunstan. Shear flow induces amyloid fibril formation. Biomacromolecules, 7(1):10-13, 2006.

[110] D. B. Holmes and Vermeule.Jr. Velocity profiles in ducts with rectangular cross sections. Chemical Engineering Science, 23(7):717-\&, 1968.

[111] M. Hoshino, H. Katou, Y. Hagihara, K. Hasegawa, H. Naiki, and Y. Goto. Mapping the core of the beta(2)-microglobulin amyloid fibril by $\mathrm{h} / \mathrm{d}$ exchange. Nature Structural Biology, 9(5):332-336, 2002.

[112] K. Hosokawa, M. Omata, K. Sato, and M. Maeda. Power-free sequential injection for microchip immunoassay toward point-of-care testing. Lab on a Chip, 6(2):236-241, 2006.

[113] W. G. Hoyer, D. Cherny, V. Subramaniam, and T. M. Jovin. Rapid selfassembly of alpha-synuclein observed by in situ atomic force microscopy. Journal of Molecular Biology, 340(1):127-139, 2004.

[114] C. Huang, Y. Li, and S Jiang. Zwitterionic polymer-based platform with twolayer architecture for ultra low fouling and high protein loading. Analytical Chemistry, 84:3440-3445, 2012.

[115] S. A. Hudson, H. Ecroyd, T. W. Kee, and J. A. Carver. The thioflavin t fluorescence assay for amyloid fibril detection can be biased by the presence of exogenous compounds. Febs Journal, 276(20):5960-5972, 2009.

[116] M. I. Ivanova, S. A. Sievers, M. R. Sawaya, J. S. Wall, and D. Eisenberg. Molecular basis for insulin fibril assembly. Proceedings of the National Academy of Sciences of the United States of America, 106(45):18990-18995, 2009.

[117] J. A. Jackman, W. Knoll, and N. J. Cho. Biotechnology applications of tethered lipid bilayer membranes. Materials, 5(12):2637-2657, 2012.

[118] T. R. Jahn and S. E. Radford. Folding versus aggregation: Polypeptide conformations on competing pathways. Archives of Biochemistry and Biophysics, 469(1):100-117, 2008.

[119] R. Jansen, W. Dzwolak, and R. Winter. Amyloidogenic self-assembly of insulin aggregates probed by high resolution atomic force microscopy. Biophysical Journal, 88(2):1344-1353, 2005. 
[120] J. L. Jimenez, E. J. Nettleton, M. Bouchard, C. V. Robinson, C. M. Dobson, and H. R. Saibil. The protofilament structure of insulin amyloid fibrils. Proceedings of the National Academy of Sciences of the United States of America, 99(14):9196-9201, 2002.

[121] B. Johnsson, S. Lofas, and G. Lindquist. Immobilization of proteins to a carboxymethyldextran-modified gold surface for biospecific interaction analysis in surface-plasmon resonance sensors. Analytical Biochemistry, 198(2):268$277,1991$.

[122] C. Jonsson, M. Aronsson, G. Rundstrom, C. Pettersson, I. Mendel-Hartvig, J. Bakker, E. Martinsson, B. Liedberg, B. MacCraith, O. Ohman, and J. Melin. Silane-dextran chemistry on lateral flow polymer chips for immunoassays. Lab on a Chip, 8(7):1191-1197, 2008.

[123] P. Juszczyk, A. S. Kolodziejczyk, and Z. Grzonka. Circular dichroism and aggregation studies of amyloid beta (11-8) fragment and its variants. Acta Biochim Pol, 52(2):425-31, 2005.

[124] M. Katakam and A. K. Banga. Aggregation of insulin and its prevention by carbohydrate excipients. PDA J Pharm Sci Technol, 49(4):160-5, 1995.

[125] B. Katzbauer, M. Narodoslawsky, and A. Moser. Classification-system for immobilization techniques. Bioprocess Engineering, 12(4):173-179, 1995.

[126] A. J. Keefe, K. B. Caldwell, Nowinski A. K., A. T. White, and S. Jiang. Screening nonspecific interactions of peptides without background interference. Biomaterials, 34(8):1871-1877, 2013.

[127] J. W. Kelly. Mechanisms of amyloidogenesis. Nature Structural Biology, $7(10): 824-826,2000$.

[128] S. M. Kelly, T. J. Jess, and N. C. Price. How to study proteins by circular dichroism. Biochimica Et Biophysica Acta-Proteins and Proteomics, 1751(2):119-139, 2005.

[129] R. Khurana, C. Coleman, C. Ionescu-Zanetti, S. A. Carter, V. Krishna, R. K. Grover, R. Roy, and S. Singh. Mechanism of thioflavin t binding to amyloid fibrils. Journal of Structural Biology, 151(3):229-238, 2005.

[130] R. Khurana, C. Ionescu-Zanetti, M. Pope, J. Li, L. Nielson, M. RamirezAlvarado, L. Regan, A. L. Fink, and S. A. Carter. A general model for amyloid fibril assembly based on morphological studies using atomic force microscopy. Biophysical Journal, 85(2):1135-1144, 2003. 
[131] R. Khurana, V. N. Uversky, L. Nielsen, and A. L. Fink. Is congo red an amyloid-specific dye? Journal of Biological Chemistry, 276(25):22715-22721, 2001.

[132] D. Kim, K. Karns, S. Q. Tia, M. He, and A. E. Herr. Electrostatic protein immobilization using charged polyacrylamide gels and cationic detergent microfluidic western blotting. Anal Chem, 84(5):2533-40, 2012.

[133] Dohyun Kim and Amy E. Herr. Protein immobilization techniques for microfluidic assays. Biomicrofluidics, 7(4):041501, 2013.

[134] H. J. Kim, E. Chatani, Y. Goto, and S. R. Paik. Seed-dependent accelerated fibrillation of alpha-synuclein induced by periodic ultrasonication treatment. Journal of Microbiology and Biotechnology, 17(12):2027-2032, 2007.

[135] H. Y. Kim, M. K. Cho, D. Riedel, C. O. Fernandez, and M. Zweckstetter. Dissociation of amyloid fibrils of alpha-synuclein in supercooled water. Angewandte Chemie-International Edition, 47(27):5046-5048, 2008.

[136] R. Kisilevsky. Review: Amyloidogenesis-unquestioned answers and unanswered questions. Journal of Structural Biology, 130(2-3):99-108, 2000.

[137] T. P. J. Knowles and M. J. Buehler. Nanomechanics of functional and pathological amyloid materials. Nature Nanotechnology, 6(8):469-479, 2011.

[138] T. P. J. Knowles, W. M. Shu, G. L. Devlin, S. Meehan, S. Auer, C. M. Dobson, and M. E. Welland. Kinetics and thermodynamics of amyloid formation from direct measurements of fluctuations in fibril mass. Proceedings of the National Academy of Sciences of the United States of America, 104(24):10016-10021, 2007.

[139] E. H. Koo, P. T. Lansbury, and J. W. Kelly. Amyloid diseases: Abnormal protein aggregation in neurodegeneration. Proceedings of the National Academy of Sciences of the United States of America, 96(18):9989-9990, 1999.

[140] M. R. H. Krebs, E. H. C. Bromley, and A. M. Donald. The binding of thioflavin-t to amyloid fibrils: localisation and implications. Journal of Structural Biology, 149(1):30-37, 2005.

[141] J. Krenkova and F. Foret. Immobilized microfluidic enzymatic reactors. Electrophoresis, 25(21-22):3550-3563, 2004. 
[142] H. Kroth, A. Ansaloni, Y. Varisco, A. Jan, N. Sreenivasachary, N. RezaeiGhaleh, V. Giriens, S. Lohmann, M. P. Lopez-Deber, O. Adolfsson, M. Pihlgren, P. Paganetti, W. Froestl, L. Nagel-Steger, D. Willbold, T. Schrader, M. Zweckstetter, A. Pfeifer, H. A. Lashuel, and A. Muhs. Discovery and structure activity relationship of small molecule inhibitors of toxic betaamyloid-42 fibril formation. Journal of Biological Chemistry, 287(41):3478634800, 2012.

[143] S. Kumar and J. Walter. Phosphorylation of amyloid beta (a beta) peptides - a trigger for formation of toxic aggregates in alzheimer's disease. Aging-Us, 3(8):803-812, 2011.

[144] L. Kunz and A. J. MacRobert. Intracellular photobleaching of 5,10,15,20tetrakis(m-hydroxyphenyl) chlorin (foscan $((\mathrm{r}))$ ) exhibits a complex dependence on oxygen level and fluence rate. Photochemistry and Photobiology, $75(1): 28-35,2002$.

[145] D. G. Kurth and T. Bein. Quantification of the reactivity of 3-aminopropyltriethoxysilane monolayers with the quartz-crystal microbalance. Angewandte Chemie-International Edition in English, 31(3):336-338, 1992.

[146] Y. Kusumoto, A. Lomakin, D. B. Teplow, and G. B. Benedek. Temperature dependence of amyloid beta-protein fibrillization. Proceedings of the National Academy of Sciences of the United States of America, 95(21):12277-12282, 1998.

[147] K. B. Lam, E. F. Irwin, K. E. Healy, and L. W. Lin. Bioelectrocatalytic selfassembled thylakoids for micro-power and sensing applications. Sensors and Actuators B-Chemical, 117(2):480-487, 2006.

[148] J. L. Larson, E. Ko, and A. D. Miranker. Direct measurement of islet amyloid polypeptide fibrillogenesis by mass spectrometry. Protein Science, 9(2):427$431,2000$.

[149] C. Y. Lee, P. Gong, G. M. Harbers, D. W. Grainger, D. G. Castner, and L. J. Gamble. Surface coverage and structure of mixed dna/alkylthiol monolayers on gold: Characterization by xps, nexafs, and fluorescence intensity measurements. Analytical Chemistry, 78(10):3316-3325, 2006.

[150] H. Lee, S. M. Dellatore, W. M. Miller, and P. B. Messersmith. Mussel-inspired surface chemistry for multifunctional coatings. Science, 318(5849):426-430, 2007. 
[151] I. Lee and J. Lee. Measurement of mixing ratio and volume change of ethanolwater binary mixtures using suspended microchannel resonators. 2012 Ieee Sensors Proceedings, pages 574-576, 2012.

[152] I. Lee, K. Park, and J. Lee. Precision density and volume contraction measurements of ethanol-water binary mixtures using suspended microchannel resonators. Sensors and Actuators a-Physical, 194:62-66, 2013.

[153] J. Lee, R. Chunara, W. Shen, K. Payer, K. Babcock, T. P. Burg, and S. R. Manalis. Suspended microchannel resonators with piezoresistive sensors. Lab on a Chip, 11(4):645-651, 2011.

[154] Y. S. Lee and M. Mrksich. Protein chips: from concept to practice. Trends in Biotechnology, 20(12):S14-S18, 2002.

[155] C. Lendel, B. Bolognesi, A. Wahlstrom, C. M. Dobson, and A. Graslund. Detergent-like interaction of congo red with the amyloid beta peptide. Biochemistry, 49(7):1358-1360, 2010.

[156] M. L. Lesaicherre, M. Uttamchandani, G. Y. J. Chen, and S. Q. Yao. Developing site-specific immobilization strategies of peptides in a microarray. Bioorganic \& Medicinal Chemistry Letters, 12(16):2079-2083, 2002.

[157] H. LeVine. Stopped-flow kinetics reveal multiple phases of thioflavin t binding to alzheimer beta(1-40) amyloid fibrils. Archives of Biochemistry and Biophysics, 342(2):306-316, 1997.

[158] M. Levy-Sakin, M. Shreberk, Y. Daniel, and E. Gazit. Targeting insulin amyloid assembly by small aromatic molecules toward rational design of aggregation inhibitors. Islets, 1(3):210-215, 2009.

[159] D. R. Lide. CRC Handbook of Chemistry and Physics, volume 94th edition. 2013.

[160] C. C. Lin, J. H. Wang, H. W. Wu, and G. B. Lee. Microfluidic immunoassays. Jala, 15(3):253-274, 2010.

[161] V. Linder, E. Verpoorte, W. Thormann, N. F. de Rooij, and M. Sigrist. Surface biopassivation of replicated poly(dimethylsiloxane) microfluidic channels and application to heterogeneous immunoreaction with on-chip fluorescence detection. Anal Chem, 73(17):4181-4189, 2001. 
[162] C. M. Liu, R. P. Liang, X. N. Wang, J. W. Wang, and J. D. Qiu. A versatile polydopamine platform for facile preparation of protein stationary phase for chip-based open tubular capillary electrochromatography enantioseparation. Journal of Chromatography A, 1294:145-151, 2013.

[163] J. T. Liu, C. J. Chen, T. Ikoma, T. Yoshioka, J. S. Cross, S. J. Chang, J. Z. Tsai, and J. Tanaka. Surface plasmon resonance biosensor with high anti-fouling ability for the detection of cardiac marker troponin t. Analytica Chimica Acta, 703(1):80-86, 2011.

[164] R. Lourenco and M. E. Camilo. Magnesium: physiological and clinical relevance.1: homeostasis and alterations in the metabolism of magnesium. Acta Medical Port, 13(4):211-220, 2000.

[165] H. Lu, L. Y. Koo, W. C. M. Wang, D. A. Lauffenburger, L. G. Griffith, and K. F. Jensen. Microfluidic shear devices for quantitative analysis of cell adhesion. Analytical Chemistry, 76(18):5257-5264, 2004.

[166] S. R. Kain M. Chalfie. Green fluorescence protein. Wiley-Interscience, 2006.

[167] R. Malik and I. Roy. Probing the mechanism of insulin aggregation during agitation. International Journal of Pharmaceutics, 413(1-2):73-80, 2011.

[168] K. E. Marshall and L. C. Serpell. Insights into the structure of amyloid fibrils. The Open Biology Journal, 2:185-192, 2009.

[169] F. Massi and J. E. Straub. Energy landscape theory for alzheimer's amyloid beta-peptide fibril elongation. Proteins-Structure Function and Genetics, 42(2):217-229, 2001.

[170] M. Mauro, E. F. Craparo, A. Podesta, D. Bulone, R. Carrotta, V. Martorana, G. Tiana, and P. L. San Biagio. Kinetics of different processes in human insulin amyloid formation. Journal of Molecular Biology, 366(1):258-274, 2007.

[171] G. Maurstad, M. Prass, L. C. Serpell, and P. Sikorski. Dehydration stability of amyloid fibrils studied by afm. European Biophysics Journal with Biophysics Letters, 38(8):1135-1140, 2009.

[172] F. Meersman, C. M. Dobson, and K. Heremans. Protein unfolding, amyloid fibril formation and configurational energy landscapes under high pressure conditions. Chemical Society Reviews, 35(10):908-917, 2006. 
[173] E. Metwalli, D. Haines, O. Becker, S. Conzone, and C. G. Pantano. Surface characterizations of mono-, di-, and tri-aminosilane treated glass substrates. Journal of Colloid and Interface Science, 298(2):825-831, 2006.

[174] I. Migneault, C. Dartiguenave, M. J. Bertrand, and K. C. Waldron. Glutaraldehyde: behavior in aqueous solution, reaction with proteins, and application to enzyme crosslinking. Biotechniques, 37(5):790-+, 2004.

[175] E. Milkani, C. R. Lambert, and W. G. McGimpsey. Direct detection of acetylcholinesterase inhibitor binding with an enzyme-based surface plasmon resonance sensor. Analytical Biochemistry, 408(2):212-219, 2011.

[176] L. N. Mironova, A. I. Goginashvili, and M. D. Ter-Avanesyan. Biological functions of amyloids: Facts and hypotheses. Molecular Biology, 42(5):710$719,2008$.

[177] M. M. Modena, Y. Wang, D. Riedel, and T.P. Burg. Resolution enhancement of suspended microchannel resonators for weighing of biomolecular complexes in solution. Lab on a Chip, 2013.

[178] J. Mohanty, S. D. Choudhury, H. Pal, and A. C. Bhasikuttan. Early detection of insulin fibrillation: a fluorescence lifetime assay to probe the pre-fibrillar regime. Chemical Communications, 48(18):2403-2405, 2012.

[179] J. H. Moon, J. H. Kim, K. Kim, T. H. Kang, B. Kim, C. H. Kim, J. H. Hahn, and J. W. Park. Absolute surface density of the amine group of the aminosilylated thin layers: Ultraviolet-visible spectroscopy, second harmonic generation, and synchrotron-radiation photoelectron spectroscopy study. Langmuir, 13(16):4305-4310, 1997.

[180] J. H. Moon, J. W. Shin, S. Y. Kim, and J. W. Park. Formation of uniform aminosilane thin layers: An imine formation to measure relative surface density of the amine group. Langmuir, 12(20):4621-4624, 1996.

[181] J. H. Moon, J. W. Shin, and J. W. Park. Self-assembly of aminosilane layers: Determination of surface density of the amine group through a reversible chemical reaction. Molecular Crystals and Liquid Crystals Science and Technology Section a-Molecular Crystals and Liquid Crystals, 294:483-486, 1997.

[182] Y. Nam, D. W. Branch, and B. C. Wheeler. Epoxy-silane linking of biomolecules is simple and effective for patterning neuronal cultures. Biosensors $\&$ Bioelectronics, 22(5):589-597, 2006. 
[183] R. Nelson and D. Eisenberg. Recent atomic models of amyloid fibril structure. Current Opinion in Structural Biology, 16(2):260-265, 2006.

[184] R. Nelson, M. R. Sawaya, M. Balbirnie, A. O. Madsen, C. Riekel, R. Grothe, and D. Eisenberg. Structure of the cross-beta spine of amyloid-like fibrils. Nature, 435(7043):773-778, 2005.

[185] A. Nematollahzadeh, A. Shojaei, M. J. Abdekhodaie, and B. Sellergren. Molecularly imprinted polydopamine nano-layer on the pore surface of porous particles for protein capture in hplc column. Journal of Colloid and Interface Science, 404:117-126, 2013.

[186] A. H. C. Ng, U. Uddayasankar, and A. R. Wheeler. Immunoassays in microfluidic systems. Analytical and Bioanalytical Chemistry, 397(3):991-1007, 2010 .

[187] L. Nielsen, S. Frokjaer, J. Brange, V. N. Uversky, and A. L. Fink. Probing the mechanism of insulin fibril formation with insulin mutants. Biochemistry, 40(28):8397-8409, 2001.

[188] L. Nielsen, R. Khurana, A. Coats, S. Frokjaer, J. Brange, S. Vyas, V. N. Uversky, and A. L. Fink. Effect of environmental factors on the kinetics of insulin fibril formation: Elucidation of the molecular mechanism. Biochemistry, 40(20):6036-6046, 2001.

[189] M. R. Nilsson. Techniques to study amyloid fibril formation in vitro. Methods, 34(1):151-160, 2004.

[190] S. Noel, B. Liberelle, L. Robitaille, and G. De Crescenzo. Quantification of primary amine groups available for subsequent biofunctionalization of polymer surfaces. Bioconjugate Chemistry, 22(8):1690-1699, 2011.

[191] A. Noormagi, J. Gavrilova, J. Smirnova, V. Tougu, and P. Palumaa. Zn(ii) ions co-secreted with insulin suppress inherent amyloidogenic properties of monomeric insulin. Biochemical Journal, 430:511-518, 2010.

[192] A. Noormai, K. Primar, V. Tougu, and P. Palumaa. Interference of lowmolecular substances with the thioflavin-t fluorescence assay of amyloid fibrils. Journal of Peptide Science, 18(1):59-64, 2012.

[193] C. Nordstedt, J. Naslund, L. O. Tjernberg, A. R. Karlstrom, J. Thyberg, and L. Terenius. The alzheimer a-beta-peptide develops protease resistance in association with its polymerization into fibrils. Journal of Biological Chemistry, 269(49):30773-30776, 1994. 
[194] S. J. Oh, S. J. Cho, C. O. Kim, and J. W. Park. Characteristics of dna microarray fabricated on the various aminosilane layers. Langmuir, 18:17641769, 2002.

[195] S. Ohnishi and K. Takano. Amyloid fibrils from the viewpoint of protein folding. Cellular and Molecular Life Sciences, 61(5):511-524, 2004.

[196] H. Okuno, K. Mori, T. Jitsukawa, H. Inoue, and S. Chiba. Convenient method for monitoring a beta aggregation by quartz-crystal microbalance. Chemical Biology \& Drug Design, 68(5):273-275, 2006.

[197] B. O'Nuallain, S. Shivaprasad, I. Kheterpal, and R. Wetzel. Thermodynamics of a beta(1-40) amyloid fibril elongation. Biochemistry, 44(38):12709-18, 2005.

[198] M. Oz, D. E. Lorke, and G. A. Petroianu. Methylene blue and alzheimer's disease. Biochemical Pharmacology, 78(8):927-932, 2009.

[199] S. Pal, M. J. Kim, and J. M. Song. Quantitation of surface coverage of oligonucleotides bound to chip surfaces: a fluorescence-based approach using alkaline phosphatase digestion. Lab on a Chip, 8(8):1332-1341, 2008.

[200] N. Panchuk-Voloshina, R.P. Haugland, J. Bishop-Stewart, M.K. Bhalgat, P.J. Millard, F. Mao, W.Y. Leung, and R.P. Haugland. Alexa dyes, a series of new fluorescent dyes that yield exceptionally bright, photostable conjugates. Journal of Histochemistry \&3 Cytochemistry, 47(9):1179-1188, 1999.

[201] MD PARTIS, DG GRIFFITHS, GC ROBERTS, and RB BEECHEY. CROSS-LINKING OF PROTEIN BY OMEGA-MALEIMIDO ALKANOYL N-HYDROXYSUCCINIMIDO ESTERS. JOURNAL OF PROTEIN CHEMISTRY, 2(3):263-277, 1983.

[202] R. F. Pasternack, E. J. Gibbs, S. Sibley, L. Woodard, P. Hutchinson, J. Genereux, and K. Kristian. Formation kinetics of insulin-based amyloid gels and the effect of added metalloporphyrins. Biophysical Journal, 90(3):1033$1042,2006$.

[203] A. R. Patel, D. Lau, and J. Liu. Quantification and characterization of micrometer and submicrometer subvisible particles in protein therapeutics by use of a suspended microchannel resonator. Analytical Chemistry, 84(15):6833-6840, 2012.

[204] M. R. Paul, M. T. Clark, and M. C. Cross. The stochastic dynamics of micron and nanoscale elastic cantilevers in fluid: fluctuations from dissipation. Namotechnology, 17(17):4502-4513, 2006. 
[205] Jeppe T. Pedersen and Niels H. H. Heegaard. Analysis of protein aggregation in neurodegenerative disease. Analytical Chemistry, 2013.

[206] M. B. Pepys. Pathogenesis, diagnosis and treatment of systemic amyloidosis. Philosophical Transactions of the Royal Society B-Biological Sciences, 356(1406):203-210, 2001.

[207] F. Persson, J. Fritzsche, K. U. Mir, M. Modesti, F. Westerlund, and J. O. Tegenfeldt. Lipid-based passivation in nanofluidics. Nano Letters, 12(5):2260$2265,2012$.

[208] D. S. Peterson. Solid supports for micro analytical systems. Lab on a Chip, 5(2):132-139, 2005.

[209] K. C. Popat and T. A. Desai. Poly(ethylene glycol) interfaces: an approach for enhanced performance of microfluidic systems. Biosensors \& Bioelectronics, 19(9):1037-1044, 2004.

[210] J. Poznariski, J. Szymariski, T. Basinska, S. Slomkowski, and W. YZielenkiewicz. Aggregation of aqueous lysozyme solutions followed by dynamic light scattering and $1 \mathrm{~h} n \mathrm{~nm}$ spectroscopy. Journal of Molecular Liquids, 121:21-26, 2005.

[211] S. B. Prusiner, M. P. Mckinley, K. A. Bowman, D. C. Bolton, P. E. Bendheim, D. F. Groth, and G. G. Glenner. Scrapie prions aggregate to form amyloid-like birefringent rods. Cell, 35(2):349-358, 1983.

[212] E. Pryor, J. A. Kotarek, M. A. Moss, and C. N. Hestekin. Monitoring insulin aggregation via capillary electrophoresis. International Journal of Molecular Sciences, 12(12):9369-9388, 2011.

[213] W. Qiang, W. M. Yau, Y. Q. Luo, M. P. Mattson, and R. Tycko. Antiparallel beta-sheet architecture in iowa-mutant beta-amyloid fibrils. Proceedings of the National Academy of Sciences of the United States of America, 109(12):44434448, 2012.

[214] D. C. Rambaldi, A. Zattoni, P. Reschiglian, R. Colombo, and E. De Lorenzi. In vitro amyloid a beta(1-42) peptide aggregation monitoring by asymmetrical flow field-flow fractionation with multi-angle light scattering detection. Analytical and Bioanalytical Chemistry, 394(8):2145-2149, 2009.

[215] I. Ramos, D. Fabris, W. Qi, E. J. Fernandez, and T. A. Good. Kinetic study of beta-amyloid residue accessibility using reductive alkylation and mass spectrometry. Biotechnology and Bioengineering, 104(1):181-192, 2009. 
[216] A. Revzin, E. Maverakis, and H. C. Chang. Biosensors for immune cell analysis-a perspective. Biomicrofluidics, 6(2), 2012.

[217] N. Rezaei-Ghaleh, M. Blackledge, and M. Zweckstetter. Intrinsically disordered proteins: From sequence and conformational properties toward drug discovery. Chembiochem, 13(7):930-950, 2012.

[218] S. S. Rogers, M. R. Krebs, E. H. Bromley, E. Van der Linden, and A. M. Donald. Optical microscopy of growing insulin amyloid spherulites on surfaces in vitro. Biophysical Journal, 90(3):1043-1054, 2006.

[219] S. S. Rogers, P. Venema, L. M. C. Sagis, E. van der Linden, and A. M. Donald. Measuring the length distribution of a fibril system: A flow birefringence technique applied to amyloid fibrils. Macromolecules, 38(7):2948-2958, 2005.

[220] C. A. Ross and M. A. Poirier. Protein aggregation and neurodegenerative disease. Nature Medicine, 10:10-17, 2004.

[221] L. A. Ruiz-Taylor, T. L. Martin, F. G. Zaugg, K. Witte, P. Indermuhle, S. Nock, and P. Wagner. Monolayers of derivatized poly(l-lysine)-grafted poly(ethylene glycol) on metal oxides as a class of biomolecular interfaces. Proceeding of the National Academy of Sciences of the United States of America, 98(3):852-7, 2001.

[222] F. Rusmini, Z. Y. Zhong, and J. Feijen. Protein immobilization strategies for protein biochips. Biomacromolecules, 8(6):1775-1789, 2007.

[223] A. Sandberg, L. M. Luheshi, S. Sollvander, T. P. de Barros, B. Macao, T. P. J. Knowles, H. Biverstal, C. Lendel, F. Ekholm-Petterson, A. Dubnovitsky, L. Lannfelt, C. M. Dobson, and T. Hard. Stabilization of neurotoxic alzheimer amyloid-beta oligomers by protein engineering. Proceedings of the National Academy of Sciences of the United States of America, 107(35):1559515600, 2010.

[224] H. Schift, S. Saxer, S. Park, C. Padeste, U. Pieles, and J. Gobrecht. Controlled co-evaporation of silanes for nanoimprint stamps. Namotechnology, 16(5):S171-S175, 2005.

[225] M. Schleeger, C. C. vandenAkker, T. Deckert-Gaudig, V. Deckert, K. P. Velikov, G. Koenderink, and M. Bonn. Amyloids: From molecular structure to mechanical properties. Polymer, 54(10):2473-2488, 2013. 
[226] K. Schult, A. Katerkamp, D. Trau, F. Grawe, K. Cammann, and M. Meusel. Disposable optical sensor chip for medical diagnostics: New ways in bioanalysis. Analytical Chemistry, 71(23):5430-5435, 1999.

[227] W. H. Scouten, J. H. T. Luong, and R. S. Brown. Enzyme or protein immobilization techniques for applications in biosensor design. Trends in Biotechnology, 13(5):178-185, 1995.

[228] C. Selvaggini, L. Degioia, L. Cantu, E. Ghibaudi, L. Diomede, F. Passerini, G. Forloni, O. Bugiani, F. Tagliavini, and M. Salmona. Molecular characteristics of a protease-resistant, amyloidogenic and neurotoxic peptide homologous to residues-106-126 of the prion protein. Biochemical and Biophysical Research Communications, 194(3):1380-1386, 1993.

[229] S. Seneff, G. Wainwright, and L. Mascitelli. Nutrition and alzheimer's disease: The detrimental role of a high carbohydrate diet. European Journal of Internal Medicine, 22(2):134-140, 2011.

[230] C. L. Shen, G. L. Scott, F. Merchant, and R. M. Murphy. Light-scattering analysis of fibril growth from the amino-terminal fragment beta(1-28) of betaamyloid peptide. Biophysical Journal, 65(6):2383-2395, 1993.

[231] S. P. Sibley, K. Sosinsky, L. E. Gulian, E. J. Gibbs, and R. F. Pasternack. Probing the mechanism of insulin aggregation with added metalloporphyrins. Biochemistry, 47(9):2858-2865, 2008.

[232] J. D. Sipe, M. D. Benson, J. N. Buxbaum, S. Ikeda, G. Merlini, M. J. M. Saraiva, and P. Westermark. Amyloid fibril protein nomenclature: 2010 recommendations from the nomenclature committee of the international society of amyloidosis. Amyloid-Journal of Protein Folding Disorders, 17(3-4):101-104, 2010 .

[233] J. D. Sipe and A. S. Cohen. Review: History of the amyloid fibril. Journal of Structural Biology, 130(2-3):88-98, 2000.

[234] V. Sivagnanam, B. Song, C. Vandevyver, and M. A. M. Gijs. On-chip immunoassay using electrostatic assembly of streptavidin-coated bead micropatterns. Anal Chem, 81(15):6509-6515, 2009.

[235] J. F. Smith, T. P. J. Knowles, C. M. Dobson, C. E. MacPhee, and M. E. Welland. Characterization of the nanoscale properties of individual amyloid fibrils. Proceedings of the National Academy of Sciences of the United States of America, 103(43):15806-15811, 2006. 
[236] S. Son, A. Tzur, Y. Weng, P. Jorgensen, J. Kim, M. W. Kirschner, and S. R. Manalis. Direct observation of mammalian cell growth and size regulation. Nature Methods, 9(9):910-913, 2012.

[237] L. L. Song, E. J. Hennink, I. T. Young, and H. J. Tanke. Photobleaching kinetics of fluorescein in quantitative fluorescence microscopy. Biophysical Journal, 68(6):2588-2600, 1995.

[238] M. Soos, A. S. Moussa, L. Ehrl, J. Sefcik, H. Wu, and M. Morbidelli. Effect of shear rate on aggregate size and morphology investigated under turbulent conditions in stirred tank. Journal of Colloid and Interface Science, 319(2):577-589, 2008.

[239] C. Soto. Unfolding the role of protein misfolding in neurodegenerative diseases. Nature Reviews Neuroscience, 4(1):49-60, 2003.

[240] T. M. Squires, R. J. Messinger, and S. R. Manalis. Making it stick: convection, reaction and diffusion in surface-based biosensors. Nature Biotechnology, 26(4):417-426, APR 2008.

[241] M. Stravalaci, M. Beeg, M. Salmona, and M. Gobbi. Use of surface plasmon resonance to study the elongation kinetics and the binding properties of the highly amyloidogenic a beta(1-42) peptide, synthesized by depsi-peptide technique. Biosensors \& Bioelectronics, 26(5):2772-2775, 2011.

[242] A. M. Streets and Y. Y. Huang. Chip in a lab: Microfluidics for next generation life science research. Biomicrofluidics, 7(1), 2013.

[243] J. C. Stroud, C. Liu, P. K. Teng, and D. Eisenberg. Toxic fibrillar oligomers of amyloid- $\beta$ have cross- $\beta$ structure. Proceedings of the National Academy of Sciences, 109:7717-7722, 2012.

[244] N. L. Stults, P. Lin, M. Hardy, Y. C. Lee, Y. Uchida, Y. Tsukada, and T. Sugimori. Immobilization of proteins on partially hydrolyzed agarose beads. $A n$ alytical Biochemistry, 135(2):392-400, 1983.

[245] M. Sunde, L. C. Serpell, M. Bartlam, P. E. Fraser, M. B. Pepys, and C. C. F. Blake. Common core structure of amyloid fibrils by synchrotron x-ray diffraction. Journal of Molecular Biology, 273(3):729-739, 1997.

[246] L. Surugiu, E. S. Dey, J. Svitel, S. Pirvutoiu, and B. Danielsson. Dextranmodified surface for highly sensitive chemiluminescent elisa. Analyst, 126(10):1633-1635, 2001. 
[247] G. Szabo, P. S. Pine, J. L. Weaver, M. Kasari, and A. Aszalos. Epitope mapping by photobleaching fluorescence resonance energy-transfer measurements using a laser scanning microscope system. Biophysical Journal, 61(3):661-670, 1992.

[248] E. J. Szili, S. Kumar, R. S. C. Smart, and N. H. Voelcker. Generation of a stable surface concentration of amino groups on silica coated onto titanium substrates by the plasma enhanced chemical vapour deposition method. Applied Surface Science, 255(15):6846-6850, 2009.

[249] V. Tougu, A. Karafin, K. Zovo, R. S. Chung, C. Howells, A. K. West, and P. Palumaa. Zn(ii)- and cu(ii)-induced non-fibrillar aggregates of amyloid-beta (1-42) peptide are transformed to amyloid fibrils, both spontaneously and under the influence of metal chelators. Journal of Neurochemistry, 110(6):17841795, 2009.

[250] E. J. M. Tournier, J. Wallach, and P. Blond. Sulfosuccinimidyl 4-(nmaleimidomethyl)-1-cyclohexane carboxylate as a bifunctional immobilization agent. optimization of the coupling conditions. Analytica Chimica Acta, 361(12):33-44, 1998.

[251] V. V. Tsukruk, I. Luzinov, and D. Julthongpiput. Sticky molecular surfaces: Epoxysilane self-assembled monolayers. Langmuir, 15(9):3029-3032, 1999.

[252] R. M. Vabulas, S. Raychaudhuri, M. Hayer-Hartl, and F. U. Hartl. Protein folding in the cytoplasm and the heat shock response. Cold Spring Harbor Perspectives in Biology, 2(12), 2010.

[253] M. K. Verma, A. Majumder, and A. Ghatak. Embedded template-assisted fabrication of complex microchannels in pdms and design of a microfluidic adhesive. Langmuir, 22(24):10291-5, 2006.

[254] E. Verpoorte and N. F. De Rooij. Microfluidics meets mems. Proceedings of the Ieee, 91(6):930-953, 2003.

[255] M. G. von Muhlen, N. D. Brault, S. M. Knudsen, S. Y. Jiang, and S. R. Manalis. Label-free biomarker sensing in undiluted serum with suspended microchannel resonators. Analytical Chemistry, 82(5):1905-1910, 2010.

[256] D. M. Walsh, D. M. Hartley, Y. Kusumoto, Y. Fezoui, M. M. Condron, A. Lomakin, G. B. Benedek, D. J. Selkoe, and D. B. Teplow. Amyloid beta-protein fibrillogenesis - structure and biological activity of protofibrillar intermediates. Journal of Biological Chemistry, 274(36):25945-25952, 1999. 
[257] D. Wang, S. Liu, B. J. Trummer, C. Deng, and A. Wang. Carbohydrate microarrays for the recognition of cross-reactive molecular markers of microbes and host cells. Nature Biotechnology, 20(3):275-81, 2002.

[258] Y. Wang, R. D. Lowe, Y. X. Mejia, H. Feindt, S. Steltenkamp, and T. P. Burg. A fluorescence based method for the quantification of surface functional groups in closed micro- and nanofluidic channels. Biomicrofluidics, 7(2), 2013.

[259] R. V. Ward, K. H. Jennings, R. Jepras, W. Neville, D. E. Owen, J. Hawkins, G. Christie, J. B. Davis, A. George, E. H. Karran, and D. R. Howlett. Fractionation and characterization of oligomeric, protofibrillar and fibrillar forms of beta-amyloid peptide. Biochemical Journal, 348:137-144, 2000.

[260] C. A. Waudby, T. P. J. Knowles, G. L. Devlin, J. N. Skepper, H. Ecroyd, J. A. Carver, M. E. Welland, J. Christodoulou, C. M. Dobson, and S. Meehan. The interaction of alpha b-crystallin with mature alpha-synuclein amyloid fibrils inhibits their elongation. Biophysical Journal, 98(5):843-851, 2010.

[261] G. T. Webster, J. Dusting, S. Balabani, and E. W. Blanch. Detecting the early onset of shear-induced fibril formation of insulin in situ. Journal of Physical Chemistry B, 115(11):2617-2626, 2011.

[262] D. B. Weibel, W. R. DiLuzio, and G. M. Whitesides. Microfabrication meets microbiology. Nature Reviews Microbiology, 5(3):209-218, 2007.

[263] Y. C. Weng, F. F. Delgado, S. Son, T. P. Burg, S. C. Wasserman, and S. R. Manalis. Mass sensors with mechanical traps for weighing single cells in different fluids. Lab on a Chip, 11(24):4174-4180, 2011.

[264] P. Westermark, M. D. Benson, J. N. Buxbaum, A. S. Cohen, B. Frangione, S. I. Ikeda, C. L. Masters, G. Merlini, M. J. Saraiva, and J. D. Sipe. Amyloid: Toward terminology clarification - report from the nomenclature committee of the international society of amyloidosis. Amyloid-Journal of Protein Folding Disorders, 12(1):1-4, 2005.

[265] D. A. White, A. K. Buell, C. M. Dobson, M. E. Welland, and T. P. J. Knowles. Biosensor-based label-free assays of amyloid growth. Febs Letters, 583(16):2587-2592, 2009.

[266] G. M. Whitesides. The origins and the future of microfluidics. Nature, 442(7101):368-373, 2006. 
[267] G. M. Whitesides, J. P. Mathias, and C. T. Seto. Molecular self-assembly and nanochemistry - a chemical strategy for the synthesis of nanostructures. Science, 254(5036):1312-1319, 1991.

[268] M. R. Wilson, J. J. Yerbury, and S. Poon. Potential roles of abundant extracellular chaperones in the control of amyloid formation and toxicity. Molecular Biosystems, 4(1):42-52, 2008.

[269] L. S. Wong, F. Khan, and J. Micklefield. Selective covalent protein immobilization: Strategies and applications. Chemical Reviews, 109(9):4025-4053, 2009 .

[270] J. Wu and M. Gu. Microfluidic sensing: state of the art fabrication and detection techniques. Journal of Biomedical Optics, 16(8), AUG 2011.

[271] Y. J. Xing and E. Borguet. Specificity and sensitivity of fluorescence labeling of surface species. Langmuir, 23(2):684-688, 2007.

[272] W. F. Xue, S. W. Homans, and S. E. Radford. Amyloid fibril length distribution quantified by atomic force microscopy single-particle image analysis. Protein Engineering Design \& Selection, 22(8):489-496, 2009.

[273] J. Yakovleva, R. Davidsson, A. Lobanova, M. Bengtsson, S. Eremin, T. Laurell, and J. Emneus. Microfluidic enzyme immunoassay using silicon microchip with immobilized antibodies and chemiluminescence detection. Anal Chem, 74(13):2994-3004, 2002.

[274] L. Yu, C. M. Li, Y. S. Liu, J. Gao, W. Wang, and Y. Gan. Flow-through functionalized pdms microfluidic channels with dextran derivative for elisas. Lab on a Chip, 9(9):1243-1247, 2009.

[275] L. Yu, C.M. Li, Q. Zhou, and J.H.T. Luong. Poly(vinyl alcohol) functionalized poly(dimethylsiloxane) solid surface for immunoassay. Bioconjugate Chemistry, 18(2):281-284, 2007.

[276] E. Zerovnik. Amyloid-fibril formation - proposed mechanisms and relevance to conformational disease. European Journal of Biochemistry, 269(14):3362-3371, 2002 .

[277] X. W. Zhuang, T. Ha, H. D. Kim, T. Centner, S. Labeit, and S. Chu. Fluorescence quenching: A tool for single-molecule protein-folding study. Proceedings of the National Academy of Sciences of the United States of America, 97(26):14241-14244, 2000. 
[278] C. Ziegler. Cantilever-based biosensors. Analytical and Bioanalytical Chemistry, 379(7-8):946-959, 2004.

[279] K. Zovo, E. Helk, A. Karafin, V. Tougu, and P. Palumaa. Label-free highthroughput screening assay for inhibitors of alzheimer's amyloid-beta peptide aggregation based on maldi ms. Analytical Chemistry, 82(20):8558-8565, 2010. 
Appendix 
Table 6.1: Fitting parameters for time-dependent fluorescence detection ${ }^{[1]}$.

\begin{tabular}{cccccccccc}
\hline \hline Parameter & $1.25 \mu \mathrm{M}$ & $2.5 \mu \mathrm{M}$ & $3.5 \mu \mathrm{M}$ & $5 \mu \mathrm{M}$ & $10 \mu \mathrm{M}$ & $20 \mu \mathrm{M}$ & $40 \mu \mathrm{M}$ & $5 \mu \mathrm{M}$ pure & $10 \mu \mathrm{M}$ pure \\
\hline$A_{1}$ & 2200 & 3130 & 7187 & 12194 & 18010 & 24142 & 33971 & 16099 & 16949 \\
$\tau_{p h 1}$ & 4272 & 7413 & 7472 & 5147 & 4937 & 7451 & 6735 & 8733 & 8677 \\
$A_{2}$ & 403 & 1378 & 1831 & 0 & 0 & 0 & 0 & 0 & 0 \\
$\tau_{p h 2}$ & 247 & 704 & 802 & $\mathrm{NA}$ & $\mathrm{NA}$ & $\mathrm{NA}$ & $\mathrm{NA}$ & $\mathrm{NA}$ & $\mathrm{NA}$ \\
$A_{3}$ & 0 & 0 & 0 & 0.031 & 0.105 & 0.251 & 0.344 & 0.565 & 0.584 \\
$\tau_{\text {dequench }}$ & $\mathrm{NA}$ & $\mathrm{NA}$ & $\mathrm{NA}$ & 34 & 106 & 149 & 213 & 182 & 133 \\
Adj. R-square & 0.9979 & 0.9986 & 0.9969 & 0.9850 & 0.9867 & 0.9946 & 0.9916 & 0.9881 & 0.9722 \\
\hline \hline
\end{tabular}


Table 6.2: Standard statistical errors for fitting parameters ${ }^{[1]}$.

\begin{tabular}{cccccccccc}
\hline \hline Error & $1.25 \mu \mathrm{M}$ & $2.5 \mu \mathrm{M}$ & $3.5 \mu \mathrm{M}$ & $5 \mu \mathrm{M}$ & $10 \mu \mathrm{M}$ & $20 \mu \mathrm{M}$ & $40 \mu \mathrm{M}$ & $5 \mu \mathrm{M}$ pure & $10 \mu \mathrm{M}$ pure \\
\hline$A_{1}$ & 16 & 78 & 277 & 68 & 134 & 74 & 152 & 102 & 127 \\
$\tau_{p h 1}$ & 60 & 415 & 608 & 87 & 95 & 81 & 93 & 181 & 285 \\
$A_{2}$ & 17 & 73 & 264 & 0 & 0 & 0 & 0 & 0 & 0 \\
$\tau_{p h 2}$ & 23 & 48 & 130 & $\mathrm{NA}$ & $\mathrm{NA}$ & $\mathrm{NA}$ & $\mathrm{NA}$ & $\mathrm{NA}$ & $\mathrm{NA}$ \\
$A_{3}$ & 0 & 0 & 0 & 0.019 & 0.013 & 0.005 & 0.005 & 0.013 & 0.012 \\
$\tau_{\text {dequench }}$ & $\mathrm{NA}$ & $\mathrm{NA}$ & $\mathrm{NA}$ & 40 & 28 & 6 & 8 & 9 & 6 \\
\hline \hline
\end{tabular}




\section{Publications}

\section{Journals}

- Yu Wang, Rachel D. Lowe, Yara X. Mejia, Holger Feindt, Siegfried Steltenkamp and Thomas P. Burg, A Fluorescence Based Method For the Quantification of Surface Functional Groups in Closed Micro- and Nanofluidic Channels, Biomicrofluidics, 7, 026513, (2013).

- Mario M. Modena, Yu Wang, Dietmar Riedel and Thomas P. Burg, Resolution Enhancement of Suspended Microchannel Resonators for Weighing of Biomolecular Complexes in Solution, Lab on a Chip, 14, 342-350, (2014).

\section{Conference}

- Yu Wang, Mario M. Modena and Thomas P. Burg, Label-Free Characterization of Amyloid Growth by Suspended Microchannel Resonators, Proceedings of 17th International Conference on Miniaturized Systems for Chemistry and Life Sciences, 1508-1510, (2013).

- Mario M. Modena, Yu Wang and Thomas P. Burg, Resolution Enhancement of Suspended Microchannel Resonators by Mass Correlation Analysis, Proceedings of 17 th International Conference on Miniaturized Systems for Chemistry and Life Sciences, 188-190, (2013). 